Florida International University FIU Digital Commons

6-22-2018

\title{
Aptamer Sensors for Drugs of Abuse and Medical Biomarkers: Design, Engineering and Application in Complex Samples
}

Daniel Roncancio

Florida International University, dronc001@fiu.edu

DOI: 10.25148 /etd.FIDC006889

Follow this and additional works at: https:// digitalcommons.fiu.edu/etd

Part of the Chemistry Commons

\section{Recommended Citation}

Roncancio, Daniel, "Aptamer Sensors for Drugs of Abuse and Medical Biomarkers: Design, Engineering and Application in Complex Samples" (2018). FIU Electronic Theses and Dissertations. 3826.

https://digitalcommons.fiu.edu/etd/3826 


\title{
FLORIDA INTERNATIONAL UNIVERSITY
}

\author{
Miami, Florida
}

\section{APTAMER SENSORS FOR DRUGS OF ABUSE AND MEDICAL BIOMARKERS: DESIGN, ENGINEERING, AND APPLICATION IN COMPLEX SAMPLES}

\author{
A dissertation submitted in partial fulfillment of \\ the requirements for the degree of \\ DOCTOR OF PHILOSOPHY \\ in \\ CHEMISTRY \\ by \\ Daniel Roncancio
}

2018 
To: Dean Michael R. Heithaus

College of Arts, Sciences and Education

This dissertation, written by Daniel Roncancio, and entitled Aptamer Sensors for Drugs of Abuse and Medical Biomarkers: Design, Engineering, and Application in Complex Samples, having been approved in respect to style and intellectual content, is referred to you for judgment.

We have read this dissertation and recommend that it be approved.

Alexander Mebel

Anthony DeCaprio

Anthony McGoron

Kevin O'Shea

Yi Xiao, Major Professor

Date of Defense: June 22, 2018

The dissertation of Daniel Roncancio is approved.

Dean Michael R. Heithaus

College of Arts, Sciences and Education

Andrés G. Gil

Vice President for Research and Economic Development and Dean of the University Graduate School

Florida International University, 2018 
(C) Copyright 2018 by Daniel Roncancio

All rights reserved. 


\section{DEDICATION}

I dedicate this work to my family. Your encouragement has empowered me to achieve beyond my wildest expectations. Thank you for all the effort, sacrifice and love. 


\section{ACKNOWLEDGMENTS}

This work was completed with the help of a tremendous group of individuals. I would like to thank Dr. Yi Xiao for her unrelenting mentoring, patience and support. While there were difficult moments in this process, Dr. Xiao sacrificed many hours to make it easier and simpler. Dr. Alexander Mebel, thank you for your support and encouragement during my time at FIU. You are not only a great teacher but also a great mentor. Dr. Anthony DeCaprio, thank you for your guidance, support and trust. You facilitated our work tremendously. Dr. Kevin O'Shea, thank you for your words of advice. Your words of planning have improved my efficiency and allowed me to complete this work in time. Dr. Anthony McGoron, thank you for your detailed analysis, attentiveness and questions.

I would also like to acknowledge the financial and professional help from the McNair graduate fellowship and the dissertation year fellowship. Their support allowed me incredible scheduling freedom to research and publish high impact manuscripts. 


\title{
ABSTRACT OF THE DISSERTATION
}

\section{APTAMER SENSORS FOR DRUGS OF ABUSE AND MEDICAL BIOMARKERS: DESIGN, ENGINEERING, AND APPLICATION IN COMPLEX SAMPLES}

\author{
by \\ Daniel Roncancio \\ Florida International University, 2018 \\ Miami, Florida \\ Professor Yi Xiao, Major Professor
}

Aptamers are short oligonucleotide sequences (DNA or RNA) capable of high affinity and specific binding to a molecule or a family of molecules. Aptamers are lower in cost and exhibit higher reproducibility when compared to antibodies and thus are well-suited for recognition and detection of small molecular targets such as drugs of abuse and small medical biomarkers. While aptamers have been extensively utilized for development of small molecule sensors, several limitations prevent measurements of complex or real-world samples. This dissertation describes methods, technologies, and assays that were developed with the goal of producing and/or improving aptamer-based sensors for target detection in complex samples. Aptamer engineering is detailed as an important facet of maximizing aptamer-sensor sensitivity and specificity, along with adaptation to various read-out mechanisms for improved selectivity. In chapter 3 , an aptamer 
sensor for cocaine is developed based on binding between the fluorophore ATMND to the cocaine aptamer which results in quenching (i.e., 'turn-off') of the fluorescence of ATMND. Cocaine binding results in displacement of the ATMND and recovery of the fluorescence signal. Detection of cocaine is demonstrated with an engineered cocaine aptamer with higher affinity for cocaine, permitting over a 50 -fold increase in sensitivity over other aptamer-based sensors. The method can be used in dilute biological fluids (e.g., saliva) with a single step reaction (seconds) and robust signal output. In chapter 4 , a new adenosine specific aptamer is identified through rational engineering of a previously reported ATP-binding aptamer. The new adenosine aptamer is utilized to develop an electrochemical sensor for detection of adenosine in undiluted serum. The method displays 40 -fold higher sensitivity in undiluted serum measurements over previously reported aptamer-based sensors for adenosine but also demonstrates specificity for adenosine over ATP, ADP and AMP that has not been previously reported. In chapter 5 , a nuclease-guided truncation method is developed to yield optimal structure-switching aptamer sequences for the emergent illicit drug methylenedioxypyrovalerone (MDPV) and medical biomarkers ATP and deoxycorticosterone 21-glucoside (DOG). The method intelligently removes unessential nucleotides, producing truncated aptamer sequences with structureswitching functionality. This technique will be immediately useful for simple and low-cost development of aptamer-based electrochemical sensors. 


\section{TABLE OF CONTENTS}

CHAPTER

PAGE

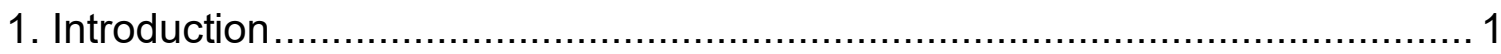

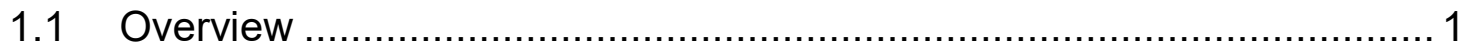

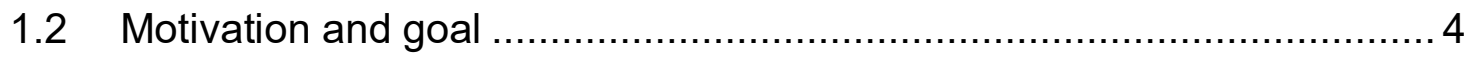

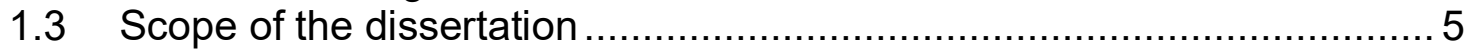

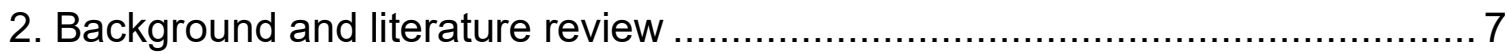

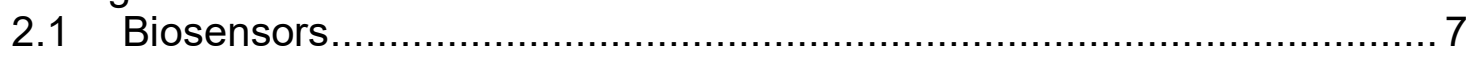

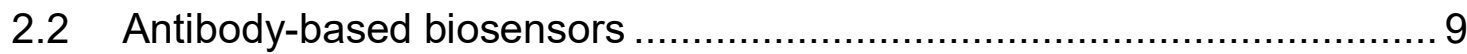

2.2.1 Competitive enzyme-linked immunosorbent assay (ELISA).............9

2.2.2 Homogenous enzyme-linked immunoassays .............................. 10

2.2.3 Kinetic interaction of microparticles in solution (KIMS) .................. 11

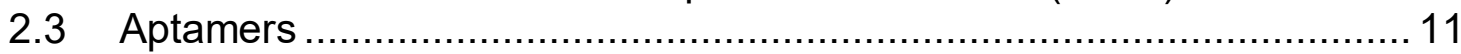

2.3.1 Discovery of aptamers and SELEX …................................... 12

2.3.2 Systematic evolution by exponential enrichment (SELEX) .............. 12

2.3.3 Aptamer-based sensors for small molecules ............................... 15

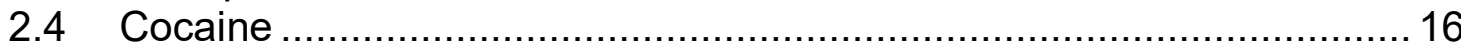

2.4.1 Cocaine metabolite detection with antibodies ................................ 17

2.4.2 Cocaine detection with aptamers ............................................ 18

2.4.3 (2)-amino-5,6,7-trimethyl-1,8-naphthyridine (ATMND) ................... 20

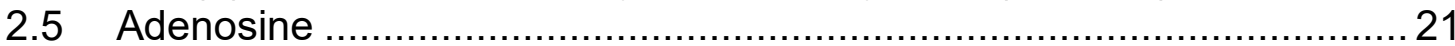

2.5.1 Adenosine concentration measurements ....................................22

2.5.2 Aptamer-based adenosine measurements ................................ 23

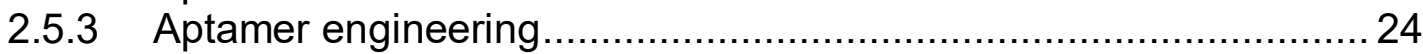

2.5.4 Electrochemical aptamer-based (E-AB) sensors .........................2 24

$2.6 \quad$ Aptamer structure-switching …................................................... 26

2.6.1 Nuclease-guided truncation of aptamers.................................. 27

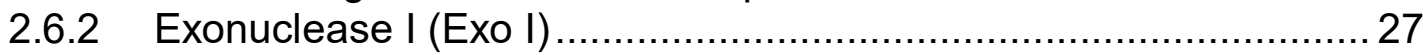

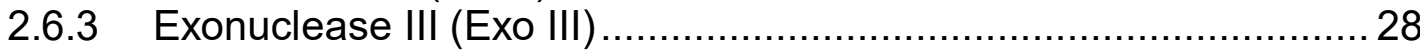

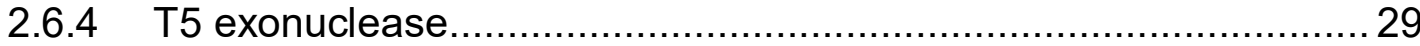

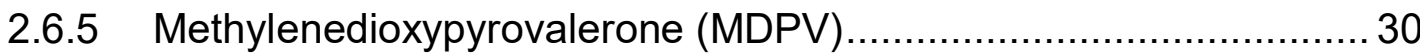

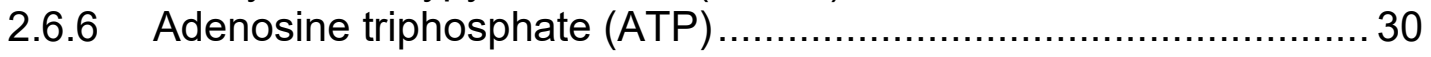

3. A Label-Free Aptamer-Fluorophore Assembly for Rapid and Specific

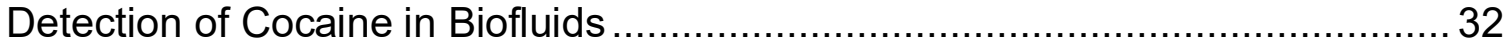

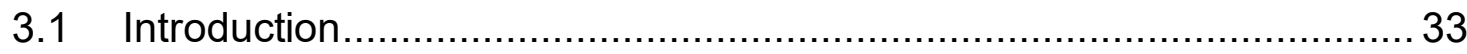

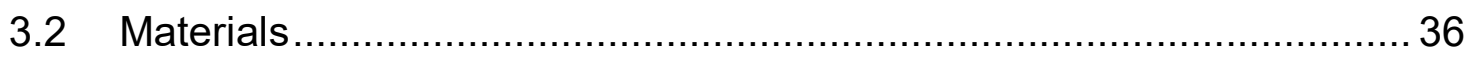

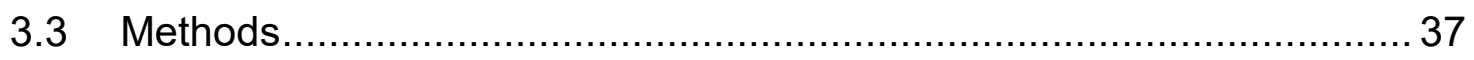

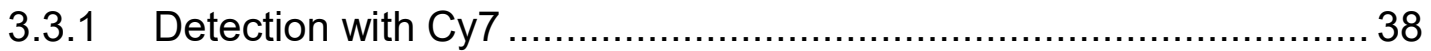

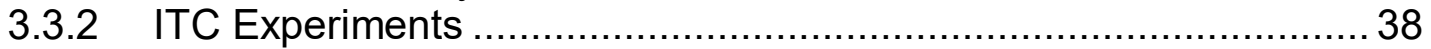

3.3.3 Detection of Cocaine in Urine, Serum, and Saliva ........................ 38 


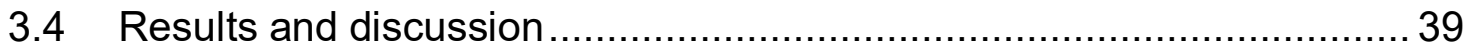

3.4.1 Modeling data for binding of ATMND to 38-GC ........................... 48

3.4.2 Mutation of binding pocket nucleotides ...................................... 52

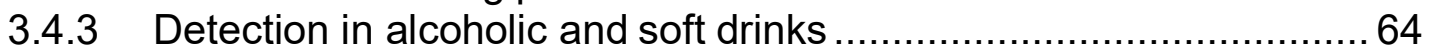

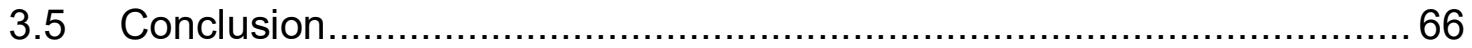

4. Engineering an adenosine-specific aptamer and electrochemical detection

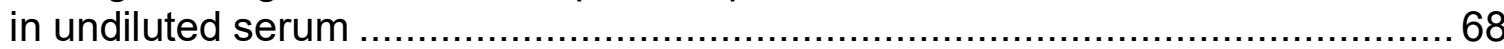

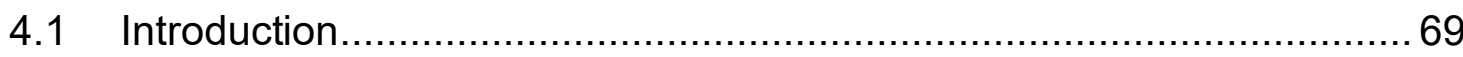

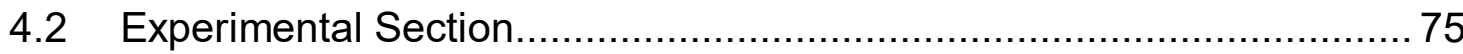

4.2.1 Polyacrylamide gel electrophoresis (PAGE) ............................ 76

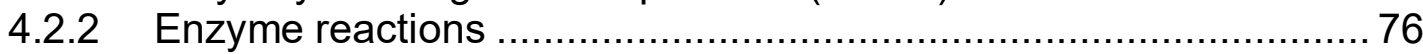

4.2.3 Isothermal Titration Calorimetry (ITC) …................................ 77

4.2.4 Circular Dichroism (CD) .................................................... 78

4.2.5 Aptamer immobilization to gold electrodes.................................. 78

4.2.6 Electrochemical measurements .............................................. 79

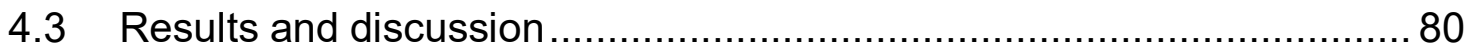

4.3.1 Cross-reactivity of the original ATP-binding aptamer .................... 80

4.3.2 Engineering of an adenosine-specific aptamer ............................. 83

4.3.3 Addition of Exonuclease I to remove background fragments ...........85

4.3.4 Implications of magnesium concentration on Ade-33 specificity...... 87

4.3.5 Probing structure-switching functionality with exonuclease I...........91

4.3.6 Structure-switching characterization with circular dichroism ........... 94

4.3.7 Characterization of aptamer-target binding with ITC ......................96

4.3.8 Design of methylene-blue modified aptamer sequence.................. 100

4.3.9 Electrode surface modification and effects on signaling ............... 102

4.3.10 Detection of adenosine in undiluted and unprocessed serum ........ 105

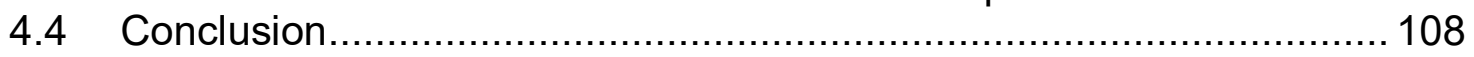

5. Nuclease-guided truncation of aptamers for optimal structure-switching functionality and application in electrochemical sensors ................................. 110

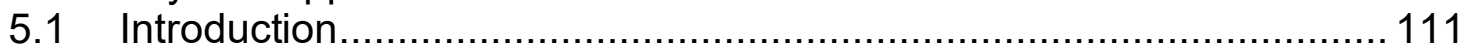

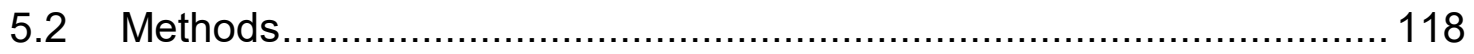

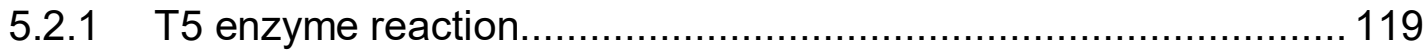

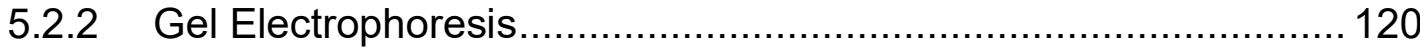

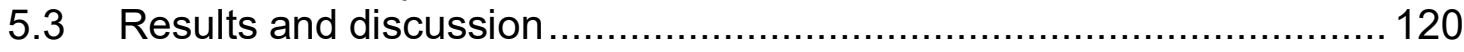

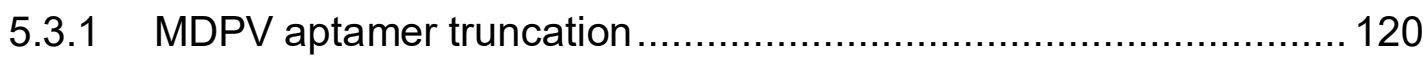

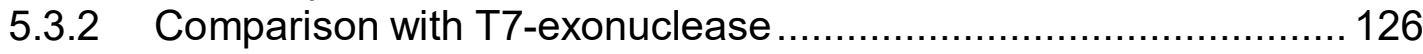

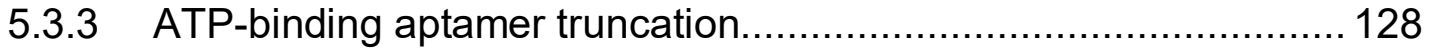

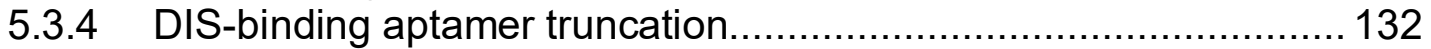

5.3.5 T5 activity on DOG-binding aptamer ...................................... 135

5.3.6 Engineering a DOG-binding aptamer for T5 truncation ................. 138

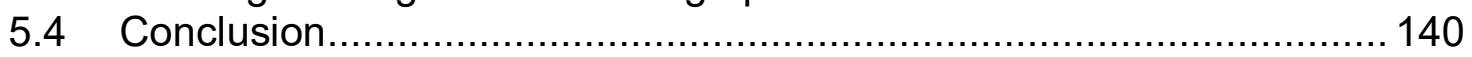

6. Summary and future directions ......................................................... 141 


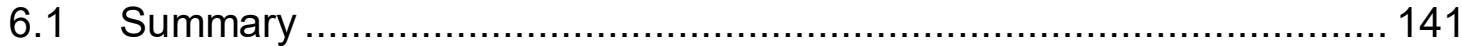

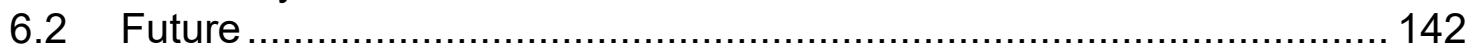

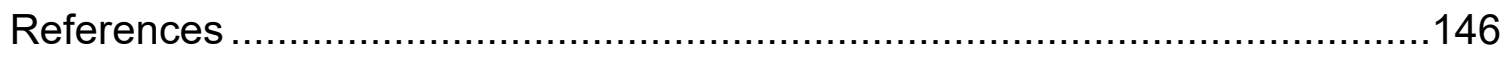

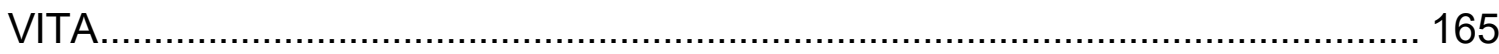




\section{LIST OF FIGURES}

FIGURE

PAGE

1. Example of a biosensor for human chorionic gonadotropin (hCG) and its

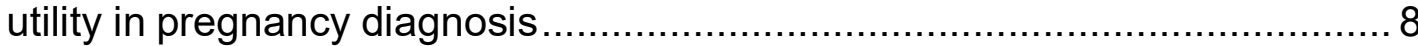

2. Example schematic of target immobilized SELEX …............................... 15

3. Structures of the MNS-4.1, 38-GT, and 38-GC aptamers .......................... 35

4. Our aptamer-based sensor rapidly responds in the presence of cocaine. .... 41

5. Stem-length effects on fluorophore binding and displacement by cocaine $\ldots . .43$

6. ITC data demonstrated that MNS-4.1 and 38-GT binds ATMND ................ 46

7. 38-GC binds both cocaine and ATMND.............................................. 47

8. Effect of different concentrations of ATMND on fluorescence signal gain..... 48

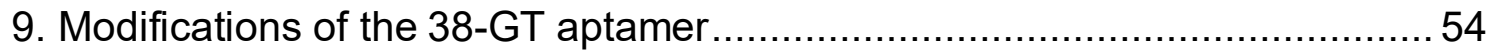

10. 38-GC-M1 and 38-GC-M2 demonstrated a very weak binding affinity for

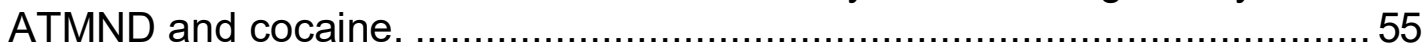

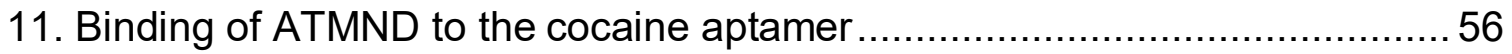

12. Sensitivity and specificity of the ATMND-based sensor in the reaction buffer

13. Calibration curve for a 38-GC-Cy7-based sensor ................................... 58

14. Cross-reactivity of the ATMND-sensor for several compounds of interest in cocaine measurements

15. Successful detection of cocaine spiked into different dilutions of urine, saliva and serum with 38-GCATMND.

16. $5 \%$ urine and serum samples emit fluorescence within the wavelength range from $375 \mathrm{~nm}$ to $600 \mathrm{~nm}$ when excited at $358 \mathrm{~nm}$.

17. Fluorescence intensities for different concentrations of various biofluids 62 
18. Calibration curves for ATMND-38-GC-displaced cocaine detection in biological samples

19. Fluorescence intensities of ATMND in different concentrations of saliva and serum

20. Calibration curves for cocaine detection with ATMND-38-GC in $2.5 \%$ serum

21. Detection of cocaine in spiked soft drinks 65

22. Detection of cocaine in alcoholic drinks 65

23. Specific target binding inhibited exonuclease III digestion of the aptamer 4-nt away from the presumed binding site.

24. Exonuclease III screening test of engineered sequences and identification of an adenosine-specific aptamer termed Ade-33

25. Addition of exonuclease I to remove single-stranded DNA fragments leftover from exonuclease III reaction.

26. Exonuclease III can identify multiple aptamer fragments with apparent structure-switching functionality.

27. Magnesium effect in the binding specificity of Ade-33 and ATP-33 aptamers

28. Effect of sodium on target binding.

29. Cross-reactivity testing of a new binding sequence that is specific for adenosine

30. CD spectra for Ade-29 and Ade-30 without addition of target 95

31. Test of conformational change of Ade-29, Ade-30 and Ade-30m with Circular Dichroism (CD).

32. Proposed mechanism of Ade-30 specificity for Adenosine due to steric hinderance with ATP and ADP at low magnesium concentration. 98

33. ITC binding experiments at $10 \mathrm{mM}$ magnesium for the Ade-30 sequence.... 99

34. Binding affinity of Ade-29 to adenosine, ADP and ATP under optimal binding conditions (10 mM BTP, $1 \mathrm{mM}$ magnesium at $\mathrm{pH} 8.5$ ) 
35. Design of methylene blue modified adenosine aptamer sequence:

Ade-29-MB

36. Platform for electrochemical sensing of adenosine using the adenosine specific Ade-29-MB aptamer

37. Magnesium and sodium effect on electrochemical detection. 103

38. MCH and DTT effect on electrochemical sensor and optimization. 105

39. Electrochemical detection of adenosine in buffer and serum 107

40. Serum optimization and cross-reactivity in undiluted serum 107

41. Adenosine specific detection and cross-reactivity with ATP, ADP, AMP and GTP

42. T5-guided truncation of an MDPV-binding aptamer yielding a structureswitching sequence.

43. Time course of $T 5$ reaction and characterization of enzyme reaction products visualized utilizing gel electrophoresis

44. T5 inhibition is caused by formation of a MDPV-aptamer complex and not by MDPV itself

45. Comparison of aptamer truncation with T5-and T7-exonuclease.

46. Time course of T5 reaction with ATP-binding aptamer 130

47. T5 inhibition is caused by formation of aptamer-ATP complexes and not by ATP directly

48. T5-guided truncation of DIS-binding aptamer to identify structure-switching fragments

49. Test of T5 inhibition by the steroid molecule DIS

50. Digestion rate of T5 on 5' overhangs and double-stranded DNA

51. Time course of T5 digestion of DOG.-3 aptamer structure and enzymatic products visualized using gel electrophoresis

52. T5-guided truncation of redesigned DOG aptamer sequence. 139 


\section{LIST OF ABBREVIATIONS}

A

ADA

Ade

ADK

ADP

AMP

APS

ATMND

ATP

BSA

C

CD

CE

Cy7

DI

DIS adenine

adenosine deaminase

adenosine

adenosine kinase

adenosine diphosphate

adenosine monophosphate

ammonium persulfate

2-amino-5,6,7-trimethyl-1,8-naphthyridine

adenosine triphosphate

bovine serum albumin

cytosine

circular dichroism

capillary electrophoresis

3,3'-diethylthiatricarbocyanine iodide

deionized water

dehydroisoandrosterone 3-sulfate 


$\begin{array}{ll}\text { DMSO } & \text { dimethyl sulfoxide } \\ \text { DOG } & \text { deoxycorticosterone 21-glucoside } \\ \text { DTT } & \text { dithiothreitol } \\ \text { E-AB } & \text { electrochemical aptamer-based } \\ \text { ELISA } & \text { enzyme-linked immunosorbent assay } \\ \text { EMIT } & \text { enzyme-linked immunoassays } \\ \text { Exo I } & \text { exonuclease I } \\ \text { Exo III } & \text { exonuclease III } \\ \text { G } & \text { guanine } \\ \text { KTP } & \text { guanosine triphosphate } \\ \text { Kd } & \text { isothermal titration calorimetry } \\ \text { hCG } & \text { human chorionic gonadotropin } \\ \text { HPLC } & \text { high performance liquid chromatography } \\ \text { KS } & \end{array}$




$\begin{array}{ll}\text { LOD } & \text { limit of detection } \\ \text { MB } & \text { methylene-blue } \\ \text { MCH } & \text { 6-mercapto-1-hexanol } \\ \text { MDPV } & \text { methylenedioxypyrovalerone } \\ \text { nt } & \text { nucleotides } \\ \text { PAGE } & \text { polyacrylamide gel electrophoresis } \\ \text { PBS } & \text { phosphate buffered saline } \\ \text { PCR } & \text { polymerase chain reaction } \\ \text { RSD } & \text { relative standard deviation } \\ \text { SELEX } & \text { tris } \\ \text { TBE } & \text { thetramethylethylenediamine } \\ \text { SNP } & \text { single nucleotide polymorphism } \\ \text { SWV } & \text { square wave voltammetry } \\ \text { TCEP } & \text { thym }\end{array}$




\section{CHAPTER 1}

\section{Introduction}

\subsection{Overview}

The detection of small molecules is integral to various aspects of modern living including medical diagnostics, environmental monitoring, and law enforcement. Accurate identification of a single molecule in a heterogeneous mixture requires high specificity to discriminate between competing molecules and high sensitivity to correctly measure the mean concentration of even trace analyte amounts. Instrumental analysis provides the highest discriminatory level (i.e., high specificity) and high sensitivity, thus it is generally accepted to be the gold standard of chemical analysis. However, these methods still require expensive and cumbersome machines, expert technicians, complex procedures, and long turnaround times for sample analysis. Biosensors are devices which combine highly specific and high-affinity molecular recognition of an analyte with simple and lowcost signal transduction. Biosensors have demonstrated similar analytical capabilities as expensive and complex analytical instruments but with exceptional convenience, instantaneous results, and lower cost. Today, a person can walk to a drugstore and purchase bio-sensing devices, aimed at detecting vastly different molecules including exogenous compounds such as drugs of abuse and endogenous compounds like medical biomarkers. Within minutes, an accurate representation of the analyte can be obtained in complex biological fluids without requiring any specialized training. The analyte concentration can be used to diagnose disease, monitor health status or drug use. 
Biosensors are designed by combination of a recognition element and a transduction element. The recognition element provides specific binding and highaffinity interactions with the analyte against other molecules present in a sample. The transduction element converts the binding interaction between the recognition element and the analyte into a measurable signal that can be interpreted visually or instrumentally. Recognition elements include biological molecules such as antibodies, enzymes and more recently, aptamers (i.e., DNA/RNA oligonucleotides). Antibody-based biorecognition is still widespread because of the excellent analytical characteristics of antibodies (high specificity and sensitivity) and nearly sixty years of research and development. ${ }^{1}$ More than two million antibodies are commercially available from hundreds of companies. ${ }^{2}$ Several examples of antibody-based sensors are commonly known to the general population including: pregnancy tests, ovulation kits, and drug screening immunoassays. Unfortunately, the generation of antibodies requires animal immunization which is not universally applicable for every molecule, such as in the case of immunosuppressive molecules and toxins. ${ }^{3,4}$ Additionally, antibody reliability and specificity is highly source-dependent with susceptibility to batch-tobatch variation and non-specific binding to off-target molecules. Indeed, landmark publications have been retracted because of difficulty in reproducing scientific findings obtained with antibodies. Authors of such publications have advised caution and recommended careful validation of individual antibody assays. ${ }^{5,6}$ In the context of small molecule detection, antibody-based sensors require specialized molecular components to transduce the binding interaction with the 
analyte into a measurable signal. For example, a chemically labeled target can be added to the sample as a signal marker by competing with the free target for the antibody binding site. ${ }^{7}$ Only the labeled-target can report interactions with the antibody and any disturbance of the interaction is a representative signal that free target is present. Similarly, an enzyme-labeled target can be utilized to compete with the free target. The enzyme-labeled target is inactivated when bound to the antibody but can regain its activity upon displacement from the antibody by free target in the sample. The active enzyme-labeled target can transform an exogenous substrate to generate a signal dependent on free target concentration. Thus, in the context of small molecule sensors, antibodies still do not offer direct transduction (i.e., signal generation) of analyte-antibody interactions.

Aptamers are single-stranded oligonucleotide-based bio-affinity reagents which mimic the specific recognition ability of antibodies but are chemically generated and synthesized, overcoming the necessity for animal immunization while providing unmatched reproducibility. ${ }^{8}$ Aptamers have been continuously improved since the first generation back in 1990, with recent examples demonstrating analytical performance surpassing antibodies. ${ }^{9}$ Aptamers are generated through an artificial evolutionary process that screens $>10^{13}$ unique sequences and only evolves sequences that provide high specificity and high affinity for the target of interest. The screening process can be customized to provide either exceptional specificity or cross-reactivity for a family of molecules. For example, counterselection targets can be added to the process to remove sequences with affinity for molecules with unwanted binding. Evolved aptamers can be accurately 
sequenced and chemically synthesized with incredible reproducibility and purity. Thus, aptamers always display the same inherent specificity and affinity regardless of the source unlike antibodies. However, it should be noted that aptamer-target interactions are dependent on experimental factors such as buffer conditions and temperature, thus reproducibility also depends on the experimenter. Aptamers are especially useful for detection of small molecule analytes with several methodologies not requiring addition of exogenous reagents or multiple assay steps. For example, Plaxco et al. described an aptamer-sensor that could be implanted in ambulant animals to monitor small molecule drugs in real time. ${ }^{10}$ Aptamers can be engineered and chemically modified with functional groups for signal transduction of the aptamer-analyte interaction, including analytical signal via a change in $\operatorname{color}^{11,12}$, fluorescence ${ }^{13,14}$, or electrochemical current ${ }^{15,16}$. Accordingly, aptamers have been utilized to develop biosensors for small molecules including exogenous drugs of abuse, endogenous medical biomarkers, and environmental toxins and pollutants. ${ }^{17}$

\subsection{Motivation and goal}

While the advantages of aptamer technology are well established, aptamer-based sensors have not reached commercial availability because of issues including limited sensitivity, inconsistent specificity (i.e., cross-reactivity) and susceptibility to matrix interferents (i.e., selectivity) ${ }^{8}$ The goal of this dissertation is to understand the physical characteristics of aptamer-target interactions and apply aptamer engineering to increase aptamer-sensor sensitivity, specificity (i.e., crossreactivity) and selectivity (i.e., specificity against matrix interferents), resulting in 
small molecule sensors that are functional in complex matrices. I describe methods of aptamer engineering and innovations in signal transduction that display improved analytical characteristics with specific examples including a cocaine sensor that improved the detection limit of aptamer-based sensors by 50 -fold, an

adenosine sensor that improved specificity against ATP, ADP and AMP and simultaneously demonstrated a 40 -fold improvement in sensitivity and a method to generalize the aptamer engineering process for small molecule detection in highly complex samples like serum.

\subsection{Scope of the dissertation}

To demonstrate the utility of aptamer-sensors for small molecule detection, I present three projects that focus on rational aptamer engineering to improve the sensitivity and specificity of current aptamers and outline a method to easily generate aptamer-based sensors that can be utilized in complex samples such as undiluted serum. Aptamer-based sensing of cocaine was previously limited because of insufficient sensitivity. In chapter 3, I describe a simple fluorescence aptamer-based sensor that I developed to detect cocaine in biological fluids and other complex samples such as alcoholic and soft drinks, exhibiting detection limits 50-fold higher than other aptamer-based sensors, yet displaying near instantaneous results, utilizing low cost reagents and microliter volumes. In chapter 4, I demonstrate the ability to engineer currently available aptamers to modulate their specificity. The previously reported adenosine aptamer is cross-reactive for multiple adenosine derivatives including adenosine, adenosine monophosphate, adenosine diphosphate and adenosine triphosphate, molecules which are all 
simultaneously present in biological fluids. The inherent cross-reactivity prevents utilization of this aptamer for specific measurements of adenosine, a well-known disease biomarker. An adenosine sensor is demonstrated that can instantaneously detect adenosine in undiluted serum, providing a tool for disease diagnostics and pathophysiological research. The sensor exhibits 40 -fold higher sensitivity compared to previously reported aptamer-based sensors with specificity against ATP, ADP and AMP that has not been demonstrated elsewhere. Conventional aptamer structures lack the structure-switching functionality required for development of sensors applicable in complex samples. In chapter 5 , I develop a nuclease-guided system to intelligently truncate aptamer sequences yielding aptamers which demonstrate optimal structure-switching functionality. The produced aptamers can be easily incorporated to sensors functional in undiluted complex samples. Specific examples include truncation of an methylenedioxypyrovalerone (MDPV)-binding aptamer, multiple steroid-binding aptamers, and an adenosine triphosphate (ATP)-binding aptamer. In combination, the body of work demonstrates the utility of aptamers for small molecule sensing, the ability and tools to engineer aptamers to maximize sensitivity and specificity and the adaptability of aptamers to methodologies that can be readily employed in highly complex samples such as biological fluids. 


\section{CHAPTER 2}

\section{Background and literature review}

\subsection{Biosensors}

Biosensors are devices utilized to measure the presence or concentration of a specific molecule in a sample solution. Biosensors are characterized by two main components: the recognition and transducing elements. ${ }^{18-20}$ The recognition element is the biomolecule component that provides discriminatory power for a single molecule and a high affinity interaction that permits measurements of even trace amounts of analyte. Recognition elements include enzymes, antibodies and more recently, aptamers (i.e., DNA/RNA molecules). For example, pregnancy tests utilize antibodies specific for human chorionic gonadotropin (hCG) to diagnose pregnancy at home. Levels of hCG hormone rise rapidly and consistently after implantation with concentrations peaking at 10,000 -fold above basal levels. ${ }^{21-23}$ While recognition elements provide extensive discriminatory power and strong interactions with a given molecule, they seldom transduce such an interaction into a measurable signal. The transducing element can convert the interaction between the recognition element and the analyte into a measurable signal. In the context of the pregnancy test, multiple antibodies work in tandem to transduce binding of hCG. The first antibody is immobilized on a test line and the second antibody is labeled with gold nanoparticles which provide a color signal (fig. 1a). In the presence of hCG, the two antibodies meet at the test line to form a sandwich around the hCG molecule as they bind different epitopes of the molecule and a colored line is observed on the test line from the gold nanoparticle-labeled antibody 
(fig. 1c and d, positive). In the absence of hCG, the line is not formed as the two antibodies do not bind each other (fig. $1 \mathrm{~d}$, negative). ${ }^{24,25} \mathrm{~A}$ control line is also included to signal the presence of the unbound antibody (fig. 1d). The recognition element and the transducer determine the analytical performance of a biosensor. The recognition element directly determines the specificity of the sensor while the sensitivity is a function of both the recognition element but is also strongly influenced by the transduction element. Biosensors are often characterized by the type of recognition element that is employed.
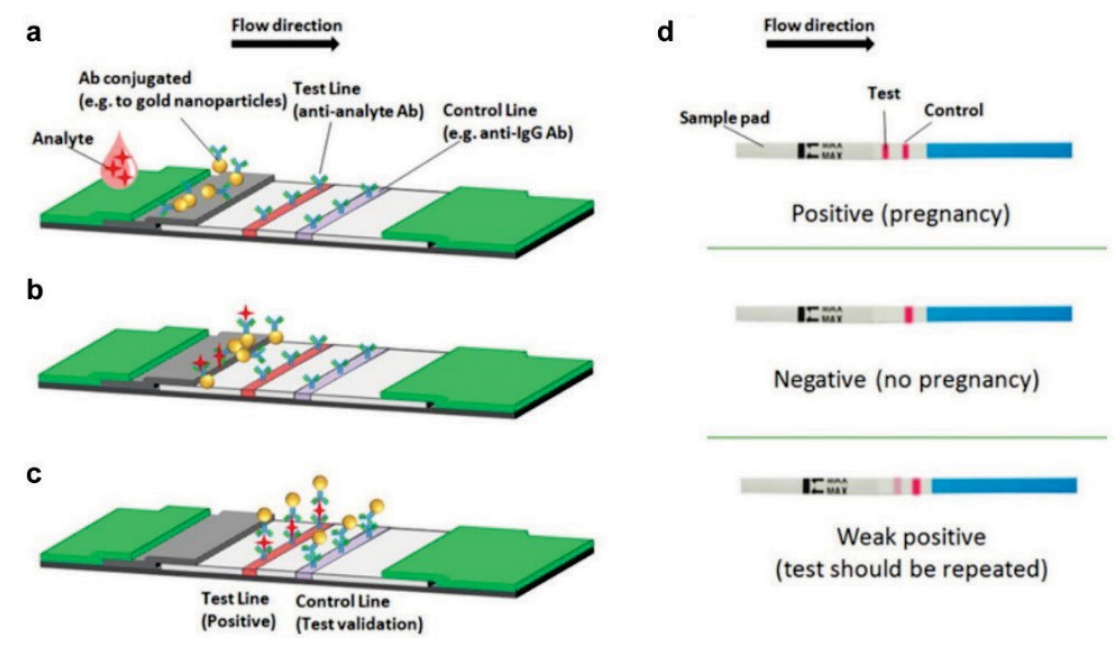

Figure 1. Example of a biosensor for human chorionic gonadotropin (hCG) and its utility in pregnancy diagnosis.

(a) Two antibodies specific for hCG are utilized. One antibody is immobilized on the test line and a second antibody is covalently labeled with gold nanoparticles for signal transduction. (b) Movement of the analyte and the labeled-antibody bring the analyte near the immobilized antibody. (c) If hCG is present in the sample, it will bind to the immobilized antibody and attract the secondary labeled antibody, forming a sandwich complex with a color line appearing on the test line. In the absence of hCG, the labeled antibody does not bind to the test line thus no color is observed. A control line is also utilized which binds the labeled antibodies. (d) Example results with a positive (two lines), negative (one line) and an intermediate sample that might be difficult to interpret. (Adapted from Koczula et al.). ${ }^{25}$ 


\subsection{Antibody-based biosensors}

Antibodies dominate the biosensor field because of their analytical performance and wealth of methods available for detection of proteins and small molecules directly in complex samples. Generation of antibodies is achieved through repetitive immunization of an animal with a target of interest. ${ }^{2,26}$ Thus, production of antibodies often requires several months of development and there exist prevalent batch-to-batch variability and experimental reproducibility concerns that have been well-documented in several articles. ${ }^{5,27}$ Binding interactions are often described in terms of a dissociation constant -wherein the smaller the value, the stronger the interaction- with antibodies demonstrating dissociation constants of 1 - 1,000 picomolar $\left(10^{-12}\right) \cdot{ }^{27}$ Thus, antibody-based sensors are highly sensitive.

Once isolated, antibodies can be used in a variety of methods to couple the binding interaction with an appropriate transduction mechanism. Small molecule detection requires exogenous labeled-targets that act as signal markers and compete with the free target for antibody binding sites. ${ }^{28}$ Thus, detection of a small molecule analyte requires multiple steps and relatively high-cost. Commercial assays are available for small molecules with assays including competitive enzyme-linked immunosorbent assay (ELISA), homogenous enzyme linked immunoassays, and kinetic interaction of microparticles in solution (KIMS).

\subsubsection{Competitive enzyme-linked immunosorbent assay (ELISA)}

All variations of ELISA assays require competition between a labeled-target or an immobilized target with free target in the test sample for a limited number of antibody binding sites. ${ }^{29}$ For example, the small molecule target can be passively 
immobilized onto a plate surface. The unknown sample is mixed along with a limited amount of the appropriate antibody and incubated in the microplate well. Competition between the free target and the immobilized target will determine the amount of antibody that is specifically absorbed onto the plate wall by binding to the immobilized drug. ${ }^{30}$ If there is no drug present in the sample, most of the antibody will bind to the well of the plate. If drug is present, the antibody will be bound to the free drug and be discarded during the washing steps that remove nonspecifically bound molecules. To quantify the amount of antibody left on the plate wall, a secondary enzyme-labeled antibody is utilized with affinity for the first antibody and an enzyme label such as horseradish peroxidase (HRP), which can convert a substrate to give a signal..$^{29,31}$ Several substrates are compatible with $\mathrm{HRP}$, and the absorbance of the solution can be read with a plate reader to determine the drug concentration. The disadvantages of the assay are the long incubation times required and the necessity for multiple washing steps.

\subsubsection{Homogenous enzyme-linked immunoassays}

These immunoassays utilize an enzyme-linked target and the inhibition of the enzyme-linked target activity by antibody binding. ${ }^{32} \mathrm{~A}$ drug of interest is labeled with an enzyme such as glucose-6-phosphate dehydrogenase (G6PDH), and mixed with the sample of interest where the enzyme-labeled drug and the free drug compete for a limited number of antibody binding sites. ${ }^{33}$ The drug concentration is measured in terms of enzyme activity as it is modulated by binding to the antibody relative to the concentration of free drug in the sample. When displaced by free target binding to the antibody, the active enzyme converts nicotinamide 
adenine dinucleotide (NAD) to $\mathrm{NADH}$, resulting in a change in the absorbance of the solution that can be measured..$^{33}$ The assay displays great sensitivity and relatively fast detection but might suffer from cross-reactivity with off-target molecules and high cost. ${ }^{33}$

\subsubsection{Kinetic interaction of microparticles in solution (KIMS)}

Microparticles are covalently modified with the drug of interest. In solution, these microparticles are dispersed and do not block transmission of light. However, if drug specific antibodies are added to the solution, it results in aggregation of the microparticles because of organized lattice formation by binding interactions between the antibodies and the drug-conjugated microparticles. ${ }^{7}$ The aggregated particles effectively block light transmission through the sample, resulting in increased absorbance. ${ }^{7}$ A sample that does not contain the drug of interest will not change the absorbance of the sample. Samples that are drug positive will inhibit the formation of the aggregated particles by competition for antibody binding sites producing changes in the absorbance.$^{34}$ The absorbance difference before and after addition of sample can be utilized to calculate the drug content. ${ }^{34}$

\subsection{Aptamers}

Aptamers are chemically generated oligonucleotide-based recognition molecules which mimic the binding specificity of antibodies for specific targets with highaffinity interactions but offer low-cost production, high reproducibility and ease of chemical functionalization. ${ }^{39}$ Design of an aptamer-based biosensor from scratch requires generation of an appropriate aptamer and adaption to a signal 
transduction mechanism. Aptamers have thus far been used to design biosensors for small molecules, proteins, cells and ions. ${ }^{8}$ An incredible number of analytical methodologies have been utilized in concert with aptamers to produce signaling including electrochemistry, fluorescence, color-change, and mass-change techniques. ${ }^{35-37}$

\subsubsection{Discovery of aptamers and SELEX}

The isolation of aptamers was first described by two independent research groups in 1990. Tuerk and Gold termed the process of isolating such molecules as SELEX (Systematic Evolution of Ligands by Exponential Enrichment) and Ellington and Szostak coined the term aptamer -the output of SELEX (derived from Greek word meaning to fit). ${ }^{38,39}$ The first SELEX experiment consisted of mutating part of a sequence with a random 8-nt loop (each nucleotide position has a distinct nucleotide A, T, C or G; thus $4^{8} \sim 65,000$ distinct sequences in the library). The library of sequences was utilized to perform multiple rounds of competitive binding and amplification of binding-competent sequences. The result was two sequences that could bind the target from the starting library of $65,000 .{ }^{39}$ While initially met with skepticism about the adaptability to other targets, SELEX has proven to be a reliable method to isolate DNA/RNA molecules that can recognize several types of targets including small molecules, proteins, and small ions. ${ }^{40,41}$

\subsubsection{Systematic evolution by exponential enrichment (SELEX) ${ }^{42-45}$}

The SELEX process consists of various steps to isolate a single binding sequence from a rich and extensive set of unique aptamer sequences (termed a library). The 
library is made up of distinct oligonucleotide molecules (between 10 $10^{13-15}$ ) with constant primer regions (for amplification) and a variable region of random nucleotides that vary in length to increase or fix molecular complexity (for binding interactions). ${ }^{8}$ The library is incubated with the target (i.e., molecule of interest), allowing for binding interactions between the aptamers and the target. The target will naturally have an affinity for certain sequences that exhibit the maximum interaction with the target based on the DNA fold or structure. ${ }^{26}$ The sequences which bind the target are then separated from sequences that do not bind. Separation can be accomplished by various mechanisms and is one of the main differential factors of SELEX technologies. Separation can be achieved by immobilization of the target onto a surface (see figure 2). The library is incubated through a target-immobilized column and the binding aptamers are retained on the column. Non-binding sequences are washed out and discarded while retained aptamers are specifically eluted by addition of high concentrations of free target. For small molecules, the immobilization utilizes an important functional group which might prevent display of all functional groups of the target to the aptamer library. To overcome the issue of immobilizing the target, the library can be immobilized instead by hybridization to a column-conjugated complementary strand. ${ }^{45}$ Addition of the target of interest results in displacement only of aptamers that can undergo target-induced structure-switching from the hybridized conjugated strand to a target-bound complex. The rest of non-binding and immobilized aptamers can be discarded. The separation step is crucial as any nonbinding sequences must be discarded. Following separation, the remaining 
binding sequences are amplified by polymerase chain reaction (PCR). The PCR reaction relies on enzymes to make copies of the remaining sequences in the library. The binding sequences are amplified so the new library can have multiple copies of a singular sequence, referred to as an enriched library. The PCR process produces ds-DNA that must be separated into ss-DNA before the second round of incubation with the target. Separation can be accomplished by primers that are specifically labeled with affinity reagents such as biotin or with phosphate end groups that are resistant to nuclease digestion. The enriched library of ss-DNA can once again be incubated with the target. The same steps are followed for various rounds of competitive binding until only the best binding sequences are present in the enriched library. After each round of SELEX, elution profiles can be utilized to determine whether the enriched library has increased in affinity to the target as compared to the starting library and the library in the previous round. Thus, SELEX has been regularly termed an artificial evolutionary process. Depending on the SELEX methodology and the separation efficiency between binders and nonbinders, this can be from three to thirty rounds. ${ }^{8,17}$ Once the library has been enriched, the remaining winning sequences are cloned and sequenced to determine the exact nucleotide composition. The selection process can be done completely in vitro, without requirements for animal immunization. ${ }^{27}$ Additionally, the specificity of the aptamer can be carefully controlled by removal of sequences which bind to undesirable molecules. The process yields aptamers that have high specificity and sensitivity that can be applied for use in biosensors with unmatched reproducibility if purity and reaction conditions are accurately controlled. 


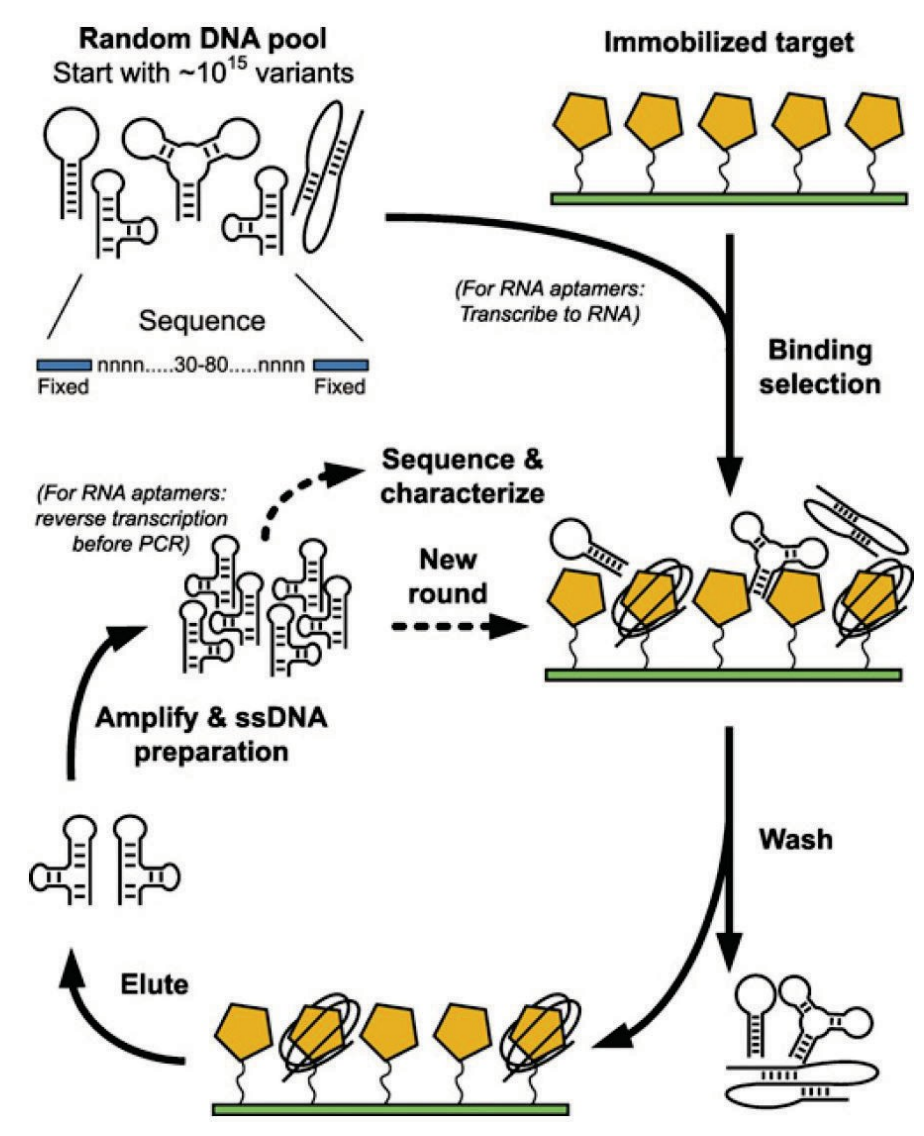

Figure 2. Example schematic of target-immobilized SELEX. The target molecule is immobilized on a surface and incubated with the DNA library. Non-binders are washed out and the binders are eluted specifically and amplified via PCR. The enriched library is utilized for a second round of separation. The selection rounds are repeated until library enrichment is observed by affinity measurements after each round. Once satisfactory enrichment has been achieved, the remaining aptamer pool is cloned, sequence and characterized. (Adapted from Ruigrok et al.). ${ }^{26}$

\subsubsection{Aptamer-based sensors for small molecules}

Aptamers have been utilized to measure various types of small molecules including medical biomarkers, drugs of abuse, and therapeutic drug molecules. ${ }^{17}$ Importantly, aptamers can be synthesized or modified with added chemical functionality which instills new properties apart from target recognition such as direct signal transduction upon target binding. ${ }^{8}$ While well established, aptamer 
sensors for small molecules still suffer from insufficient sensitivity, inconsistent specificity and difficulty in detection of analytes in complex samples. ${ }^{46}$ Aptamers for small molecules generally exhibit equilibrium dissociation constants in the micromolar range, about 100 -fold lower as compared to antibodies. ${ }^{8,27}$ Thus, the sensitivity of aptamer-based sensors is limited by the intrinsic aptamer affinity to the targets and lack of appropriate transduction mechanisms that can maximize the native affinity instead of reducing it. Additionally, several widely utilized small molecule aptamers, such as the ATP-binding aptamer, exhibit cross-reactivity with molecules that are simultaneously present in biological samples, preventing target quantitation in biological samples. ${ }^{47}$ Finally, new methods are needed for universal adaptation of currently reported aptamers to transduction mechanisms which can function in complex matrices. Current methods rely on difficult, laborious and expensive trial-and-error methodologies that are not convenient or universally applicable. ${ }^{17,48}$

\subsection{Cocaine}

Cocaine is an illicit drug under the Controlled Substances Act with a designation of schedule II; this designation is reserved for drugs that have little medical use and an extremely high potential for abuse ${ }^{49,50}$ Cocaine is a small molecule $(<300$ daltons) that still ranks as one the highest illegally consumed and trafficked drugs in the world, according to the United Nations Office of Drug and Crime. ${ }^{51}$ Cocaine affects individuals by inhibiting the reuptake of neurotransmitters in the reward pathway such as dopamine. Drug effects produce exhilaration, euphoria, increased heart rate, rage, and others. ${ }^{52,53}$ Apart from the wanted drug effects, 
cocaine can cause severe health difficulties after prolonged and repeated consumption such as organ failure. ${ }^{54}$ Thus, cocaine remains one of the most tested drugs in law enforcement, criminal investigations and drug withdrawal programs. Cocaine is quickly metabolized upon consumption with the major metabolite in urine being benzoylecgonine. ${ }^{55,56}$ Benzoylecgonine is present in urine at concentrations $>100 \mathrm{mg} / \mathrm{L}$ for up to 24 hours after consumption while cocaine is present at levels $>65 \mathrm{mg} / \mathrm{L}$ in urine within 8 hours and at trace levels thereafter. ${ }^{56}$ Cocaine can also be found in saliva and serum at concentrations $<0.4 \mathrm{mg} / \mathrm{L}$. Thus commercial immunoassays detect benzoylecgonine in urine rather than cocaine as a presumptive test of cocaine use..$^{33,56}$

\subsubsection{Cocaine metabolite detection with antibodies}

Various antibody-based assays are commercially available and widely employed with a positive result cut-off level of $0.3 \mu \mathrm{g} / \mathrm{L} .{ }^{33}$ The EMIT II assay employs a glucose-6-phosphate dehydrogenase (G6PDH) enzyme conjugated to benzoylecgonine and benzoylecgonine specific antibodies for detection. ${ }^{32}$ If the cocaine metabolite is present in a biological sample, the free benzoylecgonine will compete for binding with the G6PDH-linked benzoylecgonine. The displacement of G6PDH-linked benzoylecgonine leads to reactivation of enzymatic activity and transformation of nicotinamide adenine dinucleotide (NAD) to $\mathrm{NADH}$, resulting in a change in the absorbance of the solution that can be measured via spectroscopy.${ }^{33}$ Competitive ELISA can also be employed for cocaine metabolite detection. The method consists of immobilizing benzoylecgonine specific antibodies onto a microplate well. A mixture of enzyme-labeled benzoylecgonine 
is then incubated on the microplate well with the tested sample. Competition for the immobilized antibody binding sites is utilized to calculate the percent of labeled drug that binds to the immobilized antibody. Other immunoassays for cocaine metabolite rely on similar competition mechanisms between labeled benzoylecgonine and free benzoylecgonine in the sample matrix. These immunoassay methods exhibit cross-reactivity that depends on the specificity of the antibody and are susceptible to batch-to-batch variation. Additionally, the antibodies must be carefully stored and transported and exhibit limited shelflife.

\subsubsection{Cocaine detection with aptamers}

The cocaine aptamer was isolated by Milan Stojanovic and co-workers in $2000 .{ }^{57}$ The original aptamer was termed MNS-4.1 and had a three-way junction secondary structure. The three-way junction is a description of three distinct intramolecular helices present around a central target-binding pocket. It has been postulated that the binding pocket at the junction of the helices is a highly hydrophobic environment because of unstacked nucleotides at the terminal of each junction displaying hydrophobic surfaces to the internal pocket. ${ }^{58}$ The isolated cocaine-binding aptamer has been utilized in several major sensor categories: (1) as a fully stable and singular three-way junction aptamer in dyedisplacement assays, (2) as a singular, flexible aptamer in structure-switching assays and (3) as a split structure wherein the aptamer is a composed of two separate strands that can hybridize in the presence of cocaine..$^{57,59,60}$ The latter two categories suffer from limited sensitivity as it has been experimentally determined that destabilizing or splitting of the aptamer structure reduces the 
binding affinity for cocaine ${ }^{61,62}$ For example, Stojanovic et al. initially demonstrated a fluorescence sensor by splitting the aptamer to produce target-induced selfassembly of the complementary fragments. One fragment was labeled with a fluorescent molecule while the other fragment was labeled with a quencher molecule. When cocaine was not present, the two fragments existed separately and thus a high fluorescence signal could be measured. Cocaine would induce self-assembly of the two fragments and bring the quencher near the fluorescent molecule, turning off the fluorescent signal. In this way, cocaine could be detected and differentiated from highly similar molecules, including benzoylecgonine which varies at a single functional group. Unfortunately, splitting of the aptamer reduces binding affinity for cocaine, limiting the sensitivity of the sensor. Stojanovic and coworkers later developed a more sensitive cocaine assay by stabilizing the threeway junction structure for a high affinity interaction with cocaine. They discovered that the colorimetric dye Cy7 could bind to the binding pocket in the absence of cocaine but was quickly displaced upon cocaine-aptamer specific binding, resulting in change of the color of the solution. ${ }^{60}$ In this circumstance, the affinity of the aptamer was conserved, leading to higher sensitivity as compared to the split aptamer design. Thus, an important structural feature for cocaine sensing is conserving or improving the high affinity interaction and coupling it with a sensitive transduction mechanism. However, the strategy has not been regularly reported with most transduction systems using cocaine-induced structure-switching including electrochemistry, fluorescence, absorbance, and microcantilevers. ${ }^{60,63,64}$ 


\subsection{3 (2)-amino-5,6,7-trimethyl-1,8-naphthyridine (ATMND)}

The small molecule ATMND is a fluorescent dye that was developed for DNA single nucleotide polymorphism (SNP) typing. The ATMND molecule was specifically designed to bind to nucleobases opposite an abasic site (a space where a nucleotide is missing) by pseudo-hydrogen bonding to the unpaired nucleobase opposite the abasic site and stacking interactions with the neighboring nucleotides. ${ }^{65}$ Formation of hydrogen bonds and stacking in the DNA helix affects the fluorescence of the molecule. Free in solution, ATMND is highly fluorescent when excited at $358 \mathrm{~nm}$. However, when bound inside the DNA helix, ATMND is non-fluorescent. ${ }^{66}$ The base opposite the abasic site determines the binding affinity of ATMND, with strong binding with cytosine (C) and thymine (T) but not but Adenine or guanine ( $A$ and $G) \cdot{ }^{67}$ Abasic sites can be artificially incorporated onto synthesized DNA or be mimicked by hybridization of DNA that leaves an empty base site in the sequence, thus aptamers can be functionalized with a high-affinity binding site for ATMND. ${ }^{68-70}$ Accordingly, ATMND has been utilized in various aptamer-based assays as a fluorescence reporter ${ }^{69-71}$ For example, Xiang et al. developed an aptamer-sensor where ATMND is initially bound to an artificial abasic site by hybridization of an aptamer sequence with a short-competing oligonucleotide molecule. Target binding results in displacement of the hybridized oligo, thus disrupting the binding site and displacing ATMND form the aptamer. The fluorescence of the displaced ATMND can be correlated to the target concentration. ${ }^{69,71}$ Additionally, ATMND was also found to bind to C-C mismatched nucleotides in DNA stems, a property that has been exploited for detection of 
analytes via displacement of ATMND from the $\mathrm{C}-\mathrm{C}$ binding site ${ }^{72}$ These strategies require chemical modification of the aptamer or addition of an anti-sense strand. Modification of the aptamer reduces the inherent binding affinity of the aptamer and limits the sensitivity of the assay.

\subsection{Adenosine}

Adenosine is an endogenous molecule present throughout the body with extensive roles in extracellular signaling pathways. ${ }^{73}$ Adenosine is primarily but not exclusively produced by metabolism of adenosine triphosphate, adenosine diphosphate and adenosine monophosphate outside of the cell by transformation of ATP to AMP by the enzyme CD39 followed by transformation of AMP to Ade by CD73. ${ }^{74}$ Outside the cell, adenosine signaling functions by binding to four distinct adenosine specific receptors: $A_{1}, A_{2 a}, A_{3}$, and $A_{2 b}{ }^{73}$ Adenosine is the main agonist of these receptors, initiating signaling pathways that affect immune response, inflammation, blood flow and neurotransmitter release. Adenosine has been found at physiological concentrations of $30-300 \mathrm{nM}$ under physiological conditions but at concentrations above $10 \mu \mathrm{M}$ under pathological conditions (i.e., disease states) such as cellular stress, cell damage, hypoxia and cancer. ${ }^{73,75-77}$ In cancer pathogenesis, adenosine can act as an immunosuppressive signaling molecule by binding to the $\mathrm{A}_{2 a}$ receptor. ${ }^{78}$ It has been shown experimentally that mice lacking this receptor can recuperate from tumors, indicating the role of adenosine in actively preventing activation of tumor preventing cells. As the role of adenosine in immune response and inflammation needs to be explicitly understood, improved tools to measure adenosine concentration are urgently needed. 


\subsubsection{Adenosine concentration measurements}

Current methods to measure adenosine concentration in biological samples include high performance liquid chromatography (HPLC), capillary electrophoresis (CE), micro-dialysis, and enzyme-based sensors. ${ }^{76,77,79}$ For example, HPLC can readily detect adenosine with a simple absorbance detector as adenosine has a distinct absorbance. More recently, HPLC has been coupled with mass spectrometry to produce lower limits of detection and ability to measure more complex samples. ${ }^{80}$ However, such methods cannot be used for real-time detection, and also require complex sample handling and instrumentation. Adenosine is quickly metabolized or transported inside of cells, thus real-time monitoring of adenosine concentration might be better suited for more accurate diagnostics. ${ }^{75}$ Additionally, adenosine concentration might vary close to the cell surface, where adenosine is commonly produced. Thus, cell anchored recognition elements might provide accurate concentration measurements of adenosine, however such methods do not currently exist. The closest direct sensor for adenosine is by use of enzymatic recognition. These sensors utilize enzymes that can catalyze the transformation of adenosine into inosine, xanthine and other by products eventually leading to the production of hydrogen peroxide, which can be readily detected using electrochemistry or spectroscopy. ${ }^{81}$ Immobilization of these enzymes inside a membrane bound to an electrode surface provides a sensor methodology that can readily measure adenosine with much higher temporal resolution and can be surgically implanted to measure adenosine in situ. Unfortunately, insertion of these sensors into tissue causes damage that results in 
release of ATP and thus affects the actual physiological concentration of adenosine. These sensors are also prone to interference from endogenous molecules such as inosine and xanthine, which are commonly found in biological fluids. ${ }^{81,82}$

\subsubsection{Aptamer-based adenosine measurements}

Huizenga et al. isolated the ATP-binding aptamer in $1995 .{ }^{47}$ The aptamer can bind to multiple adenosine derivatives including adenosine, adenosine monophosphate, adenosine diphosphate, and adenosine triphosphate. ${ }^{47,83}$ Unfortunately, these molecules are simultaneously present in biological fluids. ${ }^{84,85}$ Therefore, the current ATP-binding aptamer cannot be utilized for specific determinations of adenosine concentration in complex samples or clinically relevant diagnostics. Most publications have utilized the ATP-binding aptamer as a test bed for a transduction method rather than attempting to address the issue of specificity. For example, Lu et al. developed a sensor that used time-resolved luminescence for detection of adenosine in undiluted serum samples. ${ }^{46}$ However, the method displayed cross-reactivity with adenosine phosphate derivatives and displayed limited sensitivity $(60 \mu \mathrm{M})$. Only one reported aptamer structure is specific for adenosine against negatively charged phosphate derivate molecules.

Stojanovic et al. isolated an adenosine binding aptamer that was engineered to provide significant binding affinity for adenosine and decrease affinity with linear phosphate derivatives because of steric repulsion as argued by the authors. However, the Stojanovic aptamer structure has not been studied in detail and its application for complex sample measurements has not been determined. ${ }^{86}$ 


\subsubsection{Aptamer engineering}

An important aspect of aptamer-sensor development is the need to instill signal reporting functionalities to aptamers and to fine-tune the affinity and specificity of the aptamer. Aptamer engineering can include mutation of nucleotides in the sequence, addition of nucleotides or removal of non-essential nucleotides. ${ }^{47}$ Several examples in the literature have demonstrated increased aptamer performance after aptamer engineering. ${ }^{48,86,87}$

\subsubsection{Electrochemical aptamer-based (E-AB) sensors}

Electrochemical detection of molecules by linking with aptamer recognition is an extremely powerful technology that can be utilized in complex samples such as whole blood. ${ }^{10,88}$ The technique was inspired by electrochemical detection of DNA via immobilized DNA probes mimicking molecular beacons that were covalently labeled with a ferrocene tag which is an electroactive molecule. ${ }^{89}$ In the absence of DNA target, the immobilized stem-loop probe keeps the ferrocene tag near the electrode surface, with large currents being measured. Binding of the immobilized probe to a target DNA resulted in formation of double-stranded DNA structures that separated the electroactive group from the electrode surface. The distance directly affected the rate of electron transfer between the electroactive molecule and the electrode surface, thus the change in current could be used to determine the amount of DNA bound to the immobilized probe. ${ }^{89}$ Soon thereafter, aptamers were immobilized onto electrode surfaces (via strong thiol-gold bonds) and covalently labeled with methylene blue, an electroactive molecule. ${ }^{90}$ Xiao et al. first developed a signal-off sensor where the immobilized aptamer was separated from the 
electrode surface by binding to thrombin (a relatively large molecule), resulting in reduced mobility of the aptamer and a reduction in the measured current. ${ }^{91}$ The native aptamer structure can also be engineered to maintain a flexible and open conformation which places the electroactive molecule far from the electrode surface, inhibiting the transfer of electrons. ${ }^{92}$ Target-binding can induce aptamer folding around the target causing the electroactive molecule to approach the surface and increase the rate of electron transfer. Thus, the current measured increases as target is added to the solution. ${ }^{93} \mathrm{An}$ important aspect of E-AB sensors is the preparation of the aptamer-immobilized electrode. ${ }^{94}$ The aptamer is immobilized by addition of a thiol chemical group to one end of the aptamer. Gold electrodes can then form strong bonds with the thiol group, causing immobilization of the aptamer to the gold surface. ${ }^{95}$ While the thiol-gold bond is very strong, the nitrogenous bases of the DNA sequence are known to weakly interact with the gold surface. ${ }^{96}$ Thus, short alkane-thiol molecules can be added to prevent adsorption of the nitrogenous bases to the electrode surface.${ }^{95}$ These short molecules form strong interactions with the gold surface and organize in neat monolayers because of hydrophobic interactions between the alkane chains of the immobilized molecules. Their role is to keep the aptamers in a 'standing' direction by preventing absorption of the nitrogenous bases to the electrode surface and to carefully control the density of immobilized aptamers at the electrode surface. ${ }^{95,97,98}$ Once prepared, the aptamer-bound electrodes can be utilized repeatedly by directly submerging into a sample of interest. A voltage is applied to the electrode to interrogate the electroactive molecule and measure the resulting current. 


\subsection{Aptamer structure-switching}

Aptamer isolation techniques often utilize libraries that have highly stable secondary structures with static conformations, requiring substantial testing to affirm or instill structure-switching upon target binding. ${ }^{17,48,62,83}$ Lack of structural change prevents adaptation to signaling mechanisms, as most require significant differences between the target-free structure and target-bound structure. Aptamer truncation describes a general process of removing nucleotides in the aptamer sequence that are not essential for binding, destabilizing the structure and instilling structure-switching functionality to the truncated aptamer. ${ }^{48,62,99}$ Aptamer truncation can be attained by manually removing bases from various parts of the aptamer to deduce the primary binding site but it is laborious and expensive because it requires characterization of multiple aptamer derivatives. Generally, most techniques rely on similar trial-and-error mechanisms until a short aptamer sequence is identified. ${ }^{17,100,101}$ For example, the cocaine aptamer was previously engineered to destabilize the target-free structure, with aptamer folding only occurring when cocaine was bound to the aptamer. ${ }^{102}$ The signaling occurred through a fluorescent label that was attached to one end of the aptamer and a quenching molecule attached to the opposite end. In the absence of cocaine, the two molecules were spatially separated but upon target-binding the molecules were in proximity. Thus, cocaine could modulate the fluorescence signal although affinity was compromised because of destabilization of the aptamer binding site. While functional, this technique is not easily adapted to other aptamers, requiring extensive work to deduce the target binding site and the minimum number of 
nucleotides to provide binding affinity to the target. Therefore, other ways have been developed to instill structure-switching functionality. For example, a short complementary DNA strand can be used to hybridize with part of the aptamer sequence and block the binding site of the analyte. ${ }^{83}$ The length of the competing sequence is carefully designed to allow target-induced displacement. If the length of the competitor sequence is too long, the target would not be able to displace the competitor. While this method is easier to use with various aptamers, there is severe reduction in the affinity of the aptamer to the target because of the inherent competition imposed by the competing DNA strand. ${ }^{46,83}$

\subsubsection{Nuclease-guided truncation of aptamers}

Several purified enzymes are commercially available for a wide range of applications in transformation of DNA molecules. These enzymes often display incredible specificity for a specific structure of DNA, such as double-stranded DNA or single-stranded DNA. Thus, these enzymes can be utilized to probe the changes in the DNA conformation upon target-binding.

\subsubsection{Exonuclease I (Exo I)}

Exonuclease I is an enzyme purified from $E$. coli with a high level of specificity for hydrolysis of single-stranded DNA but with little to effect on double-stranded DNA. ${ }^{103}$ The enzyme can hydrolyze ss-DNA from the 3'- end stepwise, releasing single mononucleotides. The enzyme requires a divalent cation for catalysis and optimally performs under basic conditions. The enzyme has been previously utilized to detect small molecules with aptamer recognition. ${ }^{104}$ In the absence of 
target, the aptamers have a conformation that is hydrolyzed by Exo I however upon binding to the target, the aptamer-target complex is not susceptible to enzymatic digestion. Addition of SYBR gold DNA binding dye was utilized to measure the amount of aptamer product left and correlated with the concentration of target.

\subsubsection{Exonuclease III (Exo III)}

Exonuclease III is an enzyme purified from $E$. coli that can catalyze the hydrolysis of various types of DNA. Exonuclease III can perform activities such as digestion of double-stranded DNA from the 3'- end of DNA, at blunt ends or at recessed 3'ends but is not able to digest 3'- extensions longer than 4 nucleotides. ${ }^{105,106}$ The digestion results in release of mononucleotides and formation of single stranded DNA. Exonuclease III also displays endonuclease activity at abasic sites, resulting in nicks of the DNA sequence and formation of separate DNA fragments, and phosphatase activity for cleaving phosphate 3' ends to 3'-OH, as well as Rnase H activity for catalyzing the hydrolysis of RNA/DNA hybrids. ${ }^{107,108}$

Exonuclease III has been employed to truncate aptamer sequences to yield structure-switching aptamers and also to determine the binding sites of proteins to DNA. Such footprinting studies were based on the inhibition of the enzyme as it approached the protein-DNA binding site, producing leftover DNA fragments that could be visualized via gel electrophoresis to approximate the binding site by comparison to a control sample. ${ }^{109} \mathrm{~A}$ similar inhibition effect was discovered by Wang et al. where formation of aptamer-target complexes can inhibit the hydrolysis of aptamers by exonuclease III 4-nt away from the presumed binding site. In the 
absence of binding target, exo III can completely digest the aptamer molecule. Furthermore, the yielded truncated aptamer sequences were shown to undergo target-induced structure-switching. The method demonstrated a general and simple platform to truncate stable, folded aptamers to yield structure-switching capable aptamer sequences. ${ }^{110}$

\subsubsection{T5 exonuclease}

The enzyme T5-exonuclease can preferentially digest 5' overhangs, flaps and linear single- and double-stranded DNA in the 5' to $3^{\prime}$ direction. ${ }^{111}$ The enzyme structure has been previously elucidated, with a small opening that is big enough to allow the threading of single-stranded DNA but not double-stranded DNA. ${ }^{112}$ The enzyme T5 can bind to a 5' overhang and thread the single-stranded sequence through the active site arch until reaching a junction of double-stranded DNA. The enzyme then hydrolyzes the threaded DNA resulting in a blunted dsDNA and a single-stranded fragment. The active site of the enzyme is lined with hydrophobic residues that presumably stabilize the threading of single-stranded DNA, and binding of divalent ions at the active site that mediate the hydrolysis reaction. ${ }^{113} \mathrm{~A}$ variety of divalent metal ions can be used as cofactors of T5 exonuclease, with different ions modulating the activity of the enzyme. ${ }^{114} \mathrm{T5}$ exonuclease has been described as a complementary enzyme to exonuclease III, which has similar activity but in the 3' to 5' direction. Exonuclease III has been utilized for determination of the binding site of molecules to DNA, thus it is possible that T5 exonuclease could perform a similar function. 


\subsubsection{Methylenedioxypyrovalerone (MDPV)}

The drug MDPV is an illicit substance under the Controlled Substances Act. It is listed as a schedule I drug with a high potential for abuse and no known medical use. The drug was initially part of the 'bath salt' epidemic, commonly sold as a product not meant for human consumption at convenience stores or over the Internet. ${ }^{115}$ The effect of MDPV is mediated by inhibiting dopamine reuptake and thus creating exhilaration with similar effects as drugs like cocaine and amphetamine. ${ }^{116}$ The common dosage of MDPV is in the milligram range and can be consumed in various ways such as snorting or smoking. Long term effects apart from addiction are not widely known although like cocaine, inhibition of the reward uptake pathway can have damaging health consequences. ${ }^{117}$ Detection of MDPV is an increasing concern for law enforcement and health administration. Antibodybased tests are available for screening of MDPV, with common confirmatory methods including GC/MS and other chromatography-mass spectrometry methods. ${ }^{118,119}$ However, MDPV is part of a family of drugs that have been extensively modified to avoid detection. Unfortunately, current immunoassays only recognize few structures thus new assays are urgently needed to screen the drug family rather than a single molecule specifically. ${ }^{120}$

\subsubsection{Adenosine triphosphate (ATP)}

Adenosine triphosphate (ATP) has various roles in cell signaling such as inflammation and immune response.${ }^{74}$ Adenosine triphosphate is present intracellularly (i.e., inside the cell) at relatively high concentrations, between 5-10 $\mathrm{mM} .{ }^{121}$ The ATP molecule is amphiphilic, with hydrophobic groups as a result of 
the nitrogenous base and hydrophilic because of the highly charged phosphate groups. A recent report suggest that the high intracellular concentration of ATP is because of the role of ATP as a hydrotrope, a compound that can facilitate solubilization of hydrophobic compounds. ${ }^{121}$ Adenosine triphosphate is transported to the outside of the cell by specific channel transporters and exocytosis. ${ }^{122}$ Outside the cell, it effects its signaling function by binding to a wide range of ATP receptors (termed P2 receptors). ${ }^{123}$ Its cellular signaling affects mechanisms such as inflammation and immune response. ATP can be reliably measured by the luciferase reaction. Luciferase can utilize ATP to oxygenate luciferin, producing light as a result. ${ }^{84}$ ATP concentration can be measured by the amount of light that is produced. More recent reports have outlined a method to immobilize this enzyme to cell membranes, permitting in situ measurements of ATP concentration in real time. ${ }^{124}$ Aptamer-sensors have also been designed for specific detection of ATP albeit with high dissociation constants and crossreactivity with ADP. ${ }^{125}$ 
Adapted with permission from Analytical Chemistry. 86, 22, 11100-11106. Copyright@ 2014 American Chemical Society

\section{CHAPTER 3}

\section{A Label-Free Aptamer-Fluorophore Assembly for Rapid and Specific Detection of Cocaine in Biofluids}

We report a rapid and specific aptamer-based method for one-step cocaine detection with minimal reagent requirements. The feasibility of aptamer-based detection has been demonstrated with sensors that operate via target-induced conformational change mechanisms, but these have generally exhibited limited target sensitivity. We have discovered that the cocaine-binding aptamer MNS-4.1 can also bind the fluorescent molecule 2-amino-5,6,7-trimethyl-1,8-naphthyridine (ATMND) and thereby quench its fluorescence. We subsequently introduced sequence changes into MNS-4.1 to engineer a new cocaine-binding aptamer (38GC) that exhibits higher affinity to both ligands, with reduced background signal and increased signal gain. Using this aptamer, we have developed a new sensor platform that relies on the cocaine-mediated displacement of ATMND from 38-GC as a result of competitive binding. We demonstrate that our sensor can detect cocaine within seconds at concentrations as low as $200 \mathrm{nM}$, which is 50 -fold lower

than existing assays based on target-induced conformational change. More importantly, our assay achieves successful cocaine detection in body fluids, with a limit of detection of $10.4,18.4$, and $36 \mu \mathrm{M}$ in undiluted saliva, urine, and serum samples, respectively. 


\subsection{Introduction}

Cocaine is a central nervous system stimulant that increases levels of dopamine ${ }^{126,127}$ and potently inhibits neurotransmitter reuptake at the synapse. ${ }^{128-}$

132 Abuse of cocaine has been shown to cause anxiety, paranoia, mood disturbances, organ damage, and violent behavior. ${ }^{52,133,134}$ Therefore, rapid detection of cocaine is needed to confirm suspicion of recent use in impaired driver investigations or to assist in overdose treatment in medical emergency settings. Various immunoassays have been developed for the detection of cocaine and/or its major metabolite benzoylecgonine in biofluids, including the enzyme-linked immunosorbent assay (ELISA) $)^{135}$ and the EMIT II Plus Cocaine Metabolite Assay. ${ }^{136}$ Unfortunately, the use of these assays is often limited because of the high cost of generating antibodies and issues with poor specificity. These antibodybased tests often cannot distinguish between the targeted drug and structurally similar substances, resulting in cross-reactivity-related false positives. ${ }^{137,138}$

Aptamers are single-stranded RNA or DNA molecules selected in vitro via Systematic Evolution of Ligands by Exponential Enrichment (SELEX) ${ }^{39}$ to specifically bind to targets with high affinity, and they offer a practical alternative to antibodies for the detection of nucleic acids, proteins, and small molecules. Compared to antibodies, aptamers are relatively fast and cheap to produce and can be chemically synthesized with extreme accuracy and reproducibility. ${ }^{26,139}$ Aptamer-based sensors have been gaining popularity because of their simplicity and specificity. For example, derivatives of the MNS-4.1 cocaine-binding aptamer ${ }^{57}$ have been labeled with sensing elements such as fluorophore/quencher 
pairs, ${ }^{59}$ magnetic or metallic nanoparticles, ${ }^{11,140,141}$ quantum dots, ${ }^{142,143}$ and methylene blue ${ }^{64,144}$ to achieve specific detection of cocaine. In the absence of cocaine, the aptamer population exists in an equilibrium state consisting of both folded and unfolded structures, ${ }^{62}$ where the folded structures generate a background signal. When challenged with cocaine, the unfolded aptamers undergo a target-induced conformational change and form a noncanonical threeway junction that binds cocaine ${ }^{62}$ producing a signal change. This limited targetinduced fluorescence change resulted in a high detection limit $(10 \mu \mathrm{M})$ even under optimal conditions, and the reason may be due to inefficient proximity quenching, low aptamer-target binding affinity, or both. ${ }^{59}$

Different strategies such as target displacement have been used to increase the sensitivity of aptamer-based detection. For example, Stojanovic's group used unmodified MNS-4.1 (Figure 3, MNS-4.1) to construct a colorimetric cocaine sensor based on cocaine-mediated displacement of a cyanine dye (diethylthiotricarbocyanine iodide; Cy7) from the dye-aptamer complex. ${ }^{60}$ They observed that the absorbance of Cy7 at $760 \mathrm{~nm}$ decreased with increasing cocaine concentrations in the range of 2 to $600 \mu \mathrm{M}$. Compared to the corresponding fluorescence sensor that exhibited less sensitivity due to intrusive covalent modifications on the aptamer, ${ }^{59}$ the sensitivity of the Cy7-based displacement assay was improved due to the high binding affinity of the unmodified MNS-4.1 aptamer for cocaine.$^{59,60,145}$ However, the limit of detection of this sensor was still limited to $2 \mu \mathrm{M}$ as a result of high background signal from the weak interaction between Cy7 and MNS-4.1.60 

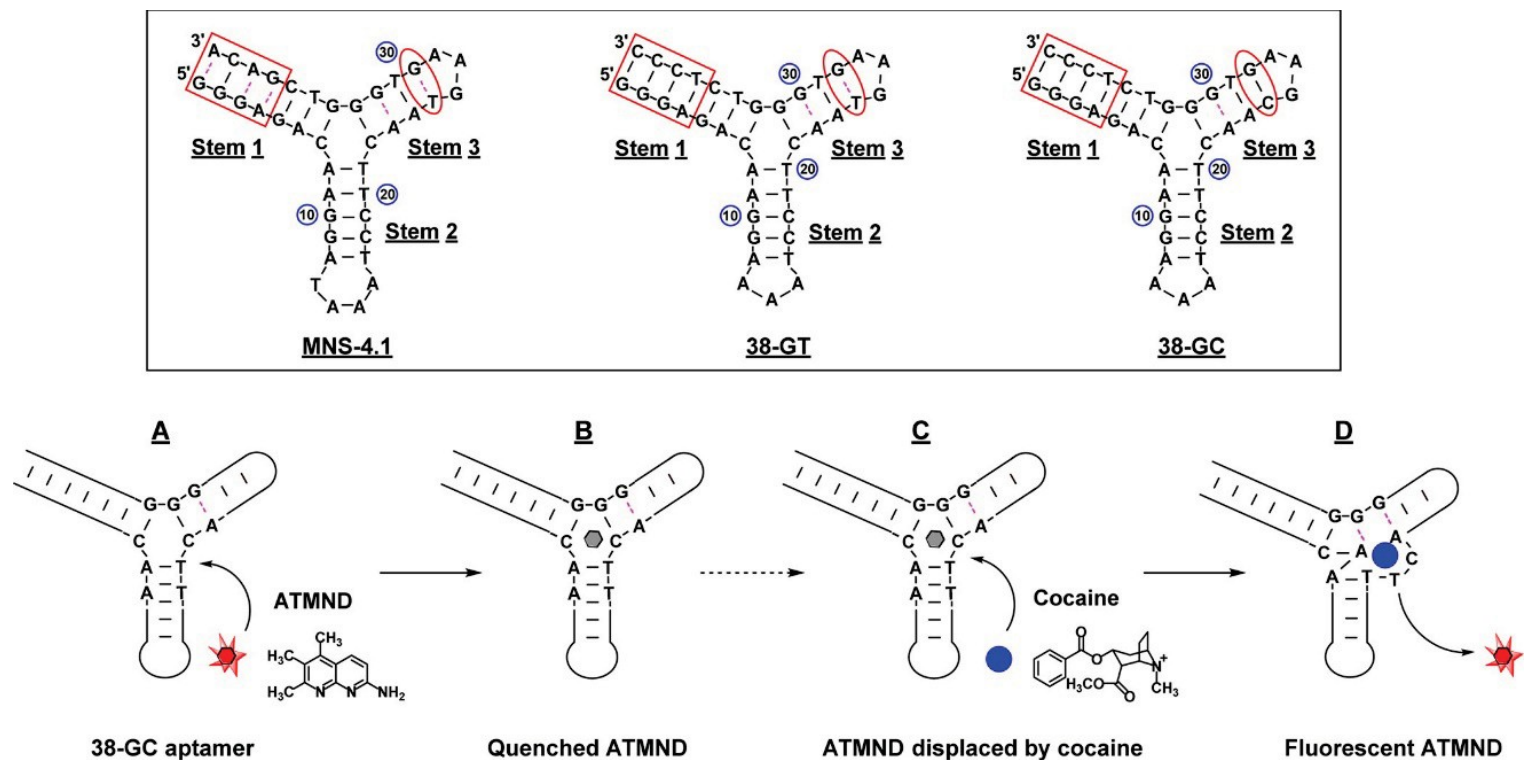

Figure 3. (Top) Structures of the MNS-4.1, 38-GT, and 38-GC aptamers.

These aptamers are predicted to fold into three helical stems built around a threeway junction. Stem 1 of MNS-4.1 contains three putative noncanonical base pairs, which have been substituted for Watson-Crick base pairs in 38-GT. 38-GC was further stabilized by converting the G-T wobble pair in stem 3 to a matched G-C base pair. (Bottom) Scheme of cocaine detection with the 38-GC aptamer sensor. $38-G C$ stably folds in the absence of ligand at room temperature. ATMND binding to the aptamer quenches the molecule's fluorescence, but after challenging this complex with cocaine, the competitive binding displaces ATMND from the binding site. The released ATMND generates a readily detectable fluorescent signal, indicating the presence of the cocaine target.

In this work, we found that the MNS-4. 1 cocaine binding aptamer can also bind the fluorescent dye 2-amino-5,6,7-trimethyl-1,8-naphthyridine (ATMND), quenching its fluorescence. In the presence of cocaine, however, the bound ATMND is competitively displaced, generating a rapid fluorescent readout as the released molecule is highly fluorescent in solution. Based on our characterization of this competitive binding of the cocaine target and ATMND signal reporter to the same aptamer, we further improved the performance of our aptamer sensor by deriving a new variant (Figure $3,38-G C$ ) of MNS-4. 1 that stably assumes a predominantly 
folded conformation at equilibrium. Using this optimized aptamer, we developed a rapid and selective assay for the detection of cocaine at concentrations as low as $200 \mathrm{nM}$ within 20 seconds, $\sim 50$-fold lower than assays based on target-induced conformational change, ${ }^{140}$ but in less time and with fewer reagent requirements. ${ }^{146-148}$ Importantly, our sensor can also rapidly achieve cocaine detection at micromolar concentrations in body fluids such as urine, saliva, and serum.

\subsection{Materials}

ATMND was purchased from Ryan Scientific, and 3,3'-diethylthiatricarbocyanine iodide (Cy7) was purchased from Sigma-Aldrich; any dilution or stock solution was prepared with dimethyl sulfoxide (DMSO). Cocaine hydrochloride was purchased from Sigma-Aldrich, and benzoylecgonine tetrahydrate was purchased from Cerilliant Corporation. Both $50 \mathrm{mM}$ stock solutions were prepared in acidic deionized water solution $(\mathrm{pH} 4.5)$ and stored at $4{ }^{\circ} \mathrm{C}$. All DNA aptamers were ordered from Integrated DNA Technologies with high performance liquid chromatography (HPLC) purification and dissolved to a concentration of $500 \mu \mathrm{M}$ in $1 \times$ filtered Tris-EDTA buffer (10 mM Tris, $1 \mathrm{mM}$ EDTA, pH 8.0). DNA concentrations were measured on a NanoDrop 2000 (Thermo Scientific). DNA sequences are listed below:

- 38-GT: 5' GGG AGA CAA GGA AAA TCC TTC AAT GAA GTG GGT CTC CC 3';

- 36-GT: 5' Gg AGA CAA GGA AAA TCC TTC AAT GAA GTG GGT CTC C 3'; 
- 34-GT: 5' G AGA CAA GgA AAA TCC TTC AAT GAA GTG GGT CTC 3';

- 32-GT: 5’ AgA CAA GGA AAA TCC TTC AAT GAA GTG GGT CT 3';

- 30-GT: 5' GA CAA GGA AAA TCC TTC AAT GAA GTG GGT C 3';

- 38-GC: 5' GGG AGA CAA GGA AAA TCC TTC AAC GAA GTG GGT CTC CC 3';

- 38-GC M1: 5' GGG AGA CAA GGA AAA TCC TCT AAC GAA GTG GGT CTC CC 3';

- 38-GC M2: 5' GGG AGA CAA GGA AAA TCC TAC AAC GAA GTG GGT CTC CC 3';

- MNS-4.1: 5' GGG AGA CAA GGA TAA ATC CTT CAA TGA AGT GGG TCG ATA 3'

\subsection{Methods}

ATMND Binding and Cocaine Displacement Experiments

For cocaine detection, we prepared $96 \mu \mathrm{L}$ of reaction buffer (10 mM Tris, $0.01 \mathrm{mM}$ $\mathrm{MgCl}_{2}, \mathrm{pH} 7.4$ ), $1 \mu \mathrm{L}$ of aptamer (final concentration $2 \mu \mathrm{M}$ ), and $1 \mu \mathrm{L}$ of ATMND (final concentration $0.25 \mu \mathrm{M}$ ) solution. Each $98 \mu \mathrm{L}$ reaction was loaded into one well of a 96-well plate. Fluorescence readings were taken $3 \mathrm{~min}$ apart to determine the stability of the signal, with excitation at $358 \mathrm{~nm}$ and emission at $405 \mathrm{~nm}$. After the signal stabilized for $15 \mathrm{~min}$, a $2 \mu \mathrm{L}$ cocaine solution $(0-2.5 \mathrm{mM})$ was added to each well using a multichannel pipet while monitoring the fluorescence signal. Samples were prepared in triplicate with average values used to plot the figures. 


\subsubsection{Detection with Cy7}

We performed the same set of experiments with the same solutions as described above for ATMND, but with $1 \mu \mathrm{L}$ (final concentration $7 \mu \mathrm{M}$ ) of Cy7 instead of ATMND. The absorbance was recorded at $760 \mathrm{~nm}$.

\subsubsection{ITC Experiments}

ITC experiments were performed with a MicroCal iTC200 instrument (GE Healthcare). All measurements were performed in $10 \mathrm{mM}$ Tris buffer $(\mathrm{pH} 7.4)$ with $0.01 \mathrm{mM} \mathrm{MgCl} 2$ and $5 \%$ DMSO. The sample cell contained the aptamer solution, while the titrant was loaded in the syringe. In order to detect tight binding, the aptamer concentration was kept at $20 \mu \mathrm{M}$ while the titrant (cocaine or ATMND) concentration was $500 \mu \mathrm{M}$. We introduced 19 total injections of $2 \mu \mathrm{L}$ each and a purge injection of $0.4 \mu \mathrm{L}$. The purge injection was not included in the calculations. Two sets of each experiment with cocaine or ATMND were performed at $25^{\circ} \mathrm{C}$. The raw data were averaged and fitted to the two sets of sites and single-site binding models and adjusted for the heat of the titrant.

\subsubsection{Detection of Cocaine in Urine, Serum, and Saliva}

Different concentrations of biofluids were obtained by dilution with deionized water. To test the quenching effect of biofluids, we mixed ATMND $(500 \mathrm{nM})$ in $2 \times$ reaction buffer (20 mM Tris, $0.02 \mathrm{mM} \mathrm{MgCl}$, $\mathrm{pH}$ 7.4) with an equal volume of biofluids, followed by fluorescence measurements. To test the signal gain of cocaine in different biofluid dilutions, we spiked cocaine $(500 \mu \mathrm{M})$ into $50 \%, 25 \%, 10 \%, 5 \%$, and $0 \%$ urine, serum, and saliva. These were then mixed with an equal volume of 
$2 \times$ reaction buffer containing ATMND (500 $\mathrm{nM})$ and 38-GC $(4 \mu \mathrm{M})$, followed by subsequent fluorescence recording. To perform cocaine detection in biofluids, different cocaine concentrations were spiked into $5 \%$ urine, $5 \%$ serum, or $10 \%$ saliva. Equal volumes of cocaine-spiked biofluids and $2 \times$ reaction buffer containing ATMND (500 nM) and 38-GC (4 $\mu \mathrm{M})$ were mixed to read the fluorescence. Unless otherwise indicated, fluorescence measurements were obtained with excitation at $358 \mathrm{~nm}$ and emission at $405 \mathrm{~nm}$.

\subsection{Results and discussion}

We devised our platform based on cocaine-mediated displacement, employing an aptamer sensor that reports the presence of cocaine via the displacement and unquenching of a bound fluorophore molecule. In the absence of ligand, the cocaine binding aptamer forms three helical stems around a three-way junction. ATMND binds the aptamer at this junction, which results in the quenching of its fluorescence (Figure 3B). Since this aptamer also binds cocaine, the competitive binding of cocaine results in a rapid displacement of the ATMND from the aptamer. The released ATMND generates a high-intensity fluorescent signal, reporting the cocaine-binding event.

To develop a cocaine displacement-based sensor platform, we need a signal reporter which binds to the cocaine-binding aptamer but can also be displaced by cocaine, reporting the presence of the target. We recently discovered that the MNS-4.1 cocaine binding aptamer can also bind to the fluorophore ATMND and the cocaine binding can competitively displace ATMND from the aptamer-dye 
complex. Free ATMND is highly fluorescent in buffer, but ATMND is rapidly bound by MNS-4.1 upon addition of the aptamer, and we observed that ATMND fluorescence was greatly quenched within seconds. When we incubated $250 \mathrm{nM}$ ATMND with $2 \mu \mathrm{M}$ MNS-4.1, roughly $93 \%$ of the fluorescent signal was quenched, with the remaining $7 \%$ contributing to the low level of fluorescence background (Figure 4A). The aptamer-ATMND complex is very stable, and we did not observe any detectable fluorescence change over the course of 1 hour. We characterized sensor performance in terms of signal gain, which is the ratio of the backgroundsubtracted fluorescence obtained with cocaine relative to that obtained in the absence of cocaine, such that a larger signal gain is indicative of better sensitivity. The addition of cocaine is predicted to trigger a conformational rearrangement in the aptamer (Figure 3B), with two adjacent GA base pairs and a dinucleotide bulge (T20 and C21) within the aptamer binding pocket. ${ }^{62}$ Upon $50 \mu \mathrm{M}$ cocaine addition, we observed a competitive target binding to MNS-4.1, resulting in successful displacement of ATMND from the aptamer with a signal gain of 9.6 (Figure 4A). This fluorescence increase was stable for at least several hours (data not shown). We assume that this competitive binding results from a pure site-to-site displacement, although it could also be explained by a heterotropic allosteric effect. In contrast, we observed no measurable signal change in the absence of cocaine (Figure 4A). 
A

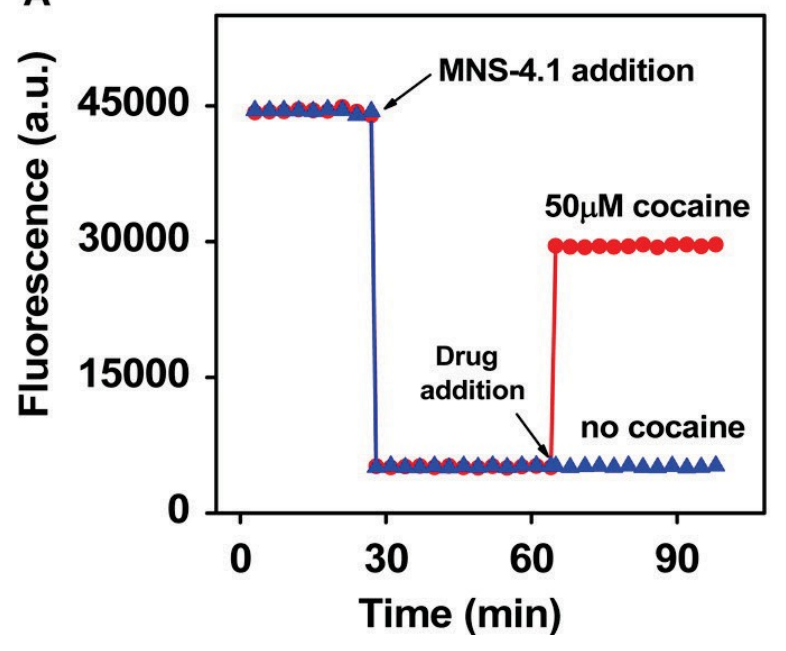

B

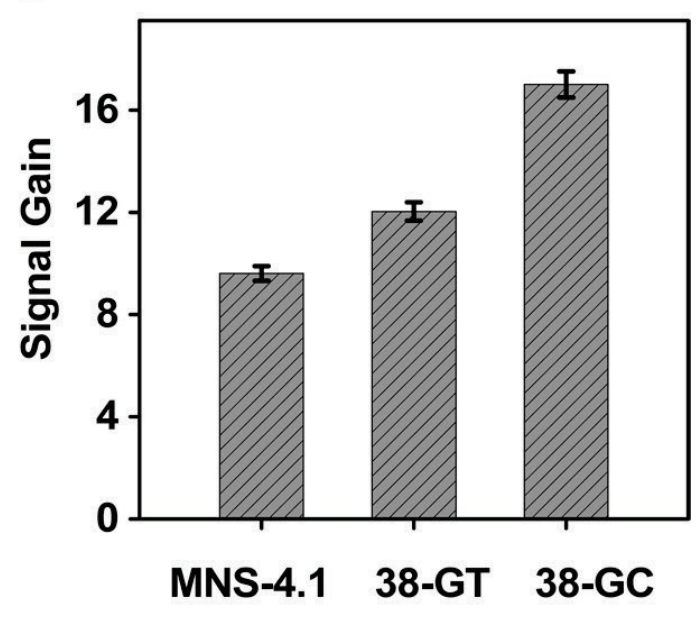

Figure 4. Our aptamer-based sensor rapidly responds in the presence of cocaine. (A) Time course of ATMND release. ATMND in buffer is highly fluorescent when excited at $358 \mathrm{~nm}$. ATMND is rapidly bound upon addition of the cocaine-binding aptamer, resulting in quenching of its fluorescence. In the presence of cocaine, the release of ATMND results in recovery of a strong fluorescent signal. (B) The impact of aptamer stability on signaling performance. The signal gain observed in the presence of cocaine was dependent on the composition of stem 1 and stem 3, which contributes to aptamer stability and ATMND/cocaine binding. Experimental conditions: $[\mathrm{DNA}]=2 \mu \mathrm{M},[$ ATMND $]=250 \mathrm{nM}$, [cocaine $]=50 \mu \mathrm{M}$. Error bars represent the standard deviation of three measurements.

Previous studies have suggested that the composition of stem 1 of the cocainebinding aptamer has the greatest impact on the secondary structure of the aptamer as well as the target binding affinity. ${ }^{62}$ MNS-4. 1 is only partially folded at equilibrium, ${ }^{60}$ and this is most likely due to the presence of three noncanonical base pairs in stem 1 , which would be expected to reduce the efficiency of ATMND binding and quenching and thereby result in high background fluorescence. Neves's group ${ }^{62}$ used 2D NMR spectral changes to confirm that the cocainebinding aptamer with six Watson-Crick base pairs in stem 1 is fully folded in the free form and exhibits increased binding affinity as compared to the original MNS 
4.1 aptamer. To test whether such a completely folded aptamer could reduce background fluorescence and boost signal gain, we engineered the 38-GT aptamer (Figure 3, 38-GT) by converting the three putative noncanonical base pairs in stem 1 to Watson-Crick base pairs, forming a seven-base-pair stem. Additionally, we tested whether decreasing the length of the stem would result in destabilization of the aptamer structure and studied the effects of such nucleotide deletions to the sensor performance. Interestingly, increasing the length of the stabilizing stem led to higher fluorescence quenching of a constant concentration of ATMND with little quenching being observed with an aptamer sequence with a short (3-base pair) stem 1 (Figure 5). These results suggest that binding of ATMND requires formation of the hydrophobic three-way junction pocket that can host the ATMND fluorophore, with increasing folding of the aptamer resulting in better binding of ATMND. The 38-GT (seven base-pairs in stem 1) resulted in reduced background fluorescence, with 95\% ATMND quenched (data not shown), indicating that ATMND binds tightly to 38-GT. We did not observe any loss of cocaine-displaced signal and obtained an increased signal gain of 12 in the presence of $50 \mu \mathrm{M}$ cocaine (Figure 4B). To further reduce the background signal, we subsequently converted the G-T wobble pair in stem 3 of $38-\mathrm{GT}$ to a matched G-C base pair (Figure 3, 38-GC). This increased structural stability led to a further increase of ATMND quenching efficiency (97\%) and an improved signal gain of 17 (Figure 4B) with $50 \mu \mathrm{M}$ cocaine. 


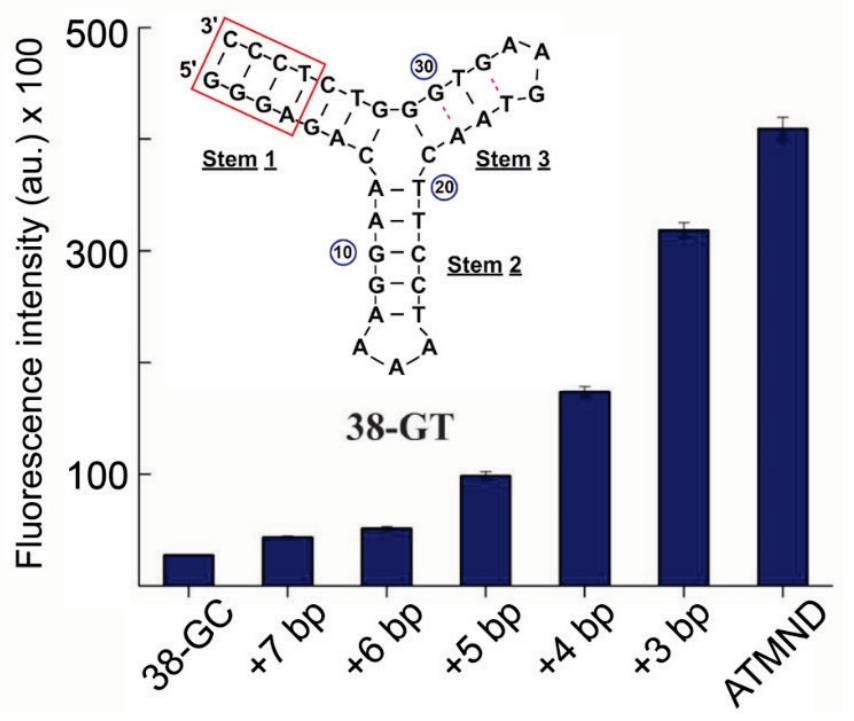

Figure 5. Stem-length effects on fluorophore binding and displacement by cocaine.

Various aptamer sequences were studied with increasing length of the structure stabilizing stem 1 from three base-pairs to seven base-pairs. Additionally, a seven base-pair sequence was further mutated by changing a non-match base-pair in stem 3 to a fully matched Watson-crick helix (termed 38-GC). Increasing the length of the stabilizing stem led to higher fluorescence quenching of a constant concentration of ATMND suggesting that formation of the three-way junction is necessary for binding of ATMND to the aptamer structure. The highest fluorescent quenching was observed with the 38-GC sequence which has the most stable and folded structure. [DNA] $=2 \mu \mathrm{M},[\mathrm{ATMND}]=250 \mathrm{nM}, \mathrm{Ex}: 358 \mathrm{~nm}$; Em: $405 \mathrm{~nm}$.

We presume that the greatly enhanced stability of 38-GC contributes to its high affinity toward both ligands, favoring formation of stable aptamer-ligand complexes and resulting in low background and high target-displaced signal gain. We used isothermal titration calorimetry (ITC) to investigate the binding affinity and thermodynamics of MNS-4.1, 38-GT, and 38-GC with regards to both ATMND and cocaine. The results confirmed that the aptamers bind both molecules, and that binding in both cases is enthalpically driven and entropically unfavorable (Table 1). ITC stoichiometry data indicated that each aptamer binds to one cocaine 
molecule or two ATMND molecules. After correction of the dilution heat, we used the single-site binding model to obtain equilibrium dissociation constants $\left(K_{\mathrm{dc}}\right)$ of $6.7 \pm 1.3 \mu \mathrm{M}$ (Figure $6 \mathrm{~A}$ ), $10 \pm 1 \mu \mathrm{M}$ (Figure $6 \mathrm{C}$ ) for cocaine binding to MNS-4.1 and 38-GT, respectively. Interestingly, affinity for cocaine is essentially unchanged even after stabilization of the aptamer structure with completely matched helix in stem 1. We used the two sets of sites binding model to obtain affinity measurements for ATMND binding sites on MNS-4.1 $\left(K_{\mathrm{d} 1}, 6.6 \pm 0.8 \mu \mathrm{M} ; K_{\mathrm{d} 2}, 25 \pm\right.$ $1 \mu \mathrm{M})$ (Figure 6B) and 38-GT (Kd1, $\left.0.16 \pm 0.08 \mu \mathrm{M} ; K_{\mathrm{d} 2}, 6.3 \pm 1.2 \mu \mathrm{M}\right)$ (Figure 6D). In contrast to cocaine binding, ATMND affinity increases substantially after substitution of the mismatched base-pairs in stem 1 with a completely matched helix. Accordingly, the higher levels of fluorescence quenching are observed presumably because of the higher affinity of ATMND to the more stable fully matched helix as observed from the fluorescence experiments. ITC data also showed that the $\mathrm{G}-\mathrm{C}$ change in $38-\mathrm{GC}$ further increases the stability of the aptamer, leading to increased binding affinity for both ligands. Compared with 38GT, we observed 4-fold enhanced binding to cocaine $\left(K_{\mathrm{dc}}=2.6 \pm 1.0 \mu \mathrm{M}\right)$ and 10 fold tighter binding to ATMND $\left(K_{\mathrm{d} 1}=0.016 \pm 0.001 \mu \mathrm{M} ; K_{\mathrm{d} 2}=2.6 \pm 1.0 \mu \mathrm{M}\right)($ Figure 7). The thermodynamics of binding indicate that the binding of both molecules is highly enthalpically driven. This suggests strong hydrogen bonding interactions upon binding of both molecules. Interestingly, cocaine binding results in higher levels of enthalpy change. This is presumably because cocaine binding can induce changes in the aptamer conformation, resulting in creation of new hydrogen bonds. Unlike cocaine, ATMND does not affect the structure of the aptamer, but rather 
might just reside in the 3-way junction pocket of the host aptamer and hydrogen bond with unpaired nucleotides in the absence of cocaine.

Table 1. Binding and thermodynamic parameters of 38-GT and 38-GC for ATMND and cocaine*

\begin{tabular}{|c|c|c|c|c|}
\hline Ligand & $\mathbf{K}_{d}(\mu \mathrm{M})$ & $\mathbf{N}$ & $\Delta \mathrm{H}\left(\mathrm{kcal} \mathrm{mol}^{-1}\right)$ & $-\mathrm{T} \Delta \mathrm{S}\left(\mathrm{kcal} \mathrm{mol}^{-1}\right)$ \\
\hline \multirow{2}{*}{ ATMND-MNS-4.1 } & $6.6 \pm 0.8$ & 1 & $-27.5 \pm 0.8$ & 20 \\
\hline & $25 \pm 1$ & 1 & $-54.1 \pm 0.1$ & 48 \\
\hline Cocaine-MNS-4.1 & $6.7 \pm 1.3$ & 1 & $-60 \pm 3$ & 53 \\
\hline \multirow[t]{2}{*}{ ATMND-38-GT } & $0.16 \pm 0.08$ & 1 & $-36.1 \pm 0.2$ & 27 \\
\hline & $6.3 \pm 1.2$ & 1 & $-49.8 \pm 0.9$ & 43 \\
\hline Cocaine-38-GT & $10 \pm 1$ & 1 & $-68.2 \pm 1.1$ & 61 \\
\hline \multirow[t]{2}{*}{ ATMND-38-GC } & $0.016 \pm 0.001$ & 1 & $-14.2 \pm 0.2$ & 3.6 \\
\hline & $2.6 \pm 1.0$ & 1 & $-16.9 \pm 0.3$ & 9.2 \\
\hline Cocaine-38-GC & $2.6 \pm 1.0$ & 1 & $-24.5 \pm 0.3$ & 17 \\
\hline
\end{tabular}



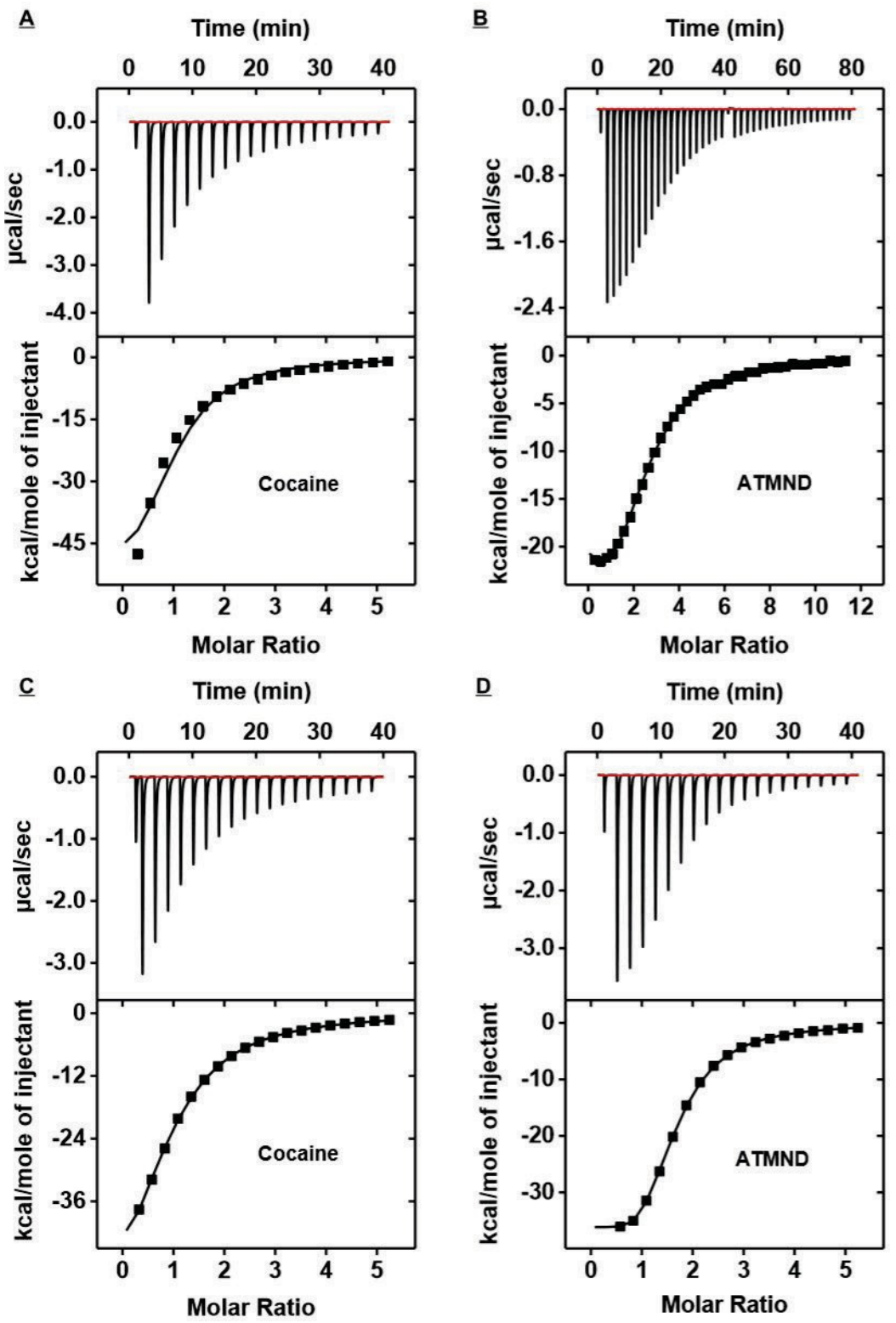

Figure 6. ITC data demonstrated that MNS-4.1 and 38-GT binds both cocaine and ATMND.

ITC data showing heat generated from each injection of $(A)$ cocaine or (B) ATMND into the MNS-4.1 aptamer solution. (C, D) ITC data showing heat generated from each injection and integrated heat plot of (C) cocaine or (D) ATMND into the 38GT aptamer solution. Experimental conditions: [MNS-4.1 or 38-GT] $=20 \mu \mathrm{M}$, [ATMND] $=500 \mu \mathrm{M}$ and [cocaine] $=500 \mu \mathrm{M}$. Binding experiments were performed in $10 \mathrm{mM}$ Tris $(\mathrm{pH} 7.4)$ including $0.01 \mathrm{mM} \mathrm{MgCl}_{2}$ and $5 \% \mathrm{DMSO}$ at $25^{\circ} \mathrm{C}$. 
A

Time (min)

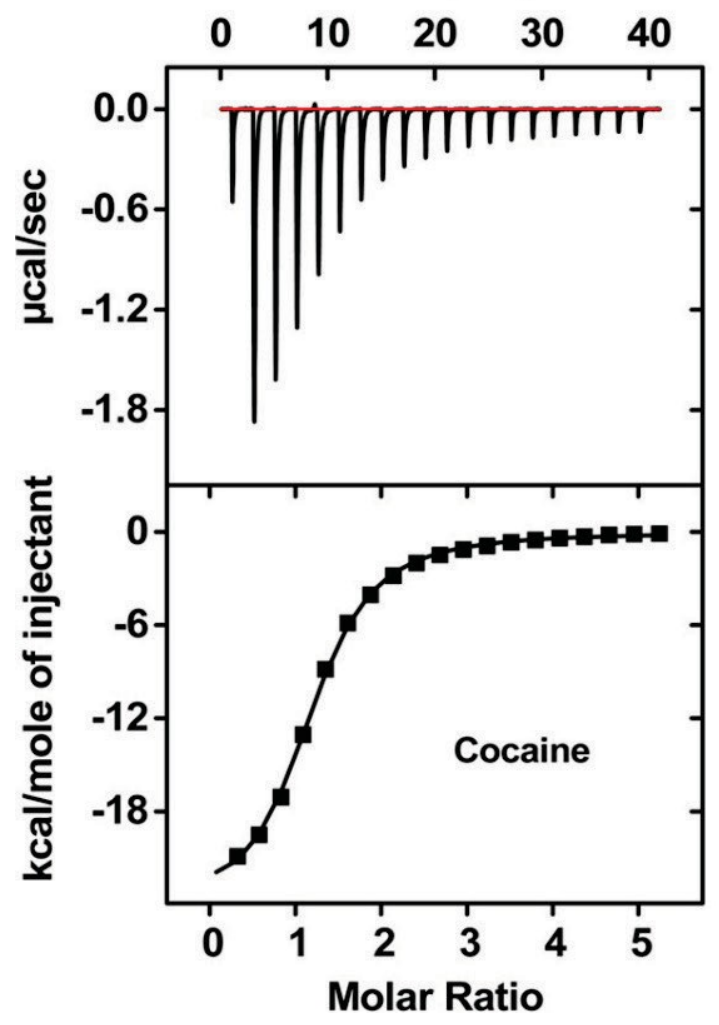

B Time (min)

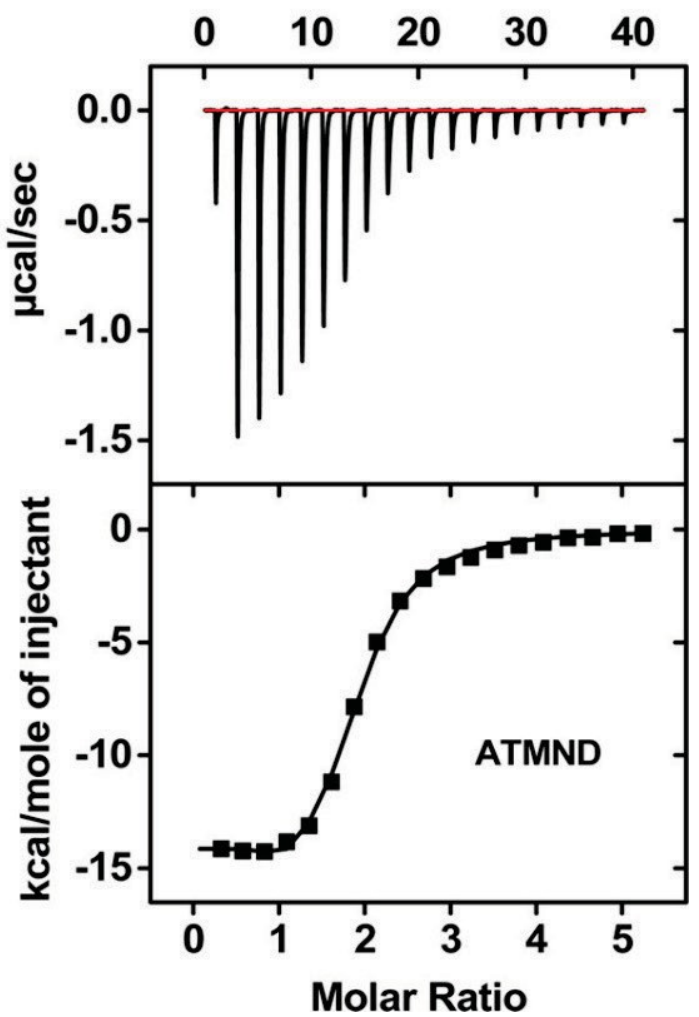

Figure 7. 38-GC binds both cocaine and ATMND.

(Top) ITC data showing heat generated from each injection of (A) cocaine or (B) ATMND into the 38-GC solution. (Bottom) The integrated heat plot after correcting for the heat of dilution. Experimental conditions: $[38-G C]=20 \mu \mathrm{M},[$ ATMND] $=500$ $\mu \mathrm{M}$, and [cocaine] $=500 \mu \mathrm{M}$.

These results demonstrate the potential of ATMND as an excellent transduction element; its tight binding to the aptamer results in a very low background signal until it becomes displaced via competitive binding of cocaine, with incredibly fast recovery of its fluorescence (i.e., seconds). To minimize the background signal and obtain a high signal-to-noise ratio, we used a fixed concentration of 38-GC $(2 \mu \mathrm{M})$ to optimize the concentration of ATMND and thus obtained an optimized 38GC:ATMND ratio of 8:1 (Figure 8); under these conditions, we calculated that 99.3\% dye-aptamer complexes contained only one ATMND molecule bound at 
the strong binding site, while the concentration of complexes containing two ATMND molecules was sufficiently low as to be negligible (see modeling calculations).

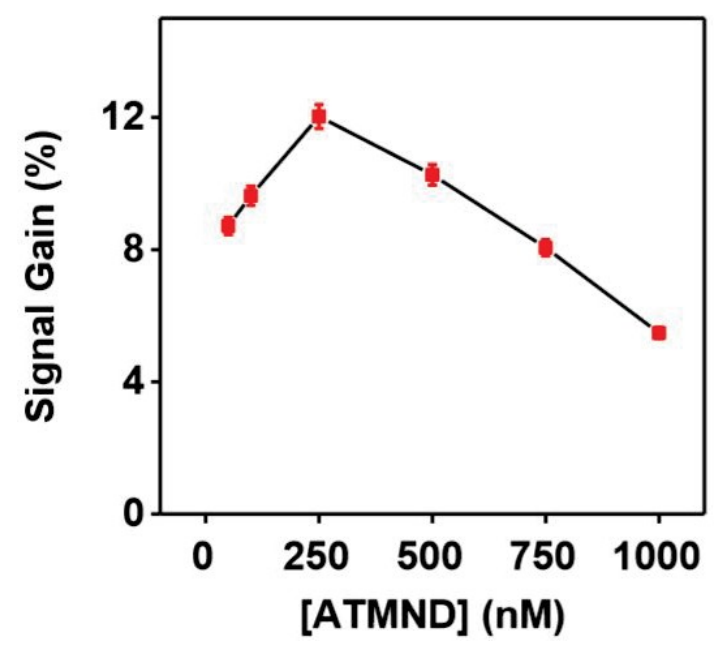

Figure 8. Effect of different concentrations of ATMND on fluorescence signal gain. Maximum signal gain was achieved at an optimized molar ratio between the 38GC aptamer and ATMND of 8:1. [38-GC] $=2 \mu \mathrm{M}$, [cocaine] $=50 \mu \mathrm{M}$, with excitation at $358 \mathrm{~nm}$ and emission at $405 \mathrm{~nm}$.

\subsubsection{Modeling data for binding of ATMND to 38-GC:}

Two assumptions are required for the calculation

1. One DNA aptamer molecule can bind one or two ATMND molecules in its hydrophobic pocket;

2. Two ATMND molecules can occupy the hydrophobic binding pocket independently

In the presence of ATMND, a DNA aptamer molecule can bind a single ATMND molecule strongly at site 1 to form complex 1 or it can bind one ATMND molecule weakly at site 2 to form complex 2 , establishing two equilibriums in the solution: 
Site 1 (strong binding):

$$
\mathrm{DNA}+\mathrm{ATMND} \underset{\mathrm{K}_{\mathrm{d} 1}}{\stackrel{1 / \mathrm{K}_{\mathrm{d} 1}}{\rightleftharpoons}} \text { Complex } 1
$$

Site 2 (weak binding)

$$
\mathrm{DNA}+\mathrm{ATMND} \underset{\mathrm{K}_{\mathrm{d} 2}}{\stackrel{1 / \mathrm{K}_{\mathrm{d} 2}}{\rightleftharpoons}} \text { Complex } 2
$$

There is also a possibility for complex 1 to bind a second ATMND molecule weakly or for complex 2 to bind a second ATMND molecule strongly. Thus, another two related equilibriums are formed independently:

$$
\begin{aligned}
& \text { Complex 1 + ATMND } \underset{\mathrm{K}_{\mathrm{d} 2}}{\stackrel{1 / \mathrm{K}_{\mathrm{d} 2}}{\rightleftharpoons}} \text { Complex } 3 \\
& \text { Complex 2+ ATMND } \stackrel{1 / \mathrm{K}_{\mathrm{d} 1}}{\underset{\mathrm{K}_{\mathrm{d} 1}}{\rightleftharpoons}} \text { Complex } 3
\end{aligned}
$$

( $K_{\mathrm{d} 1}$ and $\mathrm{K}_{\mathrm{d} 2}$ stand for the dissociation constant between the ATMND molecule and the DNA aptamer at its strong binding site or its weak-binding site, respectively.) The dissociation constant for reactions (I-IV) can then be defined in terms of the equilibrium concentration of each component: 


$$
\begin{aligned}
& K K_{d d 1}=\frac{[D D D D D D][D D A A A A D D D D]}{[C C C C C C C C C C C C \text { 1] }} \\
& K_{\mathrm{dd2}}=\frac{[\mathrm{DDDDDD}][\mathrm{DDAAAADDDD}]}{[C C C C C C C C C C C C 2]} \\
& K K_{d d 2}=\frac{[\text { CCCCCCCCCCCCC } 1][\text { DDAAAADDDD }]}{[\operatorname{CCCCCCCCCCCCC~3]}}
\end{aligned}
$$

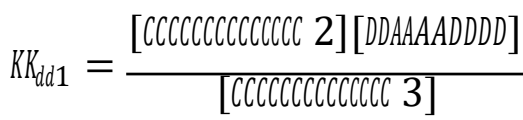

By using equation (1) to divide equation (2), we deduce equation (5):

$$
\frac{[\operatorname{ccccccccccccc} 2]}{[\operatorname{ccccccccccccc} 1]}=\frac{K K_{d d 1}}{K K_{d d 2}}
$$

Equation (6) can be deduced from equations (2) and (3),

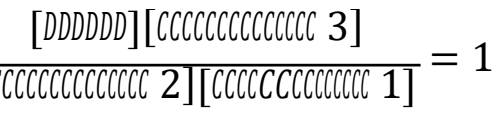

Thus,

$$
[\operatorname{cccccccccccccc} 3]=[\operatorname{ccccccccccccccc} 2] \frac{[\operatorname{ccccccccccccccc} 1]}{[\mathrm{DDDDD}]}
$$

ATMND is not completely consumed in the reaction due to the equilibrium; therefore a comparison can be successfully made between the equilibrium concentration of ATMND and DNA and their total concentration (denoted as ATMNDt and DNAT) 


$$
\frac{[C C C C C C C C C C C C}{1]}<\frac{D_{A A A A D D D D_{T T}}}{[D D D D D D]}=\frac{0.25 u A A}{\text { DDDDDD }_{T \Gamma}-D_{D A A A A D D D_{T T}}}=\frac{1}{7}
$$

Equation (6) can be recalculated using the solution from equation (7) to obtain the comparison of the equilibrium concentration between complex 2 and complex 3 :

$$
[\operatorname{cccccccccccccc} 3]<\frac{1}{7}[\text { [ccccccccccccc 2] }
$$

Based on ITC data, the values of dissociation constants ( $K_{d 1}$ and $\left.K_{d 2}\right)$ of ATMND to $38-\mathrm{GC}$ are $0.016 \mathrm{uM}$ and $2.6 \mathrm{uM}$, respectively. Thus, a relationship between the concentration of the three complexes is established as follows:

$$
[\text { [ccccccccccccc 1] }=162.5[\operatorname{cccccccccccccc} 2]>1137.5[\operatorname{cccccccccccccc} 3]
$$

Thus, the percentage of complex 1 :

$$
\begin{aligned}
& \frac{[\operatorname{cccccccccccccc} 1]}{[\operatorname{cccccccccccccc} 1]+[\operatorname{ccccccccccccc} 2]+[\operatorname{cccccccccccccc} 3]} \\
& =\frac{1}{[\operatorname{ceccccccccccc} 1]}+\frac{1}{[\operatorname{ceccccccccccc} 1]}+\frac{1}{[\operatorname{ceccccccccccc} 1]}+\frac{1}{1+\frac{1}{162.5}+\frac{1}{1187.5}} \\
& =99.3 \%
\end{aligned}
$$

Since $99.3 \%$ of the ATMND-aptamer complex is complex 1 in which the aptamer only bind one ATMND at the strong site, we have ignored the formation of complex and complex 3 in the reaction. 


\subsubsection{Mutation of binding pocket nucleotides}

Our sensor is modeled on the understanding that both ATMND and cocaine compete for the same aptamer, and we assume that the binding site of both ligands is located within the hydrophobic three-way junction pocket. To confirm this assumption, we examined the extent to which targeted nucleotide changes affects ligand binding and target competition. We designed mutants of 38-GC in which we introduced a nucleotide switch between T20 and C21 (38-GC-M1; Figure 9) or a replacement of thymine at position 20 with an adenine (38-GC-M2; Figure 9). Previous publications have determined that such mutations eliminate binding affinity for cocaine. We tested these mutant aptamers in our competitive cocainebinding fluorescence assay and observed significantly reduced binding to both ATMND and cocaine, with a high fluorescence background and a low cocainedisplaced fluorescence recovery (Figure 9). This poor ATMND binding and weak cocaine displacement resulted in a signal gain of just 1.3 and 1.2 with $50 \mu \mathrm{M}$ cocaine for 38-GC-M1 and 38-GC-M2, respectively. This very weak binding affinity of mutant aptamers to both ATMND and cocaine was also confirmed by ITC (Figure 10). The enthalpy change in the binding interaction of both ATMND and cocaine with the mutated aptamers was much smaller, suggesting inhibition of hydrogen bonding. This is consistent with previous studies showing that mutations to nucleotides that contribute to formation of this hydrophobic pocket, including the unpaired T20 and C21, can profoundly alter the aptamer's binding affinity for cocaine..$^{149}$ 
Our mutation experiments indicate that ATMND binding is also heavily dependent on C21 and T20. Nakatani et al. ${ }^{150,151}$ used ${ }^{15} \mathrm{~N}$ NMR experiments to reveal that 1,8-naphthridine selectively binds to cytosine or thymine via a three-point hydrogen bond ${ }^{152}$ and that the binding affinity of ATMND to various target nucleotides is as follows: cytosine $>$ thymine $>$ adenine $>$ guanine.$^{66}$ Thus, it is likely that ATMND binds either C21 or T20 within the three-way junction of the aptamer through a three-point hydrogen bond. ${ }^{153,154}$ Additionally, we observed a large negative enthalpy change in ITC experiments, which also indicates the formation of hydrogen bonds between ATMND and the aptamer. We believe that ATMND forms these hydrogen bonds and possibly cooperates with adjacent nucleotides via mstacking interaction to significantly quench its fluorescence (figure 11). ${ }^{153-156}$ 


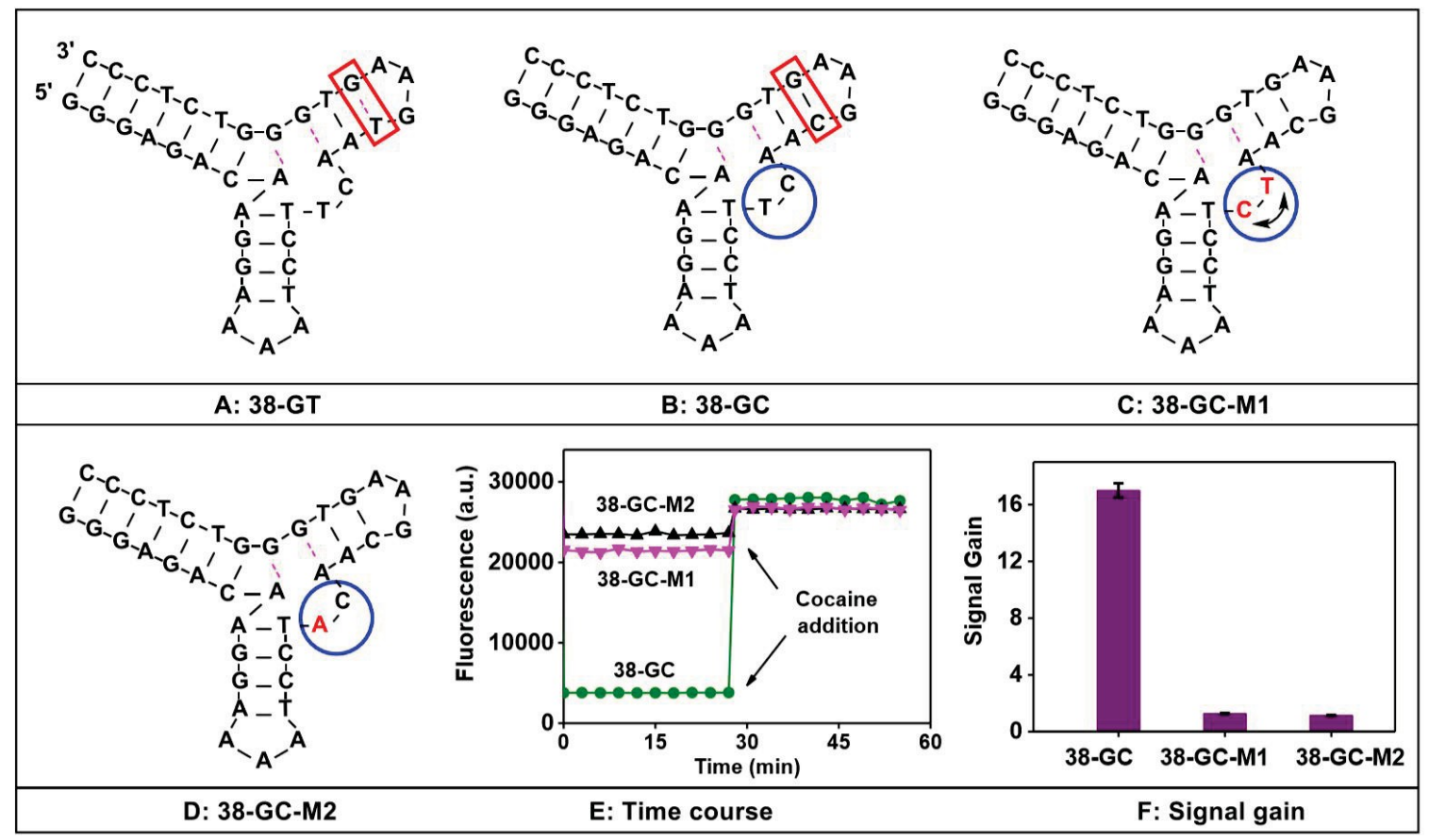

Figure 9. (A-D) Modifications of the 38-GT aptamer. Convert a GT base-pairing to a GC base-pairing in stem 3 (B) further increases its ligand binding affinity, whereas mutations in the binding pocket $(C, D)$ reduce ligand affinity. $(E)$ Mutations to the unpaired bases in the hydrophobic binding pocket of 38-GC alter ATMND binding and cocaine detection. Both the 38GC-M1 and 38-GC-M2 mutants exhibited decreased quenching efficiency and cocaine-displaced signal gain. (F) A fluorescence binding assay shows the differences in signal gain for these various 38-GT derivatives in the presence of $50 \mu \mathrm{M}$ cocaine. Experimental conditions: $[\mathrm{DNA}]=2 \mu \mathrm{M},[\mathrm{ATMND}]=250 \mathrm{nM}$. Error bars represent the standard deviation of three measurements. 
$\underline{A}$

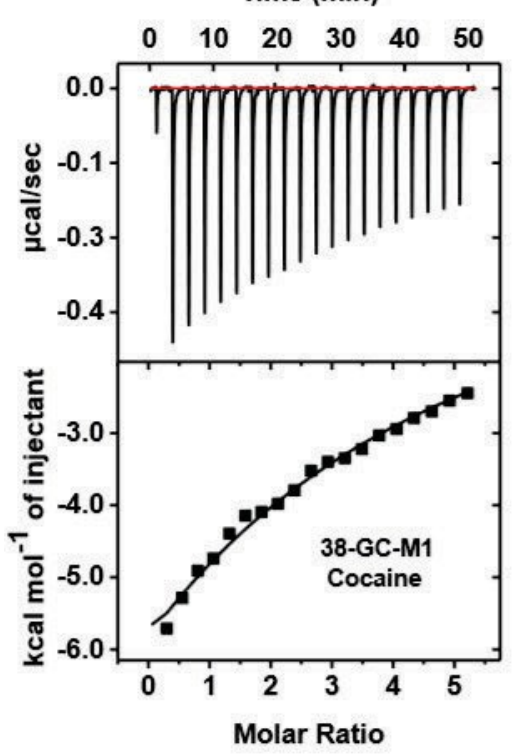

$\underline{\mathbf{c}}$

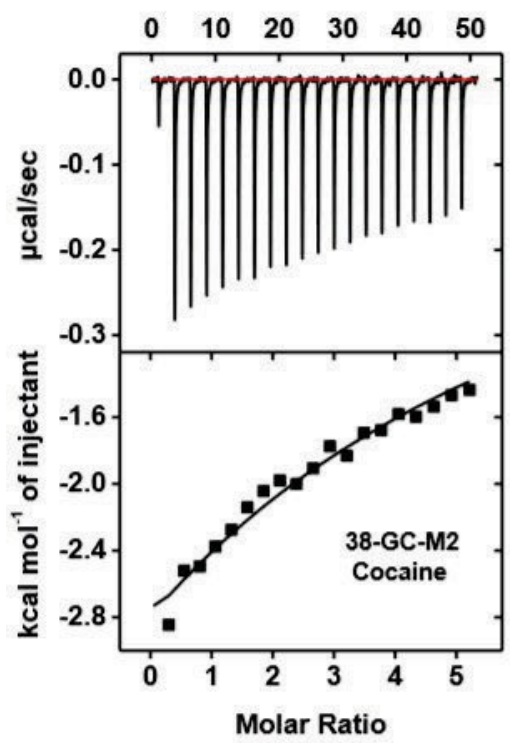

B $\quad$ Time (min)

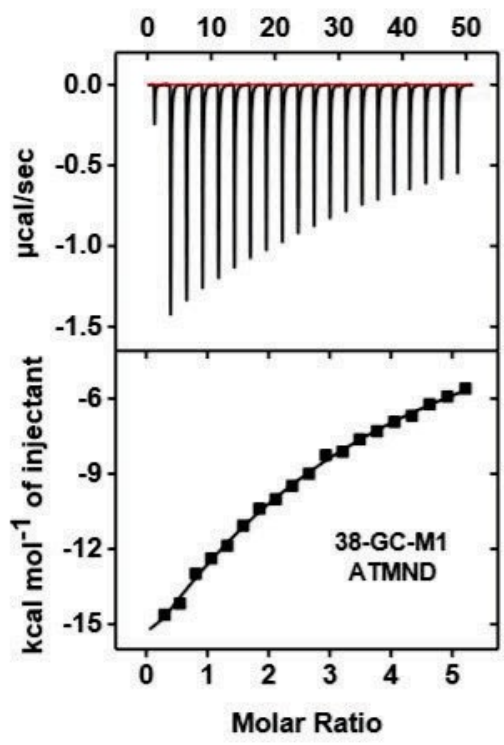

$\underline{\mathbf{D}}$

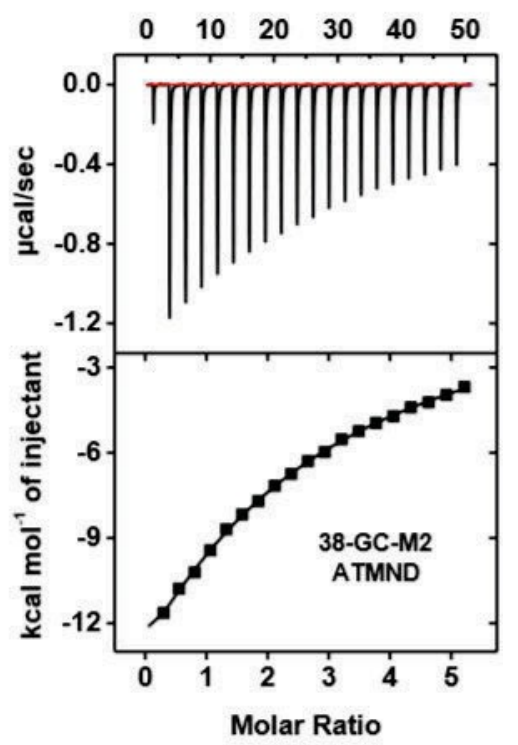

Figure 10. 38-GC-M1 and 38-GC-M2 demonstrated a very weak binding affinity for ATMND and cocaine.

(A, B) ITC data showing heat generated from each injection and integrated heat plot of (A) cocaine or (B) ATMND into the 38-GC-M1 aptamer solution. (C, D) ITC data showing heat generated from each injection and integrated heat plot of $(C)$ cocaine or (D) ATMND into the 38-GC-M2 aptamer solution. Experimental conditions: [38-GC-M1/M2] $=20 \mu \mathrm{M}$, [ATMND] $=500 \mu \mathrm{M}$ and [cocaine] $=500 \mu \mathrm{M}$. Binding experiments were performed in $10 \mathrm{mM}$ Tris ( $\mathrm{pH}$ 7.4) including $0.01 \mathrm{mM}$ $\mathrm{MgCl} 2$ and $5 \%$ DMSO at $25^{\circ} \mathrm{C}$. 
<smiles>Cc1nc2nc(N)ccc2c(C)c1C</smiles>

Fluorescence
MNS-4.1

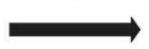

Figure 11. Binding of ATMND to the cocaine aptamer.

Mutational studies and isothermal titration calorimetry indicate that formation of the three-way junction structure and the cytosine at position 20 are highly important for binding of ATMND. Thus, we propose that ATMND possibly binds at this site thru hydrogen bonding as it can be protonated in solution. ITC experiments exhibit large enthalpy changes which suggest formation of such hydrogen bonds. Binding of ATMND is essentially eliminated upon mutation of the C20 nucleotide, suggesting a possible binding site for ATMND.

Our sensor is based on a simple, one-pot, one-step reaction that entails the simple mixing of 38-GC, ATMND, and cocaine at room temperature and then exploiting the rapid competition between cocaine and ATMND for limited aptamer binding sites to quantitatively detect cocaine in samples. We observed that the signal gain increased with cocaine concentration, reaching a saturated signal gain of 19 with $250 \mu \mathrm{M}$ cocaine (Figure 12A), with a linear range of $0-8 \mu \mathrm{M}$ (Figure 12A, inset). The low background resulting from the high affinity interaction of 38-GC with ATMND yields an excellent limit of detection (LOD). In a reaction with $2 \mu \mathrm{M} 38-\mathrm{GC}$ and $250 \mathrm{nM}$ ATMND, we achieved an LOD of $200 \mathrm{nM}$ within $20 \mathrm{~s}$, more than 50fold better than most existing aptamer-based systems $59,63,64,142,148,157$ and comparable to sensitive assays that require enzymatic amplification (Table 2). The LOD was calculated as $S / N>3$, where the noise was a drug-free sample and the minimum amount of sample was 3 times this amount. 

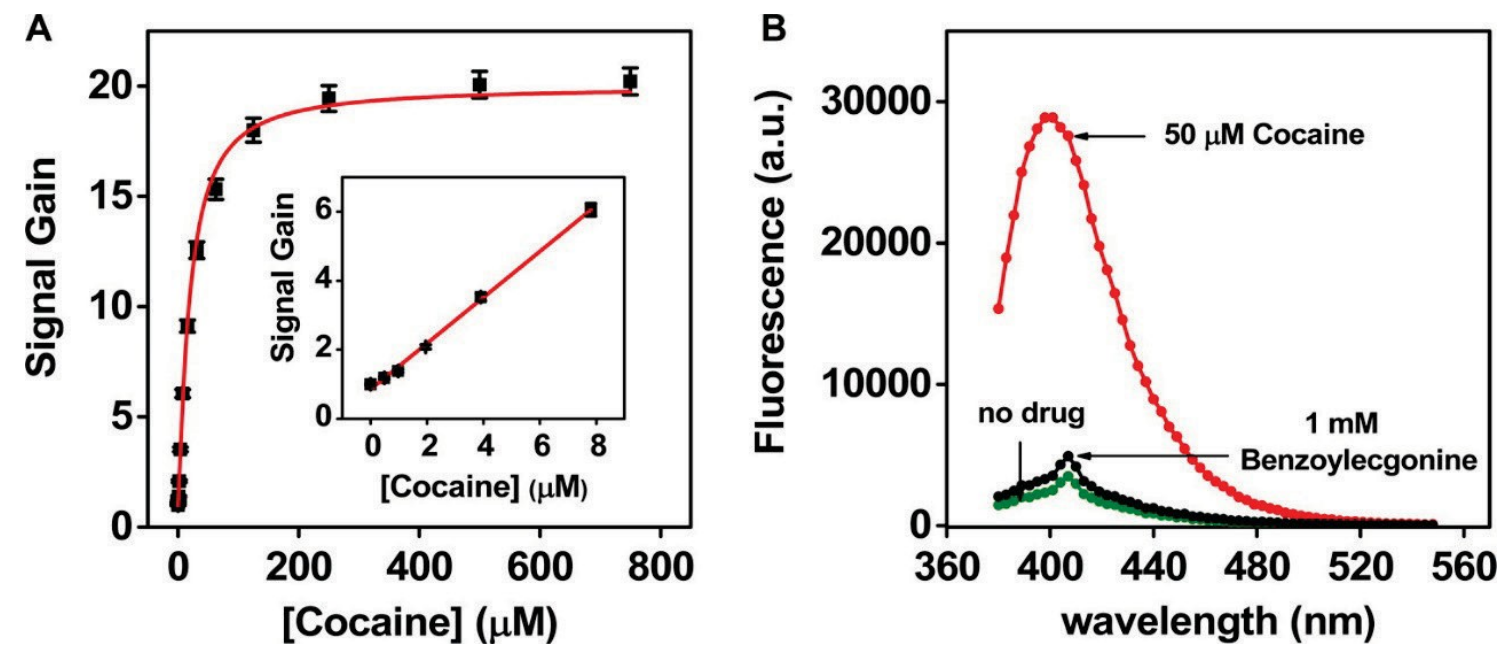

Figure 12. Sensitivity and specificity of the ATMND-based sensor in the reaction buffer.

(A) Calibration curve shows a strong concentration-dependent response to cocaine by 38-GC-ATMND. (B) Our sensor specifically responds to cocaine but exhibits almost no response to its major metabolite benzoylecgonine. Experimental conditions: $[\mathrm{DNA}]=2 \mu \mathrm{M},[\mathrm{ATMND}]=250 \mathrm{nM}$. Error bars in plot $\mathrm{A}$ represent the standard deviation of three measurements conducted at each cocaine concentration.

Table 2. Comparison of aptamer-based sensors for cocaine detection

\begin{tabular}{|c|c|c|}
\hline Method & Limit of detection & Response time \\
\hline Cy7 displacement ${ }^{1}$, Colorimetric & $2 \mu \mathrm{M}$ & 12 hours \\
\hline SYBR-gold binding ${ }^{2}$, Fluorescence & $5 \mu \mathrm{M}$ & 3 hours \\
\hline Strand-displacement amplification ${ }^{3}$, Fluorescence & $2 \mathrm{nM}$ & $>2$ hours \\
\hline Aptamer self-assembly ${ }^{4}$, Fluorescence & $10 \mu \mathrm{M}$ & $1-2$ hours \\
\hline DNAzyme-amplified detection ${ }^{5}$, Colorimetric & $50 \mathrm{nM}$ & $40 \mathrm{~min}$ \\
\hline Aptamer conformational change ${ }^{6}$, Fluorescence & $2 \mu \mathrm{M}$ & Minutes \\
\hline Modified gold-nanoparticle based ${ }^{7}$, Colorimetric & $50 \mu \mathrm{M}$ & $1 \mathrm{~min}$ \\
\hline Microcantilever ${ }^{8}$, Interferometric & $5 \mu \mathrm{M}$ & $25 \mathrm{~min}$ \\
\hline Aptamer conformational change ${ }^{9}$, Electrochemistry & $10 \mu \mathrm{M}$ & Seconds \\
\hline 38-GC-ATMND complex, Fluorescence (this work) & $200 \mathrm{nM}$ & Seconds \\
\hline
\end{tabular}

The 38-GC-ATMND complex offers a superior signal transduction mechanism for immediate and specific cocaine detection with a robust signal gain. Since Cy7 has 
been used as a signal reporter in a previously reported cocaine-mediated aptamerbased assay, ${ }^{60}$ we compared the sensor performance of 38-GC with either Cy7 or ATMND under optimized conditions and confirmed that 38-GC exhibits a much better signal gain and sensitivity with ATMND. For example, our ATMND-based "signal-on" sensor produces an approximate signal gain of 17 with only $1.5 \%$ of relative standard deviation (RSD) in the presence of $50 \mu \mathrm{M}$ cocaine (Figure 12A), while the same cocaine concentration produces only a $35 \%$ signal decrease with $5 \%$ of RSD for the Cy7-based "signal-off" sensor (Figure 13).

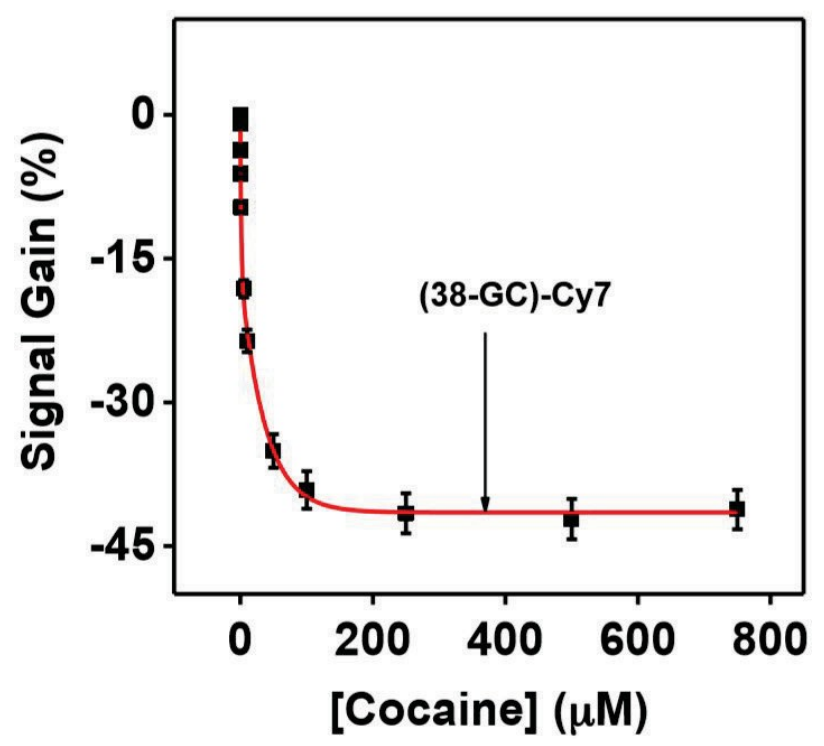

Figure 13. Calibration curve for a 38-GC-Cy7-based sensor.

Experimental conditions: [38-GC] $=2 \mu \mathrm{M},[\mathrm{Cy} 7]=7 \mu \mathrm{M}$. Error bars represent the standard deviation of three measurements conducted at each cocaine concentration. Relative sensor response in terms of percent change is shown, because this is more reproducible than absolute absorbance values. The signal gain is calculated as [(Abscocaine-cy7 - Absbackground) / (Abscy 7 - Absbackground) $) \times 100 \%$ where the background is derived from buffer and absorbance was measured at $760 \mathrm{~nm}$. 
Cocaine-binding aptamers generally exhibit good specificity in terms of distinguishing cocaine from structurally similar major metabolites. ${ }^{57} \mathrm{We}$ tested the specificity of our assay with benzoylecgonine, which is the corresponding carboxylic acid derivative of cocaine with an otherwise almost identical structure. ${ }^{158,159}$ Relative to the signal gain of 17 obtained with $50 \mu \mathrm{M}$ cocaine, we did not detect a measurable signal change upon addition of $50 \mu \mathrm{M}$ benzoylecgonine and observed a signal gain of just 1.7 when we increased the benzoylecgonine concentration to $1 \mathrm{mM}$ (Figure 12B), which is consistent with the good specificity observed with previous aptamer-based sensors (Table 2). Additionally, we tested several other compounds for cross-reactivity with our sensing method, including cocaethylene (biomarker of alcohol and cocaine consumption), diphenhydramine (allergy medicine and cutting agent), cocaine metabolites and cutting agents in street drugs. Several of these compounds do not result in any measurable signal increase, however we did notice some crossreactivity with the cocaine metabolite cocaethylene and the drugs diphenhydramine and promazine (Figure 14). Cross-reactivity with cocaethylene would allow for detection of co-consumption of alcohol and cocaine while crossreactivity with over the counter drugs such as diphenhydramine might be problematic in instances where they are being consumed as medicine or are present as cutting agents in street samples. Wang et al. detailed a method that can be used to increase the specificity for the same cross-reactive molecules by utilizing an enzyme-based detection method. ${ }^{110}$ 

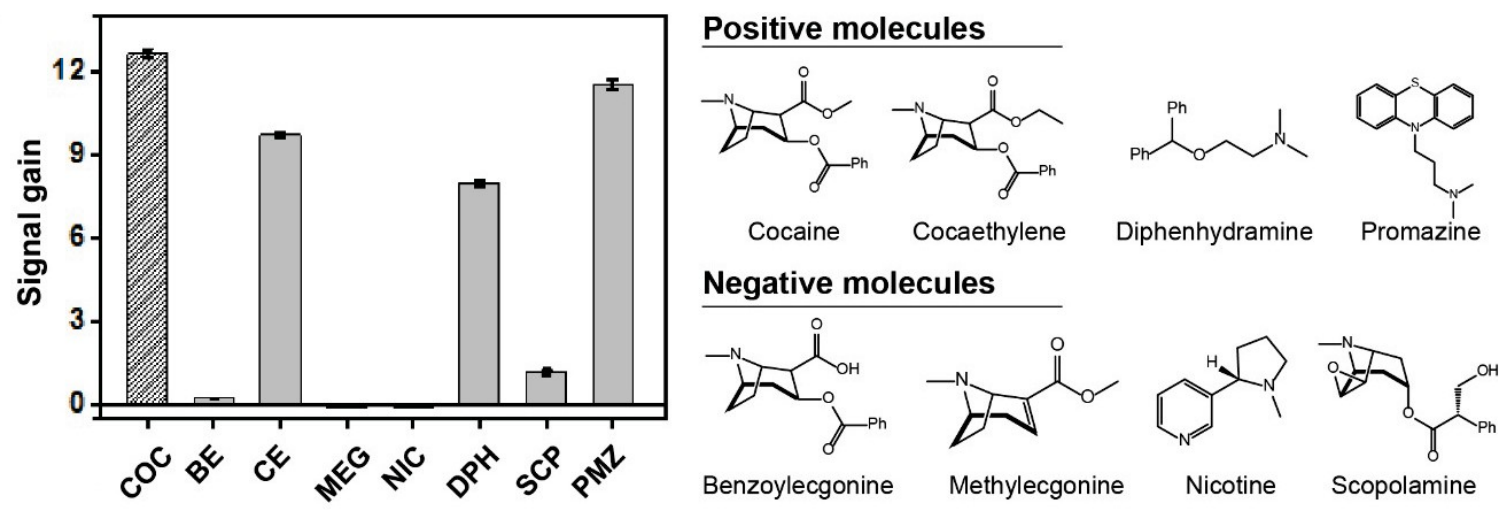

Figure 14. Cross-reactivity of the ATMND-sensor for several compounds of interest in cocaine measurements.

Cocaethyelene metabolite was found to bind to the aptamer and result in increased fluorescence as well as diphenhydramine (allergy medicine) and promazine (antipsychotic). The cutting agent scopolamine does not result in any interference.

The average concentration of cocaine in different body fluids within $24 \mathrm{~h}$ after ingestion is typically greatest in the urine (milligrams per liter), with lower concentrations found in saliva and serum, respectively. ${ }^{56}$ We explored the practicality of our assay for performing cocaine detection in different body fluids collected from healthy donors. We first tested our assay with $250 \mu \mathrm{M}$ cocaine spiked into various dilutions of urine, saliva, or serum (Figure 15) samples. We observed that the signal gain increased in inverse proportion to the biofluid concentration, with a maximum signal gain of $8.2,9.6$, and 5.6 for $2.5 \%$ urine, saliva, and serum, respectively. Human urine normally contains very small amounts of protein $(<0.14 \mathrm{mg} / \mathrm{mL})^{160}$ and a large number of fluorescent metabolites. ${ }^{161,162}$ We observed that urine samples indeed generated a strong background fluorescence at 400-550 nm (Figures 16 and 17) and metabolites such as pterins, flavins, porphyrins, and 4-pyridoxic acid may be contributing to this fluorescence. ${ }^{163}$ Although the urine matrix exhibited a high background 
fluorescence, we still achieved successful detection of cocaine. We observed that the signal gain increased with increasing cocaine concentrations, with an LOD of $460 \mathrm{nM}$ cocaine in $2.5 \%$ urine (Figure $18 \mathrm{~A}$ ).
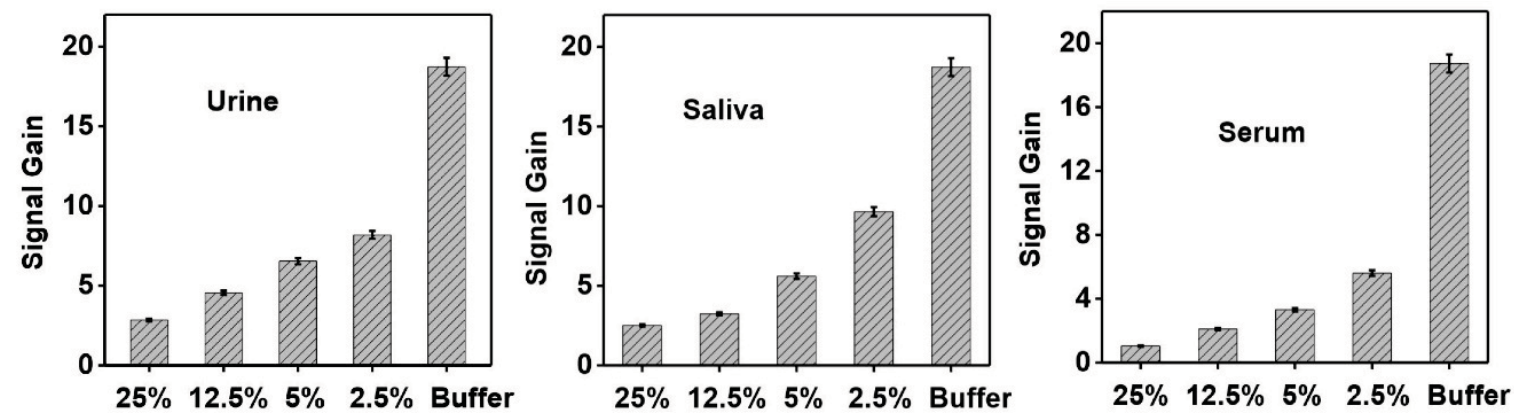

Figure 15. Successful detection of cocaine spiked into different dilutions of urine, saliva and serum with 38-GCATMND.

Signal gain decreases with increasing concentrations of urine. Experimental conditions: $[38-\mathrm{GC}]=2 \mu \mathrm{M},[$ ATMND] $=250 \mathrm{nM}$ and [cocaine] $=250 \mu \mathrm{M}$, with excitation at $358 \mathrm{~nm}$ and emission at $405 \mathrm{~nm}$. Error bars represent the standard deviation of three measurements. The signal gain is calculated as ( $F_{\text {cocaine-ATMND - }}$ $F_{\text {background }}$ / ( $\left.F_{\text {ATMND }}-F_{\text {background }}\right)$ where the background is derived from the biofluid sample.

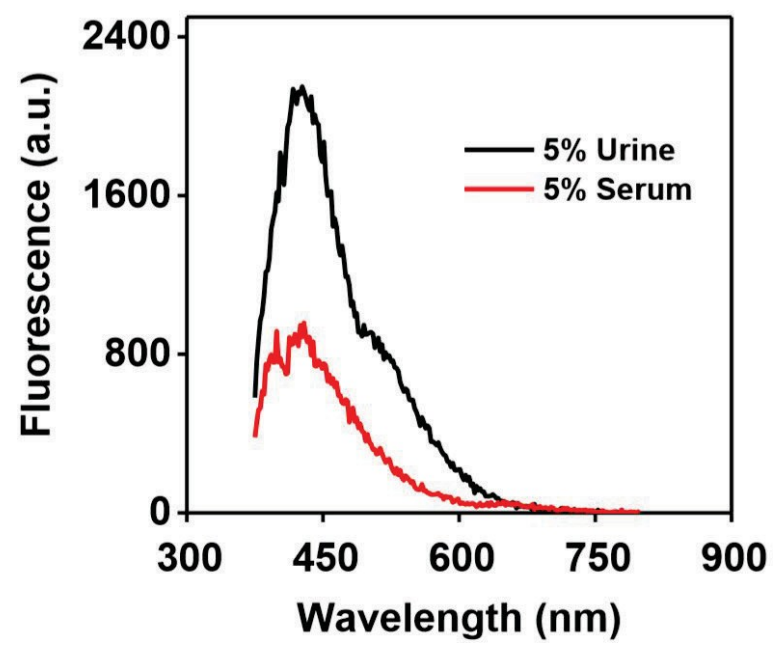

Figure 16. 5\% urine and serum samples emit fluorescence within the wavelength range from $375 \mathrm{~nm}$ to $600 \mathrm{~nm}$ when excited at $358 \mathrm{~nm}$. 


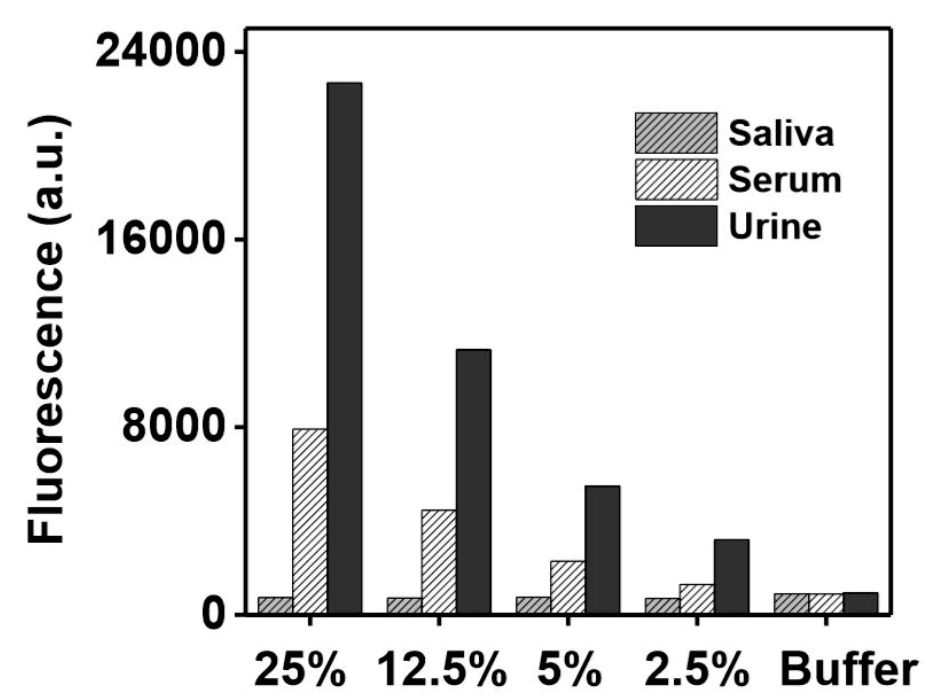

Figure 17. Fluorescence intensities for different concentrations of various biofluids.

Serum and urine both generate high fluorescence, which increases with the increase of concentration. In contrast, saliva emits no fluorescence. Excitation wavelength: $358 \mathrm{~nm}$, and emission wavelength: $405 \mathrm{~nm}$.
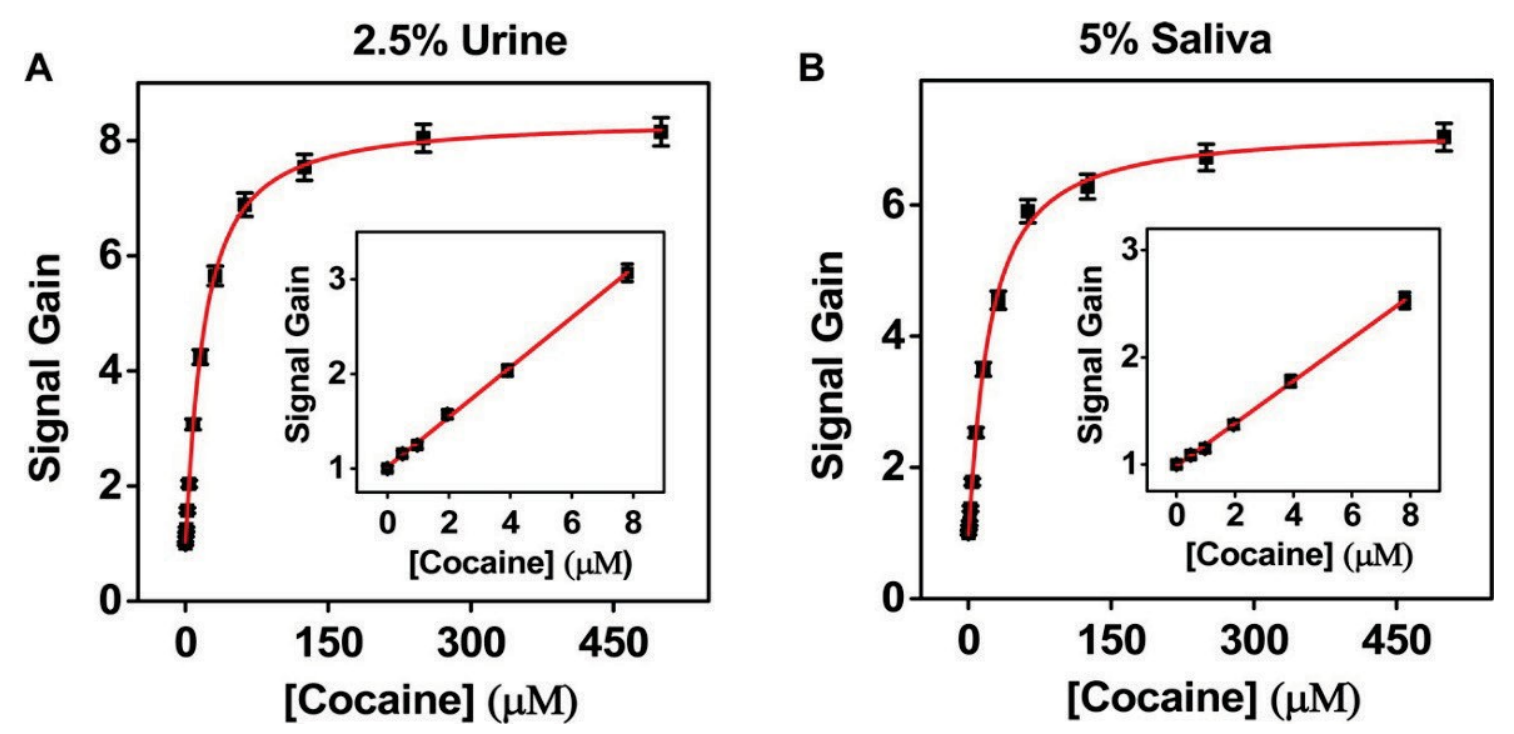

Figure 18. Calibration curves for ATMND-38-GC-displaced cocaine detection in biological samples.

We observed a strong concentration-dependent response to cocaine in (A) $2.5 \%$ urine and in (B) $5 \%$ saliva. Experimental conditions: [DNA] $=2 \mu \mathrm{M}$, [ATMND] $=250$ $\mathrm{nM}$. Error bars represent the standard deviation of three measurements conducted at each cocaine concentration. 
Serum samples generate weak background fluorescence (Figures 16 and 17). However, since serum contains $\sim 67 \mathrm{mg} / \mathrm{mL}$ protein, ${ }^{164}$ which might bind ATMND molecules within hydrophobic patches and thereby quench its fluorescence (Figure 19), we expected these samples to be significantly challenging to work with. Even though serum samples demonstrated considerable ATMND quenching at $405 \mathrm{~nm}$ (Figure 19), we were still able to detect cocaine in $2.5 \%$ serum with a detection limit of $900 \mathrm{nM}$ (Figure 20). Saliva is a cleaner matrix with no background fluorescence (Figure 17), containing only $\sim 1.6 \mathrm{mg} / \mathrm{mL}$ proteins, ${ }^{165}$ and we were thus able to use $5 \%$ saliva to achieve cocaine detection and establish a calibration curve with an LOD of $520 \mathrm{nM}$ (Figure 18B). Based on these findings, we calculated detection limits of $10.4,18.4$, and $36 \mu \mathrm{M}$ in undiluted saliva, urine, and serum, respectively. These experiments successfully demonstrate the feasibility of using our sensor to detect cocaine in body fluids.

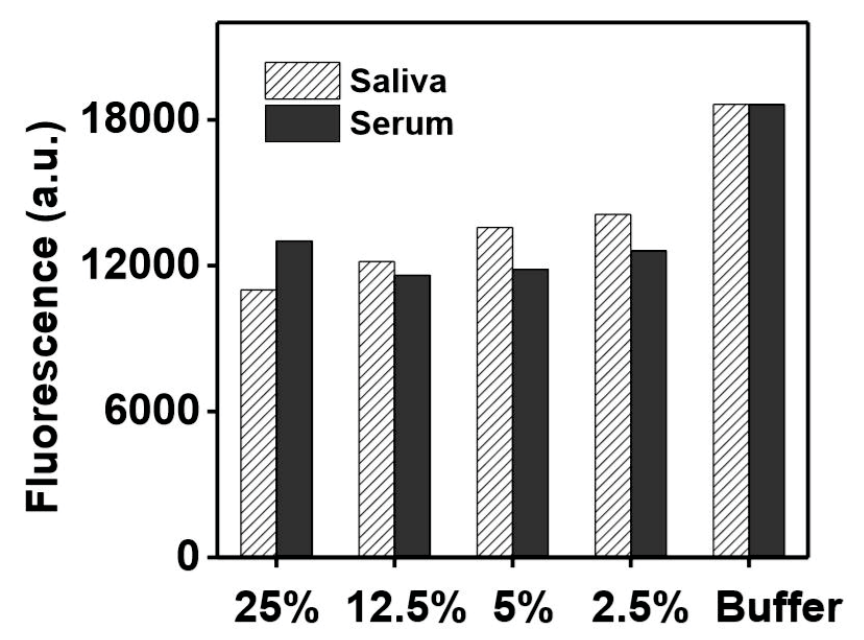

Figure 19. Fluorescence intensities of ATMND in different concentrations of saliva and serum.

The fluorescence of ATMND was quenched by higher concentrations of saliva and serum. Excitation wavelength: $358 \mathrm{~nm}$, and emission wavelength: $405 \mathrm{~nm}$. 

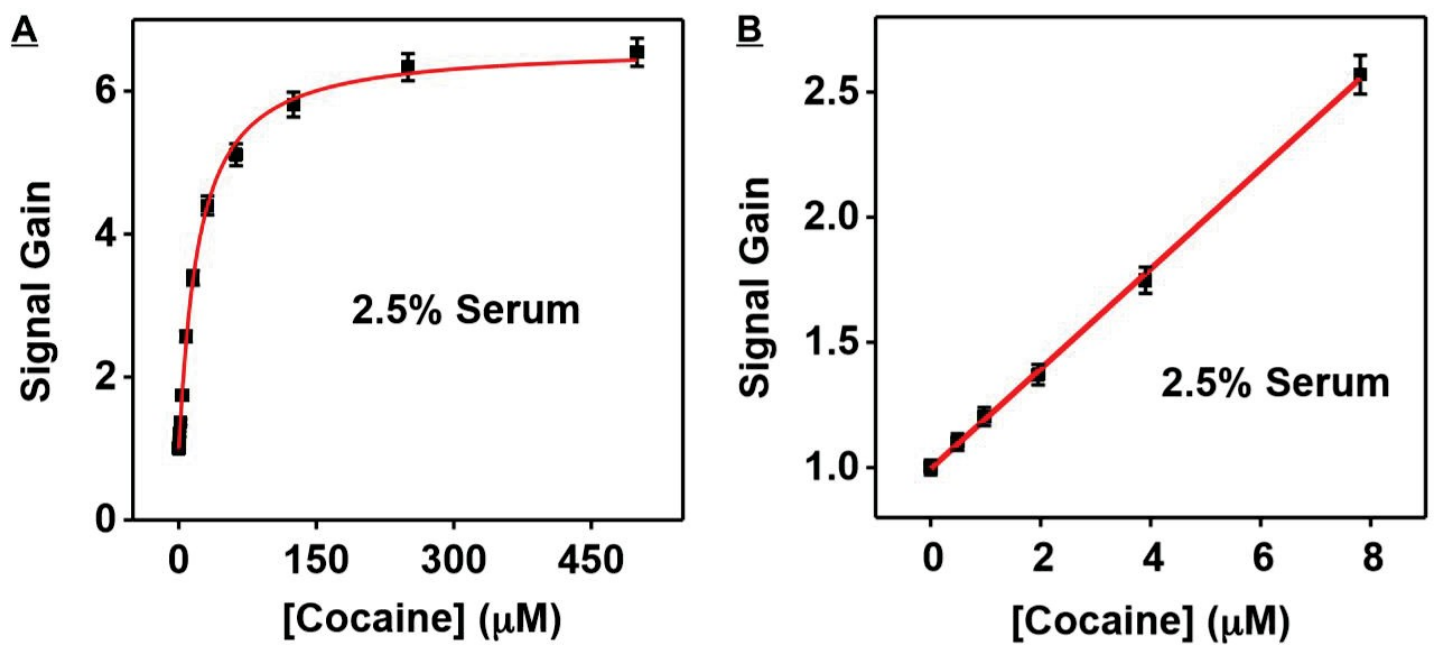

Figure 20. Calibration curves for cocaine detection with ATMND-38-GC in $2.5 \%$ serum.

(A) Concentration-dependent response of signal gain to cocaine concentration.

(B) Linear relationship between signal gain and cocaine concentration within the range from 0 to $8 \mu \mathrm{M}$. Experimental conditions: [38-GC] $=2 \mu \mathrm{M}$, [ATMND] $=250$ $\mathrm{nM}$, with excitation at $358 \mathrm{~nm}$ and emission at $405 \mathrm{~nm}$. Error bars represent the standard deviation of three measurements conducted at each cocaine concentration.

\subsubsection{Detection in alcoholic and soft drinks}

We further used the ATMND-cocaine test to measure cocaine in drinks such as beer and soda. Detection was performed in $10 \%$ diluted solutions with spiked cocaine (250 uM). Detection in several soft drinks proved difficult because of intense background fluorescence, however detection was still possible in drinks such as tea, Coca-Cola and soda (Figure 21). Detection of spike cocaine was also tested in alcoholic drinks. Several alcoholic drinks resulted in unreliable detection because of high background fluorescence even in the absence of cocaine. Detection was still possible in several diluted alcoholic drinks (Figure 22). The outlined method thus can successfully detect cocaine in spiked drinks, however care should be taken to examine the background fluorescence of the matrix. 

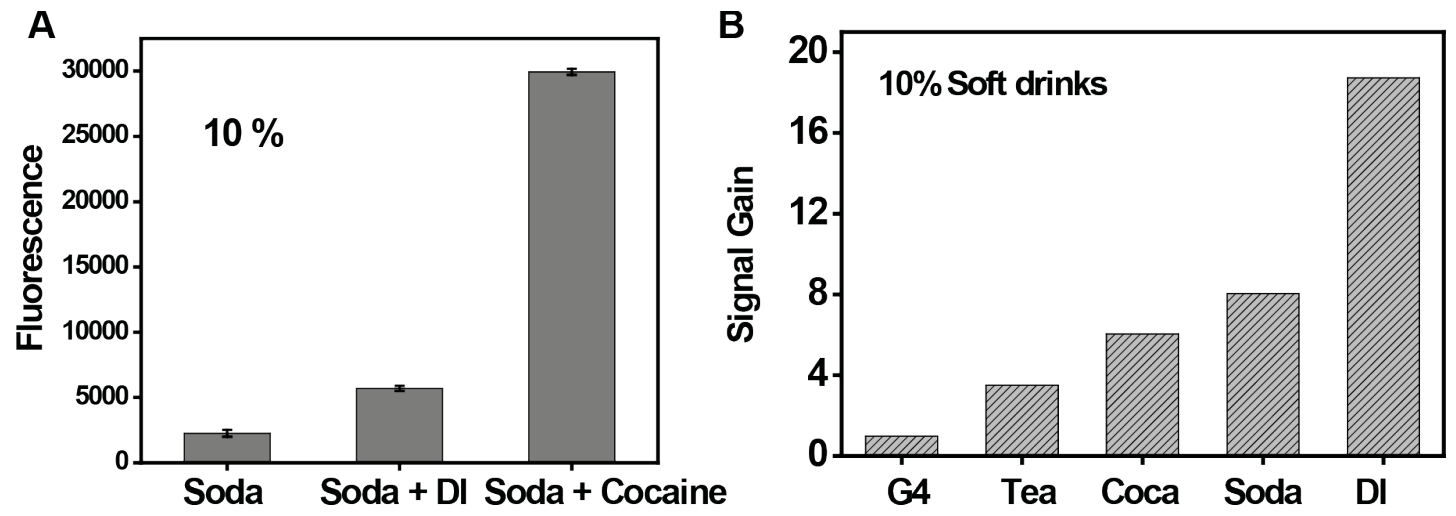

Figure 21. Detection of cocaine in spiked soft drinks.

(A) fluorescence intensity of ATMND in soda solution. Addition of cocaine results in a significant fluorescence increase because of the spiked cocaine in the solution.

(B) Different soft drinks were tested with only Gatorade resulting in unreliable detection of cocaine. [DNA] $=2 \mathrm{uM},[$ ATMND] $=250 \mathrm{nM},[\mathrm{COC}]=250 \mathrm{uM}, \mathrm{Ex}: 358$ nm, Em: 405 nm.

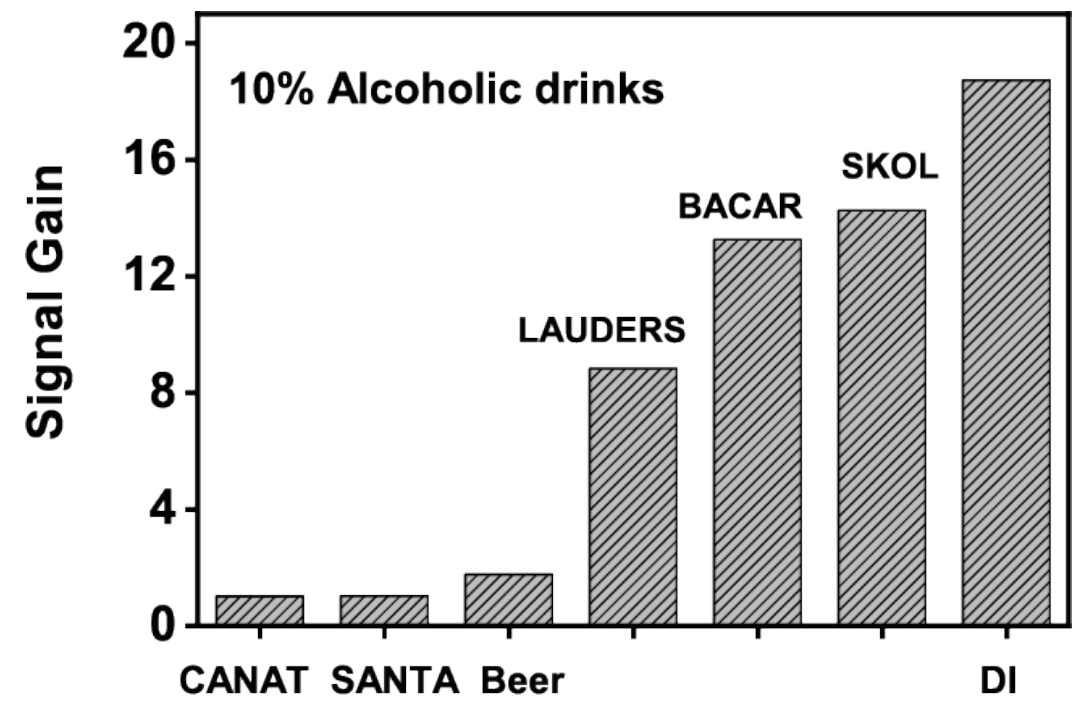

Figure 22. Detection of cocaine in alcoholic drinks.

Several drinks were diluted to $10 \%$ and spiked with cocaine. Detection was performed with the ATMND-method resulting in correct detection for several drinks. However, several drinks exhibit intense fluorescence that results in unreliable results. Care should be taken to examine the fluorescence signature in a case by case basis. $[\mathrm{DNA}]=2 \mathrm{uM},[\mathrm{ATMND}]=250 \mathrm{nM},[\mathrm{COC}]=250 \mathrm{uM}, \mathrm{Ex}$ : 358 nm, Em: 405 nm. 


\subsection{Conclusion}

We have demonstrated the rapid, sensitive, and specific detection of cocaine based on competitive aptamer binding between the cocaine target and the ATMND fluorescent signal reporter. Our 38-GC aptamer is derived from the previously reported MNS-4.1 aptamer and incorporates additional complementary base pairs at multiple sites that stabilize aptamer folding and thereby increase binding affinity to both ligands and reduce background fluorescence. ATMND fluorescence is significantly quenched upon binding to $38-\mathrm{GC}$. In the presence of cocaine, however, ATMND is displaced from the dye-aptamer complex, generating an intense fluorescence signal. We confirmed the competitive binding of the two ligands to the $38-G C$ and demonstrated that targeted mutagenesis clearly affects sensor performance.

Our assay is remarkably simple, fast, and specific. Detection is performed in a single tube containing the 38-GC-ATMND complex and the sample of interest. The assay is label-free, and detection only requires $20 \mathrm{~s}$ at room temperature to achieve a linear range of $0-8 \mu \mathrm{M}$ with an LOD of $200 \mathrm{nM}$ in buffer, about 50 -fold lower than assays based on target-induced conformational change. ${ }^{11,57,59,64,140-144}$ More importantly, our assay can achieve successful cocaine detection in body fluids. We observed that the signal gain increased with increasing cocaine concentrations, obtaining LODs of $10.4,18.4$, and $36 \mu \mathrm{M}$ in undiluted saliva, urine, and serum samples, respectively. After a dose of $100 \mathrm{mg}$, it is reported that cocaine concentration is around $2 \mathrm{mg} / \mathrm{L}$ in urine, about 3 times lower than the LOD of our sensor. ${ }^{166}$ Thus, a major limitation of the current assay is the interference by auto- 
fluorescence of the biological sample which limits the sensitivity. This could be possibly overcome by utilizing a dye outside the $358 / 405 \mathrm{~nm}$ window. We anticipate that the well-established SELEX technique should likewise make it possible to derive similar target-ligand displacement sensors that exhibit equally high specificity and affinity for other small molecules. This approach therefore potentially offers a general framework for performing rapid and specific highthroughput on-site drug testing. 


\section{CHAPTER 4}

\section{Engineering an adenosine-specific aptamer and electrochemical detection in undiluted serum}

A simple and specific method for the detection of adenosine is becoming increasingly valuable as knowledge of its modulatory function in physiology and pathophysiology are explicitly understood. Unfortunately, the previously reported Adenosine aptamer (ATP-27) cannot be utilized to measure adenosine concentration because of its inherent cross-reactivity to adenosine phosphate derivatives such as adenosine triphosphate (ATP) and adenosine diphosphate (ADP), which share a common core structure (ribose and adenine) and are copresent in biological samples. Through rational engineering of the aptamer's sequence, we designed and characterized a new highly specific adenosinebinding aptamer which differs from ATP-27 by a single nucleotide and exhibits reduced cross-reactivity for ATP, ADP and adenosine monophosphate (AMP). The reduced cross-reactivity can be attributed to steric hindrance for negatively charged phosphate derivatives in the binding pocket, resulting in decreasing affinity as the negative phosphate charge increases, yet neutral adenosine is not affected. The aptamer can be modified and incorporated into an electrochemical aptamer-based (E-AB) platform for sensitive and specific detection of adenosine in buffer and undiluted serum. Sensitivity in serum is at least 40 times higher compared to other aptamer-based sensors but with specificity for adenosine against ATP, ADP and AMP that has not been previously reported in aptamerbased serum measurements. 


\subsection{Introduction}

Adenosine is an endogenous regulatory molecule with signaling function in diverse systems including immune and inflammation response, neuro-modulation, coronary blood flow and cancer pathogenesis. ${ }^{73,84,167}$ Membrane equilibration channels transport adenosine to the outside of the cell, however the bulk of extracellular adenosine is produced by metabolism of nucleotides (ATP, ADP and AMP) by membrane-bound ecto-enzymes (i.e., CD39 and CD73). ${ }^{123}$ The enzymes work in concert to transform ATP to adenosine, first by transformation of ATP to AMP by CD39 followed by metabolism of AMP to adenosine by CD73.$^{85}$ Intracellularly, adenosine homeostasis is regulated by enzymes such as adenosine deaminase (ADA: transforms adenosine to Inosine) and adenosine kinase (ADK: transforms adenosine to AMP). ${ }^{168}$ Extracellular adenosine can regulate physiological functions by binding to and activating four distinct adenosine receptors $\left(A_{1}, A_{2 a}\right.$, $A_{2 b}$ and $A_{3}$ ) expressed in nearly all cell types. ${ }^{75}$ Physiological concentration of extracellular adenosine (30-300 nM) can effect regulatory functions with the highaffinity receptors $\left(A_{1}, A_{2 a}\right.$, and $\left.A_{3}\right)$ but not with the low affinity receptor $A_{2 b}{ }^{169}$ Activation of the $A_{2 b}$ receptor by adenosine requires concentrations $100-1,000$ times above physiological levels (>10 uM), which are only observed in pathological states such as hypoxia, tissue damage, cell-stress and cancer. ${ }^{74,76,170}$ Accordingly, adenosine measurements have been used to diagnose diseases, monitor therapeutic efficacy, understand the role of purinergic enzymes and adenosine receptors in pathophysiology, and to indirectly quantify adenosine regulatory enzymes and profile potential inhibitors of these enzymes. ${ }^{77,78,171-173}$ For example, 
Corcuilo et al. proved that mice lacking the adenosine receptor $A_{2 a}$ or the enzyme CD73 (transforms AMP to adenosine), spontaneously develop osteoarthritis with replacement of adenosine by injection preventing development of osteoarthritis in rats. ${ }^{174}$ Taniai et al. demonstrated higher concentrations of adenosine in the urine of patients suffering from metabolic disease or severe respiratory failure. ${ }^{171}$ Bimal et al. demonstrated elevated plasma adenosine levels during active visceral leishmaniasis $(\mathrm{VL})$ disease and return to basal adenosine levels after successful treatment. ${ }^{172}$ An important aspect of these studies was utilization of a reliable method for adenosine measurements. Currently available methods include microdialysis, HPLC, enzyme-linked spectroscopic assays, and chromatography-MS based methods. ${ }^{77,80,82,175}$ These methods require complex procedures, expensive instruments, lengthy sample processing, and have limited temporal resolution. Chen et al. argued that a current challenge in therapeutic targeting of adenosine receptors is the unavailability of simple and direct adenosine sensors. ${ }^{75}$

Various groups have attempted to develop simple sensing methods to measure adenosine concentrations with high specificity. Ahn et al. developed a chemical method that indirectly measured adenosine by employing sadenosylhomocysteine-hydrolase to transform s-adenosylhomocysteine to homocysteine and adenosine. Homocysteine results in fluorescence quenching of a silver nanocluster and exogenous adenosine is indirectly measured as it inhibits the enzymatic reaction of s-adenosylhomocysteine-hydrolase preventing production of the homocysteine which regulates the fluorescence output. The method showed great specificity against ATP and ADP in 5\% diluted complex 
samples, however the method was not tested in undiluted serum. ${ }^{176}$ Additionally, the method requires exogenous reagents and time-consuming enzymatic reactions which limit the temporal resolution. ${ }^{177}$ Biovision has a commercially available fluorescence kit for indirect detection of adenosine in serum and urine. Biovision's method relies on transformation of adenosine by adenosine deaminase to inosine and a downstream enzymatic transformation (not publicly available) of the resulting product for fluorescence detection. ${ }^{178}$ While highly sensitive, the method is not highly specific, with intense background interference from endogenous inosine and xanthine, molecules which are commonly present in biological samples. ${ }^{179}$ Other methods have also been developed based on enzyme recognition of adenosine and cascading catabolism of the target molecule to eventually produce hydrogen peroxide, which can be measured using electrochemistry or spectroscopy. ${ }^{81,180}$ Specifically for electrochemical sensors, enzyme immobilized electrodes require specialized and careful preparation and are inherently sensitive to interfering electroactive molecules in the sample solution because of the interrogating potential of $+500 \mathrm{mV}$ required for oxidation of hydrogen peroxide which also oxidizes molecules endogenously present in biological samples such as ascorbate. ${ }^{81,180}$ Specific detection of adenosine is also affected by inosine concentration (adenosine metabolite) which must be subtracted by a separate inosine reference sensor. While these methods are more convenient than conventional methods (e.g. HPLC), they do not measure adenosine directly, thus they suffer from poor specificity against matrix interferents. 
Aptamer-based sensors are increasingly gaining popularity because of their high and tunable specificity, adaptability to highly selective sensing mechanisms and high sensitivity. $9144,181,182$ Aptamers are oligonucleotide-based recognition molecules that can bind specific analytes such as small molecules and proteins. ${ }^{38,39}$ An analyte of interest, such as adenosine, is incubated with a large population of random and distinct oligonucleotide sequences of fixed length, termed a 'library'. Distinct sequences in the library that strongly bind the analyte are continuously amplified (i.e., enriched) and non-binding sequences are discarded; this artificial evolution process (termed SELEX) is repeated until a unique binding sequence is isolated. ${ }^{39}$ The specificity of the isolated sequence can be controlled by appropriate design of the incubation and separation steps; thus aptamers can be made to be highly specific for a given molecule or cross-reactive for a family of molecules, depending on the selection conditions. ${ }^{139,183}$ Interestingly, the previously isolated ATP-binding aptamer (herein termed ATP-27) is highly cross-reactive with ATP, ADP, AMP, and adenosine with little interaction with the mono/di/tri-phosphate portion of the adenosine molecule. ${ }^{47,184-187}$ The full molecular structures are presented in figure 1. Biological fluids contain mixtures of ATP, ADP, AMP and adenosine because of the sensitive homeostasis mechanism of cell energy consumption and signaling.${ }^{75}$ Indeed, ATP is present at higher concentrations intracellularly ( $\mathrm{mM}$ vs $\mathrm{nM}$ ) and extracellularly as compared to adenosine, thus utilization of the current ATP-27 aptamer for specific sensing of adenosine is not possible. ${ }^{73,76}$ Unfortunately, most aptamer-based sensors for adenosine have focused on improving the sensitivity or the specificity against 
matrix interferences, but have largely ignored the cross-reactivity problem that prohibits the use of the current aptamer for measurements in biological fluids. ${ }^{48,79,188}$ For example, time-resolved spectroscopy and flow cytometry aptamer-methods have achieved detection of adenosine in complex serum samples. However, these methods were not able to overcome the specificity problem of the ATP-27 aptamer and additionally sacrificed analytical performance with up to 10-20 times reduced binding affinity for adenosine, limiting their utility for clinical applications (sensitivity: 60-200 uM). ${ }^{46,189}$ Several groups have attempted to isolate more specific aptamers ATP, ADP and adenosine..$^{86,190,191}$ For example, an ATP specific RNA aptamer was isolated however this aptamer interacts strongly with the phosphate backbone resulting in unwanted crossreactivity with guanosine triphosphate (GTP). ${ }^{191}$ Stojanovic et al. reported a unique adenosine binding aptamer that can bind adenosine and cyclic-AMP but does not bind any other adenosine-phosphate derivatives ${ }^{86}$ Unfortunately, apart from the selection publication there is no additional information available about this aptamer, characterization of its structure or its application in complex sample measurements. The current limitation for a functional aptamer-based sensor for adenosine detection is thus the inability to design a sensing platform that can simultaneously overcome the issues of specificity against similar molecules (i.e., cross-reactivity), specificity against matrix interferents and reach an appropriate level of sensitivity (e.g., micromolar).

To simultaneously overcome these challenges, an adenosine-specific aptamer must be developed and coupled to a highly selective and highly sensitive signaling 
mechanism. Through rational sequence engineering of the original ATP-27 aptamer, I discovered a new oligonucleotide sequence that can specifically bind to adenosine and has reduced interactions with ATP, ADP and AMP. Additionally, the new adenosine-specific aptamer has a similar stem-loop structure as the original aptamer and it can be easily adapted to various signaling methods including electrochemistry. Electrochemical aptamer-based sensors (E-AB) can be utilized directly in complex samples (e.g., undiluted serum) with target-dependent response that is largely insensitive to interfering molecules in the matrix but whose cross-reactivity is characterized by the employed aptamer. ${ }^{93,192-194}$ The aptamer modulates the measured current by changing its conformation (i.e., secondary structure) upon target binding, which changes the distance and mobility of a covalently-attached redox molecule to the electrode surface..$^{92,97,195,196}$ Signaling only occurs through specific target-induced changes in the aptamer's secondary structure and is therefore mostly irresponsive to non-binding molecules and adsorption of contaminants to the electrode surface. Additionally, the scarcity of electroactive molecules in complex samples such as blood ensures interferencefree detection, as opposed to optical-based sensors which suffer from significant interference from non-target absorbing and fluorescent molecules. ${ }^{176,182}$ Our method exhibits high specificity against similar molecules (e.g., vs. ATP, ADP and AMP), high specificity against matrix interferents even in undiluted serum and high sensitivity, simultaneously overcoming the limitations of current aptamer-based sensors for adenosine. I discuss a probable explanation for the enhanced specificity of the new adenosine-specific aptamer, its significance for future sensor 
design and for the first time report an aptamer-based method for specific adenosine detection in undiluted serum, as evidence of its possible application in the medical and research fields.

\subsection{Experimental Section}

Reagents

Adenosine, adenosine triphosphate (ATP), adenosine diphosphate (ADP), adenosine monophosphate (AMP) and guanosine triphosphate (GTP) were purchased from Sigma and dissolved to $10-50 \mathrm{mM}$ concentration in deionized water (DI) and neutralized with sodium hydroxide. Approximate concentrations were obtained from absorption measurements at $259 \mathrm{~nm}$ and utilization of the appropriate extinction coefficient. Exonuclease I (Exo I) and Exonuclease III (Exo III) were purchased from New England Biolabs (NEB) and stored at $-20^{\circ} \mathrm{C}$ in $5 \mathrm{uL}$ aliquots of stock solution of $100 \mathrm{U} / \mu \mathrm{L}$. Oligonucleotide DNA aptamers were acquired from IDT DNA technologies with high-performance liquid chromatography (HPLC) purification in most cases except for isothermal titration calorimetry and circular dichroism experiments, which used standard desalted DNA. Methylene-blue (MB) modified DNA was purchased from Biosearch with HPLC purification. All DNA was dissolved using nuclease free water; the approximate concentration was measured at $260 \mathrm{~nm}$ with the corresponding extinction coefficient of each DNA sequence and stored at $-30{ }^{\circ} \mathrm{C}$ until used. The DNA sequences utilized are listed in the following table: 
Table 3. Aptamer sequences, modifications and substitutions.

\begin{tabular}{l|l}
\hline \multicolumn{1}{c}{ Name } & \multicolumn{2}{c}{ Sequence (5' - 3') } \\
\hline ATP-33 & CGC ACC TGG GGG AGT ATT GCG GAG GAA GGT GCG \\
ATP-33m & CGC ACC TGG GGA AGT ATT GCG GAG GAA GGT GCG \\
ATP-30 & CGC ACC TGG GGG AGT ATT GCG GAG GAA GGT \\
Ade-33 & CGC ACC TGG GGG AGT ATT GCG GTG GAA GGT GCG \\
Ade-33m & CGC ACC TGG GGA AGT ATT GCG GTG GAA GGT GCG \\
Ade-30 & CGC ACC TGG GGG AGT ATT GCG GTG GAA GGT \\
Ade-30m & CGC ACC TGG GGA AGT ATT GCG GTG GAA GGT \\
Ade-29 & CGC ACC TGG GGG AGT ATT GCG GTG GAA GG \\
Ade-29-MB & (HS-C6)- CGC TCC TGG GGG AGT ATT GCG GTG GAA GG TTT -(C6-MB) \\
\hline
\end{tabular}

\subsubsection{Polyacrylamide gel electrophoresis (PAGE)}

Denaturing polyacrylamide gel electrophoresis was utilized to determine the length of the DNA products of enzyme reactions. In all cases, $15 \%$ gels were used to provide optimal resolution and band separation. Gel preparation consisted of mixing $9.4 \mathrm{~mL}$ of $40 \%$ acrylamide solution, $5 \mathrm{~mL}$ of $5 X$ Tris-Borate-EDTA (TBE), $2.6 \mathrm{~mL}$ of deionized water (DI), $100 \mu \mathrm{L}$ of freshly prepared $10 \%$ ammonium persulfate (APS) $(w / v)$, and $10 \mu \mathrm{L}$ of tetramethylethylenediamine (TEMED). Gels were left to polymerize for 2 hours before pre-running at $450 \mathrm{~V}$ for 30 minutes with $0.5 X$ TBE running buffer. Samples were loaded at $3 \mu \mathrm{L}$ per well ( 20 wells per gel). The running conditions were 30 mins. at $100 \mathrm{~V}$ and 2 hours at $400 \mathrm{~V}$. The sample bands were compared to a DNA control without enzyme treatment to approximate the concentration of the resulting product. A marker lane was used to estimate the product nucleotide count.

\subsubsection{Enzyme reactions}

A DNA stock solution of $50 \mu \mathrm{M}$ was heated to $95{ }^{\circ} \mathrm{C}$ and then cooled on ice for 5 minutes. The DNA was mixed in buffer solution (10 mM Bis-tris-propane, $1 \mathrm{mM}$ 
$\mathrm{MgCl}_{2}, 1 \times$ Bovine Serum Albumin (BSA) at $\mathrm{pH} 8.5$ ) and the appropriate target was added (e.g., adenosine). The target and aptamer were incubated at $23^{\circ} \mathrm{C}$ for 30 minutes in a heating plate. Exonuclease I and exonuclease III were diluted at varying concentrations with $1 \mathrm{X}$ BSA buffer (10 mM BTP, 1x BSA at pH 8.5) unless otherwise indicated and were then added to the aptamer-target mixture. The reactions were performed at $23^{\circ} \mathrm{C}$ in $200 \mu \mathrm{L}$ PCR tubes for the time indicated. The reaction was stopped by mixing $5 \mu \mathrm{L}$ of the reaction mixture with $10 \mu \mathrm{L}$ of stop mix loading buffer (formamide and EDTA).

\subsubsection{Isothermal Titration Calorimetry (ITC)}

A Malvern ITC200 was used for the following experiments. The cell was loaded with the appropriate DNA sequence and the syringe was loaded with the small molecule ligand (e.g., adenosine). The concentration of DNA in the cell was varied between $15-30 \mu \mathrm{M}$ to achieve sufficient heat in each injection as well as saturation and quality fitting curves. The syringe concentration was restricted to between 450 - $600 \mu \mathrm{M}$ of each small molecule attributable to significant heat of dilution being observed at higher concentrations. The heat of dilution from a ligand to buffer run was subtracted from sample runs. A general experiment consisted of a $0.4 \mu \mathrm{L}$ purge injection, which was removed prior to data analysis, and 18 additional $2 \mu \mathrm{L}$ injections. In cases where saturation was not achieved in a single run, the syringe was reloaded with ligand and a second run was performed. The data were then aggregated onto a single file for fitting. It is noted that the DNA used for these experiments was only desalted with no other purification. Accordingly, an estimated concentration was utilized for the data fitting, with a probablefull-length 
oligo of $80 \%$ in the overall oligo mixture according to the manufacturer, IDT DNA. To fit the data, we used a sequential binding model based on previous literature which indicate a small factor of cooperativity observed in the original ATP aptamer which has been characterized to have two distinct, binding sites for ATP. ${ }^{197,198}$

\subsubsection{Circular Dichroism (CD)}

A Jasco J-815 CD was used for these experiments. The DNA concentration was

$2.5 \mu \mathrm{M}$ and the target molecule concentration was $25 \mu \mathrm{M}$. The target molecule concentration was restricted because of significant absorption of adenosine containing compounds at $259 \mathrm{~nm}$. The aptamer solution and aptamer-target solutions were prepared separately and incubated at $23{ }^{\circ} \mathrm{C}$ for 30 minutes to maintain the same conditions as those used for enzyme reaction experiments. We used standard sensitivity of the instrument and scanned the spectra form $300 \mathrm{~nm}$ to $215 \mathrm{~nm}$ with a data pitch of $0.1 \mathrm{~nm}$ at $50 \mathrm{~nm} / \mathrm{min}$. The given results are the average of 5 scans. We monitored the scans with 3 channels: CD, HT and Absorbance. The background from the absorption of buffer and small molecule target in buffer were subtracted from aptamer-target mixtures.

\subsubsection{Aptamer immobilization to gold electrodes}

The gold electrodes were first polished in alumina slurry for 5 minutes and subsequently sonicated in deionized water and absolute ethanol for 5 minutes each. The electrodes were then electrochemically cleaned following the protocol by Xiao et al. ${ }^{94}$ First, an electrochemical reduction/oxidation cycle was performed in $0.5 \mathrm{M}$ sodium hydroxide from $-0.35 \mathrm{~V}$ to $-1.35 \mathrm{~V}$ and rescanned 1,000 times. A 
subsequent round of oxidation/reduction steps were performed in $0.5 \mathrm{M}$ sulfuric acid $\left(\mathrm{H}_{2} \mathrm{SO}_{4}\right)$ and a final round was performed in $0.01 \mathrm{M}$ potassium chloride $\left(\mathrm{KCl} / 0.1 \mathrm{M} \mathrm{H}_{2} \mathrm{SO}_{4}\right)$. The working area of the electrode was determined in $0.05 \mathrm{M}$ $\mathrm{H}_{2} \mathrm{SO}_{4}$ by calculation of the reduction peak of gold oxide. ${ }^{199}$ The electrodes generally displayed a roughness $\sim 1.1$. Thiol-modified DNA was incubated with 10 $\mathrm{mM}$ tris(2-carboxyethyl)phosphine (TCEP) to reduce the disulfide bond. The reactive thiol DNA was then diluted to $645 \mathrm{nM}$ in 1x phosphate buffered saline (PBS) buffer. Aliquots of $200 \mu \mathrm{L}$ of the DNA mixture were incubated with the cleaned electrodes for a period of 4.5 hours in the dark at room temperature. The modified electrodes were then backfilled with $3 \mathrm{mM}$ 6-mercapto-1-hexanol (MCH), $1 \mathrm{mM} \mathrm{MCH}$ and $1 \mathrm{mM}$ dithiothreitol (DTT) or $1.5 \mathrm{mM}$ DTT for 3 hours to obtain stable monolayers of modified aptamers.

\subsubsection{Electrochemical measurements}

A CHI 760 bi-potentiostat was used for all measurements. $\mathrm{A} \mathrm{Ag} / \mathrm{AgCl}$ electrode was used as the reference electrode and a platinum wire was used as the counter electrode in a three-electrode set up. Square wave voltammetry (SWV) was employed to monitor the current as the potential was scanned from $-0.1 \mathrm{~V}$ to $-0.5 \mathrm{~V}$ in $10 \mathrm{mM}$ Bis-tris-propane, $50 \mathrm{mM} \mathrm{NaCl}$ buffer at $\mathrm{pH}$ 8.5. The frequency was set between $5-300$ hertz for optimization and $100 \mathrm{~Hz}$. was ultimately used as the optimal value. The amplitude was optimized to $25 \mathrm{mV}$ and the scan rate was $1 \mathrm{mV}$. The signal gain was calculated as the difference in peak current in the presence and absence of target divided by the target-free peak current. 
Calibration curves were collected by titrating increasing concentrations of target and final graphs were obtained from the average signal from at least 4 different electrodes in separate solutions. DNA surface coverage was measured using a previously reported protocol by Steel et al. ${ }^{200}$ Briefly, the modified electrodes were incubated in tris buffer ( $\mathrm{pH}$ 7.4) for 30 minutes prior to measurements. A small cell was filled with the same buffer and degassed using argon for 10 minutes prior to measurements. The electrodes were then immersed in the degassed buffer solution while keeping the purging gas on. Chrono-coulometry was employed to measure the absorption of ruthenium (III) hexamine and correspondingly calculate the concentration of DNA molecules adsorbed onto the gold electrode surface as described elsewhere. ${ }^{200}$

\subsection{Results and discussion}

\subsubsection{Cross-reactivity of the original ATP-binding aptamer}

Many publications have employed the 27-nt ATP-binding aptamer (herein termed ATP-27) to develop different, highly sensitive sensors for ATP, ADP and adenosine that can work in various samples matrices. ${ }^{184,201-203}$ However, in these publications, the inherent cross-reactivity of the ATP-27 aptamer for ATP, ADP, AMP and adenosine has been largely ignored or was inconsequential to the presented topic. I utilized the enzyme exonuclease III to probe the binding affinity of the various adenosine targets to the original cross-reactive ATP-27 aptamer. Exonuclease III has been recently reported to be inhibited by formation of aptamertarget complexes, generally 4-nt away from the presumed binding site. ${ }^{110}$ Thus, aptamer-target binding could be observed by gel electrophoresis of the enzymatic 
reaction products. A binding aptamer would produce bright bands corresponding to enzymatic inhibition and non-binding sequences would display no product bands as the enzyme would completely degrade the aptamer structure (fig. 23a). The exonuclease III system requires aptamers with long stabilizing stems, thus the original ATP-binding aptamer (ATP-27) was extended by three additional basepairs and termed ATP-33. Exonuclease III was heavily inhibited in the presence of binding targets, including ATP, ADP, AMP, and adenosine with retained products 30-nt long and with concentrations of 833, 810, 276 and $548 \mathrm{nM}$, respectively (fig. 23c). The retained product concentrations were calculated by comparison against the control band, which maintained a constant and known concentration. In the absence of target, exonuclease III displayed full activity and completely degraded the aptamer structure. Similar binding cross-reactivity of the ATP-27 aptamer has been reported with a variety of detection methods. ${ }^{185-187,189}$ A non-binding mutant sequence (ATP-33m) was utilized as a control with a single point mutation that inhibits binding to confirm that inhibition was indeed an effect of specific target binding and not due to enzymatic inhibition by the target itself. ${ }^{47}$ The ATP-33m non-binding sequence did not result in inhibition regardless of the presence or absence of target, which indicated that the target itself does not inhibit the enzyme (Fig. 23b). 


Ade (Neutral)

a

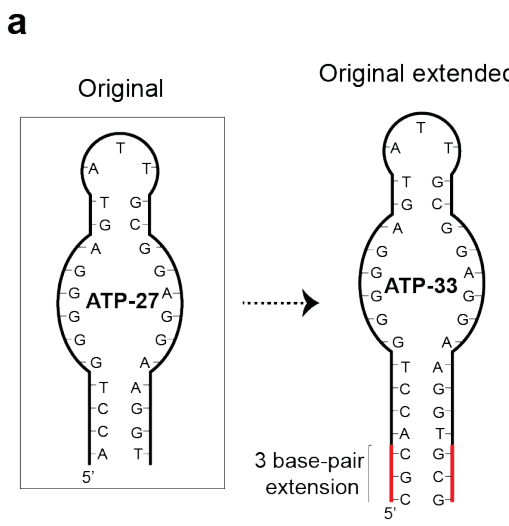

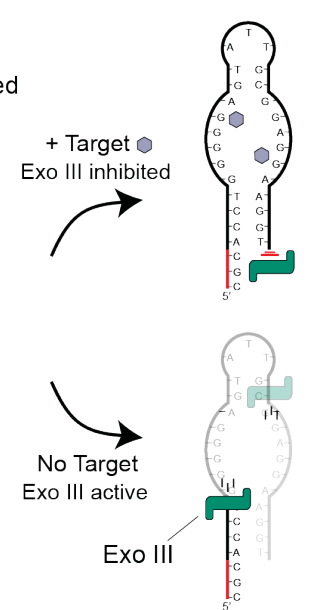

C

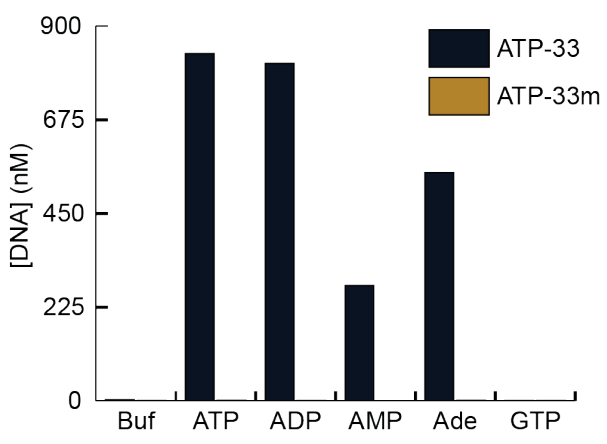

b ATP-33: Binding competent

ATP-33m: Non-binding mutant
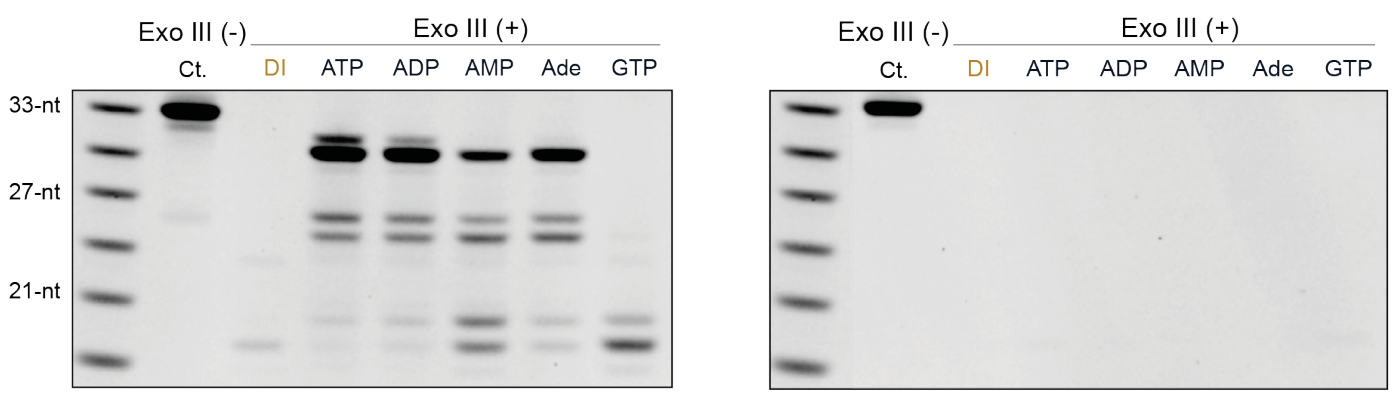

Figure 23. Specific target binding inhibited exonuclease III digestion of the aptamer 4-nt away from the presumed binding site.

(top) structure of adenosine, ATP, ADP, and AMP at pH 8.5. (a) The original ATP27 aptamer was extended by addition of 3 base-pairs to the stabilizing stem and the extended aptamer was termed ATP-33. Specific target binding inhibits exonuclease III digestion, while in the absence of target the enzyme can digest the aptamer. (b) Exonuclease III digestion products with the ATP-33 binding aptamer have an estimated length of 30-nt. Only the targets which bind can inhibit the enzyme. A mutant non-binding aptamer (ATP-33m) was used to confirm the binding-induced inhibition of exonuclease III. None of the targets inhibited exonuclease III. (c) The band intensity was converted to a concentration value by comparing with the control band of constant and known concentration. The binding-induced inhibition resulted in DNA inhibition with products of 833, 810, 276 and $548 \mathrm{nM}$ for ATP, ADP, AMP and adenosine, respectively. 


\subsubsection{Engineering of an adenosine-specific aptamer}

My goal was to improve the adenosine-specificity of the ATP-27 aptamer through rational sequence engineering, specifically via substitution of various nucleotides in the target-binding domain of the aptamer. Li et al. previously noted that the ATP27 cross-reactive aptamer exhibited strong binding affinity to ATP and ADP, but reduced binding affinity for AMP. The decreased binding affinity to AMP was explained by the higher negative charge on the a-phosphate of AMP (-2) as opposed to (-1) for ATP and ADP. The authors argued that the $\alpha$-phosphate might have close contact and interaction with the aptamer structure, thus the increased negative charge of the AMP $\alpha$-phosphate inhibited binding.$^{83}$ We hypothesized that rational mutation of the nucleotides near the binding site would modulate the specificity of the aptamer for the various adenosine phosphate derivatives because of their varying size and charge (molecular structures and charge displayed in figure 23). Multiple nucleotides in the sequence were mutated, added or removed and screened for target affinity via the exonuclease III inhibition system (fig. 24a). Through rational mutation of the original ATP-27 aptamer sequence, we discovered that a single nucleotide modification of an adenine at position 23 (counting from the 5 ' end) to a thymine inhibits binding of negatively charged adenosine derivatives (e.g., ATP, ADP, and AMP) but maintains affinity for neutral adenosine (Fig. 24b). Binding of adenosine to the new adenosine-specific aptamer (termed Ade-33) resulted in retention of $650 \mathrm{nM}$ of product while all other phosphate derivatives were almost fully digested (fig. 24c). A guided single-point mutation was introduced to the Ade-33 aptamer to obtain a non-binding control 
sequence termed Ade-33m. ${ }^{47}$ The non-binding Ade-33m was utilized to confirm that enzymatic inhibition was a result of specific target-binding. Complete digestion occurred in the presence of all targets, further confirming the specificity of the new aptamer for adenosine (Fig. 24b and c).

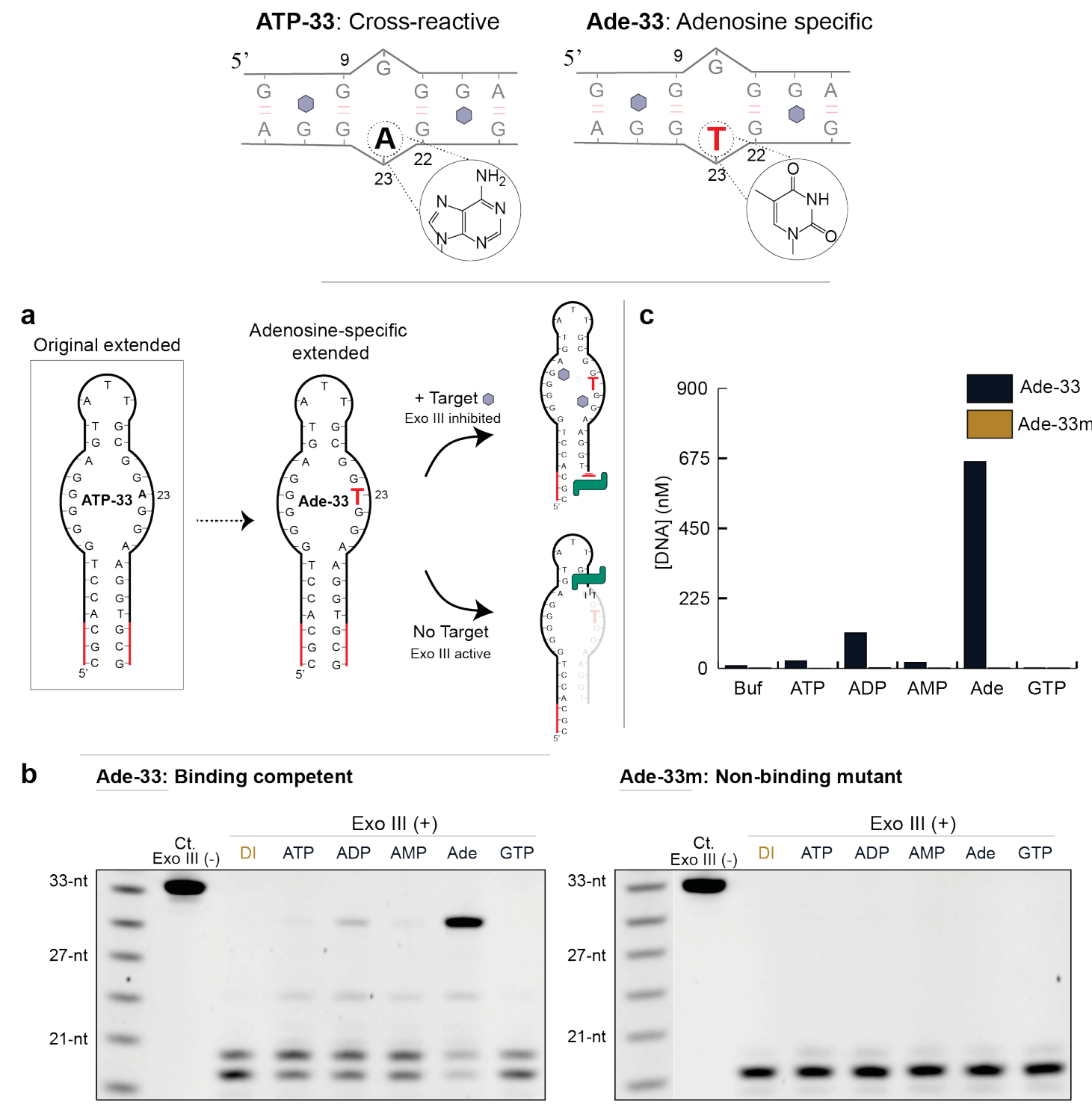

Figure 24. Exonuclease III screening test of engineered sequences and identification of an adenosine-specific aptamer termed Ade-33.

(a) Structures of the original cross-reactive ATP-33 aptamer and the new adenosine specific Ade-33 aptamer; A single nucleotide mutation at position 23 
(counting from the 5' end) of A to T can modulate the specificity of the aptamer for specific binding of adenosine. (b) Gel electrophoresis results of exonuclease III reaction with a binding competent Ade-33 aptamer and a mutated non-binding adenosine-specific aptamer (Ade-33m). (c) Concentration measurements of retained product after enzymatic reaction. Binding of adenosine retains over 650 $\mathrm{nM}$ of DNA product, while all other targets are almost completely digested. The mutated aptamer sequence is digested with all targets.

\subsubsection{Addition of Exonuclease I to remove background fragments}

Binding of the new adenosine specific aptamer can inhibit exonuclease III digestion but several aptamer fragments are still observed even in the absence of target or in experiments with a non-binding mutant. These short bands are single-stranded fragments without base-pairing complementary and are poor substrates for exonuclease III. I utilized the enzyme exonuclease I, which is highly specific for digestion of single-stranded DNA from the 3' end of the DNA sequence, to remove the background shorter fragments. A fixed concentration of exonuclease III was utilized to initially digest the parent aptamer along with increasing concentrations of exonuclease I to determine the concentration required to digest the remaining non-binding fragments. Addition of 3 units of exonuclease I was sufficient to remove the single-stranded DNA byproducts yet retain most of the aptamer-target complex product with nearly $450 \mathrm{nM}$ retained (fig. 25b). Interestingly, increasing concentrations of exonuclease I led to digestion of the 30-nt long aptamer-target complex, suggesting that the initial exonuclease III inhibition product is in a sensitive equilibrium between single- and double-stranded DNA conformations (fig. 25c). Next, the exonuclease I concentration was held constant and the exonuclease III concentration was varied to determine the minimum amount of 
exonuclease III required to obtain the binding inhibition product. As the exonuclease III concentration is reduced, a different binding product is observed (fig. 26). From these results, it appears that exonuclease III can recognize two different aptamer fragments that can undergo structure-switching upon target binding, with lengths of 30- and 29-nt (thus termed Ade-30 and Ade-29). Exonuclease I is inhibited only in the presence of target because of formation of double-stranded DNA upon target binding (fig. 26).

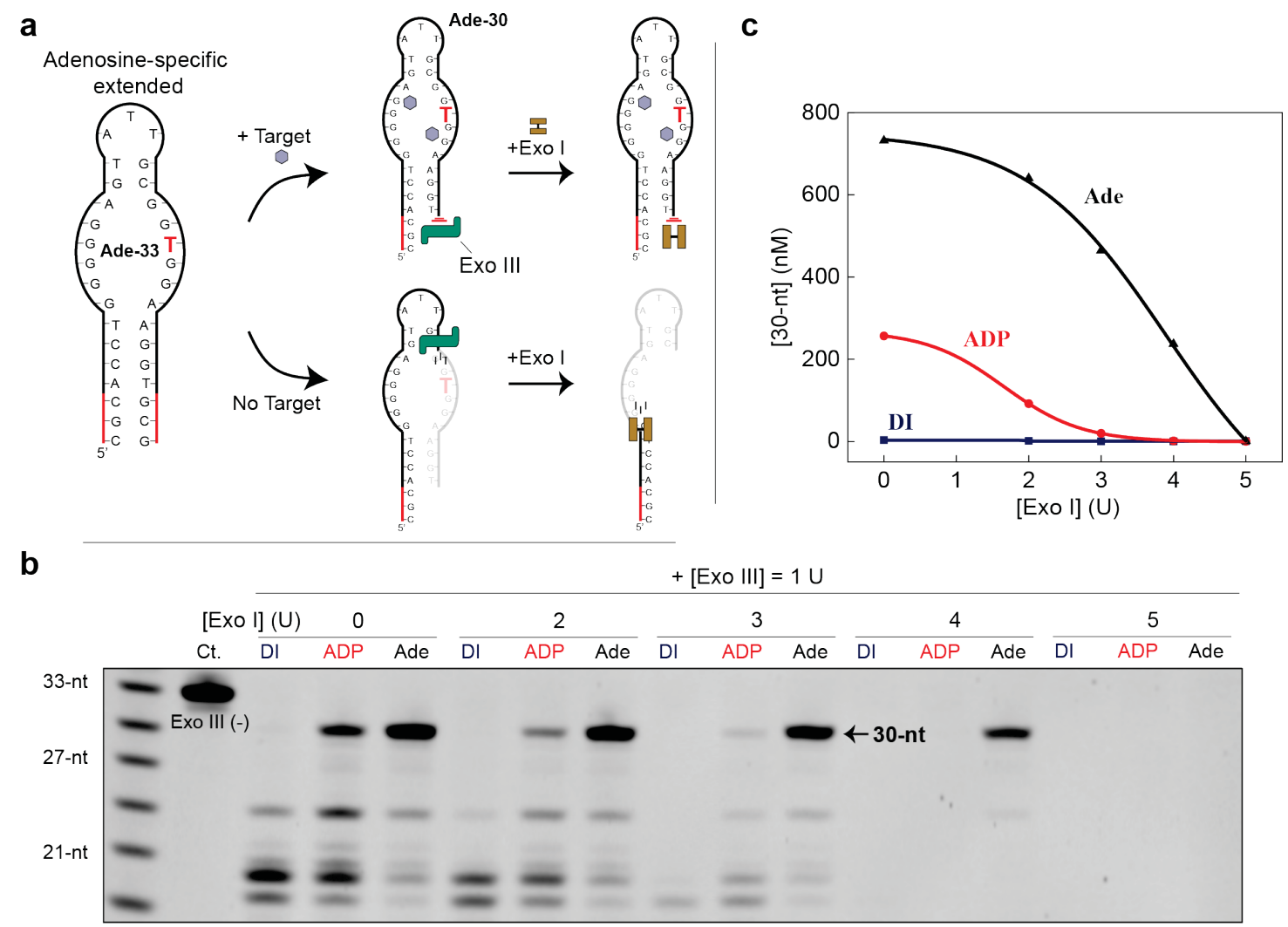

Figure 25. Addition of exonuclease I to remove single-stranded DNA fragments leftover from exonuclease III reaction.

(a) Exonuclease III can digest the structure in the absence of binding target up to a point where the structure becomes highly flexible single-stranded DNA, which is a poor substrate for exonuclease III. Exonuclease I is highly specific for such single-stranded DNA structures, allowing for digestion of the leftover fragments. 
(b) Gel electrophoresis results of increasing concentrations of exonuclease I at a fixed constant exonuclease III concentration. Exonuclease I can digest the leftover single-stranded DNA fragments, with $3 \mathrm{U}$ being sufficient to easily identify the aptamer-target complex. (c) Graphical representation of the band intensity as converted into concentration by comparison with a control band of known and constant concentration.

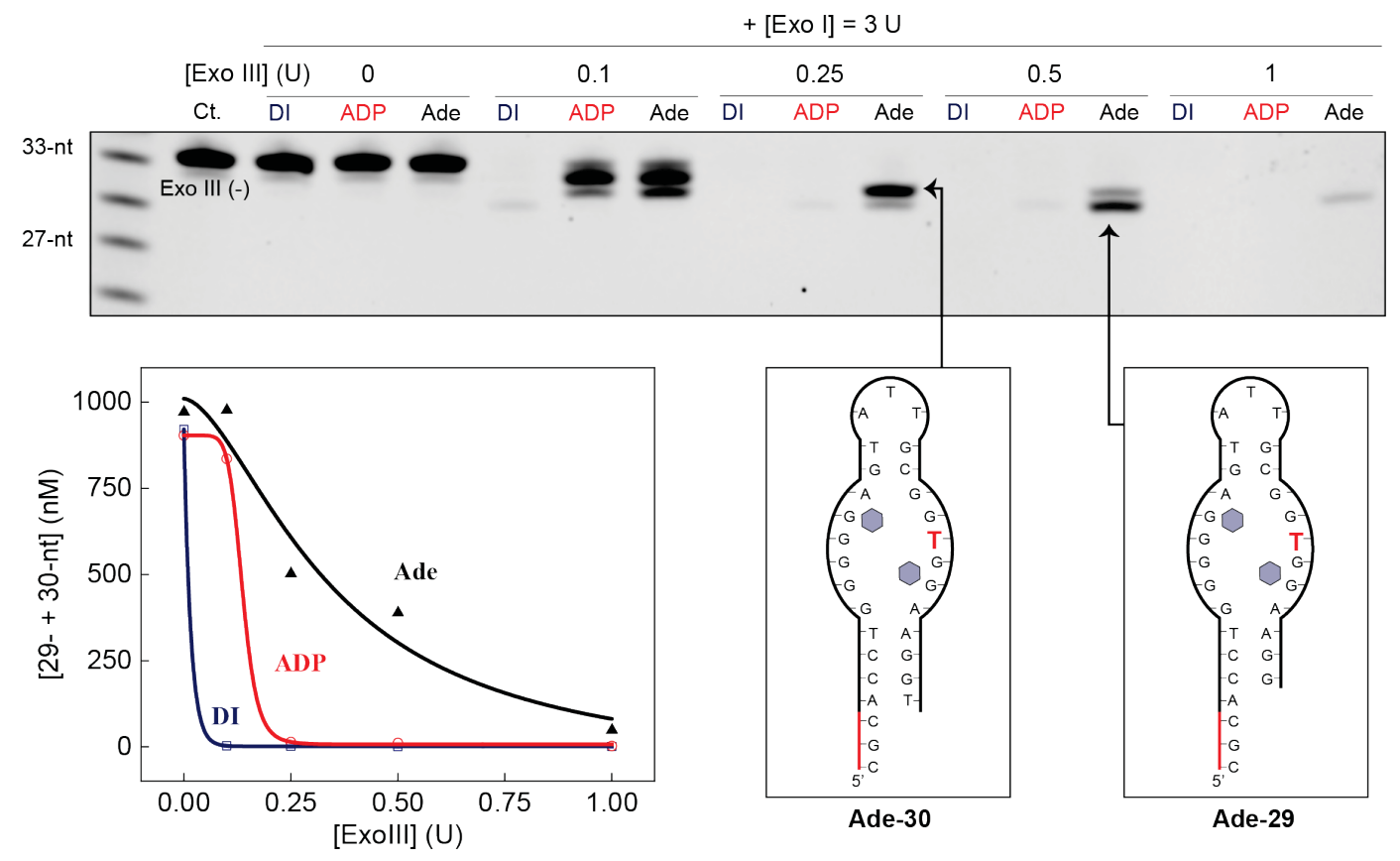

Figure 26. Exonuclease III can identify multiple aptamer fragments with apparent structure-switching functionality.

In the absence of exonuclease III, exonuclease I does not digest the extended Ade-33 structure as it is double-stranded structure. Upon digestion of exonuclease III, two different binding fragments are produced which appear to undergo structure-switching upon target-binding as they are not digested by exonuclease I, unlike samples without target-binding which are readily digested by exonuclease I.

\subsubsection{Implications of magnesium concentration on Ade-33 specificity}

ATP, ADP and AMP molecules binding to the adenosine-specific aptamer sequence appear more susceptible to steric interactions from the substituted thymine at position 23 . We performed the enzyme reactions with the Ade-33 
sequence at various magnesium concentrations to test whether magnesium affects binding between the thymine residue at position 23 and the phosphate containing adenosine molecules. At high magnesium concentrations (> $5 \mathrm{mM})$, the retention of Ade-33 products for adenosine and ADP was $<250 \mathrm{nM}$ while at low magnesium concentrations $(<5 \mathrm{mM})$ adenosine binding dominated and binding for ADP was severely reduced with retained products of $500 \mathrm{nM}$ for adenosine and none for ADP (fig. 27). We conducted the same test for the original cross-reactive ATP-33 aptamer and observed enzyme inhibition with ADP even at magnesium concentrations as low as $0.25 \mathrm{mM}$ (Fig. 27). Braun et al. previously demonstrated a similar effect of ATP binding to the cross-reactive ATP-27 aptamer at low magnesium concentrations. ${ }^{204}$ These results suggest that substitution of thymine at position 23 results in steric hindrance with the negatively charged phosphate groups of ATP, ADP and AMP. Thus, the Ade-33 aptamer was highly specific for adenosine at magnesium concentrations below $5 \mathrm{mM}$. At a concentration of $1 \mathrm{mM}$ magnesium, the ADP retained product is $600 \mathrm{nM}$ when bound to ATP-33 but no product is retained when incubated with the Ade-33 aptamer (fig. 27). Magnesium concentration above $5 \mathrm{mM}$ appears to be sufficient to attenuate the steric hindrance and stabilize binding of the negatively charged phosphate derivatives with the Ade-33 aptamer. ${ }^{205,206}$ A corresponding effect was previously observed by Szostak et al. who noted loss of ATP binding to an RNA aptamer in the absence of magnesium and an increase in specificity for ATP in the presence of increasing magnesium concentration. ${ }^{191}$ The authors proposed a role for magnesium where it functions as a bridging ion between their RNA aptamer structure and the ATP $\alpha$ - 
and $\beta$-phosphate groups. ${ }^{191}$ Similarly, a chemical sensor for ADP exhibits a metalion bridging mechanism which specifically binds ADP as compared to ATP and adenosine, by specifically targeting the phosphate groups in ADP. ${ }^{207}$ Stojanovic et al. previously reported a unique adenosine specific aptamer which is engineered to instill electrostatic hindrance between adenosine di/tri-phosphate and the DNA phosphate backbone, albeit with a different binding motif which only binds one molecule per aptamer ${ }^{86}$ I propose that substitution of adenine with thymine at position 23 in the Ade-33 aptamer increases electrostatic hindrance which results in reduced binding to the phosphate containing adenosine derivatives ATP, ADP and AMP (fig. 32). It is also possible that substituting thymine for adenine reduces stacking interactions with neighboring bases, destabilizing the binding pocket for the larger ATP and ADP molecules. ${ }^{208}$ Binding of the Ade-33 aptamer with negatively charged ADP was independent of sodium concentration, thus it is presumed that the effect of magnesium is primarily as a steric shielding/bridging molecule and not a result of differences in the DNA backbone stability (Fig. 28). 


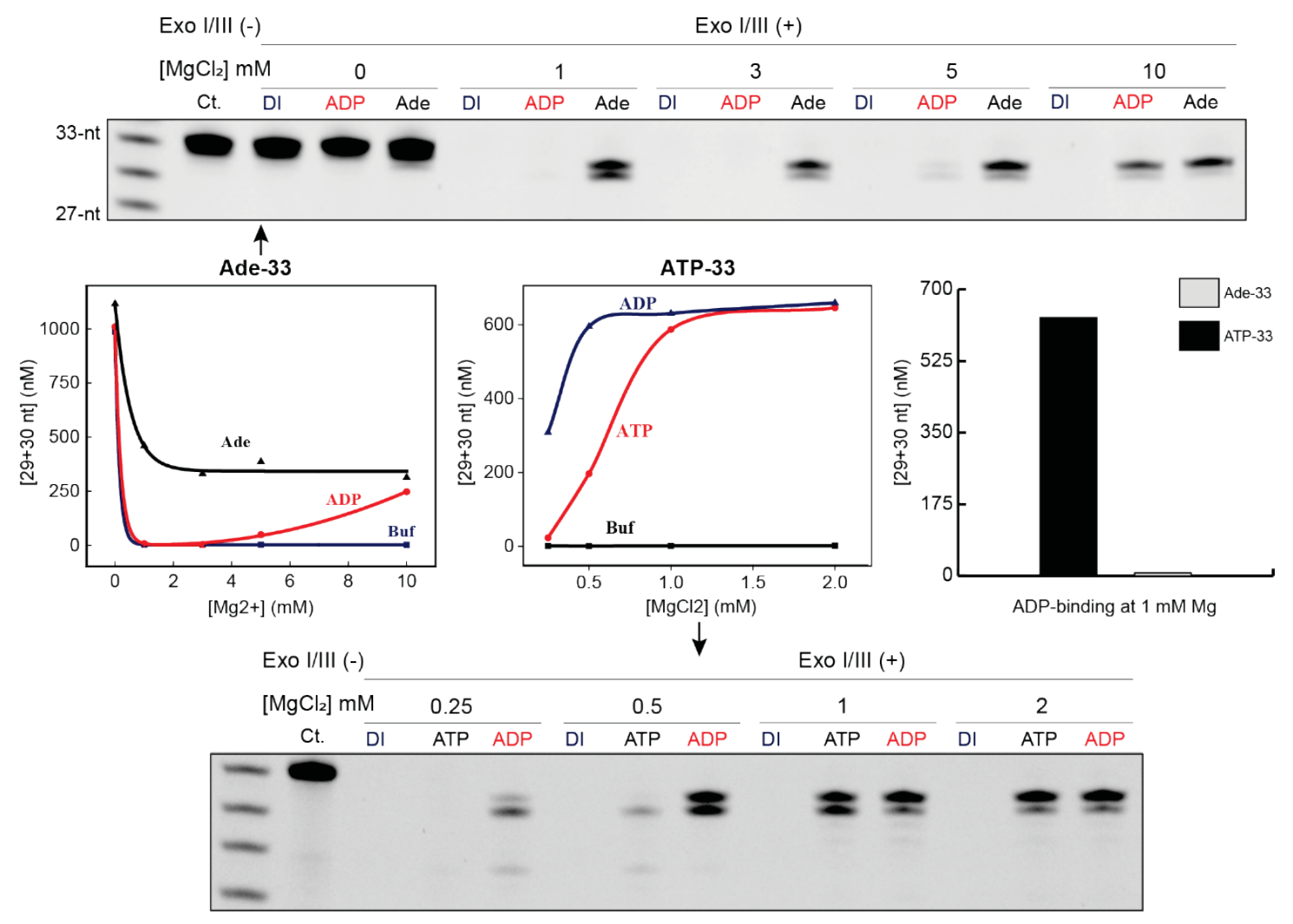

Figure 27. Magnesium effect in the binding specificity of Ade-33 and ATP-33 aptamers. (Top) The Ade-33 sequence was highly specific for Adenosine at magnesium concentrations below $5 \mathrm{mM}$ however at magnesium concentrations above $5 \mathrm{mM}$, presumed shielding by magnesium allows binding of ADP with the concentration of the retention product comparable to adenosine. (Bottom) The ATP-33 aptamer did not exhibit steric hindrance as magnesium concentrations as low as $0.25 \mathrm{mM}$ allowed for binding of ADP. The higher negative charge of ATP was susceptible to steric interactions but could be shielded at even $1 \mathrm{mM}$ magnesium. 


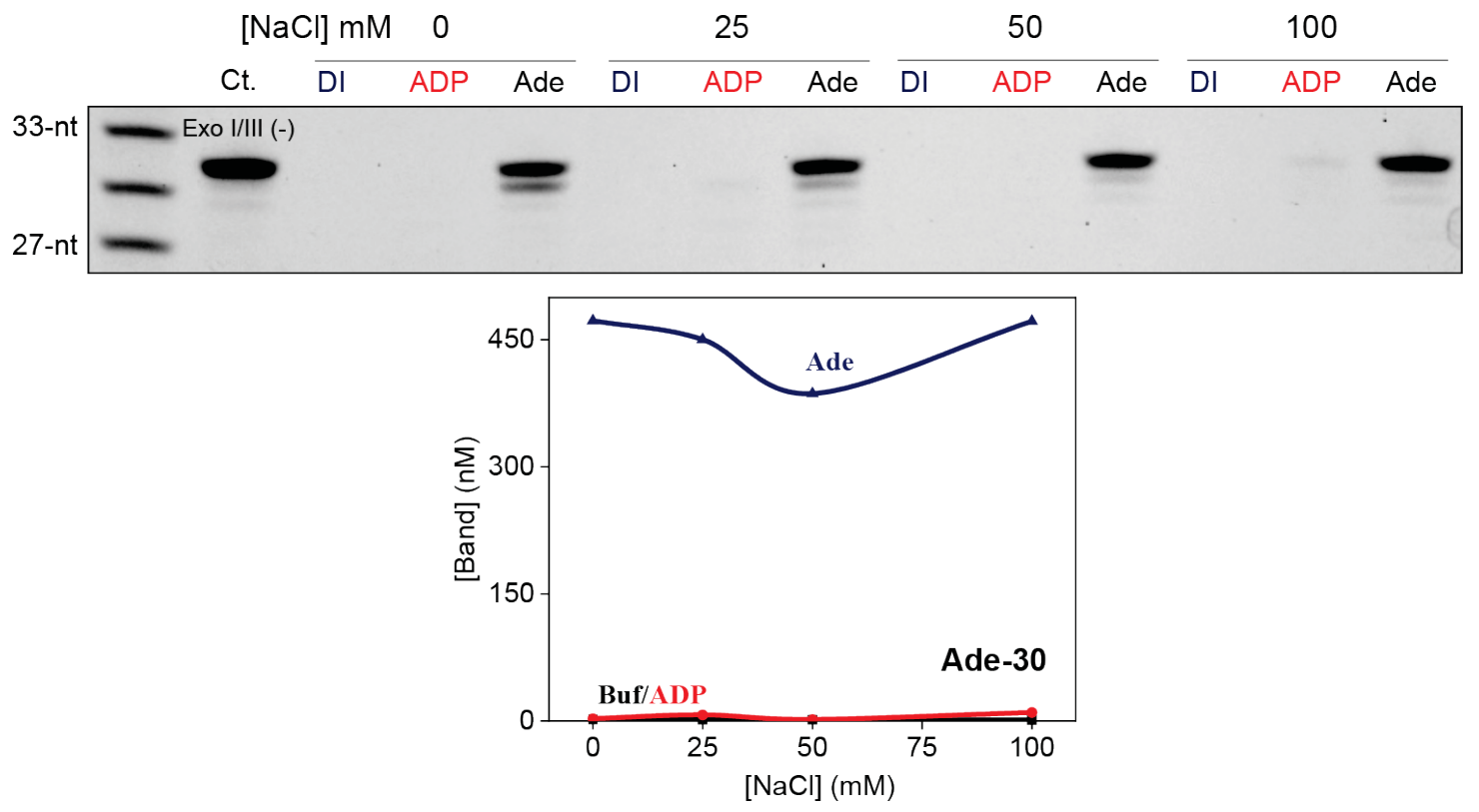

Figure 28. Effect of sodium on target binding.

The binding of adenosine and ADP to the Ade-33 aptamer was independent of sodium concentration. This supports the hypothesis that magnesium is primarily useful as an electrostatic shielding molecule rather than stabilizing the aptamer structure.

\subsubsection{Probing structure-switching functionality with exonuclease I}

Large conformational changes between non-binding and binding competent states in the aptamer structure provide the largest signal gain in electrochemical aptamerbased sensors. ${ }^{48,209}$ While a more stable aptamer structure might have higher binding affinity, it will also produce a large background current because the aptamer will be folded even in the absence of target, bringing the redox probe close to the surface.$^{48}$ In contrast, a destabilized aptamer will favor the non-binding state, reducing the background current and maximizing the signal gain upon target binding. I utilized the enzyme exonuclease I to test the structure-switching functionality of aptamer sequences. The activity of exonuclease I on the targetbound aptamer is inhibited because of a significant change in the conformation of 
the aptamer from single-stranded (enzyme active) conformation to rigid doublestranded structure (enzyme inactive). ${ }^{103,208,210}$ The observed inhibition products of the exonuclease III digestion reaction with the extended ATP-33 and Ade-33 aptamers were ordered from IDT DNA. These fragments are 29- and 30-nt in length, as estimated by utilization of a molecular DNA ladder prepared in-house, and termed ATP-30, Ade-30, and Ade-29. An additional non-binding mutated sequence termed Ade-30m was also ordered with identical sequence but with a single-point mutation that has been shown to inhibit binding. ${ }^{47}$ All sequence structures are shown in figure 29. The cross-reactive ATP-30 and the new adenosine-specific Ade-30 sequences were first incubated with exonuclease I to determine the structure-switching functionality of these truncated aptamers. In the absence of target, exonuclease I can quickly digest the aptamer structure of both the ATP-30 and Ade-30 aptamers, suggesting a single-stranded conformation of these aptamers in the unbound state. Addition of a binding competent target results in inhibition of the enzyme reaction because of formation of doublestranded aptamer-target complexes, which are poor exonuclease I substrates. The ATP-30 sequence exhibited retained products with concentrations of $>500 \mathrm{nM}$ for ATP, ADP and Ade. In contrast, the Ade-30 aptamer only exhibited a retained product with adenosine of $550 \mathrm{nM}$, and no inhibition with ATP or ADP (fig. 29c). 

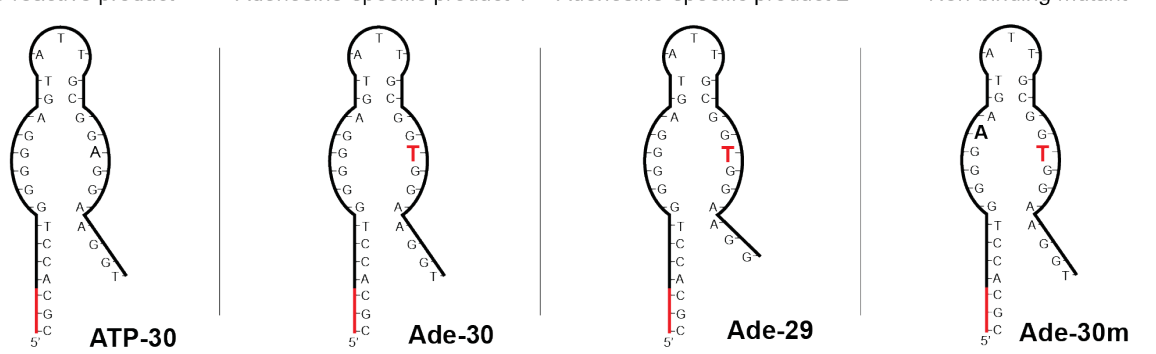

b

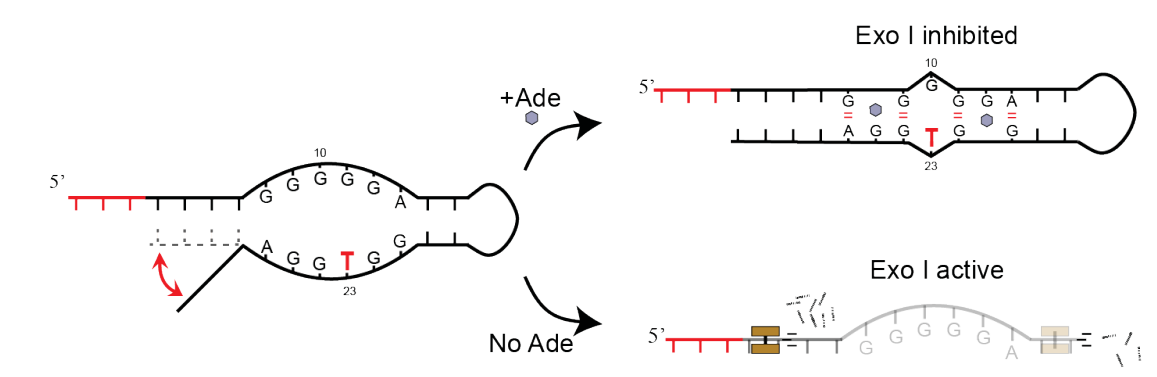

c

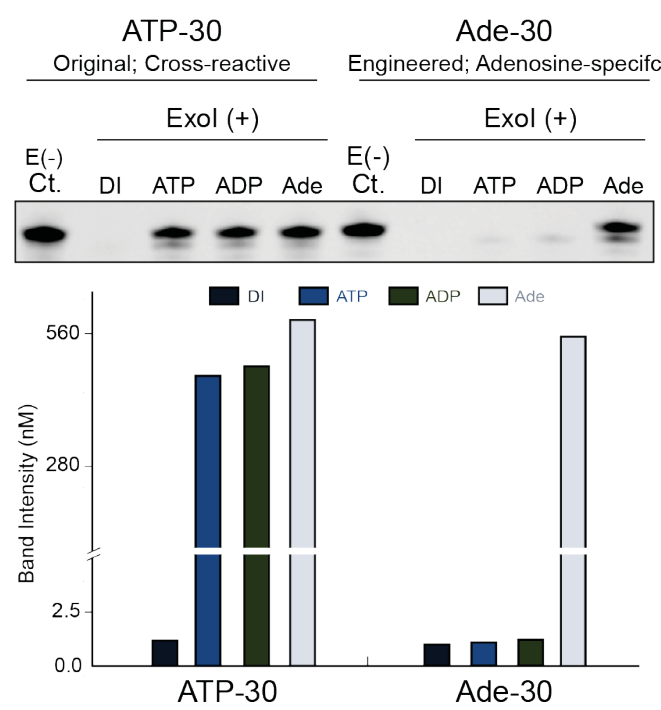

d
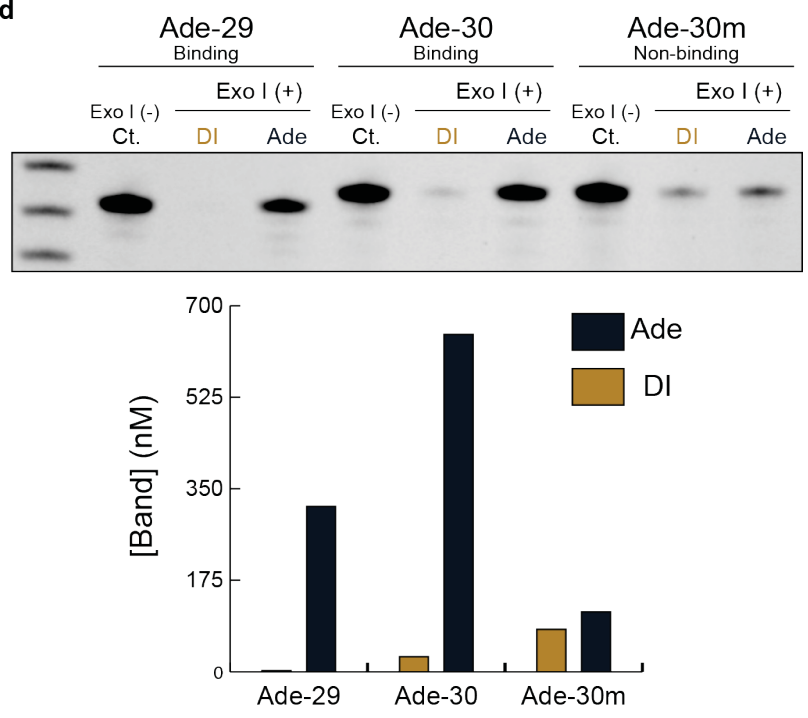

Figure 29. Cross-reactivity testing of a new binding sequence that is specific for adenosine.

(a) Aptamer products of exonuclease III inhibition-based test. The adenosine specific aptamer displayed two distinct products with lengths of 29- and 30-nt. (b) Exonuclease l-based method to identify structure-switching aptamers. The enzyme exonuclease I has a high degree of selectivity for single stranded deoxyribonucleic acid (ssDNA), preferentially hydrolyzing ss-DNA over ds-DNA. ${ }^{103}$ Aptamer-target binding results in structural change of the aptamer conformation from single-stranded DNA to double-stranded DNA, thus inhibiting digestion by exonuclease I. (c) Various targets were incubated with the ATP-30 (original) and 
Ade-30 (engineered) aptamer and Exo I was added. All adenosine phosphate derivatives bound to the cross-reactive ATP-30 aptamer resulting in inhibition products of the enzymatic reaction. The Ade-30 aptamer was only inhibited in the presence of adenosine, exhibiting improved specificity for adenosine compared to ATP and ADP. The inhibition product was plotted as band concentration to compare the apparent binding affinity to various targets. The band intensity (in $\mathrm{nM}$ ) is calculated by comparison to known concentration of control sample andladder.

(d) The two aptamer fragments of 29- and 30-nt, were tested for structureswitching functionality. Both aptamers are digested by exonuclease $I$ in the absence of target, suggesting a mostly single-stranded conformation in the resting state. Upon adenosine binding, the exonuclease I enzyme is inhibited with retained product concentrations of $600 \mathrm{nM}$ and $350 \mathrm{nM}$ for the 30-nt and 29-nt fragments, respectively. A mutant non-binding Ade-30m aptamer did not result in inhibition in the presence or absence of target.

The exonuclease III reaction identifies two aptamer fragments with target-binding induced inhibition with lengths of 29- and 30-nt. Exonuclease I was utilized to test the structure-switching characteristics of these two sequences with adenosine. The Ade-30 aptamer results in adenosine-induced retention of nearly $600 \mathrm{nM}$ of product while the Ade-29 aptamer only has $350 \mathrm{nM}$ (fig. 29d). However, in the absence of adenosine the Ade-30 aptamer exhibits reduced hydrolysis, suggesting intermittent formation of folded structures that are poor substrates for exonuclease I. The Ade-29 aptamer however, is quickly digested in the absence of target, suggesting an equilibrium which favors the unfolded, flexible state in the targetfree conformation (fig. 29d).

\subsubsection{Structure-switching characterization with circular dichroism}

Circular dichroism was utilized to further elucidate the structure-switching properties of the Ade-29 and Ade-30 aptamers. The Ade-29 sequence exhibited peaks at $240 \mathrm{~nm}$ and $265 \mathrm{~nm}$, corresponding to B-form type DNA structures. The 
longer Ade-30 sequence had increased ipeaks at 240 and $265 \mathrm{~nm}$, corresponding to formation of folded DNA (Fig. 30). Addition of adenosine gave a distinct conformational change for the Ade-29 aptamer with increased peak height at 240 and $265 \mathrm{~nm}$ corresponding to ds-DNA conformation. ${ }^{16,211,212}$ In contrast, addition of adenosine to Ade-30 resulted in a less drastic shift in the conformation, possibly because part of the aptamer population is already in a folded conformation (fig. 31). ${ }^{208} \mathrm{An}$ additional control was done with the non-binding sequence Ade- $30 \mathrm{~m}$ to confirm the structure change was caused by target-aptamer binding and not a result of an artificial signal. Addition of adenosine to the Ade- $30 \mathrm{~m}$ sequence did not result in significant changes to the aptamer spectra (Fig. 31).
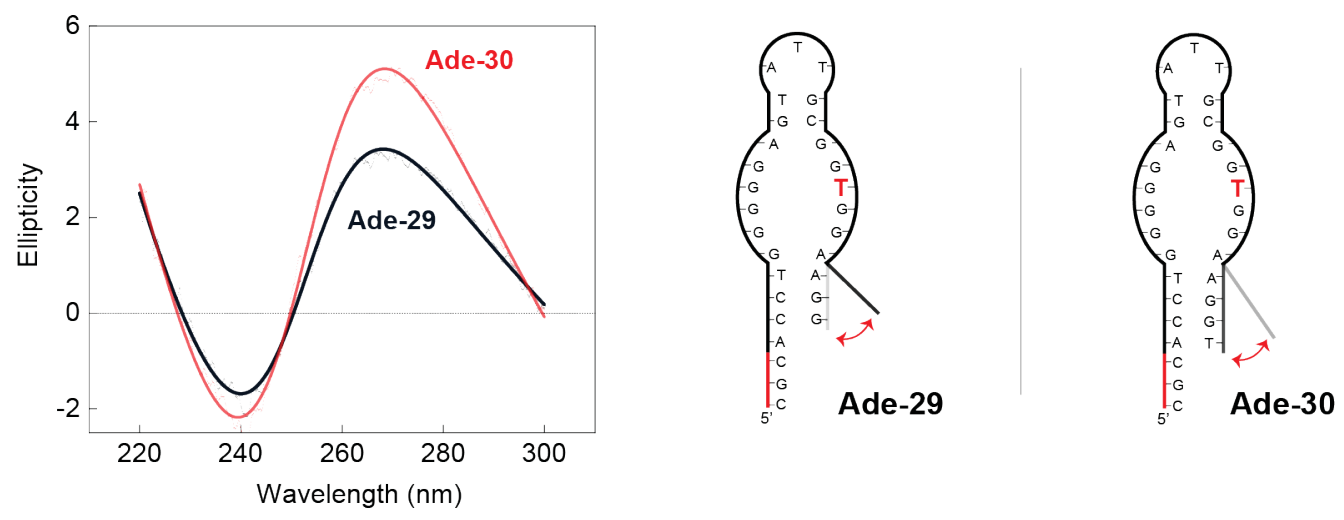

Figure 30. CD spectra for Ade-29 and Ade-30 without addition of target.

The dsDNA character was higher for Ade- 30 which is expected due to its longer stem which stabilizes the stem-loop binding structure. 

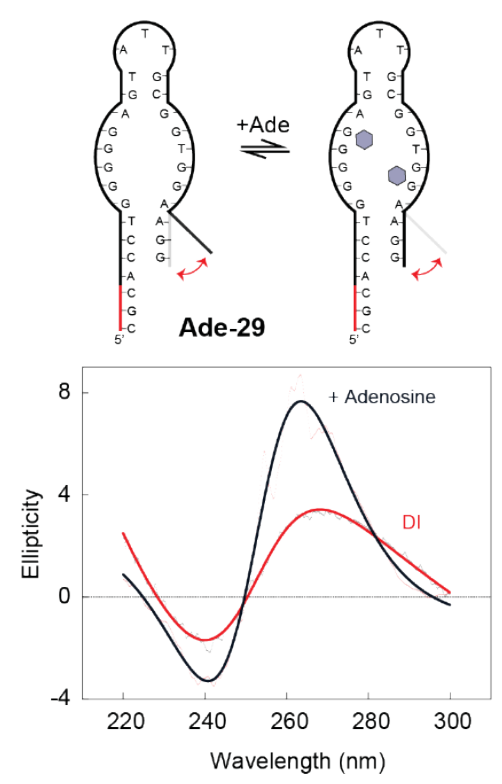
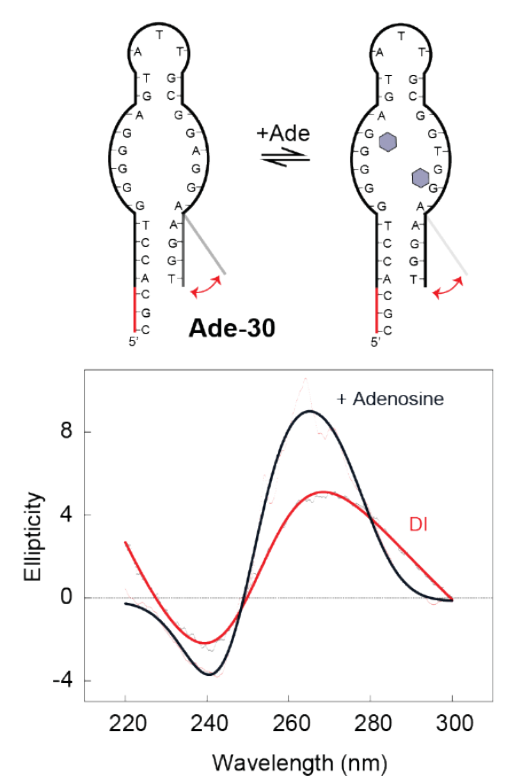
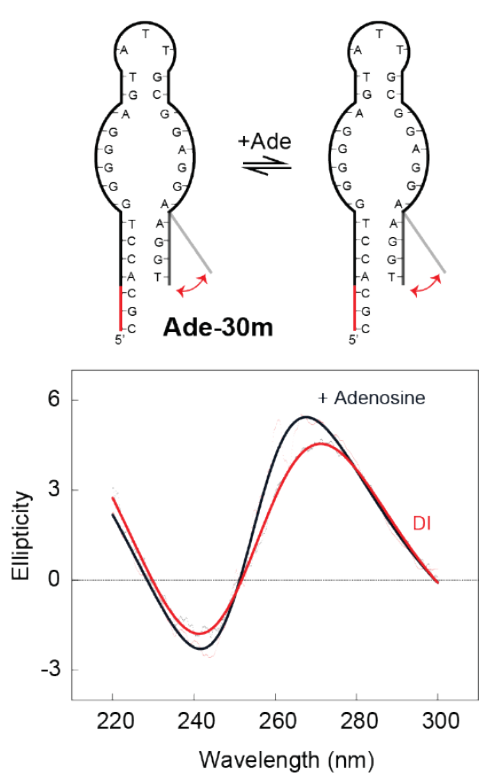

Figure 31. Test of conformational change of Ade-29, Ade-30 and Ade-30m with Circular Dichroism (CD).

The highest degree of conformational change was observed for Ade-29 which agrees with previous reports for an ATP binding aptamer. ${ }^{48} \mathrm{~A}$ control using a nonbinding sequence (Ade-30m) exhibited only a small change upon addition of adenosine which confirms that conformational change occurs only through targetinduced aptamer folding.

\subsubsection{Characterization of aptamer-target binding with ITC}

I utilized isothermal titration calorimetry (ITC) to better understand the binding properties of the aptamer and the effect of magnesium concentration in relation to binding. It has been reported that the original ATP-27 aptamer has two binding sites and weak cooperativity (2 ATP molecules bind per aptamer). ${ }^{197,198}$ Based on these assumptions, we utilized a sequential fitting model to analyze ITC data since the binding sites are different and binding to one site affects the second (i.e., Cooperative). We first tested the binding affinity of the Ade-30 aptamer for the various adenosine derivatives at a low magnesium concentration (1 $\mathrm{mM})$ and identified two adenosine molecules bind per aptamer with negative cooperativity, 
like previously reported studies. ${ }^{110}$ Under these conditions, the binding affinity was strongest for adenosine and weakest for ATP with equilibrium dissociation constants of 8.9, 64 and $115 \mathrm{uM}$ for adenosine, ADP, and ATP, respectively (fig. $32 \mathrm{~b})$ which correlated well with the observed enzymatic results. However, at high magnesium concentration $(10 \mathrm{mM})$ these effects are attenuated with similar binding affinity for Ade, ADP and ATP presumably due to steric shielding by magnesium with equilibrium dissociation constants of 9.5, 18.2, and $27.1 \mathrm{uM}$ for adenosine, ADP and ATP, respectively (Fig. 33). For the cross-reactive ATP-30 aptamer, strong binding of ADP and ATP targets occurred even at magnesium concentrations as low as $0.5 \mathrm{mM}$ with dissociation constants of 2.9 and $5.0 \mathrm{uM}$ for ADP and ATP, respectively (fig. 32b). These results suggest a possible interaction between the adenine at position 23 and the phosphate groups of ADP and ATP for stabilization instead of requirement for magnesium. We presume that the negative charge and size of AMP(-2), ADP (3-) and ATP (4-) play a role in the unfavorable binding for these molecules as compared to adenosine (neutral) at the buffer $\mathrm{pH}$ of 8.5. ${ }^{213}$ Experiments with mutant non-binding aptamer sequences (Ade-30m and ATP-30m) resulted in loss of binding with all the targets (data not shown). ITC and electrophoresis results outline the importance of magnesium to stabilize binding of the ADP and ATP molecules to the engineered adenosine-specific aptamer presumably through a bridging/shielding interaction and the ability of the Ade-30 aptamer to bind adenosine specifically at low magnesium concentrations (fig. 32a). Binding of all targets resulted in a small enthalpy change upon binding of the first target molecule and a larger enthalpy change upon binding of the second target 
molecule. This could be a result of the first Adenosine stabilizing the stem-loop structure through hydrogen bonding with a nucleotide in the aptamer sequence and the second adenosine further stabilizing the rest of the structure resulting in a zipping mechanism of the binding pocket. This correlates well with the entropy change which is large and negative for the second binding event, which is likely from a conformational change in the aptamer structure (fig. 32b). It should be noted that the negative entropy change was smaller for the ATP-30 sequence, suggesting a more stable stem structure prior to binding the second target.

a

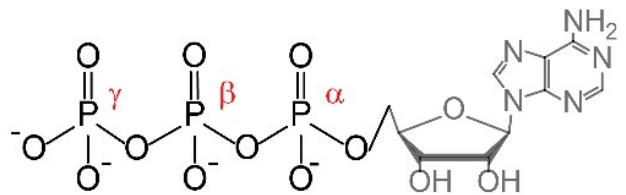
$\ominus$

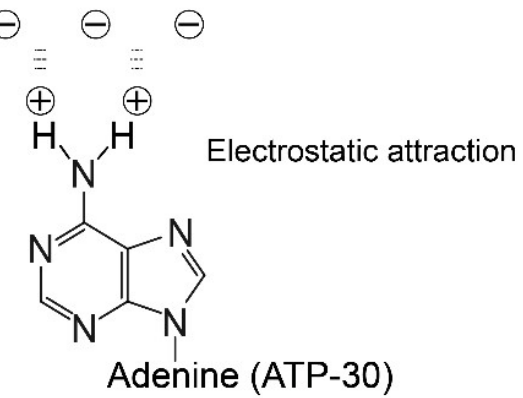

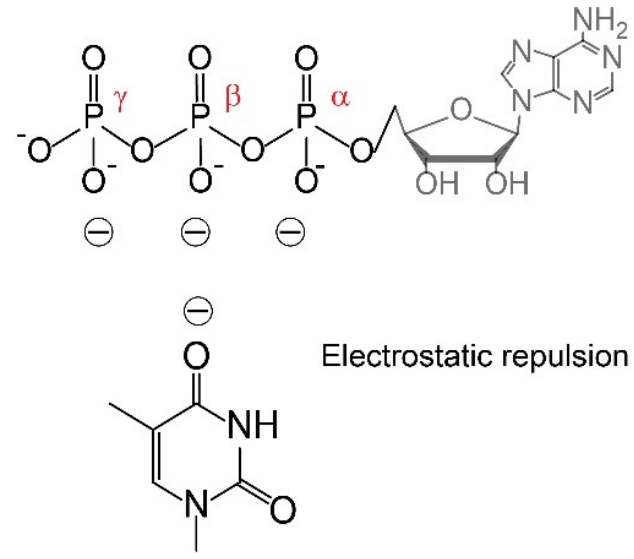

Thymine (Ade-30)

b ITC experimental values for Ade-30 and ATP-30 aptamer sequences.

\begin{tabular}{|c|c|c|c|c|c|c|c|}
\hline & & $\begin{array}{l}K_{d 1} \\
(\mu M)\end{array}$ & $\begin{array}{l}\mathrm{K}_{\mathrm{d} 2} \\
(\mu \mathrm{M})\end{array}$ & $\begin{array}{c}\Delta \mathrm{H}_{1} \\
\text { (cal/mol) }\end{array}$ & $\underset{(\mathrm{cal} / \mathrm{mol})}{\Delta \mathrm{H}_{2}}$ & $\begin{array}{c}\Delta S_{1} \\
\text { (cal/mol/deg) }\end{array}$ & $\begin{array}{c}\Delta \mathrm{S}_{2} \\
\text { (cal/mol/deg) }\end{array}$ \\
\hline \multirow[t]{3}{*}{ Ade $-30^{a}$} & Ade & 8.9 & 35.7 & $-7,743$ & $-2.77 \mathrm{E} 4$ & -3.03 & -73.2 \\
\hline & ADP & 64 & 124 & $-6,269$ & $-3.42 E 4$ & -1.97 & -97.6 \\
\hline & ATP & 115 & 205 & $-4,671$ & $-3.00 \mathrm{E} 4$ & 2.26 & -84.5 \\
\hline \multirow[t]{2}{*}{ ATP-30 } & ADP & 2.9 & 40.0 & $-6,355$ & $-2.20 \mathrm{E} 4$ & 3.87 & -54.1 \\
\hline & ATP & 5.0 & 52.0 & $-4,203$ & $-1.85 E 4$ & 10.1 & -42.8 \\
\hline
\end{tabular}

(a) Experiments performed at $1 \mathrm{mM} \mathrm{MgCl}_{2}$ (b) Experiments performed at $0.5 \mathrm{mM} \mathrm{MgCl} 2$

Figure 32. Proposed mechanism of Ade-30 specificity for Adenosine due to steric hinderance with ATP and ADP at low magnesium concentration. (table 1) ITC binding affinity measurements for adenosine, ADP, and ATP. The 
buffer conditions were chosen to match the enzyme-based conditions. (10 mM BTP, $0.5-1 \mathrm{mM} \mathrm{MgCl} 2$ at $\mathrm{pH}$ 8.5). The DNA concentration in the cell was $20 \mu \mathrm{M}$ and the concentration of small molecule in the syringe was $650 \mu \mathrm{M}$. A sequential binding model was used to fit and analyze the binding curves.
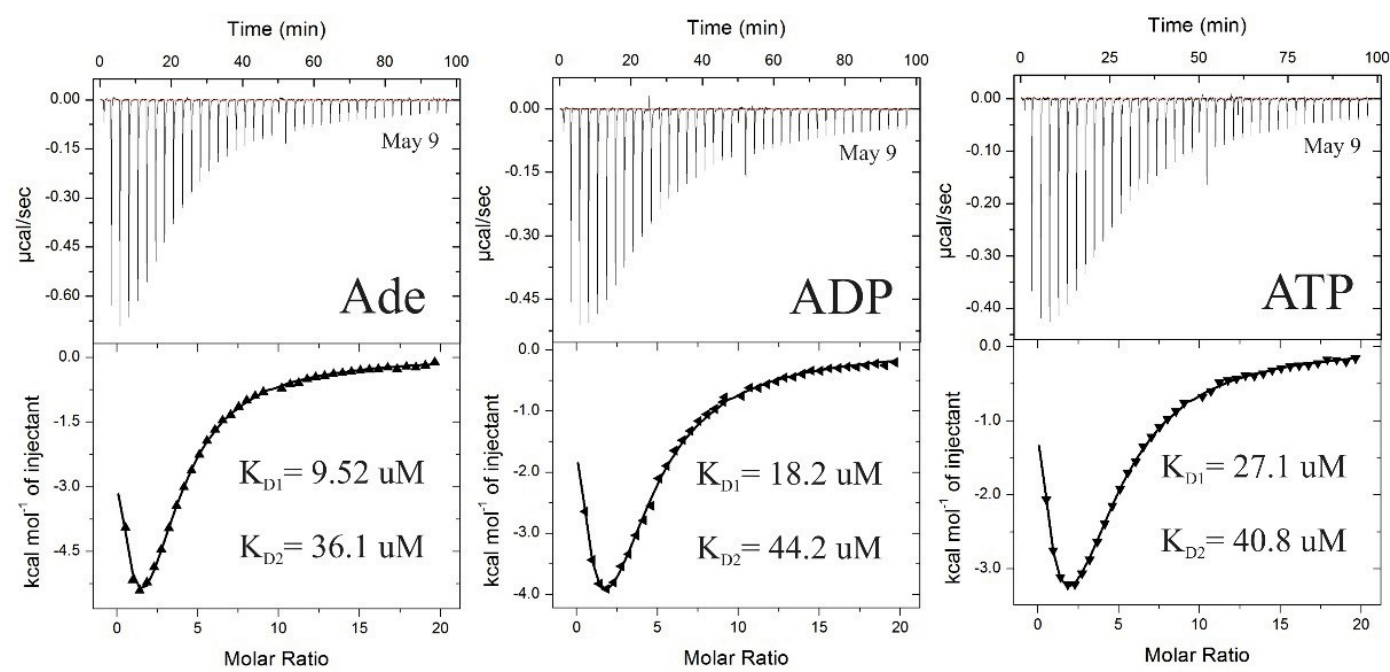

Figure 33. ITC binding experiments at $10 \mathrm{mM}$ magnesium for the Ade-30 sequence.

Under conditions of high magnesium there is significant binding for all targets although it appears ATP has weaker binding. This is presumably due to the steric shielding effect of magnesium.

The Ade-29 aptamer displayed strong binding affinity for adenosine with a dissociation constant of 20,131 and $173 \mu \mathrm{M}$ for adenosine, ADP and ATP, respectively (fig. 34). The measured affinity was 2-fold higher for Ade-30 as compared to Ade-29. This is consistent with the fact that Ade-29 has an energy penalty to reach a binding competent state. We chose the Ade-29 aptamer to design a redox-modified aptamer for electrochemical sensing since it could bind to adenosine with relatively high affinity and maximize the signal change upon target binding with a large change in structure conformation. 

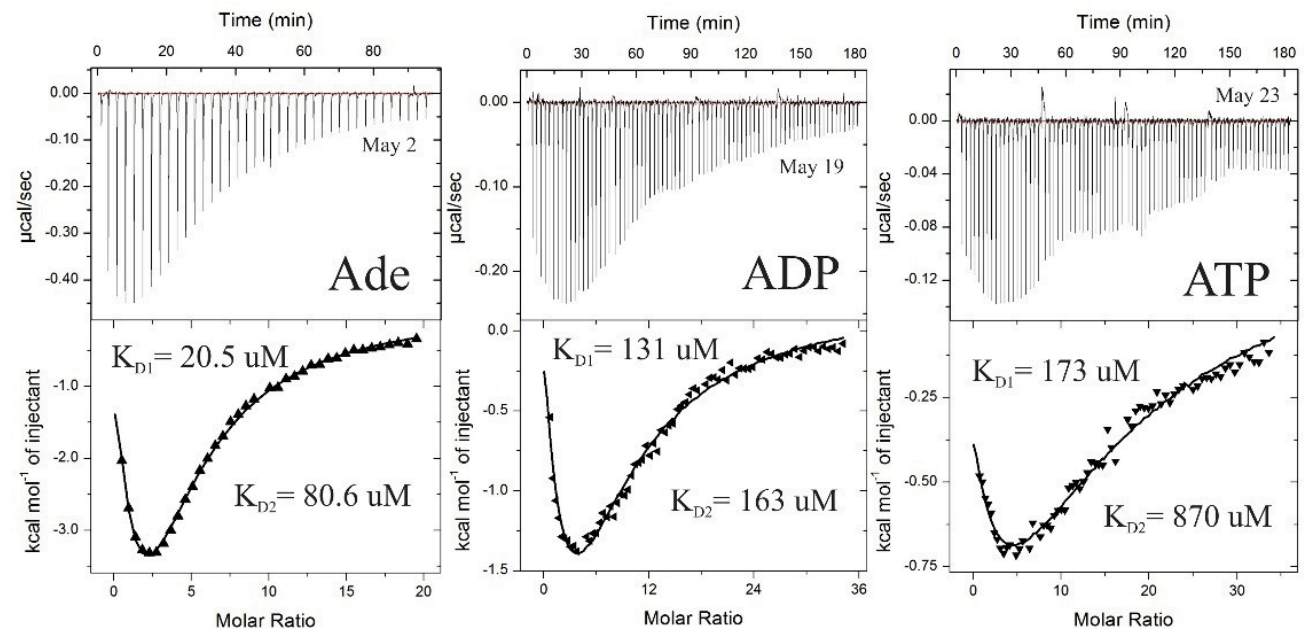

Figure 34. Binding affinity of Ade-29 to adenosine, ADP and ATP under optimal binding conditions (10 mM BTP, $1 \mathrm{mM}$ magnesium at $\mathrm{pH} 8.5$ ).

The binding affinity was reduced by 2 -fold for all targets when compared to the Ade-30 aptamer.

\subsubsection{Design of methylene-blue modified aptamer sequence (Ade-29-MB)}

In blood or serum, spectroscopic methods are seldom utilized because of significant interference from the matrix complicating sensitive measurements in undiluted samples. ${ }^{176,193}$ Therefore, I adapted the Ade-29 aptamer for electrochemical sensing which is both sensitive and highly selective, readily applicable for detection in undiluted complex samples. ${ }^{10}$ Electrochemical aptamerbased signaling is a result of specific target-induced changes in the conformation of an aptamer immobilized to an electrode surface and covalently labeled with an electroactive molecule, producing a current change that corresponds to target concentration. ${ }^{192,214,215}$ The redox molecule methylene blue was covalentlyattached to the 3' end of the Ade-29 aptamer while the 5' end was chemically modified with an alkane thiol. To ensure close contact between the methylene blue and electrode surface, the Ade-29-MB aptamer was modified with three additional 
thymine residues at the $3^{\prime}$ end to match the non-binding residues at the $5^{\prime}$ end that are conserved from the previous enzymatic experiments (fig. 35). In the absence of target, the Ade-29-MB structure is flexible, placing the redox tag away from the electrode and inhibiting electron transport. Target-induced changes in the structure result in folding of the aptamer, bringing the redox tag in proximity to the electrode surface and increasing the measured current. The advantages of this platform is that it enables sensitive sensors with large signal gain, provides a large degree of selectivity because only the aptamer redox element is active in the potential window employed and is indifferent to non-specific binders or contaminants that might absorb on the electrode surface. ${ }^{181,215}$

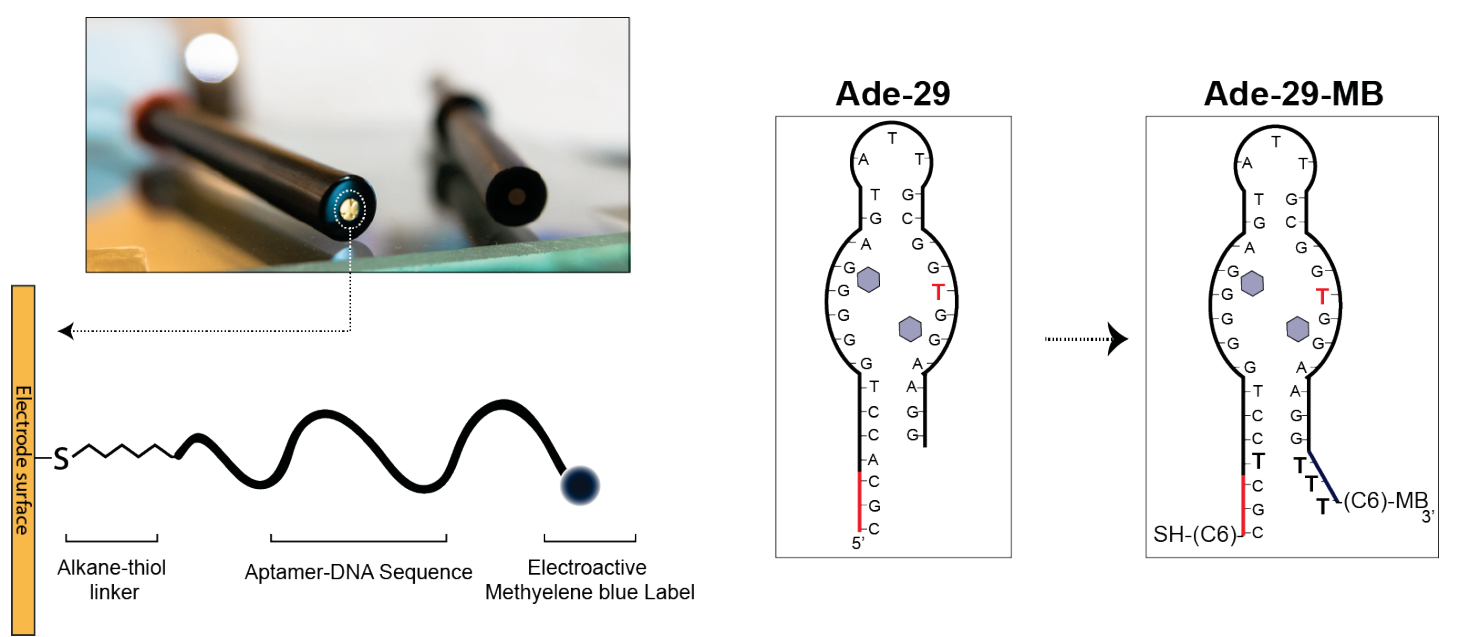

Figure 35. Design of methylene blue modified adenosine aptamer sequence: Ade29-MB.

The aptamer is functionalized with a thiol group on the 5 ' end to immobilize onto a gold electrode surface through strong thiol-gold bonds. The 3' end of the aptamer is functionalized with the electroactive molecule methylene blue (MB). The 5' extension of the aptamer is kept intact from the enzyme-selected structure and three thymine non-binding nucleotides are added to the 3 ' end to match the number of nucleotides on the 5' end, allowing close contact between the MB and the electrode surface upon target-induced folding of the aptamer structure. 


\subsubsection{Electrode surface modification and effects on signaling}

Initially, we prepared the Ade-29-MB-based sensor using a previously reported method of surface modification with 6-mercapto-1-hexanol (MCH). ${ }^{94} \mathrm{MCH}$ can remove non-specifically bound aptamer molecules from the gold surface and lead to a more organized monolayer and large population of "active-sensing" aptamers. ${ }^{95}$ The results were promising, with addition of adenosine leading to a large positive change in the current while addition of ADP and ATP did not change the current (Fig. 36). The sensor was slightly cross-reactive for ATP and ADP when performing detection in a buffer with $1 \mathrm{mM}$ magnesium, which could be a result of the higher local magnesium concentration at the electrode surface and nonspecific folding of the aptamer even in the absence of the target. ${ }^{195}$ Therefore, we performed the rest of the testing in buffer without any magnesium which resulted in minimal cross-reactivity (Fig. 36). Sodium was added to aid in conductivity of the solution but it did not cause any effect on target binding (fig. 37).
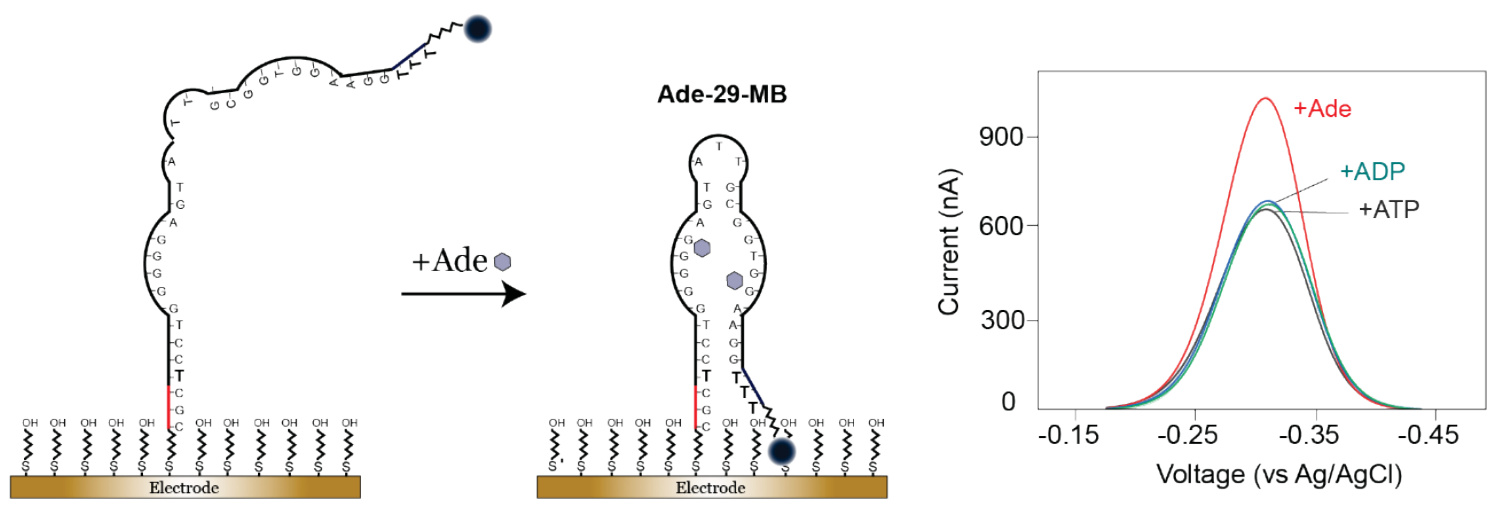

Figure 36. Platform for electrochemical sensing of adenosine using the adenosine specific Ade-29-MB aptamer.

(a) In the absence of adenosine, the redox probe is far from the electrode surface, thus only a small current is produced through the flexibility of the probe whichcan 
strike the surface at a slow rate. A target-induced change in the aptamer conformation results in folding of the aptamer, bringing the redox tag into proximity of the electrode surface and increasing the rate of electron transport and thus the measured current. There is an equilibrium between the non-binding state (ss-DNA) and the binding competent state which directly affects the binding affinity and the background current. (right) Output current from addition of different targets ([target] $=250 \mu \mathrm{M}$ ) as detected by Ade-29-MB. Only specific binding of adenosine results in an increase in the measured current.
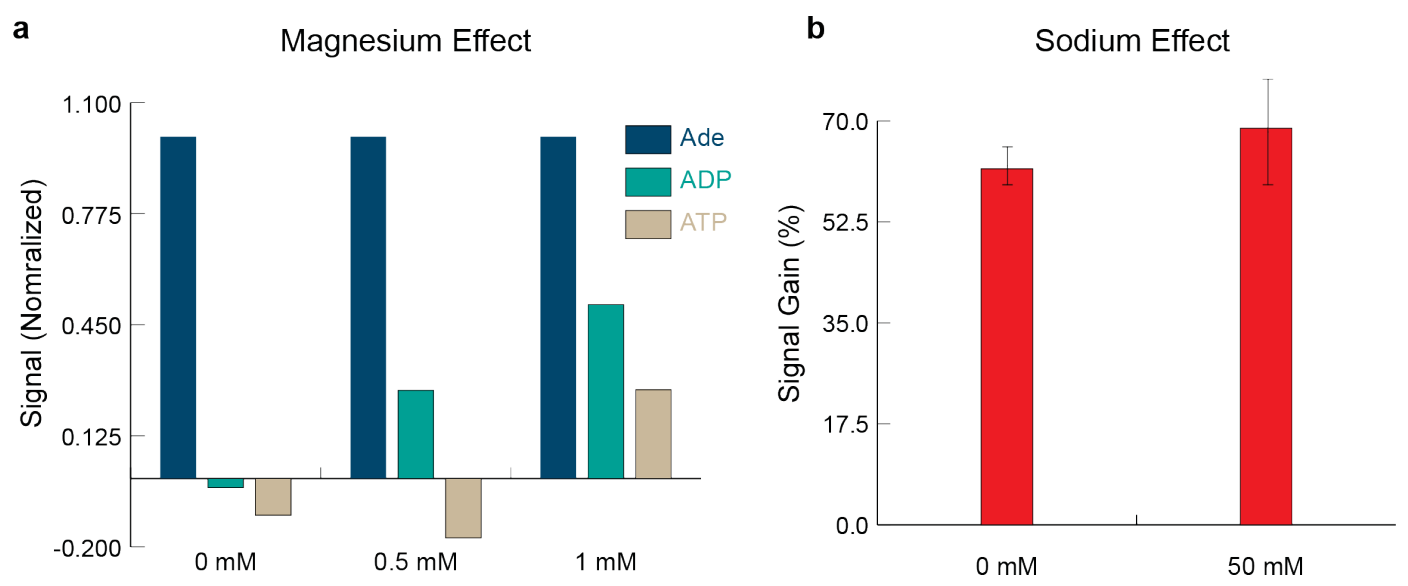

Figure 37. Magnesium and sodium effect on electrochemical detection.

Aptamer specificity is reduced in electrochemical measurements in the presence of $1 \mathrm{mM}$ magnesium. This is likely a result of increased local concentrations of magnesium at the electrode surface, which is negatively charged. The specificity can be regained by lowering the magnesium concentration in the buffer. Sodium did not affect the electrochemical signaling.

Aiming to increase the signal gain of the sensor, we tested a combination of $\mathrm{MCH}$ and dithiothreitol (DTT) as backfillers, with DTT being able to fill most pinholes in the monolayer structure reducing noise and displacing non-specifically bound nucleotides as well as increasing inter-aptamer distances which could aid in target binding. ${ }^{98,216}$ Interestingly, we observed higher signal gain with a combination of $\mathrm{MCH} / \mathrm{DTT}$ as compared to $\mathrm{MCH}$ or DTT alone (Fig. 38a). The higher signal gain resulted in better performance at low concentrations of adenosine, enhancing the 
sensor sensitivity (fig. 38b). This is a result from lower background current as well as higher signal gain upon target binding which could be explained by better monolayer stability, reduced non-specific interactions between the aptamer bases and the electrode surface and better aptamer spacing on the gold surface. ${ }^{216}$ Dithiothreitol (DTT) alone significantly increased the electron transfer rate similarly as to what was previously observed by using short alkyl-chain backfillers, but resulted in lower signal gains because of increased background current. ${ }^{97}$ The aptamer surface coverage on the gold electrode was similar for $\mathrm{MCH}, \mathrm{MCH} / \mathrm{DTT}$ and DTT, therefore it is unlikely that the signal enhancement was a result of more probes being present on the surface (Fig. 38c). Signal response and equilibration times were also improved with the mixed monolayer of MCH/DTT as compared to $\mathrm{MCH}$ (data not shown). The amplitude was also optimized, with $25 \mathrm{mV}$ resulting in the best signal gain (fig. 38d). It is possible that the mixed monolayer could be generally applied to other aptamer sensors but further investigation is required to understand whether this effect is specific for the Ade-29-MB aptamer. 
a

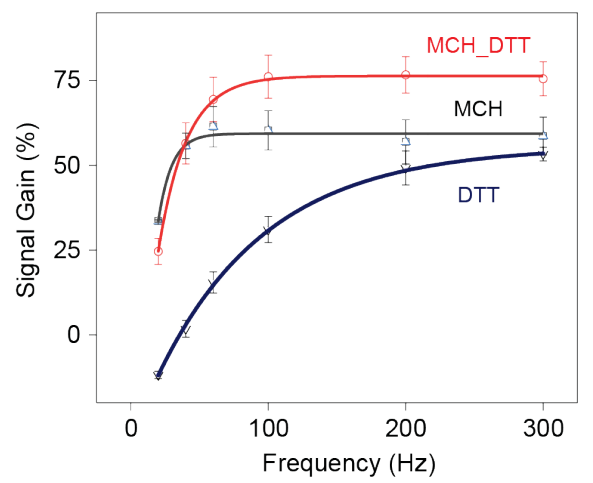

C

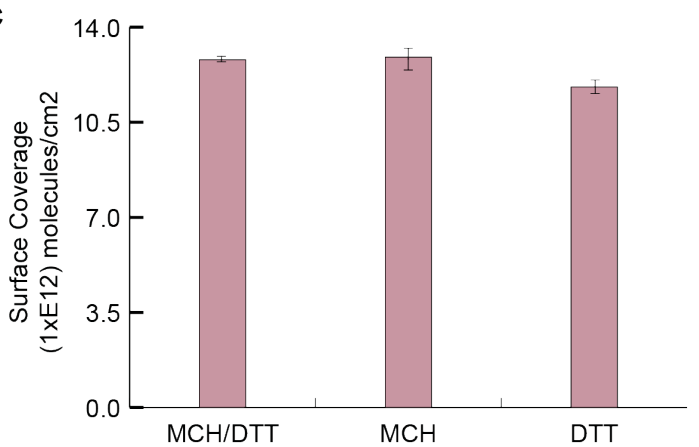

b

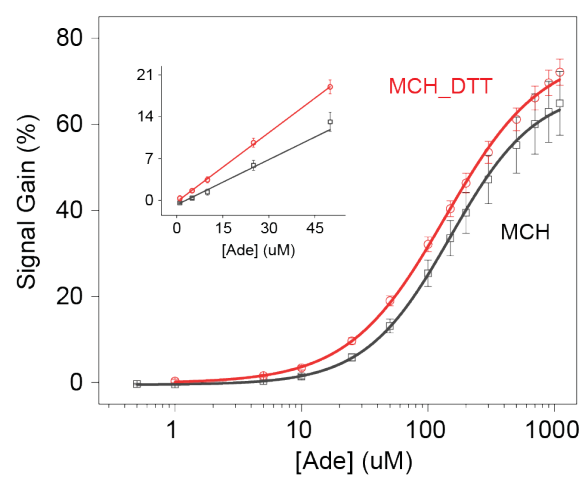

d

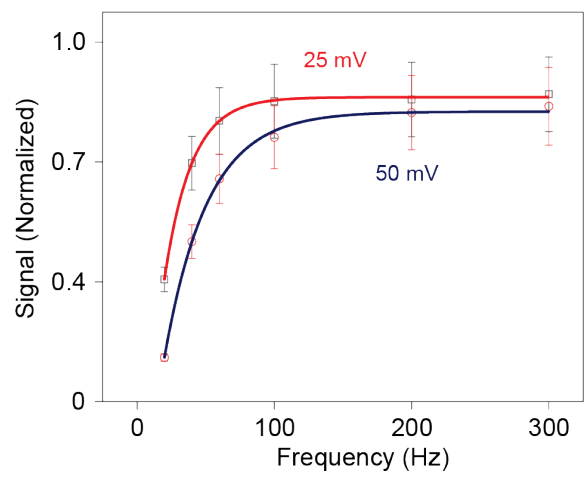

Figure 38. $\mathrm{MCH}$ and DTT effect on electrochemical sensor and optimization. Various backfiller molecules were utilized to stabilize the gold-aptamer monolayer. (a) A combination of MCH/DTT resulted in the highest signal gain sensors at all frequencies tested. This is likely a result of better inter-aptamer spacing and lower non-specific adsorption of molecules to the electrode surface. (b) The MCH/DTT combination has better analytical performance, specially at lower adenosine concentrations, with higher apparent binding affinity. (c) The surface coverage was unchanged regardless of the backfilling molecule. This suggest that any improvement in the signaling is not a result of number of DNA aptamers immobilized on the surface of the electrode. (d) The amplitude of MCH/DTT modified electrodes was optimal at $25 \mathrm{mV}$ at all frequencies.

\subsubsection{Detection of adenosine in undiluted and unprocessed serum}

Calibration curves were collected by titrating targets into a 4-mL electrochemical cell (Fig. 39). A hill binding model was utilized for fitting the titration curves which resulted in an apparent binding affinity slightly lower than ITC likely because detection was performed in buffer without magnesium and the surface 
immobilization often affects affinity. We observed a weak cooperativity from the fitting which corresponds well with other reported methods and our own ITC data. Signal saturation was reached around $1 \mathrm{mM}$ with a signal maximum of $120 \%$ and with a linear range from 1-50 uM in buffer (Fig. 39a and b). The frequency was reoptimized for detection in whole serum to account for the change in viscosity of the solution which has previously been reported to slow the rate of electron trasnfer. ${ }^{192}$ However, the results indicated that the optimal frequency was also $100 \mathrm{~Hz}$. in whole serum (Fig. 39a). We could perform detection immediately after target addition and observed complete signal saturation after 30 seconds (Fig. 39c). Signal saturation was also around $1 \mathrm{mM}$ in undiluted serum with a linear range from 1-100 uM (fig. 39c). Detection in serum resulted in reduced signal gain because of the endogenous concentration of magnesium and other ions. The magnesium concentration in serum is reported to be between $1-4 \mathrm{mM}$. This produces a higher background current presumably because of stabilization of the binding competent state in the absence of target accounting for about $20 \%$ signal loss (Fig. 40c). The rest of the difference is attributed to the viscosity of the serum and possible degradation of the reference electrode. Measurements in undiluted serum also present higher cross-reactivity because of the endogenous magnesium concentration (Fig. 40c). Simply adding EDTA (2.5 mM) to the detection solution to quench the endogenous magnesium is sufficient to regain the desired specificity (Fig. 40c). Under such conditions, adenosine detection is specific compared to ATP, ADP, AMP and GTP (fig. 41) even under significant differences in the concentration of the targets. 

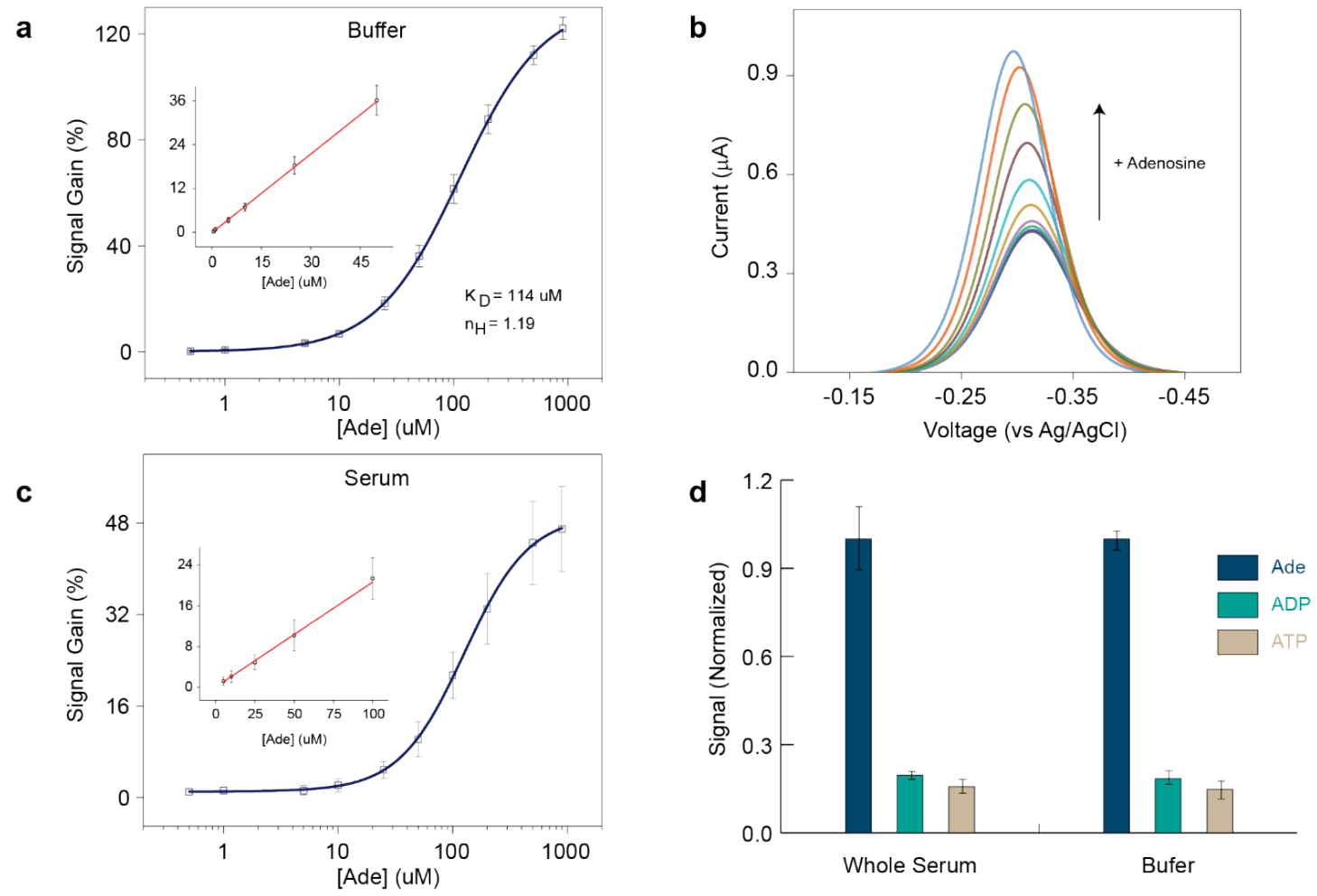

Figure 39. Electrochemical detection of adenosine in buffer and serum. (a) Calibration curve of adenosine in buffer with linear range (inset) between 1 $50 \mu \mathrm{M}$ and a maximum signal gain of $120 \%$ (b) Raw current response from SWV experiment with increasing concentrations of adenosine. (c) Calibration curve for adenosine in undiluted serum spiked with increasing concentrations of adenosine reported as signal gain percentage with linear range between $1-50 \mu \mathrm{M}$ (inset). (d) Comparison of normalized signal gain and specificity of adenosine over ATP and ADP in buffer and undiluted serum with $2.5 \mathrm{mM}$ EDTA and $500 \mu \mathrm{M}$ of each target.
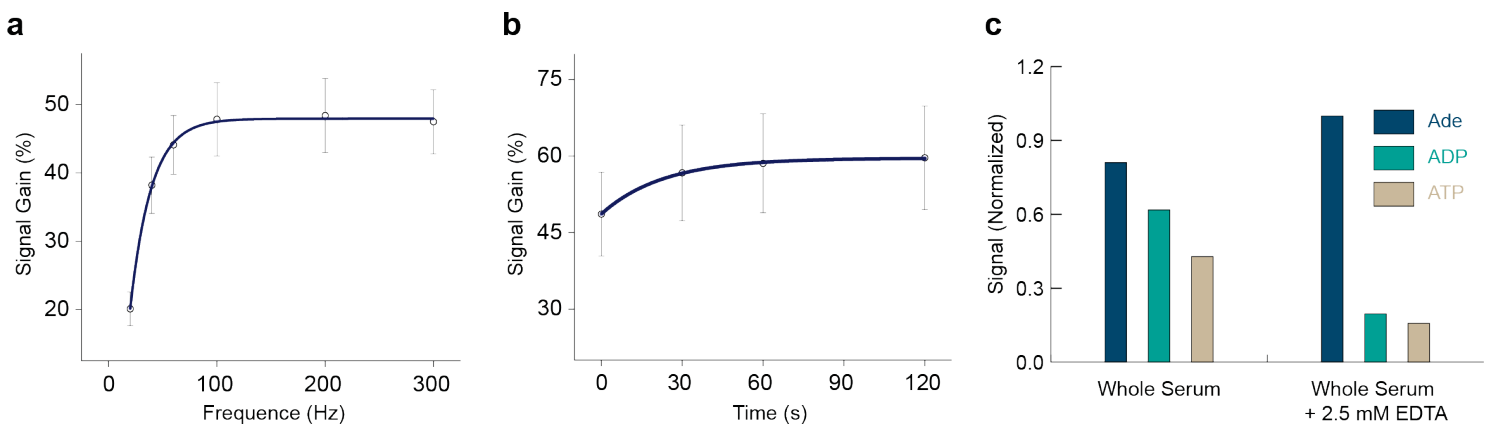

Figure 40. Serum optimization and cross-reactivity in undiluted serum.

(a) Frequency optimization in whole serum exhibited an optimal of $100 \mathrm{~Hz}$. which was utilized for all other experiments. (b) Signal gain over time in undiluted serum. 
Adenosine detection can be performed immediately after target addition and with signal saturation within 30 seconds. (c) Cross-reactivity in serum presumably because of endogenous magnesium and other divalent ions. EDTA can be added to quench the free divalent ions in undiluted serum to regain the desired specificity. $[$ Target $]=500 \mu \mathrm{M}$.

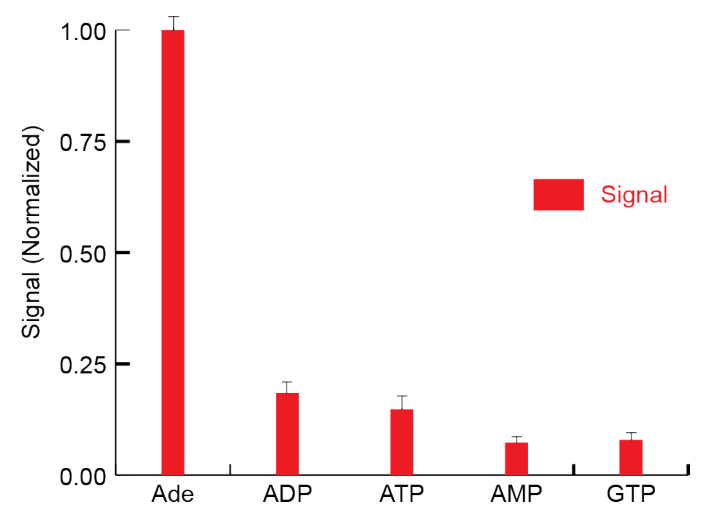

Figure 41. Adenosine specific detection and cross-reactivity with ATP, ADP, AMP and GTP.

The Ade-29-MB aptamer is highly specific for adenosine when compared to ATP, ADP, AMP and GTP. AMP has even lower interaction with the aptamer, likely due to strong repulsion between the alpha phosphate and the thymine at position 23 . $[$ Target $]=250 \mathrm{uM}$

\subsection{Conclusion}

Initially, we hypothesized that nucleotide substitutions near the binding site would modulate the aptamer specificity. Indeed, our new adenosine aptamer varies at a single nucleotide and displays over 10-fold higher affinity for adenosine compared to ATP. The sensor was utilized to measure adenosine in undiluted serum with nearly no response for ATP or ADP, demonstrating its potential use in medical settings with similar performance as compared to buffer. The Ade-29 aptamer exhibits increased specificity for adenosine while retaining the same stem-loop structure of the original aptamer. The ability to use the same aptamer stem-loop structure is valuable as many methods have been developed for this structure and 
sufficient characterization has been published, opening the door for further increasing the sensitivity of the sensor with adaptation to different signaling mechanisms. For example, graphene-based methods can be readily used with our structure-switching aptamer. The potential applications for this sensor will be in directly measuring adenosine in blood/serum samples to diagnose potential diseases, as a research lab tool in studies of the role of adenosine in various diseases, and potential identification of new therapies where adenosine concentration is important. We also envision the use of this sensor to indirectly measure the activity of adenosine regulating enzymes such as adenosine deaminase (ADA), adenosine kinase (ADK) and S-adenosylhomocysteine hydrolase (SAHH) and profiling of potential inhibitors of these enzymes. All of this is a direct result of the ability of the aptamer to measure adenosine in the presence of ATP, ADP, and AMP, which are the major sources of adenosine. Limitations of the newly identified Ade-29 aptamer include the increased cross-reactivity in the presence of high concentrations of magnesium which likely prohibits its use in continuous detection for neuromodulator release measurements in its current state and its limited sensitivity. We believe these obstacles can be overcome with further engineering of the aptamer sequence, improvements to the electrochemical detection method and adaption to different read-out methodologies. 


\section{CHAPTER 5}

\section{Nuclease-guided truncation of aptamers for optimal structure-switching functionality and application in electrochemical sensors}

Aptamers which do not undergo conformational changes upon target-binding cannot be easily incorporated into signal transduction mechanisms. Conventional methods to instill structure-switching functionality -to otherwise conformationally static aptamers- often involve meticulous and labor-intensive testing of various 'pilot' sequences. To enhance, facilitate, expedite and lower the cost of finding optimal structure-switching aptamers, we employ a T5-exonuclease guided method that truncates conformationally static aptamers, yielding aptamer sequences with large difference in the target-bound and un-bound states, with target-induced change in the conformation. The method was demonstrated to generate aptamer sequences with target-induced structure-switching functionality for the schedule I drug methylenedioxypyrovalerone (MDPV) and medical biomarkers deoxycorticosterone 21-glucoside (DOG) and adenosine triphosphate (ATP). Incredibly, identification of an optimal truncated aptamer can be achieved in a single experiment, from a single aptamer parent sequence. Additionally, DNA without expensive purification can be utilized, providing a low-cost alternative to generate optimal structure-switching aptamers. The method provides a key tool in development of E-AB sensors by lowering the cost, development time and complexity of aptamer engineering for electrochemical transduction. 


\subsection{Introduction}

Electrochemical aptamer-based (E-AB) sensors are becoming increasingly popular for detection of various targets in complex sample matrices (e.g., human serum and adulterated drugs). ${ }^{10,90}$ Functionality in complex samples is provided by the incredible specificity of aptamers, which can recognize even small changes in the structure of a molecule, and the high selectivity of electrochemistry because of the paucity of electroactive species in most samples. ${ }^{194}$ The success of these sensors largely lies in the ability to couple the binding reaction -between analyte and aptamer- into a measurable electronic signal (i.e., current). Electrochemical signaling occurs via a target-induced conformational changes in the aptamer structure, which modulates the rate of electron transfer between an electroactive molecule (covalently modified onto the aptamer) and the immobilizing electrode surface (Scheme 1). ${ }^{215}$ Transfer of electrons (i.e., signal-output) is determined by the distance of the redox-tag from the electrode surface and flexibility of the aptamer structure -both phenomena are directly affected by the aptamer secondary structure (i.e., conformation). ${ }^{92}$

Aptamer selection strategies often isolate aptamers with relatively fixed (i.e., semipermanent) conformations, which do not undergo target-induced structureswitching. Therefore, development of E-AB sensors requires meticulous and expensive aptamer engineering to impart structure-switching functionality to otherwise highly stable (i.e., folded) aptamers. ${ }^{48,110}$ The most important factor for optimizing the signal gain in $\mathrm{E}-\mathrm{AB}$ sensors is finding the correct aptamer sequence which can 'switch' between distinct conformations before and after target binding. 


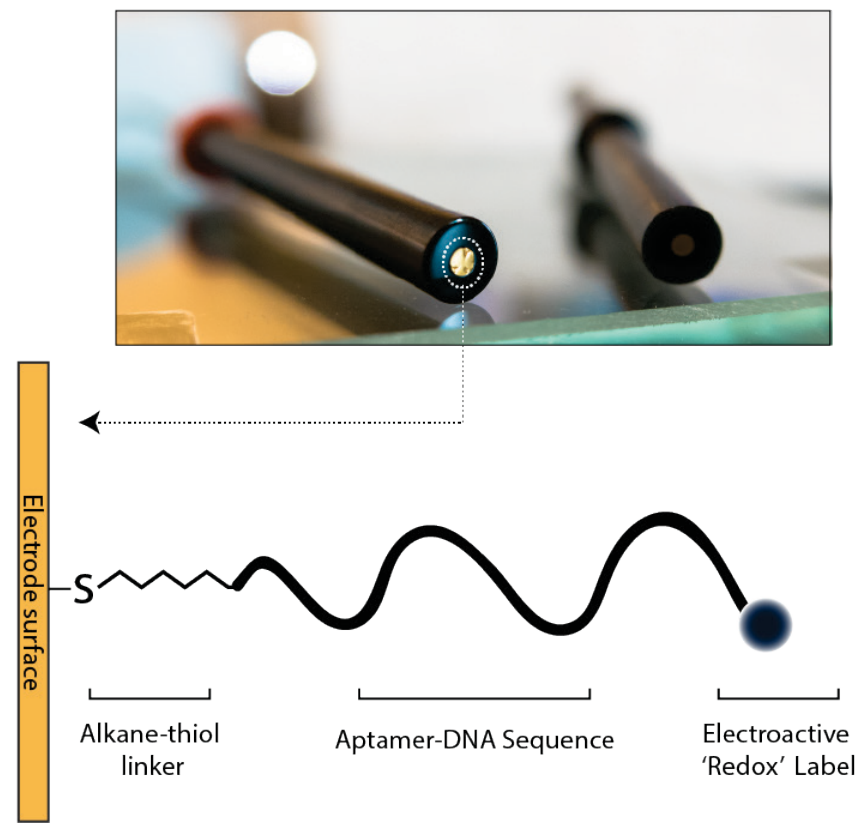

Scheme 1. Electrochemical aptamer-based (E-AB) sensor design.

Aptamers can be chemically functionalized with an alkane-thiol group for immobilization onto electrode surfaces by strong thiol-gold bonds, preventing desorption of aptamers during fabrication and measurements. The short alkanethiol functional group also provides stabilization during formation of the aptamer monolayer by hydrophobic interactions with a diluent alkane-thiol molecule (omitted for clarity, see chapter 4 for detailed structure). The opposite end of the aptamer is chemically modified with an electroactive molecule such as methylene blue. The aptamer functions as a target-sensitive 'switch', separating the electroactive molecule from the electrode surface and thus modulating the measured current as a function of analyte concentration.

A method that can add structure-switching functionality to the currently available and extensive aptamer library is highly desirable. While aptamer isolation methods for direct selection of structure-switching aptamers have been previously reported with limited examples and applications, adapting the already available and extensive library would be immediately impactful. ${ }^{217}$ The main advantages of structure-switching aptamers are their remarkable specificity (since weak-binding and non-binding molecules don't typically induce structure-switching) and their 
compatibility with various transduction methods such as fluorescence, colorchange and electrochemistry. Generally, conventionally isolated DNA aptamers form stable, folded structures with distinct geometries such as stem-loops and three-way junctions; in both cases, intramolecular helices stabilize formation of a binding pocket, such as a three-way junction (three stems stabilize a central binding pocket). Prediction of the lowest energy conformation is based on calculations of the energy stabilization of individual matched base-pairs and energy penalties of loops, non-binding nucleotides or mismatched base pairs. ${ }^{218}$ Increasing the number of matched base-pairs shifts the conformational equilibrium towards a folded conformation while removal of stabilizing base-pairs or addition of mismatched base-pairs would destabilize the structure until an unfolded structure is favored (Scheme 2).

Stem-length effect on aptamer conformation
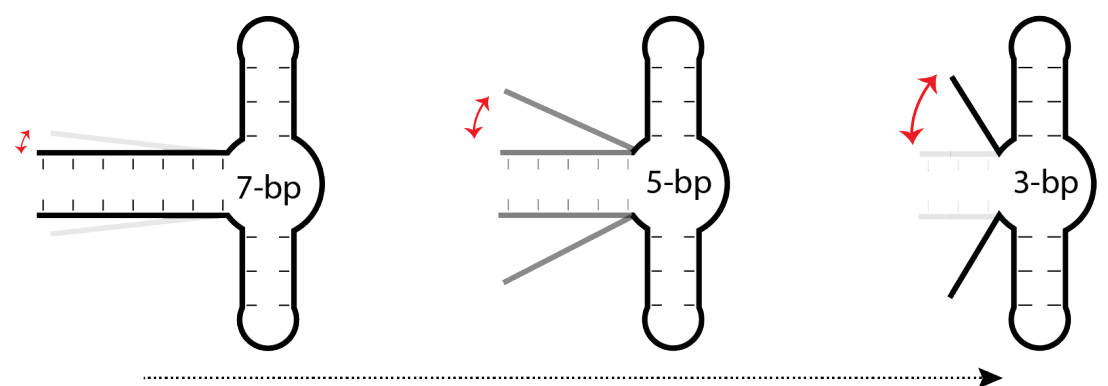

Increasing structure-switching

Scheme 2. Stem-length effect on aptamer conformation.

Aptamer structures with long stems are highly stable and almost completely folded into organized and rigid double-stranded structures with little conformation difference upon target-binding. As the number of stabilizing base-pairs in the stem is reduced, the aptamer is destabilized and unfolds into a disorganized and flexible structure of single-stranded DNA. A minimum number of base-pairs is required for target-binding, with conventional methods requiring complex and costly aptamer 
re-engineering to discover the correct structure-switching sequence and structure.

Several strategies have been demonstrated to instill structure-switching functionality to aptamers including: (a) destabilization by trial-and-error truncation or mutation of the native aptamer structure such as removing base pairs or adding mismatched nucleotides, (b) separation of the folded binding pocket by addition of a polymer linker or long sequences of non-binding nucleotides and (c) addition of a competing oligonucleotide strand that will hybridize with the aptamer, block the binding site, and lock the aptamer in a distinct open conformation. The competing oligo strand can be displaced by target-specific binding (Scheme 3). These processes require careful and meticulous design, re-engineering and expensive testing of various aptamer sequences to find the sensitive equilibrium between the non-binding state and the bound-state.48,101 Additionally, such aptamer engineering can often lead to degradation of the analytical characteristics of the aptamer. For example, Li et al. developed and utilized a competitive DNA strand strategy (scheme 3c) to develop a structure-switching aptamer for ATP detection. The affinity of the partially blocked aptamer is heavily affected, with a reported reduction in the equilibrium dissociation constant by up to 60 -fold, an effect of competition with the oligo strand..$^{83}$ Indeed, the length of the competing strand must be carefully designed otherwise the target will not be able to bind to the aptamer. This method is also not conveniently adaptable to $E-A B$ sensors since it requires addition of exogenous reagents and long incubation times to hybridize the immobilized probe to the competing oligonucleotide strand. Elsewhere, destabilization and addition of linking groups to separate binding sites have also 
been explored. White et al. engineered various derivatives of the original ATP aptamer to compare the effects of destabilization and pseudo-sandwich (bridged) designs (scheme $3, a$ and b) ${ }^{48}$ They concluded that destabilization provided the highest signal gain sensors, with ease of adaptability to E-AB sensing since the aptamer does not require any exogenous reagents for detection. White et al. also reported destabilization by truncation of the cocaine aptamer for efficient targetinduced sensing and similar results for the thrombin aptamer. ${ }^{87}$

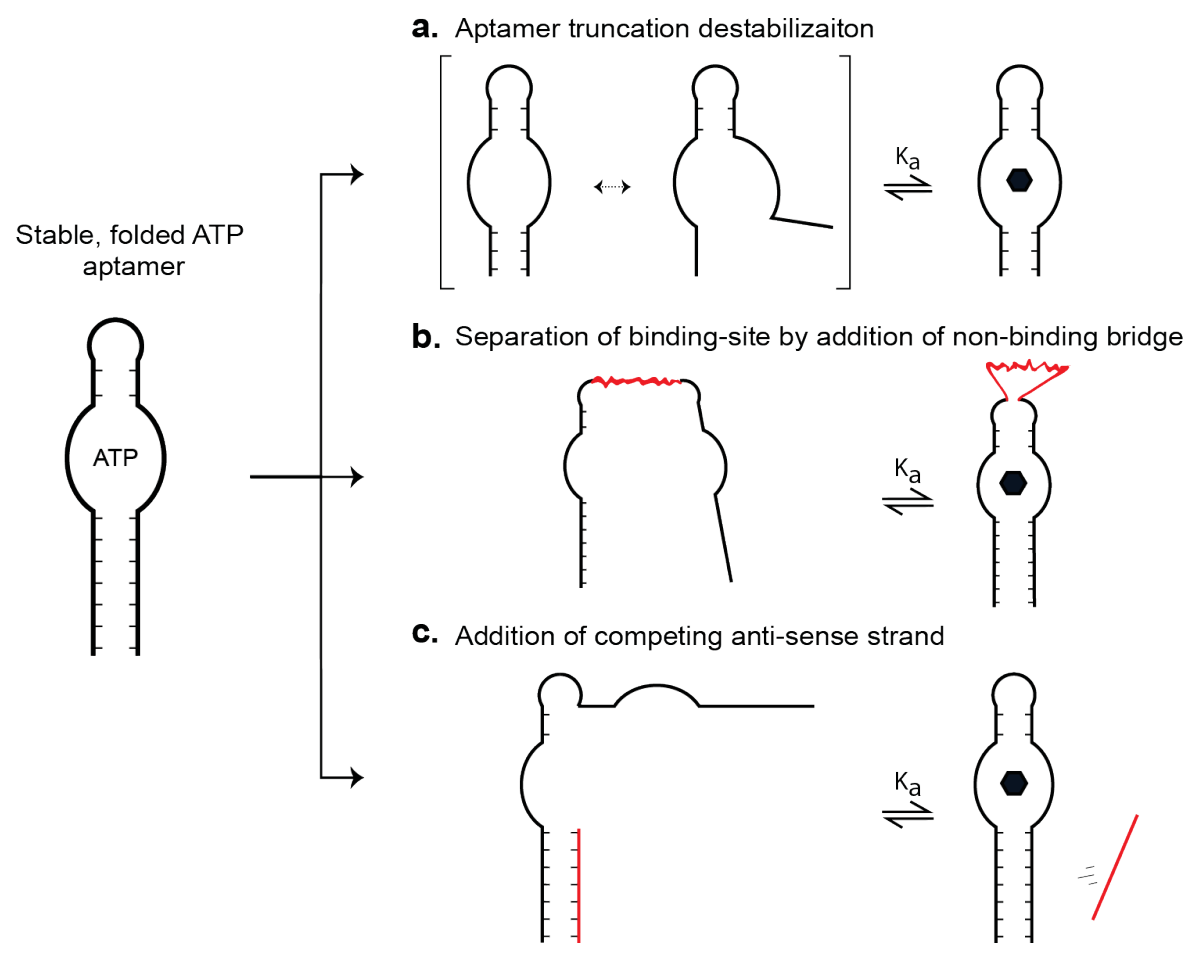

Scheme 3. Methods to add structure-switching function to aptamers.

Most reported aptamers display stable, mostly folded structures with long regions of rigid intramolecular helices (i.e., dsDNA). These aptamers adopt a semipermanent and optimal conformation to minimize energy barriers to bind the analyte. Several strategies have been developed to change the resting conformation including: (a) removing stabilizing base pairs from stem regions, causing unfolding of the aptamer structure that can only be repaired by target- 
aptamer binding. (b) Addition of long linker bridges that divide the binding motif into distinct sites that can only be reunited upon target addition. (c) Addition of a competing strand that blocks the binding site but can be displaced by targetinduced aptamer folding. ${ }^{48}$

Destabilization by truncation of various aptamers has resulted in aptamers that exhibit structure-switching functionality and have been successfully incorporated to $E-A B$ sensors. Unfortunately, design of such structures requires prior knowledge of the secondary structure of the aptamer and meticulous and expensive testing of multiple 'pilot' sequences. ${ }^{48,101}$ We describe a nuclease-based method that truncates the aptamer structure but is inhibited close to the binding site by formation of target-aptamer complexes, yielding aptamer sequences with structure-switching functionality without requiring complex, lengthy and expensive aptamer engineering.

Several purified enzymes exhibit exquisite specificity for hydrolysis of distinct DNA conformations (e.g., single-stranded vs. double-stranded DNA). For example, Lou et al. employed exonuclease I, which hydrolyzes single-stranded DNA but not double-stranded DNA, to create sensors for various aptamer targets including the small molecule cocaine. The employed aptamers exhibited disorganized and flexible structures prior to target binding that were susceptible to hydrolysis by the exonuclease I; Binding of target resulted in folding of the aptamer to a rigid doublestranded structure that was not susceptible to hydrolysis by exonuclease I. The undigested aptamer product could be quantified by addition of a DNA-binding fluorescent dye, with increasing fluorescence being proportional to target concentration in the sample. ${ }^{157}$ Unfortunately, the method requires prior 
knowledge of the folding dynamics of the aptamers and predicted structure changes upon target binding. Wang et al. recently described an exonuclease IIIbased system to instill structure-switching functionality to small molecule aptamers. Exonuclease III can digest double-stranded DNA specifically from blunt or recessed 3' ends in the 3' to 5' direction; Surprisingly, aptamer-target complexes inhibit digestion by exonuclease III 4-nt (nt: nucleotides) away from the presumed binding site. ${ }^{110}$ Enzyme truncation of several nucleotides yielded aptamers sequences with shorter stabilizing stems that were proven to undergo structureswitching functionality. Similarly, we developed a method utilizing T5 exonuclease as a catalyzing molecule to probe DNA aptamer conformation in the $5^{\prime}$ to $3^{\prime}$ direction to identify optimal structure-switching aptamer sequences. T5exonuclease has been previously shown to catalyze the hydrolysis of linear singlestranded DNA, double-stranded DNA, 5' overhangs and 3' overhangs in the 5' to 3' direction. ${ }^{111,112,114,219-221}$ Thus, T5 exonuclease is an excellent complement to exonuclease III, which acts in the 3' to 5' direction with similar enzymatic activity. I hypothesized that T5 exonuclease would be inhibited close to the presumed binding site by formation of aptamer-target complexes as its observed in the exonuclease III system. In the absence of target, the enzyme will freely track along the DNA sequence from the 5'- end and catalyze the hydrolysis of the aptamer molecule with activity for both the single- and double-stranded DNA aptamer conformations. In contrast, formation of an aptamer-target complex will inhibit the enzyme progress close to the binding site, providing truncation of the parent aptamer and yielding a minimal-length aptamer sequence which is preferentially 
unfolded in the absence of target, but can readily form a stable, enzyme-inhibiting folded structure upon target-binding. Herein, T5 is utilized to smartly truncate aptamers to yield structure-switching aptamers for small molecules including the synthetic cathinone MDPV and medical biomarkers DOG and ATP.

\subsection{Methods}

T5 Exonuclease was purchased from New England Biolabs (10 U/ $\mu \mathrm{L})$ and stored in $5 \mu \mathrm{L}$ aliquots at $-30^{\circ} \mathrm{C}$. Methylenedioxy pyrovalerone (MDPV) was purchased from Cayman Chemicals. Stock solutions were prepared in 100\% methanol at concentrations of $32 \mathrm{mM}$ and stored at $-30^{\circ} \mathrm{C}$. ATP was prepared in a buffered solution at a concentration of $37 \mathrm{mM}$, as measured using absorbance and an extinction coefficient of $15.4 \mathrm{mM}^{-1}$. DOG and DIS were prepared in $100 \%$ DMSO and stored at $-30^{\circ} \mathrm{C}$. Oligonucleotide sequences were purchased form IDT-DNA without purification with only standard desalting. DNA stock solutions were prepared by dissolving in nuclease-free water with average concentrations of 100 $\mu \mathrm{M}$. DNA concentration measurements were performed in a Nanodrop instrument with the specific extinction coefficient as provided by the company. Stocks were stored at $-30^{\circ} \mathrm{C}$ in $5 \mu \mathrm{L}$ aliquots. The following sequences were utilized: 
Table 4. DNA sequences, substitution and mutations.

\begin{tabular}{|c|c|}
\hline Name & Sequence $\left(5^{\prime}-3^{\prime}\right)$ \\
\hline MDPV.46 & CT TAC GAC TCA GGC ATT TTG CCG GGT AAC GAA GTT ACT GTC GTA AG \\
\hline MDPV.36 & CT TAC GAC TCA GGC ATT TTG CCG GGT AAC GAA GTT A \\
\hline MDPV.33 & CT TAC GAC TCA GGC ATT TTG CCG GGT AAC GAA G \\
\hline ATP.37 & AGA GAA CCT GGG GGA GTA TTA CGG AGG AAG GTA TAT T \\
\hline ATP. 25 & CGC A CCT GGG GGA GTA TTA CGG AGG \\
\hline DIS.37 & TCG GGA CGT GGA TTT TCC GCA TAC GAA GTT GTC CCG A \\
\hline DOG. & CTC GGG ACG TGG ATT TTC CAC AAA CCA GAA TGG TGT CCC GAG \\
\hline DOG.-3 & GGG ACG TGG ATT TTC CAC AAA CCA GAA TGG TGT CCC GAG \\
\hline DOG. +3 & TTT CTC GGG ACG TGG ATT TTC CAC AAA CCA GAA TGG TGT CCC GAG \\
\hline DOG. +7 & TTTT TTT CTC GGG ACG TGG ATT TTC CAC AAA CCA GAA TGG TGT CCC GAG \\
\hline DOG.m & TTT CTT ACG ACG TGG ATT TTC CAC AAA CCA GAA TGG TGT CGT AAG \\
\hline
\end{tabular}

Note: Red outline indicates the conserved aptamer binding sequence for DOG.

\subsubsection{T5 enzyme reaction}

DNA stocks were first diluted to $1 \mu \mathrm{M}$ and placed on a hot plate at $95^{\circ} \mathrm{C}$ for 10 minutes to completely denature the aptamer structure. The DNA solution was then placed in ice to obtain the lowest energy, folded sequence. The buffer employed for the enzymatic reaction was NEB-4 as suggested by the company for optimal catalysis (1x NEB-4: $20 \mathrm{mM}$ tris-acetate, $50 \mathrm{mM}$ potassium-acetate, $10 \mathrm{mM}$ magnesium acetate, $1 \mathrm{mM}$ dithiothreitol (DTT) at $\mathrm{pH}$ 7.9). The aptamer mixture was then incubated with the appropriate target for 30 minutes at $23^{\circ} \mathrm{C}$. MDPV stock solution was prepared by vacuum centrifuging to evaporate the methanol solution and isolate the solid MDPV. Water was added to the MDPV solid to appropriately match the desired concentration. T5 enzyme was diluted on ice to a final concentration of $1 \mathrm{U} / \mu \mathrm{L}$ and added to a $90 \%$ buffer mixture with DNA:enzyme ratio of $1 \mu \mathrm{M}: 0.1 \mathrm{U} / \mu \mathrm{L}$. The mixture was manually mixed and centrifuged prior to 
incubation at $23^{\circ} \mathrm{C}$. The reaction was stopped at various times by dilution with a stop mix gel loading buffer consisting of $3.5 \mathrm{M}$ urea and $50 \%$ formamide. (1 part DNA: 4 parts loading buffer)

\subsubsection{Gel Electrophoresis}

Denaturing gel electrophoresis ( $8 \mathrm{M}$ urea) was performed to separate and analyze the enzymatic reaction products. Polyacrylamide gels were prepared by mixing 5 $\mathrm{mL}$ DI, $10 \mathrm{~mL} 5 X$ TBE and $20 \mathrm{~mL}$ (40\% acrylamide) with $21 \mathrm{~g}$ of Urea. The solution was mixed on a hot plate at $30^{\circ} \mathrm{C}$ until the urea dissolved. Freshly prepared $10 \%$ APS and TEMED were then added and polymerization occurred at room temperature for 2 hours. The gels were pre-run at $450 \mathrm{~V}$ and the final sequence analysis was a two-step method, $100 \mathrm{~V}$ for 30 minutes and $400 \mathrm{~V}$ for 3 hours. Samples were loaded as prepared during the enzymatic reaction, $4 \mu \mathrm{L}$ per well. DNA markers were prepared in-house by mixing sequences with increasing lengths and matching the concentrations to obtain a uniform band intensity. Ladders were appropriately loaded to calculate the length of resulting products. Band intensity was compared to a control band without any enzyme to estimate the concentration of products.

\subsection{Results and discussion}

\subsubsection{MDPV aptamer truncation}

T5 exonuclease is utilized to probe the aptamer structure in the 5' to $3^{\prime}$ direction because of its ability to catalyze the hydrolysis of linear single-stranded and double-stranded DNA conformations. ${ }^{112}$ I hypothesized that T5 exonuclease would 
be inhibited close to the presumed binding site by formation of aptamer-target complexes. We utilized a novel methylenedioxy pyrovalerone (MDPV)-binding aptamer that was recently selected in our lab as a test bed. The enzymatic reaction was monitored by denaturing gel electrophoresis to identify the enzyme products and estimate their length by comparison to a DNA-marker. In the absence of MDPV, T5 can hydrolyze the aptamer structure yielding short oligonucleotide enzymatic product fragments of 21- and 22-nt (where -nt stands for nucleotides). Aptamer binding to the MDPV target severely inhibited T5 close to the presumed binding site, with a main enzyme reaction product of $41-n t$, which is 5 -nt less than the starting parent aptamer of 46-nt (Fig. 42a). Prior to T5-guided truncation, the aptamer stabilizing stem consists of 8-base pairs, most of which are presumably not involved in binding to the target but still stabilize the folded conformation and possibly prevent structure-switching upon target-binding (Fig. 42b). After T5guided truncation, the aptamer stem is reduced to 3-base pairs, which appears to be the minimum stem-length required for binding to the MDPV target. It is well established that reducing the stem length leads to increased possibility of structure-switching functionality, thus it is likely that the T5 truncation product can undergo structure-switching upon target-binding (fig. 42b, bottom). ${ }^{48,62,102}$ For quick comparison of the enzymatic reaction products, the aptamer structure is color-coded. In the absence of target, the enzyme reaction product is represented by a red outline. In the presence of target, the enzyme reaction product is represented as a combination of the red and a blue outline. 

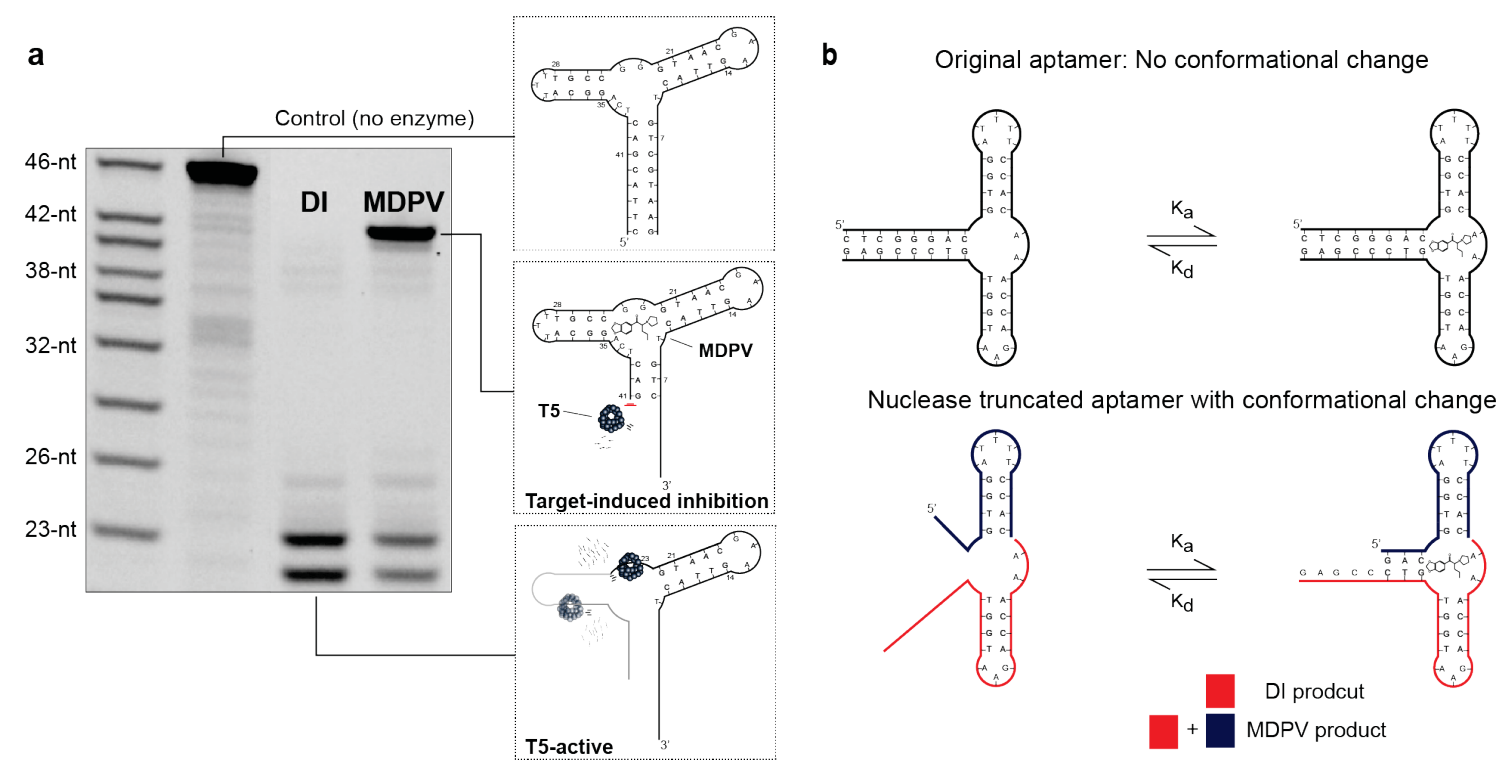

Nuclease truncated aptamer with conformational change
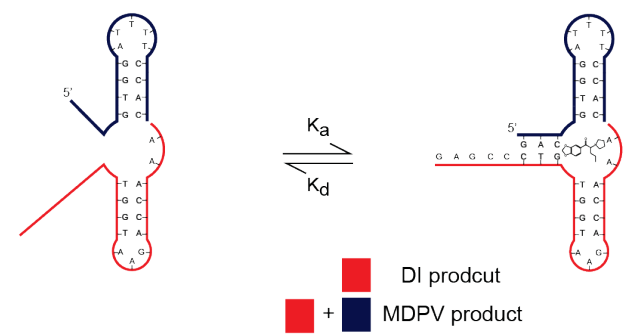

Figure 42. T5-guided truncation of an MDPV-binding aptamer yielding a structureswitching sequence.

(a) T5 exonuclease was utilized to digest the MDPV-aptamer. In the absence of target, T5 catalyzes the hydrolysis of the aptamer structure, yielding short fragments of 22- and 21-nt. Formation of an MDPV-aptamer complex inhibits T5 activity 3-nt away from the presumed binding site, producing a distinct product of 41-nt. A control band is shown without addition of enzyme and a DNA marker is utilized to approximate the length of the enzymatic products. (b) The originally selected aptamer displays a long stabilizing stem which prevents target-induced structure-switching (top). The T5-truncated aptamer has a much shorter stabilizing stem of 3 base-pairs which possibly exhibits target-induced structure-switching (bottom). The colored lines indicate the enzyme reaction products in the absence (red; 19- and 20-nt) and presence (blue + red; 41-nt) of MDPV. [T5] $=0.1 \mathrm{U} / \mu \mathrm{L}$, $[\mathrm{DNA}]=1 \mu \mathrm{M},[\mathrm{MDPV}]=320 \mu \mathrm{M}$ at $23^{\circ} \mathrm{C}$ for 120 minutes .

The T5 reaction was stopped at different times by mixing with formamide and EDTA to examine the reaction products over time. A control sample of constant concentration was utilized to estimate the concentration of the enzymatic products. In the absence of a binding target, several enzymatic products are observed over a time window of five hours. In the absence of target, T5 reaction for 15 minutes yields a singular 41 -nt product with an estimated concentration of $800 \mathrm{nM}(80 \%$ of 
the starting material). Interestingly, the 41-nt product is still present even after two hours of reaction, suggesting the enzyme unbinds with the DNA at this point possibly because of a change in the conformation of the structure and must rebind to digest the new aptamer conformation. After $120 \mathrm{~min}$, the 41-nt product is only faintly present with a concentration of $100 \mathrm{nM}$ and it is eventually completely digested after 5 hours (fig. 43). In contrast, two reaction products are observed after 15 minutes of incubation with T5 in the presence of MDPV with lengths of 42 nt and 41-nt and concentrations of $450 \mathrm{nM}$ each (fig. 43, plotted as a combined concentration). After 120 minutes, most of the 42 -nt product is enzymatically converted to the 41-nt product and the estimated concentration of the 41-nt product at this point was $600 \mathrm{nM}$ (fig. 43). Even after 5 hours of T5 digestion, the MDPVaptamer mixture exhibits a 41-nt product with an estimated concentration of 550 nM (fig. 43). Thus, we proved that formation of MVPD-aptamer complex inhibits T5 activity and the inhibition product of 41-nt possibly has different conformations in the absence and presence of MDPV. This method can possibly identify a structure-switching aptamer in a single experiment, without requirements for complex aptamer engineering or labor-intensive testing of multiple sequences. 


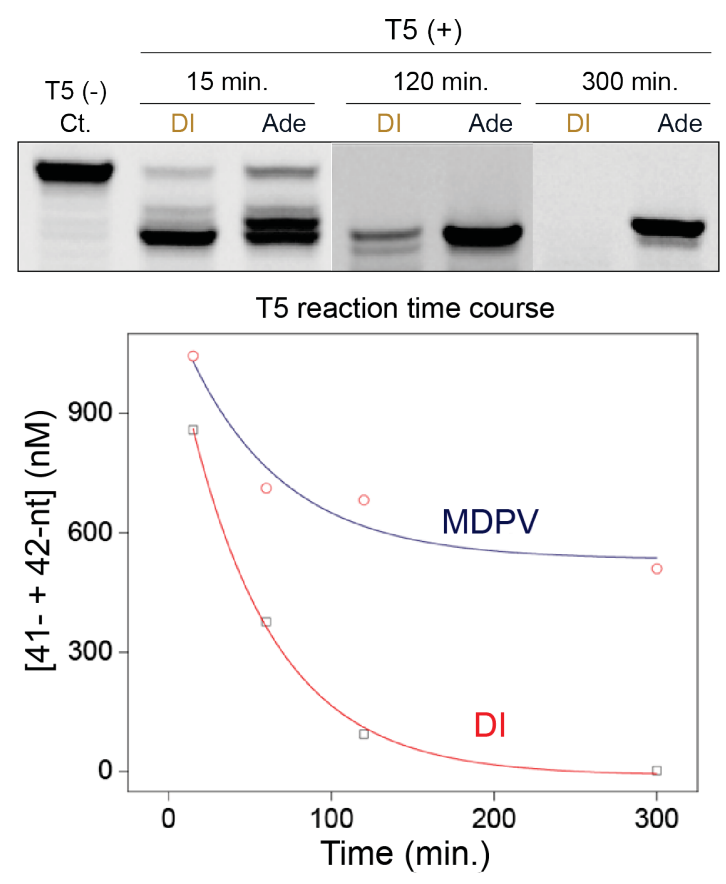

Figure 43. Time course of $\mathrm{T} 5$ reaction and characterization of enzyme reaction products visualized utilizing gel electrophoresis.

T5-exonuclease can quickly digest nucleotides that are not involved in binding to the target and within two hours most of the parent aptamer is hydrolyzed in the absence of target. However, in the presence of MDPV, formation of the aptamertarget complex inhibits T5 digestion and results in a truncated product of 41-nt. This suggests that formation of a folded aptamer-target complex inhibits the enzyme progress. The intensity of the bands is converted to concentration by comparing to the control band which has a known concentration. $[\mathrm{T} 5]=0.1 \mathrm{U} / \mu \mathrm{L}$, $[D N A]=1 \mu \mathrm{M},[\mathrm{MDPV}]=320 \mu \mathrm{M}$ at $23^{\circ} \mathrm{C}$.

To confirm that T5 inhibition is a result of formation of MDPV-aptamer complexes and not a result of direct inhibition by MDPV, we designed truncated sequences of the MDPV aptamer which do not form the presumed hydrophobic binding site. The MDPV aptamer structure was manually truncated by deletion of 10- and 13-nt from the 3' end yielding aptamer variants of 36-nt and 33-nt. The predicted folding was calculated with Mfold, a webserver to predict nucleic acid folding, to predict whether the three-way junction could be formed in the 33- and 36-nt aptamer 
variants. ${ }^{222}$ These shorter fragments are not predicted to form a three-way junction because of the absence of complementary base-pairs, inhibiting target binding (see Mfold predicted structures in figure 44). T5 truncation of the non-binding sequences resulted in reaction products that were identical whether in the presence or absence of MDPV. For example, the 36-nt aptamer exhibited a main product of $28-\mathrm{nt}$ and a concentration of $175 \mathrm{nM}$, about $17 \%$ of the starting material in the presence or absence of MDPV (fig. 44). The 33-nt aptamer after T5 truncation yielding a major product of 25-nt regardless of the absence or presence of MDPV. T5 is only inhibited when the 46-nt binding aptamer is utilized, with a 41-nt inhibition product of $>500 \mathrm{nM}$ (fig. 44). Thus, the inhibition of T5 is directly caused by binding of MDPV to the aptamer. To easily visualize and compare the products of the enzymatic reaction to the starting structure, a color-guide outline is utilized. For example, the starting aptamer of $46-\mathrm{nt}$ is a combination of the gray, red and blue outlines. T5-exonuclease removes 5-nt from the starting structure yielding a main product of 41-nt in the presence of MDPV, represented by the blue and red outlines combined. In the absence of target, the main products are shown in a red outline, with lengths of 22- and 21-nt (Fig. 44, bottom). From this scheme, it can be observed that only the 46-nt aptamer bound to MDPV inhibits T5 activity and yields a different product in the presence of MDPV. 

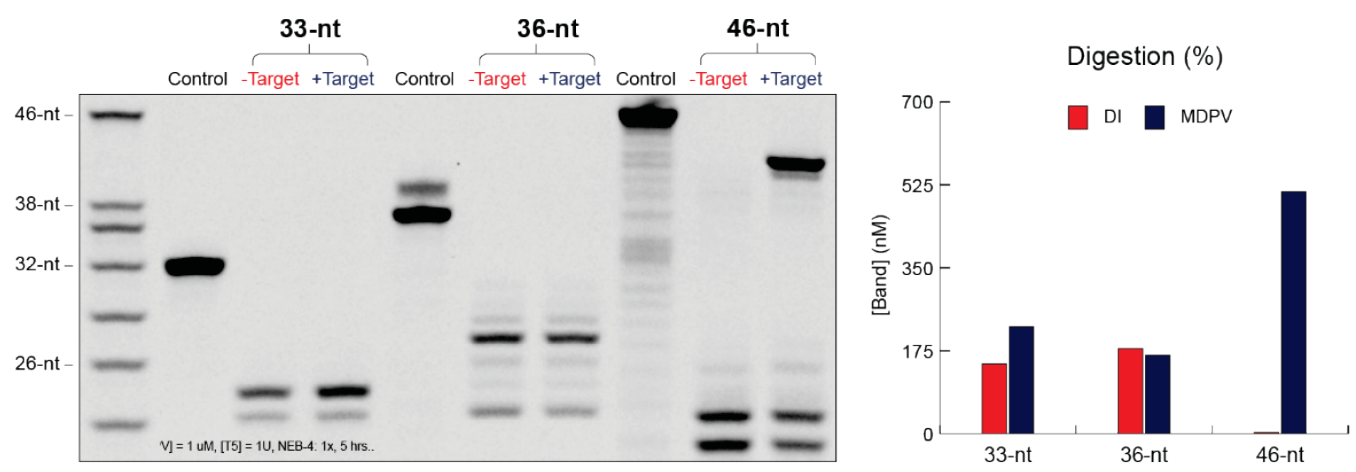

33-nt

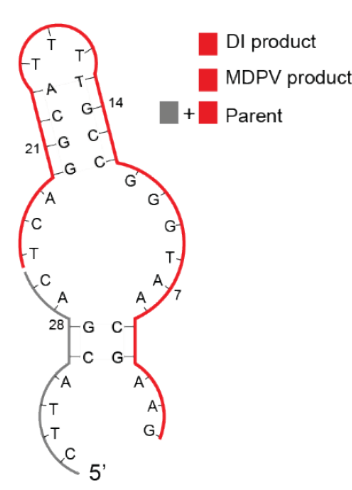

36-nt

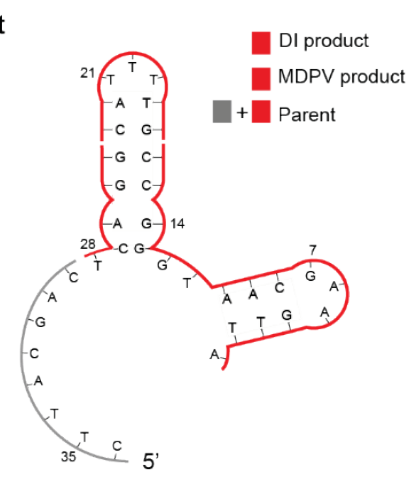

46-nt

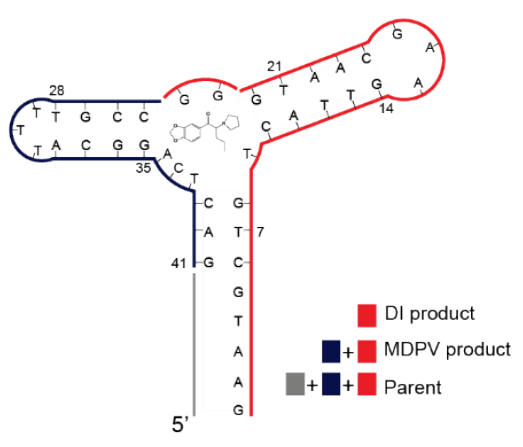

Figure 44. T5 inhibition is caused by formation of a MDPV-aptamer complex and not by MDPV itself.

(a) Gel electrophoresis enzyme products of different aptamer sequences in the presence and absence of target. The truncated non-binding sequences (33-nt and 36-nt) do not exhibit any MDPV-induced inhibition. However, the 46-nt binding aptamer exhibits significant MDPV-induced inhibition of T5 digestion with a singular product of 41-nt and concentration of $>500 \mathrm{nM}$ (represented by the blue and red outline combined). In the absence of target, short products of 22- and 21$\mathrm{nt}$ are observed (represented by the red outline). [T5] $=0.1 \mathrm{U} / \mu \mathrm{L}$, [DNA] $=1 \mu \mathrm{M}$, $[\mathrm{MDPV}]=320 \mu \mathrm{M}$ at $23^{\circ} \mathrm{C}$ for 120 minutes.

\subsubsection{Comparison with T7-exonuclease}

Enzymatic inhibition by formation of aptamer-target complexes has been demonstrated with exonuclease III (3'-5' direction) previously and with T5exonuclease (5'-3' direction) in this work. To study whether this is phenomenon is generalizable, I employed T7-exonuclease to determine if aptamer-target complexes could also inhibit T7 activity. T7-exonucelase has been widely reported 
to have incredible specificity for double-stranded DNA, with activity from blunt or recessed $5^{\prime}$ ends. ${ }^{223-225}$ Indeed, a singular mismatched base-pair can result in inhibition of $\mathrm{T} 7$ enzymatic activity. ${ }^{226}$ I hypothesized that $\mathrm{T} 7$ would be able to hydrolyze aptamer structures with stable stems but be inhibited close to the binding site as observed with T5 and exonuclease III. We tested the same 46-nt MDPVbinding aptamer to compare $\mathrm{T} 5$ and $\mathrm{T} 7$ for aptamer truncation. $\mathrm{T} 5$ can truncate the aptamer to yield a product of $41-n t$ in the presence of MDPV but T7 is not able to digest the aptamer structure in the absence or presence of target (fig. 45). Even after 4 hours of $\mathrm{T} 7$ reaction, the same parent aptamer is observed suggesting the enzyme is not able to catalyze hydrolysis of this aptamer structure even though it exhibits a favorable double-stranded substrate. It is notable that T7 exonuclease does not have activity for single-stranded DNA. In contrast, both T5-exonucelase and exonuclease III have both single- and double-stranded activity. At least in this case and under the current experimental conditions, it appears that only T5 exonuclease is capable of aptamer truncation. 

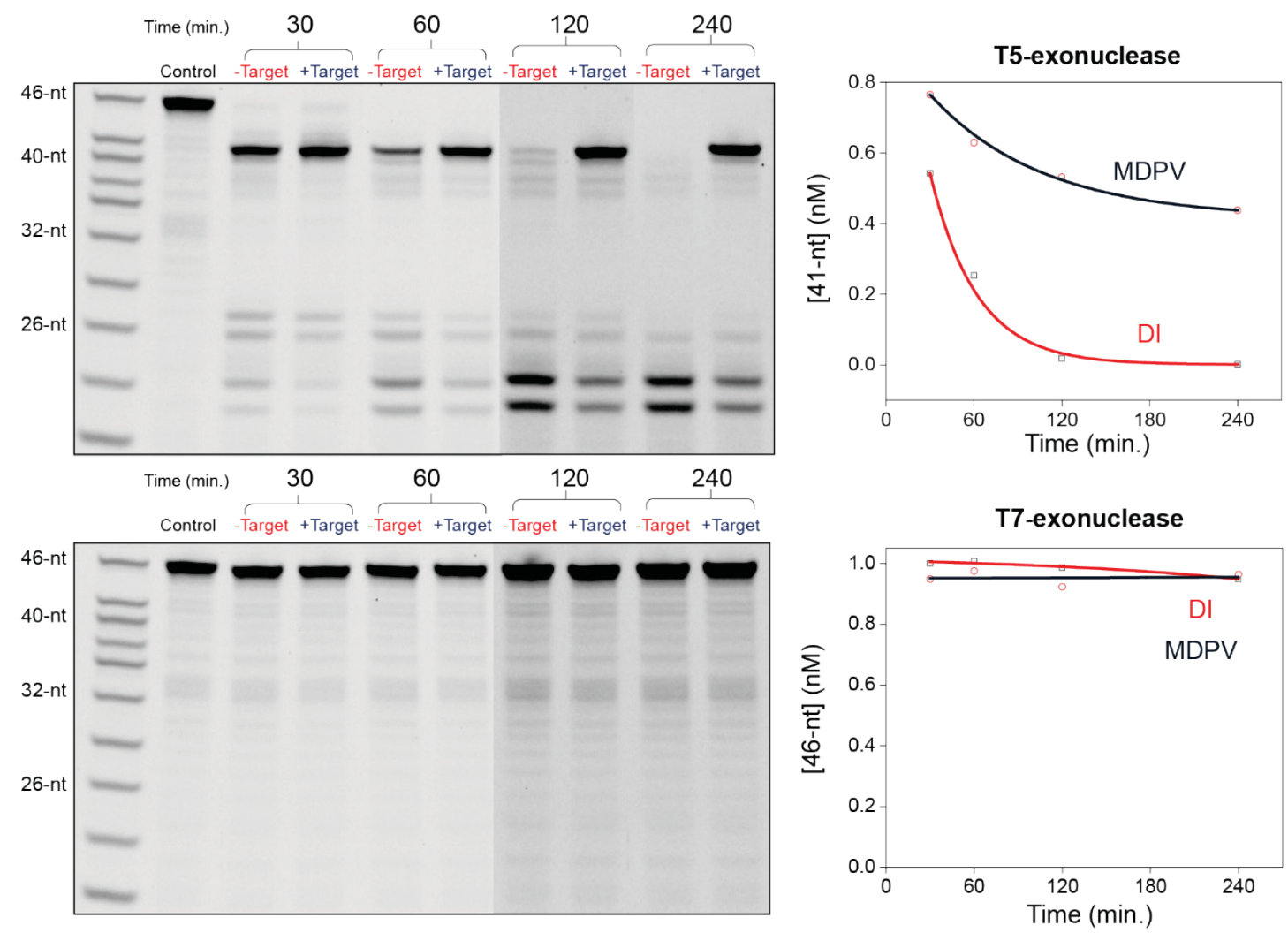

Figure 45. Comparison of aptamer truncation with T5- and T7-exonuclease.

Two enzyme-based methods were tested with the MDPV binding aptamer. As expected, T5 could differentiate between aptamer-bound and aptamer-free structures. However, T7 did not digest either structure even though it has been previously reported to be highly specific for double-stranded DNA. [T5] $=0.1 \mathrm{U} / \mu \mathrm{L}$, $[\mathrm{T7}]=0.2 \mathrm{U} / \mu \mathrm{L},[\mathrm{DNA}]=1 \mu \mathrm{M},[\mathrm{MDPV}]=320 \mu \mathrm{M}$ at $23^{\circ} \mathrm{C}$.

\subsubsection{ATP-binding aptamer truncation}

I studied T5 truncation of the ATP-binding aptamer to prove T5 could truncate aptamer structures other than three-way junctions. The ATP aptamer has a stemloop structure with a 4-base pair stabilizing stem and a reportedly folded conformation even in the absence of target, with limited structural change upon target binding. ${ }^{208,210,227,228}$ Indeed, White et al. performed urea denaturation studies and found that $>99.6 \%$ of the ATP-aptamer molecules are folded in the absence of target. ${ }^{48}$ In contrast, reducing the stem length by 2 base-pairs results 
in the unfolded aptamer confirmation dominating the conformational equilibrium. I hypothesized that T5 would be inhibited as it approached the presumed binding site by formation of the ATP-aptamer complex. I modified the previously reported ATP-binding aptamer by adding 3' and 5' overhangs of 5-nt each but otherwise conserved the same sequence as the original ATP aptamer of Huizenga et al. ${ }^{47}$ The overhangs have been reported to provide a better binding substrate for T5. ${ }^{113}$ Thus, the utilized ATP-binding aptamer has a 37-nt long sequence (fig. 46, 37-nt). As predicted, T5 can truncate 6-nt from the starting aptamer sequence of 37-nt, yielding a product of 31 -nt corresponding to a structure with a 3 base-pair stem (fig. 46). The concentration of the 31-nt product was plotted over the time course of the reaction and exhibited a consistent value of $>900 \mathrm{nM}$ even after 5 hours (90\% starting concentration). I previously utilized a similar construct with stem length of 3 base-pairs for detection of adenosine and proved that it underwent significant target-induced conformational changes (see chapter 4). Thus, T5 truncation of the ATP aptamer can yield an aptamer sequence that possibly undergoes structure-switching. In the absence of target, two products are observed over the reaction time course of five hours with lengths of 31-nt and 30nt and corresponding stems of 3 and 2 base-pairs, respectively (fig. 46). After 5 hours only the $30-n t$ product remains at a concentration of nearly $300 \mathrm{nM}$. The $30-$ nt structure with the 2 base-pair stem was previously proven to be unfolded in the conformational equilibrium ${ }^{48}$, thus it appears from these results that under the current conditions, digestion of single-stranded DNA by T5 is slow. After 8 hours, the 30-nt band is consumed in the absence of target but still retained in the 
presence of target (data not shown).

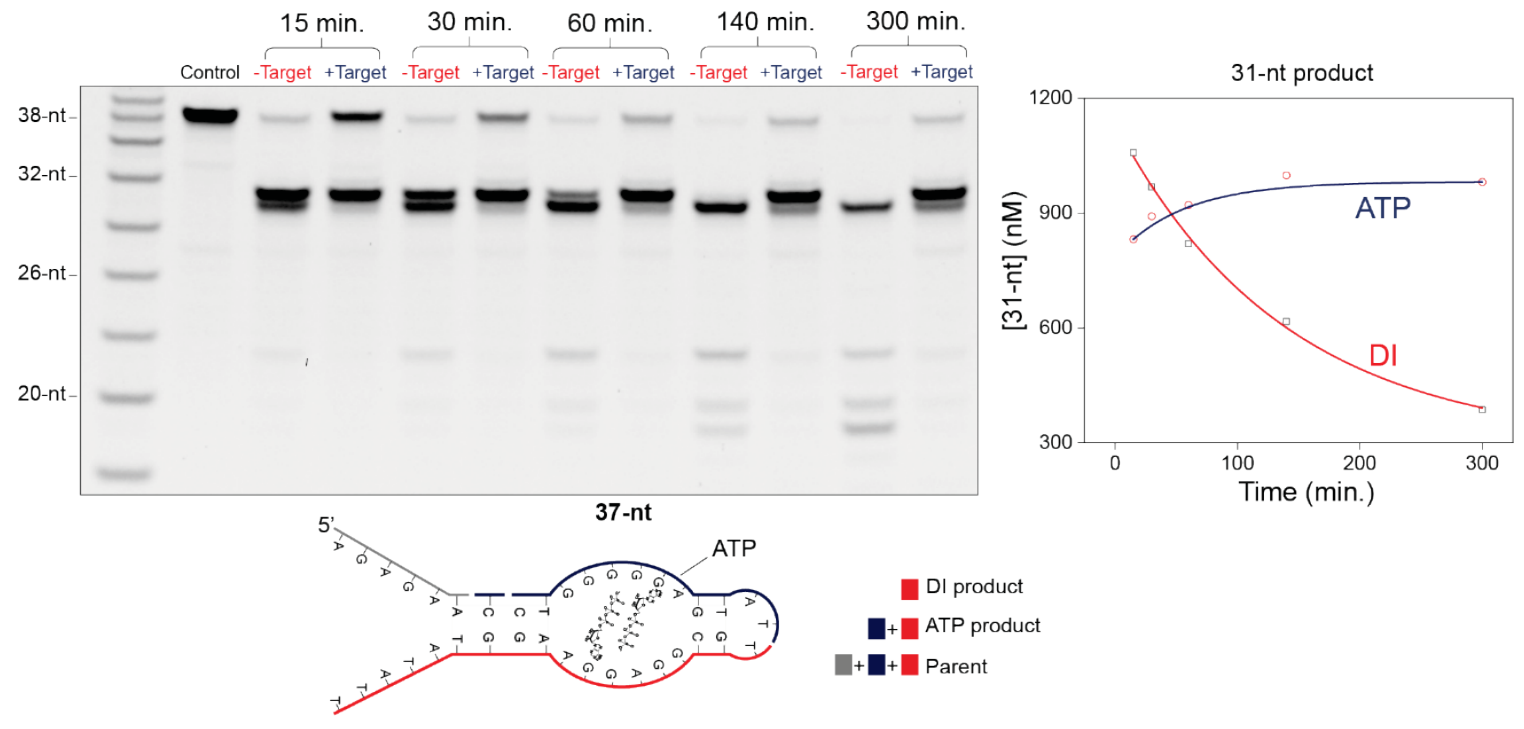

Figure 46. Time course of T5 reaction with ATP-binding aptamer.

Digestion of the aptamer proceeds quickly at first as the 5' overhang is a good enzyme substrate, with most of the parent aptamer being processed within $15 \mathrm{~min}$. In the absence of target, the enzyme can digest most of the structure, yielding a minor product of 30-nt and concentration of $300 \mathrm{nM}$. However, in the presence of target, T5 truncation yields two products of 31- and 30-nt. The major product is 31nt with a corresponding stem of 3 base-pairs and a consistent concentration of $>900 \mathrm{nM}$ over the five-hour reaction window. (right) The band intensity is converted to concentration by utilizing a control band of known concentration without enzyme addition. $[\mathrm{T} 5]=0.1 \mathrm{U} / \mu \mathrm{L},[\mathrm{DNA}]=1 \mu \mathrm{M},[\mathrm{ATP}]=500 \mu \mathrm{M}$ at $23^{\circ} \mathrm{C}$.

An ATP non-binding sequence was tested to confirm that ATP did not inhibit T5 directly. The non-binding sequence was designed by removal of 8-nt from the 3' end of the aptamer sequence and 2-nt from the 5' end and termed ATP.25. The 25-nt sequence is not able to form the binding pocket and it also lacks an essential binding nucleotide as has been previously described. ${ }^{47} \mathrm{~T} 5$ truncation of the ATP. 25 yielded the same 20-nt product regardless of the presence or absence of ATP, proving that ATP does not actively inhibit the enzyme (fig. 47). In contrast, the 37- 
nt ATP binding aptamer inhibited T5 by formation of the ATP-aptamer complex, yielding the described 31-nt product. A secondary product is observed if the reaction is left to proceed for longer time, with truncation of 7 -nt yielding a product of 30 -nt which correspondingly has a stem of 2 base-pairs. This 30 -nt structure has also been previously reported to retain binding affinity for ATP, thus it appears T5 can recognize two separate aptamer products that can undergo structureswitching upon target binding ${ }^{48}$ The same color-guide is employed to describe the different reaction products in the presence and absence of ATP as described in the last section. The T5-guided strategy enables simpler and faster identification of the structure-switching sequences for stem-loop structures.

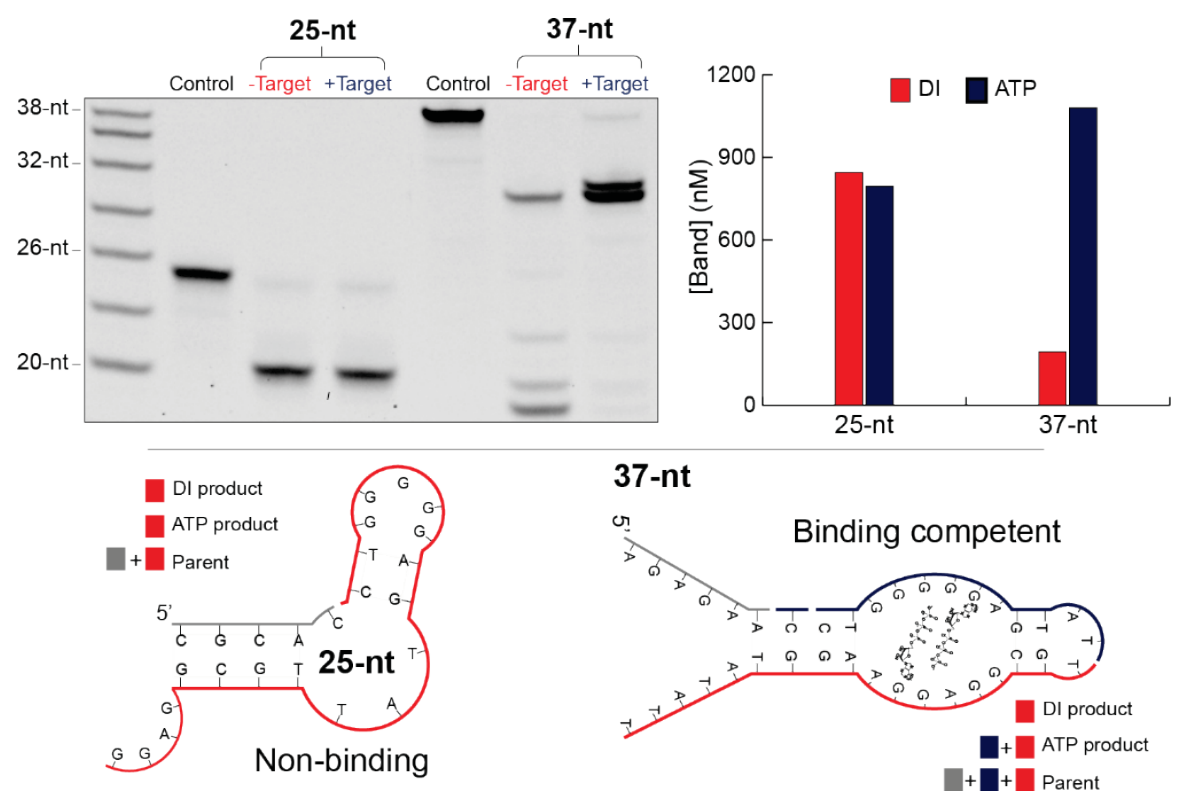

Figure 47. T5 inhibition is caused by formation of aptamer-ATP complexes and not by ATP directly.

A manually truncated non-binding sequence was tested (25-nt) and yielded the same product of 20-nt regardless of ATP presence or absence. This proves that ATP does not directly inhibit T5 activity. In contrast, the 37-nt ATP-binding sequence underwent T5-guided truncation yielding products of 31- and 30-nt. Both 
of these products have been previously shown to retain affinity for ATP. The column plot represents the band intensity as a measurement of concentration. [T5] $=0.1 \mathrm{U} / \mu \mathrm{L},[\mathrm{DNA}]=1 \mu \mathrm{M},[\mathrm{ATP}]=500 \mu \mathrm{M}$ at $23^{\circ} \mathrm{C}$ for 340 minutes .

\subsubsection{DIS-binding aptamer truncation}

To further test the generality of the method, I tested aptamers which specifically bind the small molecule steroids deoxycorticosterone 21-glucoside (DOG) and dehydroisoandrosterone 3-sulfate (DIS). Stojanovic et al. previously isolated these three-way junction aptamers, which display long stabilizing stems and a binding pocket at the junction of the stems. ${ }^{229}$ The formation of the junction is required for binding as it creates a hydrophobic environment by display of aromatic groups of unstacked nucleotides at the junction, favoring binding of hydrophobic molecules. ${ }^{58} \mathrm{I}$ utilized T5 to initially truncate the DIS-binding aptamer to yield the minimum number of base-pairs required for binding of DIS while maximizing the structure-switching ability of the aptamer. The DIS-binding aptamer structure was almost fully digested in the absence of binding target with nearly zero bands present in the lane of this sample (fig. 48, DIS). Incubation with DIS resulted in nearly complete inhibition of the enzyme with concentrations of the product band being nearly uniform from the starting concentration, and the major product being the parent aptamer of 37-nt and a minor product of 36-nt (fig. 48, DIS). Truncation was not observed with the DIS-binding aptamer, suggesting that all the nucleotides in the stem are necessary for a high-affinity binding interaction with DIS. This is a possibility as formation of the binding pocket is required for binding of hydrophobic

molecules. ${ }^{58,229}$ Indeed, a recently reported and improved DIS-binding aptamer by Stojanovic et al. has equally long stabilizing stems, suggesting the necessity for all 
nucleotides for high-affinity binding. ${ }^{230}$ Thus, it is possible that truncation of these steroid-binding aptamers is not possible or necessary.
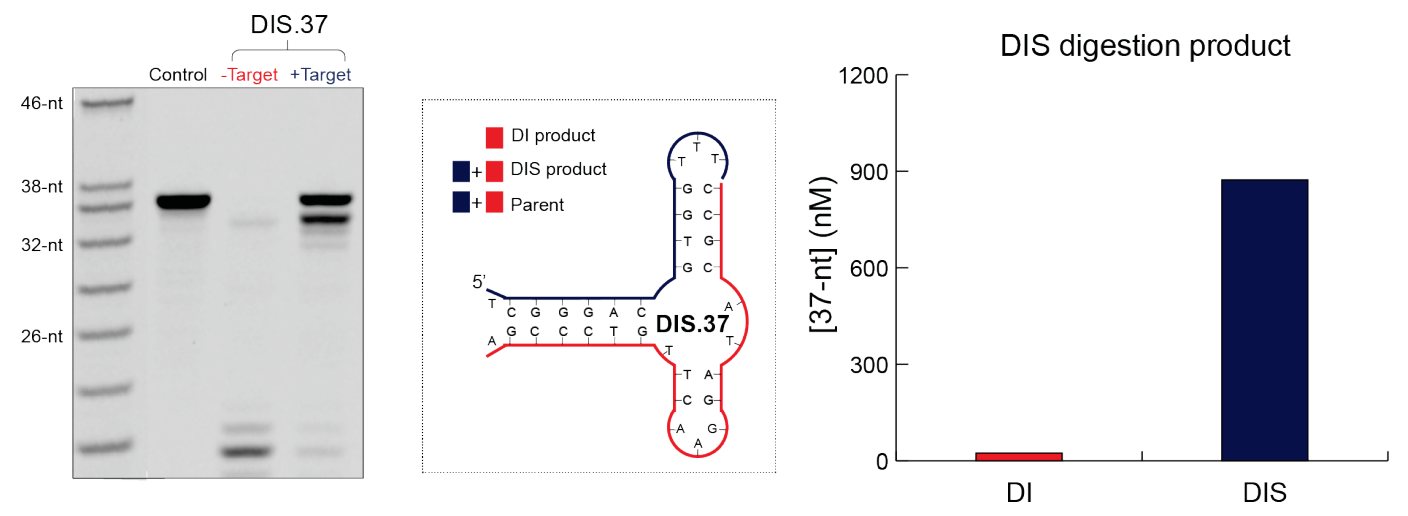

Figure 48. T5-guided truncation of DIS-binding aptamer to identify structureswitching fragments.

The DIS aptamer was digested in the absence of target as expected, with only a faint product band of 23-nt being observed in the gel image. Addition of DIS resulted in nearly complete inhibition of T5 yielding products that did not differ in length when compared to the control sample without enzyme. This might be possibly because all the nucleotides in the stem are required to stabilize a highaffinity interaction with DIS. [T5] $=0.1 \mathrm{U} / \mu \mathrm{L},[\mathrm{DNA}]=1 \mu \mathrm{M}$, [Steroid $]=500 \mu \mathrm{M}$ at $23^{\circ} \mathrm{C}$ for 135 minutes.

Direct inhibition of T5 by the steroid DIS was tested by incubating DIS with a control DNA sequence (an ATP-binding aptamer) that does not bind DIS. The T5 enzyme digested the control sequence at equal rates in the presence or absence of DIS, with multiple product bands present and concentration of the parent band below $300 \mathrm{nM}$ after 2 hours, demonstrating that DIS does not inhibit the enzyme directly (fig. 49a). The enzyme T7 exonuclease was also tested and did not digest the control sequence in any case (fig. 49a). It is mentioned and included because an increase in the intensity of the bands in the presence of DIS was present in all samples and was attributed to overlap between the fluorescence intensity of SYBR 
gold (the fluorescence DNA stain) and DIS (fig. 49c). ${ }^{231}$ Thus, clearly the enzyme is not inhibited directly by DIS but rather it is possible that all the nucleotides in the stem are necessary for target binding under the employed conditions.

a

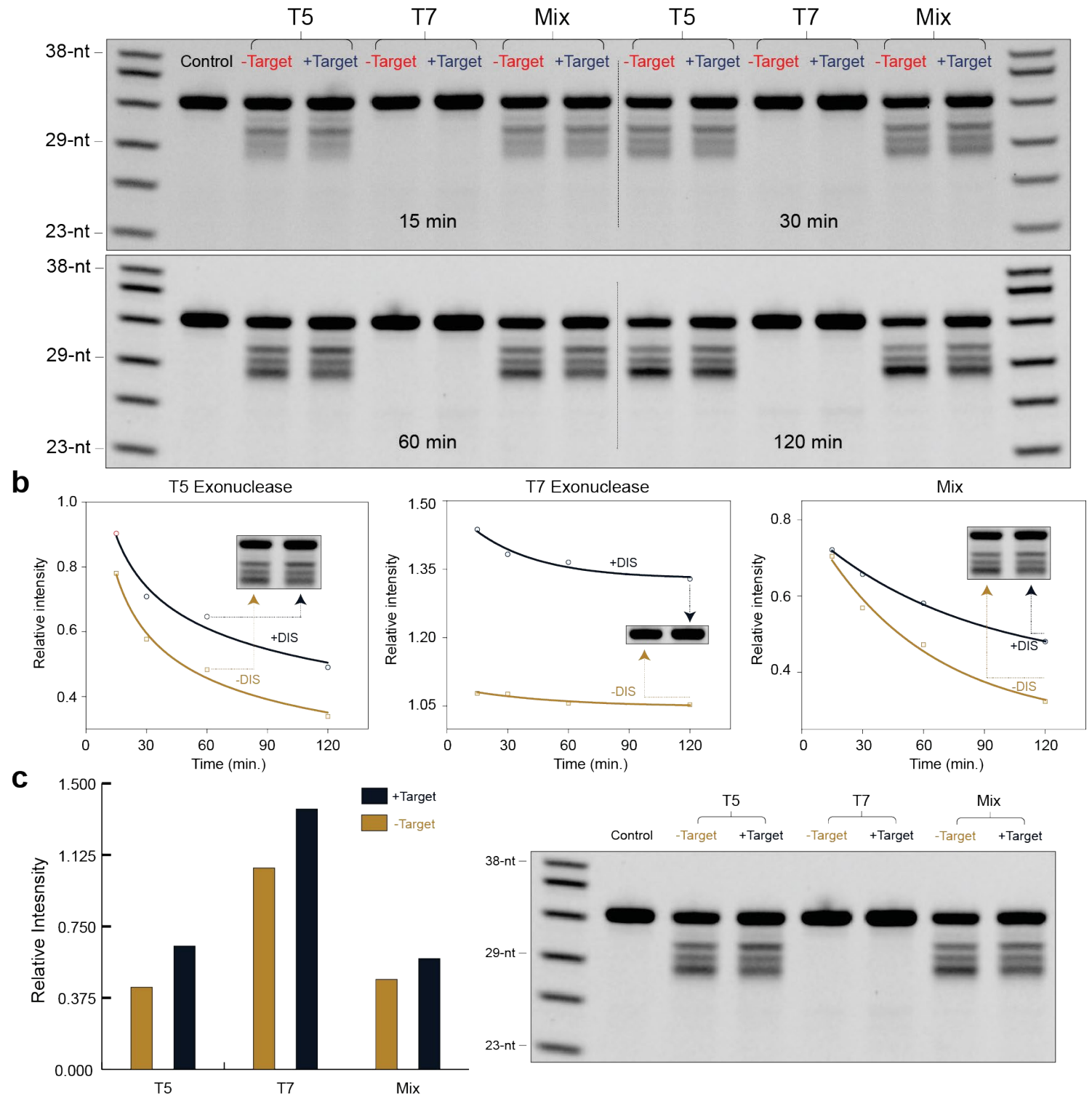

Figure 49. Test of T5 inhibition by the steroid molecule DIS.

(a) Gel electrophoresis of reaction products of an ATP-binding aptamer incubated with DI or DIS at varying times. T5 enzyme digestion of the control DNA was slow 
and it did not vary in the presence or absence of DIS. T7 exonuclease also did not exhibit digestion of the control aptamer and a mixture of T5 and T7 exhibited similar response as T5 alone. (b) Time course plots of the band intensity (converted to concentration) exhibit decreasing band intensity over time, because of enzymatic hydrolysis and formation of shorter fragments. (c) A difference in the intensity was observed in the presence of DIS that was attributed to fluorescence overlap between DIS and the DNA staining dye, SYBR gold. The difference in intensity was present with both enzymes. This test proves that DIS does not inhibit T5 directly.

\subsubsection{T5 activity on DOG-binding aptamer}

The DOG aptamer demonstrated the slowest rate of hydrolysis of all the aptamers tested (data not shown). I hypothesized that addition of a 5'- overhang would aid in the rate of digestion of the DOG aptamer, as the T5 enzyme can bind 5' overhangs with higher affinity when compared to blunt double-stranded DNA. ${ }^{232}$ We modified the DOG aptamer with 5'- overhangs of varying lengths, and a 5' recessed end to study their effect on enzyme rate. Interestingly, the digestion of the 5'- overhang was very fast ( $<5 \mathrm{~min}$.) but it appeared the enzyme would unbind from the aptamer structure upon cleaving the 5 ' overhang, as a major product was observed at 41-nt regardless of the length of the 5' overhang. A 5' overhang of +7 nt resulted in a truncation of 8-nt, yielding a 41-nt product and truncation of the +3nt overhang resulted in truncation of 4-nt, yielding the same 41-nt product (fig. 50, 5 mins.). Digestion of the blunted or 5' recessed aptamer structures was much slower, necessitating more than five hours to digest in the absence of target (fig. 49,300 mins). In the absence of target, a faint product is observed at $37-\mathrm{nt}$, corresponding to a short 3 base-pair stem which likely signals the stem position where structure-switching occurs. In the presence of DOG, various products are 
observed although the majority only exhibit minor truncation. Like the DIS aptamer, the DOG aptamer likely requires all nucleotides in the stem for high-affinity interaction with the target.

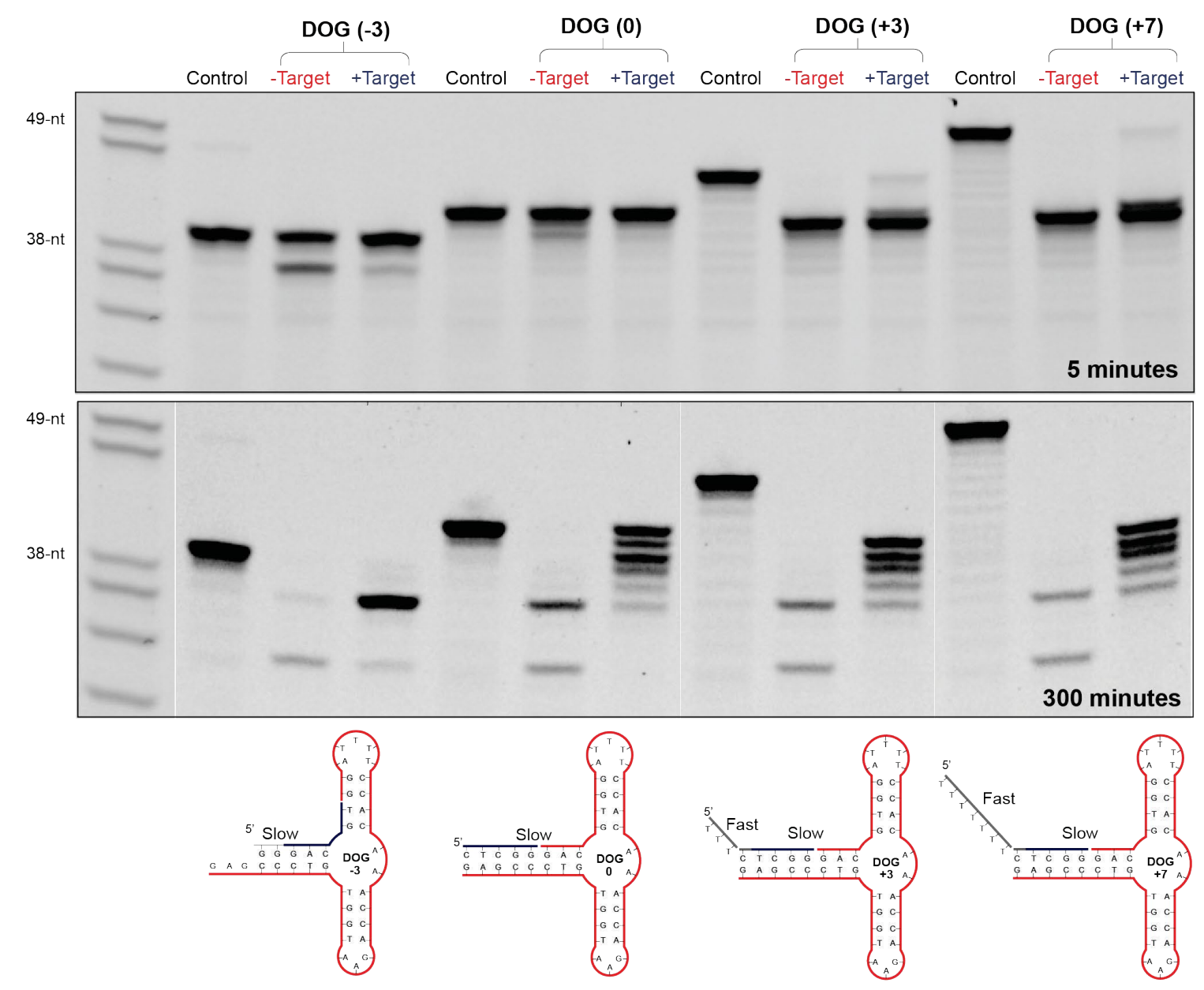

Figure 50. Digestion rate of T5 on 5' overhangs and double-stranded DNA.

Various aptamers were synthesized with increasingly longer 5 ' overhangs to study the role of binding of the enzyme to 5' overhangs and 5' blunt ends. The 5' overhangs exhibited fast digestion rates, with the extensions of all lengths being quickly cleaved at the double-stranded junction. Destabilization of the stem by reducing the number of base pairs resulted in identification of a truncated aptamer that displayed target-induced inhibition of the enzyme. This sequence presumably is the minimum number of base pairs that is required for dsDNA formation and inhibition of T5. $[\mathrm{T5}]=0.1 \mathrm{U} / \mu \mathrm{L},[\mathrm{DNA}]=1 \mu \mathrm{M}$, [Steroid] $=500 \mu \mathrm{M}$ at $23^{\circ} \mathrm{C}$. 
Additionally, we tested a sequence where the DOG aptamer had a 5' recessed end by 3-nt, thus only having a stem of 5 base-pairs. These 5' recessed structures also reportedly promote binding of T5 compared to blunt DNA. ${ }^{232}$ Indeed, T5 can catalyze the hydrolysis of this structure in the absence of target and addition of target inhibits the digestion, yielding a truncated sequence with a 3-base pair stem (fig. 50, DOG.-3). Mfold simulations predict that the 5-base pair aptamer can form a stable, folded structure that presumably does not undergo significant structureswitching upon target-biding. However, the T5-selected 3 base-pair aptamer is predicted to form a highly flexible, unfolded sequence which can possibly undergo target-induced structure-switching. ${ }^{222}$

A time-course of the reaction between $\mathrm{T} 5$ and the $5^{\prime}$ recessed DOG aptamer variant is shown in figure 51. In the absence of target, T5 can digest the aptamer structure until it reaches a product of $37-n t$ yielding a 3 base-pair stem. It is possible that at this point, the enzyme disengages from the aptamer structure because of a conformational change in the DNA. After 5 hours, the product at $37-$ nt is completely consumed by the enzyme (fig. 51). In the presence of DOG, T5 is inhibited close to the presumed binding site with a major product of $37-n$ t being observed even after 5 hours of reaction with a concentration of $>600 \mathrm{nM}$ remaining (fig. 51). The recessed aptamer can be utilized in combination with T5 to yield a structureswitching DOG aptamer. 


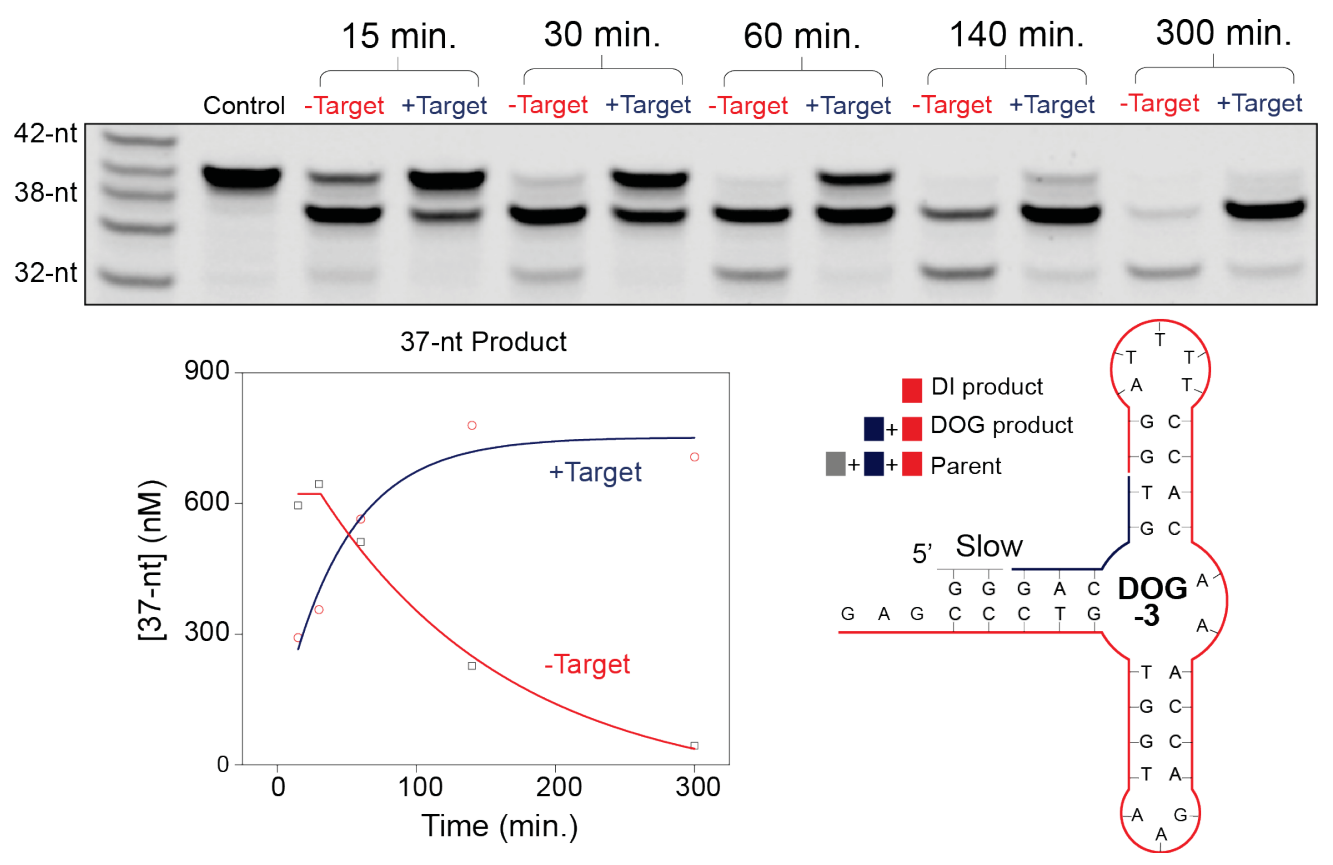

Figure 51. Time course of T5 digestion of DOG.-3 aptamer structure and enzymatic products visualized using gel electrophoresis.

The enzyme reaction was stopped by mixing of reaction buffer with stop-mix loading buffer containing high concentrations of EDTA, formamide and urea. The aptamer is digested at a faster rate in the absence of target, but the enzyme is significantly inhibited upon addition of DOG because of formation of DOG-aptamer complex. After 5 hours, the enzyme truncates 2-nt to yield an aptamer of 37-nt and a corresponding stem of 3 base-pairs (blue line) which appears to be the minimum number of base-pairs required for binding of target and addition of structureswitching functionality. $[\mathrm{T5}]=0.1 \mathrm{U} / \mu \mathrm{L},[\mathrm{DNA}]=1 \mu \mathrm{M}$, [Steroid $]=500 \mu \mathrm{M}$ at $23^{\circ} \mathrm{C}$.

\subsubsection{Engineering a DOG-binding aptamer for T5 truncation}

A DOG-binding aptamer was engineered by changing the stem sequence to closely match the initial 5 base pairs of the MDPV stem, which does not contain more than 2 consecutive C-G base pairs and is shown to be readily digested by T5 (red nucleotides in figure 52). The redesigned sequence can be fully digested in the absence of target at a much faster rate than the original DOG aptamer (Fig. 52). Increased digestion was also observed in the presence of DOG, with a 
truncated aptamer sequence being identified by the nuclease as a 3-base pair stem, matching the result using the shorter 5-base pair aptamer (Fig. 52). A smaller concentration of the $37-\mathrm{nt}$ binding product is observed $>300 \mathrm{nM}$. This might be a result of incomplete digestion of larger fragments as seen in the gel but could also be an effect of reduced affinity for DOG because of the nucleotide substitutions in the stem. It appears that truncation of steroid binding aptamers, which necessitate highly stable stems, might not be practical or necessary.
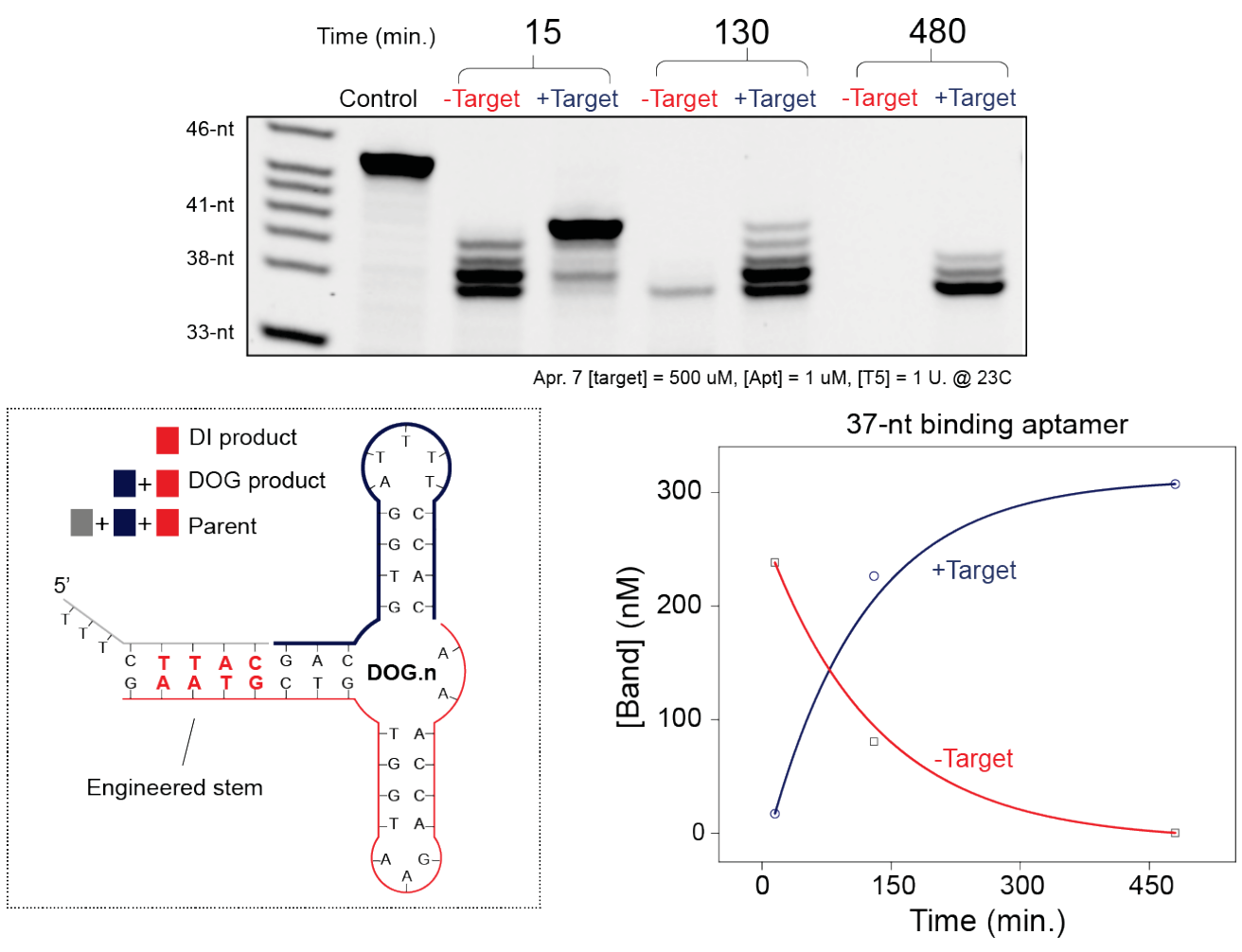

Figure 52. T5-guided truncation of redesigned DOG aptamer sequence.

(a) Time course of digestion of redesigned sequence with a 5' overhang and several mutated nucleotides that match the MDPV stem. Digestion is faster as compared to the original sequence and in the absence of target completely consumes the parent aptamer within 130 minutes. In the presence of target, the main product has a 3 base-pair stem stabilized by target-binding which inhibits further enzymatic digestion. The band concentration is plotted as a function of time. 
The blue line in the illustrations represents the product sequence that is identified in the presence of target and the red line in the absence of target. [T5] $=0.1 \mathrm{U} / \mu \mathrm{L}$, $[\mathrm{DNA}]=1 \mu \mathrm{M}$, [Steroid $]=500 \mu \mathrm{M}$ at $23^{\circ} \mathrm{C}$.

\subsection{Conclusion}

A T5-guided truncation method is demonstrated to easily and quickly truncate aptamers to yield aptamer sequences which possibly display structure-switching functionality and maintain high target affinity. We hypothesized that T5 exonuclease would be inhibited by formation of aptamer-target complexes as is seen in small molecule footprinting studies utilizing exonuclease III and DNAse I. ${ }^{110,233}$ Indeed, formation of aptamer-target complexes inhibit the enzyme activity, with single or dual products only being observed in the presence of the target as compared to empty lanes in the absence of target. The method is used to identify aptamer fragments with structure-switching functionality for targets including MDPV, DOG and ATP. The enzyme T5 exonuclease demonstrates a unique ability to recognize the essential aptamer sequence for binding while removing any unessential nucleotides. The enzyme T7 exonuclease is unable to perform such function. This strategy will be a key tool -fast, simple, widely available, and low cost- in development of E-AB sensors which require aptamers with structureswitching functionality. 


\section{CHAPTER 6}

\section{Summary and future directions}

\subsection{Summary}

The dissertation describes methods to engineer aptamers to increase analytical performance, modulate specificity and add functionality with the goal of overcoming practical limitations of current aptamer-based sensors, especially for small-molecule detection in real-world sample matrices. In chapter 3 , it was discovered that ATMND could bind to the cocaine aptamer, modulating its fluorescence when bound (quenched) or free (fluorescent) in solution. Addition of cocaine would displace the ATMND molecule from the aptamer binding pocket, resulting in an intense fluorescence signal that could be correlated to cocaine concentration. Additionally, a cocaine aptamer was engineered with 10 -fold higher affinity for both molecules as compared to previously reported aptamers. The method was applied to detect cocaine in biological samples such as urine, saliva and serum. The sensitivity of the method was over 50 -fold higher than other reported aptamer-based methods. The improvement in sensitivity was correlated to higher affinity for cocaine and sensitive fluorescence signaling from ATMND displacement.

In chapter 4 , an adenosine-specific aptamer was engineered by rational mutation of the aptamer sequence. A method to quickly screen the mutated sequences was also introduced, which aided in the identification of the adenosine-specific aptamer. The aptamer was truncated to introduce structure-switching functionality and adapted to an electrochemical transducer. The sensor could detect low 
concentrations of adenosine in undiluted serum and exhibited minimal response to ATP, ADP and AMP. The sensitivity of the sensor was improved by 40 -fold when compared to previously reported aptamer-sensors for undiluted serum measurements of adenosine.

In chapter 5, a nuclease-guided method was developed to intelligently truncate aptamer sequences yielding aptamer fragments with structure-switching functionality. As opposed to trial-and-error methods, no prior knowledge of the binding site is required to yield truncated aptamers. Aptamers with structureswitching functionality can be used directly in a variety of methods that can be directly applied in complex samples. For example, electrochemical aptamer-based sensors can be directly utilized in undiluted blood because of the extreme selectivity of electrochemical detection. The method will allow for development of E-AB sensors for new aptamers in a simple, fast and low-cost manner.

\subsection{Future}

Aptamer-based cocaine detection with our fluorescence method suffered from auto-fluorescence in urine and serum. When the matrix by itself was excited at 358 $\mathrm{nm}$, endogenous molecules in the matrix gave high fluorescence at the monitored emission wavelength of $405 \mathrm{~nm}$. Thus, detection in complex matrices was limited to diluted samples which resulted in limited sensitivity. Additionally, the cocaine aptamer that was engineered displayed strong cross-reactivity with several small molecules that could be present in biological fluids. To solve both issues, Yu et al. develop a split-aptamer assay incorporating a fluorophore that is out of the range 
of most of the endogenous fluorescent molecules. ${ }^{234}$ Thus, this improved method can detect cocaine in $10 \%$ saliva below the recommended cut-off limit established by the European Union's Driving under the Influence of Drugs, Alcohol and Medicines program. A similar approach could be taken to identify a fluorophore outside of the interference range that can bind in the aptamer pocket and be displaced by the drug target. For example, Sato et al. reported an ATMND-thiazole orange conjugate that exhibited the same affinity for abasic sites as demonstrated by ATMND alone but with significantly higher excitation $(506 \mathrm{~nm})$ and emission (530 nm) wavelengths, which are well outside of the auto-fluorescence window of biological fluids. ${ }^{235}$ Indeed, it was demonstrated that the conjugate exhibited nanomolar affinity for cytosine and thymine opposite the abasic site but did not bind strongly with purine nucleotides. Thus, it is likely that the conjugate could retain the same affinity for the cocaine aptamer pocket and be utilized as a signal reporting dye.

We demonstrate the first specific sensor for detection of adenosine in undiluted serum over similar molecules such as ATP, ADP and AMP. I would like to apply this sensor technology to measure real patient samples. The test would include a control healthy population and a diseased population. Generally, adenosine concentration is much higher in diseased states $(>3 \mu \mathrm{M})$ vs. healthy individuals $(<1$ $\mu \mathrm{M}){ }^{73,75,77}$ The sensor could be used to calculate the concentration of both populations and determine a cut-off concentration for disease diagnosis. The sensor could also be used to profile the activity of adenosine regulating enzymes to discover new small molecule inhibitors of these enzymes which are often over 
or under-expressed in diseased states. An important limitation of current measurement techniques for adenosine is the inability to measure adenosine close to the cell surface, where it is often produced and accumulated. Aptamers have been previously immobilized onto cell membranes by conjugation with an intercalating group. For example, Tokunaga et al. previously conjugated the ATPcross reactive aptamer with tocopherol as a direct method to anchor the aptamer onto cell membranes and monitor ATP release by a fluorescence reporter directly modified in the aptamer structure. ${ }^{184} \mathrm{~A}$ similar approach could be utilized to measure adenosine in situ, providing much more accurate representation of real adenosine concentration and production dynamics. To increase the sensitivity, we can also modify the aptamer to only bind a single adenosine molecule per aptamer instead of the current two. Zhang et al. recently determined that the classic ATP aptamer could be engineered to bind only one molecule per aptamer while keeping the same stem-loop structure, a similar approach could be taken with our new adenosine-specific aptamer. ${ }^{197}$ This would immediately increase the sensitivity by at least two-fold.

Aptamer truncation was also demonstrated with $\mathrm{T} 5$ exonuclease as a key tool to develop structure-switching aptamers which have been widely used in electrochemical-based sensors. However, the structure-switching functionality of the aptamers was not directly measured. Thus, experiments such as circular dichroism could be performed to measure the changes in the truncated aptamer structure before and after target binding and further comparing the results to fully folded aptamers. Urea denaturation studies could also be attempted to observe 
whether the truncated sequences are fully denatured in the absence of target. Additionally, isothermal titration calorimetry must be employed to determine the binding affinity and thermodynamics of binding of the truncated sequences as compared to the original parent aptamer. Finally, the identified structure-switching aptamers must be functionalized with an electroactive group and utilized in E-AB sensors to determine their analytical characteristics and functionality in complex samples.

The T5 exonuclease method can also be used independent of aptamer-sensors to yield truncated aptamer sequences that can provide higher specificity than fully folded aptamers. We previously demonstrated that the truncated aptamer for cocaine does not bind ATMND favorably. Several non-specific binders do not actively induce structure-switching; thus, specificity is improved upon truncation. The method could also potentially be used for development of a SELEX process as the enzyme can differentiate between target-binding and non-binding sequences, as T5 will fully digest the non-binding sequences while leaving targetbinding aptamers behind. 


\section{References}

1. $\mathrm{Wu}, \mathrm{A}$. H. B. A selected history and future of immunoassay development and applications in clinical chemistry. Clin. Chim. Acta 369, 119-124 (2006).

2. Marx, V. Finding the right antibody for the job. Nat. Methods. 10, 703-707 (2013).

3. Groff, K., Brown, J. \& Clippinger, A. J. Modern affinity reagents:

Recombinant antibodies and aptamers. Biotechnol. Adv. 33, 1787-1798 (2015).

4. Qu, H. et al. Rapid and Label-Free Strategy to Isolate Aptamers for Metal lons. ACS. Nano. 10, 7558-7565 (2016).

5. Baker, M. Blame it on the antibodies. Nature. 521, 274-276 (2015).

6. Prassas, I. \& Diamandis, E. P. Translational researchers beware! Unreliable commercial immunoassays (ELISAs) can jeopardize your research. Clin. Chem. Lab. Med. 52, 765-766 (2014).

7. Armbruster, D. A., Schwarzhoff, R. H., Hubster, E. C. \& Liserio, M. K. Enzyme immunoassay, kinetic microparticle immunoassay, radioimmunoassay, and fluorescence polarization immunoassay compared for drugs-of-abuse screening. Clin. Chem. 39, 2137-2146 (1993).

8. Cho, E. J., Lee, J.-W. \& Ellington, A. D. Applications of Aptamers as Sensors. Annu. Rev. Anal. Chem. 2, 241-264 (2009).

9. Wang, J. et al. Particle display: a quantitative screening method for generating high-affinity aptamers. Angew. Chem. Int. Ed. Engl. 53, 4796801 (2014).

10. Arroyo-Currás, N. et al. Real-time measurement of small molecules directly in awake, ambulatory animals. Proc. Natl. Acad. Sci. 114, 645-650 (2017).

11. Liu, J. \& Lu, Y. Fast colorimetric sensing of adenosine and cocaine based on a general sensor design involving aptamers and nanoparticles. Angew. Chem. Int. Ed. 45, 90-94 (2006).

12. Liu, J. \& Lu, Y. Preparation of aptamer-linked gold nanoparticle purple aggregates for colorimetric sensing of analytes. Nat. Protoc. 1, 246-52 (2006).

13. Trachman, R. J., Truong, L. \& Ferré-D'Amaré, A. R. Structural Principles of Fluorescent RNA Aptamers. Trends Pharmacol. Sci. 38, 928-939(2017). 
14. E. Wang, R., Zhang, Y., Cai, J., Cai, W. \& Gao, T. Aptamer-Based Fluorescent Biosensors. Curr. Med. Chem. 18, 4175-4184(2011).

15. Eissa, S. \& Zourob, M. Aptamer- Based Label-Free Electrochemical Biosensor Array for the Detection of Total and Glycated Hemoglobin in Human Whole Blood. Sci. Rep. 7, 1-8 (2017).

16. Lu, Y. et al. Aptamer-based electrochemical sensors with aptamercomplementary DNA oligonucleotides as probe. Anal. Chem. 80, 18831890 (2008).

17. Pfeiffer, F. \& Mayer, G. Selection and Biosensor Application of Aptamers for Small Molecules. Front. Chem. 4, 1-21 (2016).

18. Bhalla, N., Jolly, P., Formisano, N. \& Estrela, P. Introduction to biosensors. Essays Biochem. 60, 1-8 (2016).

19. Mehrotra, P. Biosensors and their applications - A review. J. Oral Biol. Craniofacial Res. 6, 153-159 (2016).

20. Turner, A. P. F. et al. Biosensors: sense and sensibility. Chem. Soc. Rev. 42, 3184 (2013).

21. Chard, T. Review: Pregnancy tests: A review. Hum. Reprod. 7,701-710 (1992).

22. Gnoth, C. \& Johnson, S. Strips of Hope: Accuracy of Home Pregnancy Tests and New Developments. Geburtshilfe Frauenheilkd. 74, 661-669 (2014).

23. Berger, P. \& Sturgeon, C. Pregnancy testing with hCG - future prospects. Trends Endocrinol. Metab. 25, 637-648 (2014).

24. Yeh, C.-H., Zhao, Z.-Q., Shen, P.-L. \& Lin, Y.-C. Optimization of an Optical Inspection System Based on the Taguchi Method for Quantitative Analysis of Point-of-Care Testing. Sensors 14, 16148-16158 (2014).

25. Koczula, K. M. \& Gallotta, A. Lateral flow assays. Essays Biochem. 60, $111-120$ (2016).

26. Ruigrok, V. J., Levisson, M., Eppink, M. H., Smidt, H. \& van der Oost, J. Alternative affinity tools: More attractive than antibodies? Biochem. J. 436, 1-13 (2011).

27. Zhou, J. \& Rossi, J. Aptamers as targeted therapeutics: Current potential and challenges. Nat. Rev. Drug Discov. 16, 181-202 (2017). 
28. Kobayashi, N. \& Got, J. Advances in clinical chemistry. 36, (2001).

29. Darwish, I. A. Immunoassay Methods and their Applications in Pharmaceutical Analysis: Basic Methodology and Recent Advances. Int. J. Biomed. Sci. 2, 217-35 (2006).

30. Powers, J. L. et al. A direct, competitive enzyme-linked immunosorbent assay (ELISA) as a quantitative technique for small molecules. J. Chem. Educ. 89, 1587-1590 (2012).

31. O'Kennedy, R., Byrne, M., O'Fagain, C. \& Berns, G. A Review of EnzymeImmunoassay and a Description of a Competitive Enzyme-Linked Immunosorbent Assay for the Detection of Immunoglobulin Concentrations. Biochem. Educ. 18, 136-140 (1990).

32. Lu, N. T. \& Taylor, B. G. Drug screening and confirmation by GC-MS: Comparison of EMIT II and Online KIMS against 10 drugs between US and England laboratories. Forensic. Sci. Int. 157, 106-116 (2006).

33. Beckman-Coulter. Cocaine Metabolite Assay. (2010).

34. Sanavio, B. \& Krol, S. On the Slow Diffusion of Point-of-Care Systems in Therapeutic Drug Monitoring. Front. Bioeng. Biotechnol. 3, 1-15(2015).

35. Zhou, W., Jimmy Huang, P.-J., Ding, J. \& Liu, J. Aptamer-based biosensors for biomedical diagnostics. Analyst 139, 2627 (2014).

36. Schoukroun-Barnes, L. R. et al. Reagentless, Structure-Switching, Electrochemical Aptamer-Based Sensors. Annu. Rev. Anal. Chem. 9, 163181 (2016).

37. Yang, D. et al. Aptamer-based biosensors for detection of lead( <scp>ii</scp> ) ion: a review. Anal. Methods 9, 1976-1990 (2017).

38. Ellington, A. D. \& Szostak, J. W. In vitro selection of RNA molecules that bind specific ligands. Nature 346, 818 (1990).

39. Tuerk, C. \& Gold, L. Systematic Evolution of Ligands by Exponential Enrichment: RNA ligands to bacteriophage T4 DNA polymearase. Science 249, 505-510 (1990).

40. Gold, L., Janjic, N., Jarvis, T., Schneider, D. \& Walker, J. J. Aptamers and the RNA World, Past and Present Aptamers and the RNA World, Past and Present. Cold Spring Hard Perspect Biol 4, a003582 (2012).

41. Gold, L. SELEX: How It Happened and Where It will Go. J. Mol. Evol. 81, 140-143 (2015). 
42. Stoltenburg, R., Reinemann, C. \& Strehlitz, B. SELEX--a (r)evolutionary method to generate high-affinity nucleic acid ligands. Biomol. Eng. 24, 381-403 (2007).

43. Famulok, M. \& Mayer, G. Aptamers and SELEX in Chemistry \& Biology. Chem. Biol. 21, 1055-1058 (2014).

44. Darmostuk, M., Rimpelova, S., Gbelcova, H. \& Ruml, T. Current approaches in SELEX: An update to aptamer selection technology. Biotechnol. Adv. 33, 1141-1161 (2014).

45. Yang, K. A., Pei, R. \& Stojanovic, M. N. In vitro selection and amplification protocols for isolation of aptameric sensors for small molecules. Methods 106, 58-65 (2016).

46. Li, L. Le, Ge, P., Selvin, P. R. \& Lu, Y. Direct detection of adenosine in undiluted serum using a luminescent aptamer sensor attached to a terbium complex. Anal. Chem. 84, 7852-7856 (2012).

47. Huizenga, D. E. \& Szostak, J. W. A DNA Aptamer That Binds Adenosine and ATP. Biochemistry 34, 656-665 (1995).

48. White, R. J., Rowe, A. A. \& Plaxco, K. W. Re-engineering aptamers to support reagentless, self-reporting electrochemical sensors. Analyst 135, 589 (2010).

49. Gabay, M. The Federal Controlled Substances Act: Schedules and Pharmacy Registration. Hosp. Pharm. 48, 473-474 (2013).

50. Kirkpatrick2, R. M. M. 1 and M. B. Clinical Use of cocaine. Drug Saf. 9, 433-458 (1993).

51. UNODC. World Drug Report 2017. (2017).

52. Riezzo, I. et al. Side effects of cocaine abuse: Multiorgan toxicity and pathological consequences. Curr. Med. Chem. 19, 5624-5646(2012).

53. Robinson, J. E., Agoglia, A. E., Fish, E. W., Krouse, M. C. \& Malanga, C. J. Mephedrone (4-methylmethcathinone) and intracranial self-stimulation in C57BL/6J mice: comparison to cocaine. Behav Brain Res 234, 76-81 (2012).

54. Fox, T. P., Oliver, G. \& Ellis, S. M. The Destructive Capacity of Drug Abuse : An Overview Exploring the Harmful Potential of Drug Abuse Both to the Individual and to Society. Hindawi Publ. Corp. 2013, (2013). 
55. Gonz, M. L. Determination of cocaine and its metabolites in human urine by gas chromatography / mass spectrometry after simultaneous use of cocaine and ethanol. 13, (1995).

56. Schramm, W., Craig, P. a, Smith, R. H. \& Berger, G. E. Cocaine and benzoylecgonine in saliva, serum, and urine. Clin. Chem. 39, 481-7 (1993).

57. Stojanovic, M. N., Prada, P. \& Landry, D. W. Fluorescent Sensors Based on Aptamer Self-assembly. J. Am. Chem. Soc. 122, 11547-11548 (2000).

58. Stojanovic, et al. Cross reactive arrays based on three way junctions. J. Am. Chem. Soc. 125, 6085 (2003).

59. Stojanovic, M. N., Prada, P. De \& Landry, D. W. Aptamer-Based Folding Fluorescent Sensor for Cocaine. J. Am. Chem. Soc. 4, 4928-4931(2001).

60. Stojanovic, M. N. \& Landry, D. W. Aptamer-Based colorimetric probe for cocaine. J. Am. Chem. Soc. 124, 9678-9679 (2002).

61. Kent, A. D., Spiropulos, N. G. \& Heemstra, J. M. General approach for engineering small-molecule-binding DNA split aptamers. Anal. Chem. 85, 9916-23 (2013).

62. Neves, M. A., Reinstein, O. \& Johnson, P. E. Defining a stem lengthdependent binding mechanism for the cocaine-binding aptamer. A combined NMR and calorimetry study. Biochemistry 49, 8478-8487 (2010).

63. Kang, K., Sachan, A., Nilsen-Hamilton, M. \& Shrotriya, P. Aptamer functionalized microcantilever sensors for cocaine detection. Langmuir 27, 14696-702 (2011).

64. Swensen, J. S. et al. Continuous, real-time monitoring of cocaine in undiluted blood serum via a microfluidic, electrochemical aptamer-based sensor. J. Am. Chem. Soc. 131, 4262-4266 (2009).

65. Sato, Y. et al. Influence of substituent modifications on the binding of 2amino-1,8-naphthyridines to cytosine opposite an AP site in DNA duplexes: thermodynamic characterization. Nucleic Acids Res. 37, 1411-22 (2009).

66. Sato, Y. et al. Influence of substituent modifications on the binding of 2amino-1,8-naphthyridines to cytosine opposite an AP site in DNA duplexes: thermodynamic characterization. Nucleic Acids Res. 37, 1411-1422 (2009). 
67. Sato, Y., Kageyama, T., Nishizawa, S. \& Teramae, N. Competitive binding of abasic site-binding ligands and masking ligands to DNA duplexes for the analysis of single-base mutation. Anal. Sci. 29, (2013).

68. Dai, Q. et al. Enhancement of the binding ability of a ligand for nucleobase recognition by introducing a methyl group. Anal. Sci. 22, 201-3 (2006).

69. Xiang, Y., Tong, A. \& Lu, Y. Abasic Site-Containing DNAzyme and Aptamer for Label-Free Fluorescent Detection of $\mathrm{Pb} 2+$ and Adenosine with High Sensitivity, Selectivity, and Tunable Dynamic Range. JACS 15352-15357 (2009).

70. Xiang, Y., Wang, Z., Xing, H., Wong, N. Y. \& Lu, Y. Label-Free Fluorescent Functional DNA Sensors Using Unmodified DNA : A Vacant Site Approach. Anal Chem 82, 4122-4129 (2010).

71. $\mathrm{Xu}, \mathrm{Z}$. et al. Label-free aptamer-based sensor using abasic site-containing DNA and a nucleobase-specific fluorescent ligand. Chem Commun 64456447 (2009).

72. Sato, Y., Honjo, A., Ishikawa, D., Nishizawa, S. \& Teramae, N. Fluorescent trimethyl-substituted naphthyridine as a ligand for $\mathrm{C}-\mathrm{C}$ mismatch detection in CCG trinucleotide repeats. Chem. Commun. (Camb) 47, 5885-5887 (2011).

73. Haskó, G., Linden, J., Cronstein, B. \& Pacher, P. Adenosine receptors: Therapeutic aspects for inflammatory and immune diseases. Nat. Rev. Drug Discov. 7, 759-770 (2008).

74. Faas, M. M., Sáez, T. \& de Vos, P. Extracellular ATP and adenosine: The Yin and Yang in immune responses? Mol. Aspects Med. 55, 9-19 (2017).

75. Chen, J. F., Eltzschig, H. K. \& Fredholm, B. B. Adenosine receptors as drug targets-what are the challenges? Nat. Rev. Drug Discov. 12, 265-286 (2013).

76. Fredholm, B. B. Adenosine, an endogenous distress signal, modulates tissue damage and repair. Cell Death Differ. 14, 1315-1323 (2007).

77. Hsu, W. Y. et al. Urinary nucleosides as biomarkers of breast, colon, lung, and gastric cancer in Taiwanese e81701. PLoS One 8, 8-15 (2013).

78. Ohta, A. et al. A2A adenosine receptor protects tumors from antitumor T cells. Proc. Natl. Acad. Sci. 103, 13132-13137 (2006). 
79. Sanghavi, B. J. et al. Real-time electrochemical monitoring of adenosine triphosphate in the picomolar to micromolar range using graphene-modified electrodes. Anal. Chem. 85, 8158-8165 (2013).

80. Marlinge, M. et al. Rapid Measurement of Adenosine Concentration in Human Blood Using Fixed Potential Amperometry: Comparison with Mass Spectrometry and High- Performance Liquid Chromatography. J. Anal. Bioanal. Tech. 08, 8-11 (2017).

81. Llaudet, E., Botting, N. P., Crayston, J. A. \& Dale, N. A three-enzyme microelectrode sensor for detecting purine release from central nervous system. Biosens. Bioelectron. 18, 43-52 (2003).

82. Ballarin, M., Fredholm, B. B., Ambrosio, S. \& Mahy, N. Extracellular levels of adenosine and its metabolites in the striatum of awake rats: Inhibition of uptake and metabolism. Acta Physiol. Scand. 142, 97-103 (1991).

83. Nutiu, R. \& Li, Y. Structure-switching signaling aptamers. J. Am. Chem. Soc. 125, 4771 (2003).

84. Stagg, J. \& Smyth, M. J. Extracellular adenosine triphosphate and adenosine in cancer. Oncogene 29, 5346-5358 (2010).

85. Beavis, P. A., Stagg, J., Darcy, P. K. \& Smyth, M. J. CD73: A potent suppressor of antitumor immune responses. Trends Immunol. 33, 231-237 (2012).

86. Barbu, M. \& Stojanovic, M. N. A Fresh Look at Adenosine-Binding DNA Motifs. ChemBioChem 13, 658-660 (2012).

87. White, R. J. \& Plaxco, K. W. Engineering new aptamer geometries for electrochemical aptamer-based sensors. IFMBE Proc. 7321, 732105 (2009).

88. Jarczewska, M., Górski, Ł. \& Malinowska, E. Electrochemical aptamerbased biosensors as potential tools for clinical diagnostics. Anal. Methods 8, 3861-3877 (2016).

89. Fan, C., Plaxco, K. W. \& Heeger, A. J. Electrochemical interrogation of conformational changes as a reagentless method for the sequence-specific detection of DNA. PNAS (2003).

90. Baker, B. R. et al. An Electronic, Aptamer-Based Small-Molecule Sensor for the rapid, Label-Free Detection of Cocaine in Adulterated samples and Biological Fluids. J. Am. Chem. Soc. 128, 3138-3139 (2006). 
91. Xiao, Y., Lubin, A. A., Heeger, A. J. \& Plaxco, K. W. Label-free electronic detection of thrombin in blood serum by using an aptamer-based sensor. Angew. Chemie - Int. Ed. 44, 5456-5459 (2005).

92. Uzawa, T., Cheng, R. R., White, R. J., Makarov, D. E. \& Plaxco, K. W. A mechanistic study of electron transfer from the distal termini of electrodebound, single-stranded DNAs. J. Am. Chem. Soc. 132, 16120-16126 (2010).

93. Swensen, J. S. et al. Continuous, real-time monitoring of cocaine in undiluted blood serum via a microfluidic, electrochemical aptamer-based sensor. J. Am. Chem. Soc. 131, 4262-4266 (2009).

94. Xiao, Y., Lai, R. Y. \& Plaxco, K. W. Preparation of electrode-immobilized, redox-modified oligonucleotides for electrochemical DNA and aptamerbased sensing. Nat. Protoc. 2, 2875-2880 (2007).

95. Levicky, R., Herne, T. M., Tarlov, M. J. \& Satija, S. K. Using self-assembly to control the structure of DNA monolayers on gold: A neutron reflectivity study. J. Am. Chem. Soc. 120, 9787-9792 (1998).

96. Herne, T. M. \& Tarlov, M. J. Characterization of DNA probes immobilized on gold surfaces. J. Am. Chem. Soc. 119, 8916-8920 (1997).

97. White, R. J., Phares, N., Lubin, A. A., Xiao, Y. \& Plaxco, K. W. Optimization of electrochemical aptamer-based sensors via optimization of probe packing density and surface chemistry. Langmuir 24, 10513-10518(2008).

98. Campuzano, S., Kuralay, F. \& Wang, J. Ternary Monolayer Interfaces for Ultrasensitive and Direct Bioelectronic Detection of Nucleic Acids in Complex Matrices. Electroanalysis 24, 483-493 (2012).

99. Fischer, N. O., Tok, J. B. \& Tarasow, T. M. Massively Parallel Interrogation of Aptamer Sequence, Structure and Function. PLoS One 3, 1-9 (2008).

100. Le, T. T., Chumphukam, O. \& Cass, A. E. G. Determination of minimal sequence for binding of an aptamer. A comparison of truncation and hybridization inhibition mehtods. RSC Adv. 4, 47227-47233 (2014).

101. Elle, I. C. et al. Selection of LNA-containing DNA aptamers againts recombinant human CD73. Mol. Biosyst. 11, 1260-1270 (2015).

102. Stojanovic, M. N., Prada, P. \& Landry, D. W. Aptamer-Based Folding Fluorescent Sensor for Cocaine . J. Am. Chem. Soc. 123,4928-4931 (2001). 
103. Lehman, I. R. \& Nussbaum, A. L. The deoxyribonucleases of Escherichia Coli. J. Biol. Chem. 239, 2628 (1964).

104. Zheng, D., Zou, R. \& Lou, X. Label-free fluorescent detection of ions, proteins, and small molecules using structure-switching aptamers, SYBR Gold, and Exonuclease I. Anal. Chem. 84, 3554-3560 (2012).

105. Demple, B. \& Harrison, L. Repair of oxidative damage to DNA: Enzymoogy and Biology. Annu. Rev. Biochem. 63, 915 (1994).

106. Wu, R. et al. Synchronous digestion of SV40 DNA by Exonuclease III. Biochemistry 15, 734 (1976).

107. Puapaiboon, U., Jai-nhuknan, J. \& Cowan, J. A. Characterization ofa multi-functional metal-mediated nuclease by MALDI-TOF mass spectrometry. Nucleic Acids Res. 29, 3652 (2001).

108. Hoheisel, J. D. On the activities of E. coli Exonuclease III. Anal. Biochem. 209, 238-246 (1993).

109. Metzger, W. \& Heumann, H. Methods in molecular biology. 148,

110. Wang, Z. et al. Introducing structure-switching functionality into smallmolecule-binding aptamers via nuclease-directed truncation. Nucleic Acids Res. 1-11 (2018). doi:10.1093/nar/gky305

111. Sayers, J. R. \& Eckstein, F. Properties of Overexpressed Phage T5 D15 Exonuclease. J. Biol. Chem. 265, 18311-18317 (1990).

112. Ceska, T. A., Sayers, J. R., Stier, G. \& Suck, D. A helical arch allowing single-stranded DNA to thread through T5 5'-exonuclease. Nature 382, 90 (1996).

113. Artymiuk, P. J., Ceska, T. A., Suck, D. \& Sayers, J. R. Prokaryotic 5 ' -3 ' exonucleases share a common core structure with gamma-delta resolvase. Nucleic Acids Res. 25, 4224-4229 (1997).

114. Garforth, S. J. et al. Unusually wide co-factor tolerance in a metalloenzyme ; divalent metal ions modulate endo - exonuclease activity in T5 exonuclease. Nucleic Acids Res. 29, 2772-2779 (2001).

115. Lehner, K. R. \& BBaumann, M. H. Psychoactive 'Bath Salts ': Compounds , Mechanisms, and Toxicities. Neuropsychopharmacol. Rev. 38, 242 (2013). 
116. Glennon, R. A. \& Young, R. Neurobiology of 3,4-

methylenedioxypyrovalerone (MDPV) and $\alpha$-pyrrolidinovalerophenone ( $\alpha$ PVP). Brain Res. Bull. 126, 111-126 (2016).

117. Aarde, S. M., Huang, P. K., Creehan, K. M., Dickerson, T. J. \& Taffe, M. A. The novel recreational drug 3,4-methylenedioxypyrovalerone (MDPV) is a potent psychomotor stimulant: self-administration and locomotor activity in rats. Neuropharmacology 71, 130-140 (2013).

118. Wyman, J. F. et al. Postmortem tissue distribution of MDPV following lethal intoxication by 'bath salts'. J. Anal. Toxicol. 37, 182-185 (2013).

119. Prosser, J. M. \& Nelson, L. S. The toxicology of bath salts: a review of synthetic cathinones. J. Med. Toxicol. 8, 33-42 (2012).

120. Roda, E. et al. Evaluation of Two Different Screening ELISA Assays for Synthetic Journal of Clinical Toxicology Evaluation of Two Different Screening ELISA Assays for Synthetic Cathinones (Mephedrone / Methcathinone and MDPV ) with LC-MS Method in Intoxicated Patients. J. Clin. Toxicol. 6, 3 (2016).

121. Patel, A., Malinovska, L., Saha, S., Wang, J. \& Alberti, S. ATP as a biological hydrotrope. Science 756, 753-756 (2017).

122. Morciano, G. et al. Use of luciferase probes to measure ATP in living cells and animals. Nat. Protoc. 12, 1542-1562 (2017).

123. Di Virgilio, F. \& Adinolfi, E. Extracellular purines, purinergic receptors and tumor growth. Oncogene 36, 293-303 (2017).

124. Pellegatti, P. et al. Increased Level of Extracellular ATP at Tumor Sites : In Vivo Imaging with Plasma Membrane Luciferase. PLoS One 3, 1-9 (2008).

125. Özalp, V. C., Nielsen, L. J. \& Olsen, L. F. An Aptamer-Based Nanobiosensor for Real-Time Measurements of ATP Dynamics. ChemBioChem 11, 2538-2541 (2010).

126. Di Chiara, G. \& Imperato, A. Drugs abused by humans preferentially increase synaptic dopamine concentrations in the mesolimbic system of freely moving rats. Proc. Natl. Acad. Sci. USA 85, 5274-5278 (1988).

127. Wise, R. A. et al. Fluctuations in nucleus accumbens dopamine concentration during intravenous cocaine self-administration in rats. Psychopharmacology (Berl). 120, 10-20 (1995). 
128. Ritz, M. C., Lamb, R. J., Goldberg, S. R. \& Kuhar, M. J. Cocaine receptors on dopamine transporters are related to self-administration of cocaine. Science 237, 1219-1223 (1987).

129. Kilty, J. E., Lorang, D. \& Amara, S. G. Cloning and expression of a cocaine-sensitive rat dopamine transporter. Science 254, 578-579 (1991).

130. Lange, R. A. \& Hillis, D. L. Cardiovascular complications of cocaine use . N. Engl. J. Med 345, 351-358 (2001).

131. Huang, X., Gu, H. H. \& Zhan, C. G. Mechanism for cocaine blocking the transport of dopamine: Insights from molecular modeling and dynamics simulations. J. Phys. Chem. B 113, 15057-15066 (2009).

132. Goeders, N. E. \& Smith, J. E. Cortical dopaminerginic involvement in cocaine reinforcement. Science 221, 773-775 (1983).

133. Mendelson, J. H. \& Mello, N. K. Management of cocaine abuse and dependence. N. Engl. J. Med 334, 965-972 (1996).

134. Marzuck, P. M. et al. Fatal Injuries after cocaine use as a leading cause of death among young adults in New York city. N. Engl. J. Med 332, 17531757 (1995).

135. Segura, J. et al. Immunological screening of drugs of abuse and gas chromatographic-mass spectrometric confirmation of opiates and cocaine in hair. J. Chromatogr. B 724, 9-21 (1999).

136. Schneider, R. S., Lindquist, P., Wong, E. T., Rubenstein, K. E. \& Ullman, E. F. Homogeneous enzyme immunoassay for oppiates in urine. Clin. Chem. 19, 821-825 (1973).

137. Melanson, S. E. The utility of immunoassays for urine drug testing. Clin. Lab. Med. 32, 429-447 (2012).

138. Brahm, N. C., Yeager, L. L., Fox, M. D., Farmer, K. C. \& Palmer, T. A. Commonly prescribed medications and potential false-positive urine drug screens . Am. J. Heal. Pharm. 67, 1344-1350 (2010).

139. Jayasena, S. D. Aptamers: An emerging class of molecules that rival antibodies in diagnostics. Clin. Chem. 45, 1628-1650 (1999).

140. Du, Y. et al. G-Quadruplex-based DNAzyme for colorimetric detection of cocaine: Using magnetic nanoparticles as the separation and amplification element. Analyst 136, 493-497 (2011). 
141. Zhang, J. et al. Visual cocaine detection with gold nanoparticles and rationally engineered aptamer structures. Small 4, 1196-1200 (2008).

142. Zhang, C. \& Johnson, L. W. Single Quantum-Dot-Based Aptameric Nanosensor for Cocaine. Anal. Chem. 81, 3051-3055 (2009).

143. Liu, J., Lee, J. H. \& Lu, Y. Quantum Dot Encoding of Aptamer-Linked Nanostructures for One-Pot Simultaneous Detection of Multiple Analytes. Anal Chem 79, 4120-25 (2007).

144. Baker, B. R. et al. An electronic, aptamer-based small-molecule sensor for the rapid, label-free detection of cocaine in adulterated samples and biological fluids. J. Am. Chem. Soc. 128, 3138-3139 (2006).

145. Cekan, P., Jonsson, E. O. \& Sigurdsson, S. T. Folding of the cocaine aptamer studied by EPR and fluorescence spectroscopies using the bifunctional spectroscopic probe C. Nucleic Acids Res. 37, 3990-3995 (2009).

146. Niu, S., Lou, X., Jiang, Y. \& Lin, J. A novel Fluorescence Sensor for Cocaine with Signal Amplification through cycling Exo-Cleaving with a hairpin probe. Anal. Lett. 45, 1919-1927 (2012).

147. He, J. L. et al. Fluorescence aptameric sensor for strand displacement amplification detection of cocaine. Anal. Chem. 82, 1358-1364(2010).

148. Qiu, L. et al. A novel label-free fluorescence aptamer-based sensor method for cocaine detection based on isothermal circuclar strand-displacement amplification and graphene oxide absoprtion . New J. Chem. 37, 39984003 (2013).

149. Neves, M. A., Reinstein, O., Saad, M. \& Johnson, P. E. Defining the secondary structural requirements of a cocaine-binding aptamer by a thermodynamic and mutation study. Biophys. Chem. 153, 9-16 (2010).

150. Kobori, A., Horie, S., Suda, H., Saito, I. \& Nakatani, K. The SPR sensor detecting cytosine-cytosine mismatches. J. Am. Chem. Soc. 126, 557-62 (2004).

151. Suda, H., Kobori, A., Zhang, J., Hayashi, G. \& Nakatani, K. N,N'-Bis(3aminopropyl)-2,7-diamino-1,8-naphthyridine stabilized a single pyrimidine bulge in duplex DNA. Bioorg. Med. Chem. 13, 4507-12 (2005).

152. Rajendar, B., Sato, Y., Nishizawa, S. \& Teramae, N. Improvement of base selectivity and binding affinity by controlling hydrogen bonding motifs between nucleobases and isoxanthopterin: application to the detection of T/C mutation. Bioorg. Med. Chem. Lett. 17, 3682-5 (2007). 
153. Zhao, G.-J. \& Han, K.-L. Ultrafast hydrogen bond strengthening of the photoexcited fluorenone in alcohols for facilitating the fluorescence quenching. J. Phys. Chem. A 111, 9218-23 (2007).

154. Huang, G.-J. et al. Site-selective hydrogen-bonding-induced fluorescence quenching of highly solvatofluorochromic GFP-like chromophores. Org. Lett. 14, 5034-7 (2012).

155. Jean, J. M. \& Hall, K. B. 2-Aminopurine fluorescence quenching and lifetimes: role of base stacking. Proc. Natl. Acad. Sci. U. S. A. 98, 37-41 (2001).

156. Hong, C., Hagihara, M. \& Nakatani, K. Ligand-assisted complex formation of two DNA hairpin loops. Angew. Chem. Int. Ed. Engl. 50, 4390-3(2011).

157. Zheng, D., Zou, R. \& Lou, X. Label-free fluorescent detection of ions, proteins, and small molecules using structure-switching aptamers, SYBR Gold, and exonuclease I. Anal. Chem. 84, 3554-60 (2012).

158. Toennes, S. W., Thiel, M., Walther, M. \& Kauert, G. F. Studies on metabolic pathways of cocaine and its metabolites using microsome preparations from rat organs. Chem . Res. Toxicol. 16, 375-381 (2003).

159. Schindler, C. W. \& Goldberg, S. R. Accelerating cocaine metabolism as an approach to treat cocaine abuse and toxicity . Futur. Med. Chem. 4, 163175 (2012).

160. Thongboonkerd, V., Chutipongtanate, S. \& Kanlaya, R. Systematic Evaluation of Sample Preparation Methods for Gel-Based Human Urinary Proteomics: Quantity, Quality, and Variability - Journal of Proteome Research (ACS Publications). J. Proteome Res. 5, 183-191 (2006).

161. Leiner, M. J. P., Hubmann, M. R. \& Wolfbeis, O. S. The total fluorescence of human urine. Anal. Chim. Acta 198, 13-23 (1987).

162. Kušnír, J., Dubayová, K., Lešková, L. \& Lajtár, M. Concentration Matrices-Solutions for Fluorescence Definition of Urine. Anal. Lett. 38, 1559-1567 (2005).

163. Dubayová, K., Kušnír, J. \& Podracká, L. Diagnostic monitoring of urine by means of synchronous fluorescence spectrum. J. Biochem. Biophys. Methods 55, 111-119 (2003).

164. Krebs, H. a. Chemical composition of blood plasma and serum. Annu. Rev. Biochem. 19, 409-430 (1950). 
165. Cheaib, Z. \& Lussi, A. Role of amylase, mucin, IgA and albumin on salivary protein buffering capacity: A pilot study. J. Biosci. 38, 259-265 (2013).

166. Sigma-Aldrich. Cocaine/Benzoylecgonine Direct ELISA.

167. Linden, J. Role of adenosine in response to vascular inflammation. Arterioscler. Thromb. Vasc. Biol. 32, 843-844 (2012).

168. Xu, Y. et al. Regulation of endothelial intracellular adenosine via adenosine kinase epigenetically modulates vascular inflammation. Nat. Commun. 8, (2017).

169. Schulte, G. \& Fredholm, B. B. Signalling from adenosine receptors to mitogen-activated protein kinases. Cell. Signal. 15, 813-827 (2003).

170. Longhi, M. S., Robson, S. C., Bernstein, S. H., Serra, S. \& Deaglio, S. Biological functions of ecto-enzymes in regulating extracellular adenosine levels in neoplastic and inflammatory disease states. J. Mol. Med. 91, 165172 (2013).

171. Taniai, H. et al. A Simple Quantitative Assay for Urinary Adenosine Using Column-Switching High-Performance Liquid Chromatography. Tohoku J. Exp. Med. 208, 57-63 (2006).

172. Vijayamahantesh et al. Adenosine generated by ectonucleotidases modulates the host immune system during visceral leishmaniasis. Cytokine 91, 170-179 (2017).

173. Boison, D. Adenosine Kinase: Exploitation for Therapeutic Gain. Pharmacol. Rev. 65, 906-943 (2013).

174. Corciulo, C. et al. Endogenous adenosine maintains cartilage homeostasis and exogenous adenosine inhibits osteoarthritis progression. Nat. Commun. 8, 15019 (2017).

175. Van Dycke, A. et al. Quantitative analysis of adenosine using liquid chromatography/atmospheric pressure chemical ionization-tandem mass spectrometry (LC/APCI-MS/MS). J. Chromatogr. B Anal. Technol. Biomed. Life Sci. 878, 1493-1498 (2010).

176. Roncancio, D. et al. A Label-Free Aptamer-Fluorophore Assembly for Rapid and Speci fi c Detection of Cocaine in Bio fl uids. Anal. Chem. 86, 11100-11106 (2014). 
177. Ahn, J. K., Kim, H. Y., Baek, S. \& Park, H. G. A new sadenosylhomocysteine hydrolase-linked method for adenosine detection based on DNA-templated fluorescent Cu/Ag nanoclusters. Biosens. Bioelectron. 93, 330-334 (2017).

178. BioVision. Adenosine Assay Kit ( Fluorometric).

179. Palmer, C. \& Vannucci, R. C. Thine in Relation To Tissue Nucleotides and Purines in Rat Striatum During Transient Ischemia. (1997).

180. Llaudet, E., Hatz, S., Droniou, M. \& Dale, N. Microelectrode biosensor for real-time measurement of ATP in biological tissue. Anal. Chem. 77, 32673273 (2005).

181. Lubin, A. A., Lai, R. Y., Baker, B. R., Heeger, A. J. \& Plaxco, K. W. Sequence-specific, electronic detection of oligonucleotides in blood, soil, and foodstuffs with the reagentless, reusable E-DNA sensor. Anal. Chem. 78, 5671-5677 (2006).

182. Liu, J., Wagan, S., Dávila Morris, M., Taylor, J. \& White, R. J. Achieving reproducible performance of electrochemical, folding aptamer-based sensors on microelectrodes: Challenges and prospects. Anal. Chem. 86, 11417-11424 (2014).

183. White, R. et al. Generation of species cross-reactive aptamers using 'toggle' SELEX. Mol. Ther. 4, 567-574 (2001).

184. Tokunaga, T. et al. Cell surface-anchored fluorescent aptamer sensor enables imaging of chemical transmitter dynamics. J. Am. Chem. Soc.134, 9561-9564 (2012).

185. Nielsen, L. J., Olsen, L. F. \& Ozalp, V. C. Aptamers embedded in polyacrylamide nanoparticles: A tool for in vivo metabolite sensing. ACS Nano 4, 4361-4370 (2010).

186. Entzian, C. \& Schubert, T. Studying small molecule-aptamer interactions using MicroScale Thermophoresis (MST). Methods 97, 27-34 (2016).

187. Deng, Q., German, I., Buchanan, D. \& Kennedy, R. T. Retention and separation of adenosine and analogues by affinity chromatography with an aptamer stationary phase. Anal. Chem. 73, 5415-5421 (2001).

188. Li, X., Ding, X. \& Fan, J. Nicking endonuclease-assisted signal amplification of a split molecular aptamer beacon for biomolecule detection using graphene oxide as a sensing platform. Analyst 140, 7918-7925 (2015). 
189. Huang, P. J. J. \& Liu, J. Flow cytometry-assisted detection of adenosine in serum with an immobilized aptamer sensor. Anal. Chem. 82, 4020-4026 (2010).

190. Vaish, N. K., Larralde, R., Fraley, A. W., Szostak, J. W. \& McLaughlin, L. W. A novel, modification-dependent ATP-binding aptamer selected from an RNA library incorporating a cationic functionality. Biochemistry 42, 88428851 (2003).

191. Sazani, P. L., Larralde, R. \& Szostak, J. W. A small aptamer with strong and specific recognition of the triphosphate of ATP. J. Am. Chem. Soc. 126, 8370-8371 (2004).

192. Ricci, F., Lai, R. Y. \& Plaxco, K. W. Linear, redox modified DNA probes as electrochemical DNA sensors. Chem. Commun. 3768 (2007).

193. Lubin, A. A. \& Plaxco, K. W. Folding-based electrochemical biosensors: The case for responsive nucleic acid architectures. Acc. Chem. Res. 43, 496-505 (2010).

194. Plaxco, K. W. \& Soh, H. T. Switch-based biosensors: A new approach towards real-time, in vivo molecular detection. Trends Biotechnol. 29, 1-5 (2011).

195. Watkins, H. M., Vallée-Bélisle, A., Ricci, F., Makarov, D. E. \& Plaxco, K. W. Entropic and electrostatic effects on the folding free energy of a surfaceattached biomolecule: An experimental and theoretical study. J. Am. Chem. Soc. 134, 2120-2126 (2012).

196. Zuo, X. et al. A target-responsive electrochemical aptamer switch (TREAS) for reagentless detection of nanomolar ATP. J. Am. Chem. Soc. 129, 1042-1043 (2007).

197. Zhang, Z., Oni, O. \& Liu, J. New insights into a classic aptamer: Binding sites, cooperativity and more sensitive adenosine detection. Nucleic Acids Res. 45, 7593-7601 (2017).

198. Arnaut, V., Langecker, M. \& Simmel, F. C. Nanopore force spectroscopy of aptamer-ligand complexes. Biophys. J. 105, 1199-1207 (2013).

199. Fisica, C. \& Milano, U. Real surface area measurements in electrochemistry. Pure Appl. Chem. 63, 711-734 (1991).

200. Steel, A. B., Herne, T. M. \& Tarlov, M. J. Electrochemical quantitation of DNA immobilized on gold. Anal. Chem. 70, 4670-4677 (1998). 
201. Wang, H. et al. Instrument-free quantitative gold nanoparticle-based liquidphase colorimetric assays for use in resource-poor environments. Chem. Commun. 53, 8407-8410 (2017).

202. Chovelon, B. et al. ELAKCA: Enzyme-Linked Aptamer Kissing Complex Assay as a Small Molecule Sensing Platform. Anal. Chem. 88, 2570-2575 (2016).

203. Xu, X., Wei, H. \& Jiang, W. A target triggered proximity combination-based fluorescence sensing strategy for adenosine detection. Analyst 142, 22472252 (2017).

204. Baaske, P., Wienken, C. J., Reineck, P., Duhr, S. \& Braun, D. Optical thermophoresis for quantifying the buffer dependence of aptamer binding. Angew. Chemie - Int. Ed. 49, 2238-2241 (2010).

205. Chase, F. Effects of Adenosine Triphosphate the Fumarase Reaction * and Magnesium lons on. 244, (1969).

206. Pecoraro, V. L., Hermes, J. D. \& Cleland, W. W. Stability Constants of $\mathrm{Mg} 2+$ and $\mathrm{Cd} 2+$ Complexes of Adenine Nucleotides and Thionucleotides and Rate Constants for Formation and Dissociation of MgATP and MgADP. Biochemistry 23, 5262-5271 (1984).

207. Huang, F., Hao, G., Wu, F. \& Feng, G. Fluorescence sensing of ADPover ATP and PPi in 100\% aqueous solution. Analyst 140, 5873-5876 (2015).

208. Lin, C. H. \& Patel, D. J. Structural basis of DNA folding and recognition in an AMP-DNA aptamer complex: Distinct architectures but common recognition motifs for DNA and RNA aptamers complexed to AMP. Chem. Biol. 4, 817-832 (1997).

209. Pang, J., Zhang, Z. \& Jin, H. Effect of structure variation of the aptamerDNA duplex probe on the performance of displacement-based electrochemical aptamer sensors. Biosens. Bioelectron. 77, 174-181 (2016).

210. Xia, T., Yuan, J. \& Fang, X. Conformational Dynamics of an ATP-binding DNA Aptamer : A Single-Molecule Study Conformational Dynamics of an ATP-binding DNA Aptamer: A Single-Molecule Study. (2013).

211. Sachan, A., Ilgu, M., Kempema, A., Kraus, G. A. \& Nilsen-Hamilton, M. Specificity and Ligand Affinities of the Cocaine Aptamer: Impact of Structural Features and Physiological NaCl. Anal. Chem. 88, 7715-7723 (2016). 
212. Kypr, J., Kejnovská, I., Renčiuk, D. \& Vorlíčková, M. Circular dichroism and conformational polymorphism of DNA. Nucleic Acids Res. 37, 1713-1725 (2009).

213. Alberty, R. A., Smith, R. M. \& Bock, R. M. The apparent ionization constants of the adenosinephosphates and related compounds. (1950).

214. Ricci, F., Lai, R. Y., Heeger, A. J., Plaxco, K. W. \& Sumner, J. J. Effect of molecular crowding on the response of an electrochemical DNA sensor. Langmuir 23, 6827-6834 (2007).

215. Kang, D., Ricci, F., White, R. J. \& Plaxco, K. W. Survey of Redox-Active Moieties for Application in Multiplexed Electrochemical Biosensors. Anal. Chem. 88, 10452-10458 (2016).

216. Wu, J., Campuzano, S., Halford, C., Haake, D. A. \& Wang, J. Ternary Surface Mono layers for Ultrasensitive (Zeptomole) Amperometric Detection of Nucleic Acid Hybridization without Signal Amplification. Anal. Chem. 82, 8830-8837 (2010).

217. Oh, S. S., Plakos, K., Lou, X., Xiao, Y. \& Soh, H. T. In vitro selection of structure-switching, self-reporting aptamers. Proc. Natl. Acad. Sci. U. S. A. 107, 14053-8 (2010).

218. SantaLucia, J. \& Hicks, D. The Thermodynamics of DNA Structural Motifs. Annu. Rev. Biophys. Biomol. Struct. 33, 415-440 (2004).

219. Garforth, S. J., Ceska, T. A., Suck, D. \& Sayers, J. R. Mutagenesis of conserved lysine residues in bacteriophage T5 5'-3' exonuclease suggests separate mechanisms of endo-and exonucleolytic cleavage. Proc. Natl. Acad. Sci. U. S. A. 96, 38-43 (1999).

220. Sayers, J. R., Evans, D. \& Thomson, J. B. Identification and eradication of a denatured DNA isolated during alkaline lysis-based plasmid purification procedures. Anal. Biochem. 241, 186-189 (1996).

221. Sayers, J. R. \& Eckstein, F. A single-strand specific endonuclease activity copurifies with overexpressed T5 D15 exonuclease. Nucleic Acids Res. 19, 4127-4132 (1991).

222. Zuker, M. Mfold web server for nucleic acid folding and hybridization prediction. Nucleic Acids Res. 31, 3406-3415 (2003).

223. Wang, M. et al. One-step, ultrasensitive, and electrochemical assay of microRNAs based on 77 exonuclease assisted cyclic enzymatic amplification. Anal. Chem. 86, 5606-5610 (2014). 
224. Cui, L. et al. A T7 exonuclease-assisted cyclic enzymatic amplification method coupled with rolling circle amplification: a dual-amplification strategy for sensitive and selective microRNA detection. Chem. Commun. 50, 1576-1578 (2014).

225. Kerr, C. \& Sadowski, P. Gene 6 Exonuclease of Bacteriophage T7. J. Biol. Chem. 247, 311 (1971).

226. $\mathrm{Wu}, \mathrm{Z} . \mathrm{K}$. et al. Single-base mismatch discrimination by $\mathrm{T7}$ exonuclease with target cyclic amplification detection. Chem. Commun. 51, 2954-2956 (2015).

227. Hughes, Z. E. \& Walsh, T. R. Structural Disruption of an AdenosineBinding DNA Aptamer on Graphene: Implications for Aptasensor Design. ACS Sensors 2, 1602-1611 (2017).

228. Serrano-Santos, M. B., Llobet, E., Özalp, V. C. \& Schäfer, T. Characterization of structural changes in aptamer films for controlled release nanodevices. Chem. Commun. 48, 10087 (2012).

229. Yang, K.-A., Pei, R., Stefanovic, D. \& Stojanovic, M. N. Optimizing crossreactivity with evolutionary search for sensors. J. Am. Chem. Soc. 134, 1642-7 (2012).

230. Yang, K. A. et al. High-Affinity Nucleic-Acid-Based Receptors for Steroids. ACS Chem. Biol. 12, 3103-3112 (2017).

231. Kempfle, M., Muller, R., Palluk, R. \& Zachariasse, K. A. Fluorescence of 3keto-steroids in aqueous solution. Eur. Biophys. J. 14, 29 (1986).

232. Garforth, S. J. \& Sayers, J. R. Structure-specific DNA binding by bacteriophage T5 5' $\rightarrow$ 3' exonuclease. Nucleic Acids Res. 25, 3801-3807 (1997).

233. Hampshire, A. J., Rusling, D. A., Broughton-head, V. J. \& Fox, K. R. Footprinting: A method for determining the sequence selectivity, affinity and kinetics of DNA-binding ligands. Methods 42, 128-140 (2007).

234. Yu, H., Canoura, J., Guntupalli, B., Lou, X. \& Xiao, Y. A cooperativebinding spli aptamer assay for rapid, specific and ultra-sensitive fluorescence detection of cocaine in saliva. Chem. Sci. 8, 131-141(2016).

235. Sato, Y. et al. Abasic site-binding ligands conjugated with cyanine dyes for 'off-on' fluorescence sensing of orphan nucleobases in DNA duplexes and DNA-RNA hybrids. Chem Commun 50, 18-21 (2014). 
VITA

\title{
DANIEL RONCANCIO
}

\author{
2008-2013 \\ B.S., Chemistry \\ Florida International University \\ Miami, FL \\ 2013-2015 \\ McNair Graduate fellow \\ Florida International University \\ Miami, FL \\ 2013-2015 \\ M.S., Chemistry \\ Florida International University \\ Miami, FL \\ 2015-2018 \\ Teaching assistant \\ Florida International University \\ Miami, FL \\ 2018 \\ Dissertation year fellowship \\ Florida International University \\ Miami, FL \\ 2013-2018 \\ Doctoral candidate \\ Florida International University \\ Miami, FL
}

\section{PUBLICATIONS AND PRESENTATIONS}

1. Roncancio D., Canoura J., Xiao Y. (2018) Engineering a specific aptamer for electrochemical detection of Adenosine in whole serum. In preparation

2. Roncancio D., Xiao Y. (2018) Nuclease truncation of aptamers for electrochemical sensors. In preparation

3. Roncancio D., Canoura J., Xiao Y. (2018) Engineering a specific aptamer for electrochemical detection of Adenosine in whole serum. Oral presentation, Conference of Undergraduate Research at FIU (CURFIU), Miami, FL, March 28, Miami, FL.

4. Roncancio D., Canoura J., Xiao Y. (2018) Engineering a specific aptamer for electrochemical detection of Adenosine in whole serum. Oral presentation, FIU's annual Scholarly Forum during Graduate Student 
Appreciation Week (GSAW), Florida International University, Miami, FL, March 21.

5. Roncancio D., Yu H.X., Xu X.W. \& Xiao Y. (2017) A label-free aptamerfluorophore assembly for highly sensitive and specific detection of cocaine. Oral presentation, Pittcon 2017, Chicago, Illinois, March 5th.

6. Yu H.X., Xu X.W., Liang P.P., Loh K.Y., Guntupalli B., Roncancio D. \& Xiao Y. * (2017) A broadly-applicable assay for rapidly and accurately quantifying DNA surface coverage on diverse particles. Bioconjugate Chem., 28, 933 943.

7. Canoura J., Wang Z.W., Yu H.X., Ng B., Roncancio D. \& Xiao Y. (2016) Utilizing nuclease screening of ligand-aptamer complexes to enhance specificity of an aptamer-based cocaine assay. Oral presentation, 2016 Conference of Undergraduate Research at FIU (CURFIU), Miami, FL, March $30-31$.

8. Roncancio D., Yu H.X., Xu X.W., Wu S., Liu R., Debord J., Lou X.H. \& Xiao Y. (2016) A label-free aptamer-fluorophore assembly for highly sensitive and specific detection of cocaine. Oral presentation, FIU's annual Forum for Graduate Student Appreciation (GSAW), Florida International University, Miami, FL, March, 28.

9. Roncancio D., Yu H.X., Xu X.W., Wu S., Liu R., Debord J., Lou X.H. \& Xiao Y. (2016) A label-free aptamer-fluorophore assembly for highly sensitive and specific detection of cocaine. Oral presentation, Fifth Annual Forensic Science Symposium, Miami, FL, March 15 - 16.

10. Wang Z.W., Yu H.X., Ng B., Roncancio D., Canoura J. \& Xiao Y. (2015) Amplified colorimetric detection of cocaine in oral fluid based on exonuclease-assisted aptamer strand recycling. Poster presentation, SOFT 2015, Atlanta, Georgia, October 19 - 23.

11. Roncancio D., Wu S., Yu H.X., Xu X.W., Liu R., Debord J., Lou X.H. \& Xiao Y. (2014) A label-free aptamer-fluorophore assembly for highly sensitive and specific detection of cocaine. Poster presentation, 2014 MRS Fall Meeting \& Exhibit, Boston, Massachusetts, November 30 - December 5.

12. Roncancio D., Yu H.X., Xu X.W., Wu S., Liu R., Debord J., Lou X.H. \& Xiao Y. * (2014) A label-free aptamer-fluorophore assembly for rapid and specific detection of cocaine in biofluids. Anal. Chem., 86, $1100-11106$. 